\title{
Photodissoziation von Halogenwasserstoff- und orientierten \\ Wasserstoff-Edelgas-Halogen-Molekülen in Clusterumgebungen
}

\author{
Dissertation \\ zur Erlangung des Doktorgrades \\ der Mathematisch-Naturwissenschaftlichen Fakultäten \\ der Georg-August-Universität zu Göttingen \\ vorgelegt von \\ Nils Hendrik Nahler \\ aus \\ Kassel
}

Göttingen 2002 
D7

Referent: Prof. Dr. U. Buck

Koreferent: Prof. Dr. W. Lauterborn

Tag der mündlichen Prüfung: 28.10.2002 
Discunt, ut scis, Stoici nostri duo esse in rerum natura ex quibus omnia fiant, causam et materiam. Materia iacet iners, res ad omnia parata, cessatura si nemo moveat; causa autem, id est ratio, materiam format et quocumque vult versat, ex illa varia opera producit. Esse ergo debet unde fiat aliquid, deinde a quo fiat: hoc causa est, illud materia.

Seneca, Epistulae morales ad Lucilium, Liber VII, Epistula LXV, 2

Unsere Stoiker sagen, wie du weißt, in der Natur der Dinge gebe es zweierlei, aus dem alles entstehe, Ursache und Materie. Die Materie liegt untätig da, eine Sache, die zu allem bereit ist, die, falls sie niemand in Bewegung setzt, immer träge ist. Die Ursache dagegen, das heißt die Vernunft, gestaltet die Materie und wendet sie, wohin sie will und bringt aus ihr verschiedene Werke hervor. Also muß es etwas geben, woraus etwas entsteht, sodann etwas, wodurch es entsteht: letzteres ist die Ursache, ersteres die Materie. 



\section{Übersicht}

In dieser Arbeit wird die Photodissoziation von Molekülen in Clusterumgebungen behandelt. Der Schwerpunkt liegt hierbei auf der Wechselwirkung der Dissoziationsprodukte mit den Käfigatomen. Die Edelgas-Cluster bekannter Größe werden mit den unterschiedlichen Halogenwasserstoffmolekülen $\mathrm{HCl}, \mathrm{HBr}$ oder $\mathrm{HI}$ dotiert. Die Moleküle befinden sich je nach Präparationsmethode auf der Oberfläche oder im Inneren des Clusters. Die Auswahl dieser Systeme ist in ihrer einfacheren theoretischen Behandlung im Vergleich zum Festkörper begründet. Dies bedingt, daß ein qualitativer und quantitativer Vergleich mit Rechnungen möglich ist. Die Moleküle werden im UV Bereich bei Wellenlängen von 243 und $193 \mathrm{~nm}$ dissoziiert. Die dabei frei werdenden Wasserstoffatome werden in einem 2+1 REMPI Prozeß ionisiert, wobei ebenfalls Photonen einer Wellenlänge von $243 \mathrm{~nm}$ zur Anwendung kommen. Die entscheidende Meßgröße dieser Experimente ist die kinetische Energieverteilung der Wasserstofffragmente, die in einem Wiley-McLaren Flugzeitmassenspektrometer bestimmt wird. Das Verhältnis zwischen völlig abgebremsten und direkt austretenden Wassterstofffragmenten hängt vom Aufenthaltsort des Moleküls im Cluster, der genauen Position in der Oberfläche, dem Edelgas und für eingebettete Moleküle von der Clustergröße ab. Ein zweites Thema dieser Arbeit ist der Nachweis von $\mathrm{HXel}$ und $\mathrm{HXeCl}$ Molekülen als Beispiele der ionisch gebundenen Wasserstoff-Edelgas-Halogen ( $\mathrm{HRgX})$ Komlexe. Sie werden im Photodissoziationsprozeß von $\mathrm{HI}$ und $\mathrm{HCl}$ an der Oberfläche von Xe Clustern gebildet. Diese Moleküle werden in einer Kombination aus starkem Laserfeld und schwachem elektrischen Feld orientiert. Der Nachweis ist über die anschließende Photodissoziation möglich, die zu einer asymmetrischen Verteilung im Flugzeitspektrum der Wasserstofffragmente führt. 


\section{Abstract}

The object of this experimental work is the photodissociation of molecules and the interaction of the products with the surrounding cage atoms. Rare gas clusters of known size distribution are generated and doped with the different hydrogen halide molecules $\mathrm{HCl}, \mathrm{HBr}$ and $\mathrm{HI}$. They are placed on the surface or in the interior of the cluster. These systems are chosen because of their easier theoretical handling in contrast to bulk matter and because of the possibility of direct comparison with calculations. The molecules are photodissociated in the UV region at 243 and $193 \mathrm{~nm}$. The outgoing $\mathrm{H}$ atoms are ionised in a $2+1 \mathrm{REMPI}$ scheme also at $243 \mathrm{~nm}$ and their kinetic energy is measured in a Wiley-McLaren timeof-flight mass spectrometer. The ratio between caged and unperturbed exiting $\mathrm{H}$ atoms strongly depends on the site, the special surface state, the cage material, and, for the embedded case, on the cluster size. As a second topic measurements are presented which demonstrate the generation of the molecules $\mathrm{HXel}$ and $\mathrm{HXeCl}$ as species of the ionic bound hydrogen-rare gas-halogen $(\mathrm{HRgX})$ complexes. They are generated in the photodissociation of $\mathrm{HI}$ and $\mathrm{HCl}$ molecules on Xe clusters. The detection is achieved by asymmetric time-offlight distributions caused by $\mathrm{H}$ atoms resulting from the photodissociation of the formed $\mathrm{HRgX}$ molecules oriented in combined strong laser and weak electrostatic fields. 


\section{Inhaltsverzeichnis}

$\begin{array}{ll}\text { Einleitung } & 1\end{array}$

1 Grundlagen 5

1.1 Photodissoziation . . . . . . . . . . . . . . . 5

1.1.1 Photodissoziation zweiatomiger Moleküle . . . . . . . . . 8

1.1 .2 Energiebilanz . . . . . . . . . . . . . . . . . . 8

1.1 .3 Winkelverteilung . . . . . . . . . . . . . . . . . . . 9

1.1.4 Photodissoziation von Halogenwasserstoffen . . . . . . . . . . . 10

1.1 .5 Käfigeffekt . . . . . . . . . . . . . . . . . . . 18

1.2 Clustererzeugung . . . . . . . . . . . . . . . . . . . 22

1.2.1 Düsenstrahlexpansion . . . . . . . . . . . . . . . 22

1.2.2 Clusterbildung und Größenverteilung . . . . . . . . . . . . . 24

1.2 .3 Erzeugung von Mischclustern . . . . . . . . . . . . . . . . . 27

1.2.4 Ikosaederstruktur von Edelgasclustern . . . . . . . . . . . . . 29

1.3 Flugzeit-Spektroskopie . . . . . . . . . . . . . . . . . . . . . . . . 30

1.3.1 Wiley-McLaren Flugzeitmassenspektrometer . . . . . . . . . 30

1.3.2 REMPI . . . . . . . . . . . . . . . . . . . . . . . . . . . . . 34

1.4 Eine neue Klasse von Molekülen: Wasserstoff-Edelgas-Halogen (HRgX) 37

1.4.1 Bildung und Eigenschaften . . . . . . . . . . . . . . . . 39

1.4.2 Orientierung von Molekülen in kombinierten elektrischen Wechselund statischen Feldern . . . . . . . . . . . . . . . . . 41

2 Experimenteller Aufbau $\quad 47$

2.1 Molekularstrahlapparatur . . . . . . . . . . . . . . . . . . . . . . 49

2.1.1 Quelle zur Erzeugung großer Ne Cluster . . . . . . . . . . . . . 51

2.1 .2 Clusterpräparation . . . . . . . . . . . . . . . . . . 53 
2.1.3 Strahlanalyse mittels Quadrupolmassenspektrometer . . . . . . 54

2.2 Lasersysteme . . . . . . . . . . . . . . . . . . . . . . . . . . . . 55

2.2.1 Lasersystem zur Erzeugung von $243 \mathrm{~nm} \ldots \ldots \ldots$

2.2.2 $\mathrm{ArF} / \mathrm{F}_{2}$-Excimer-Laser . . . . . . . . . . . . . . 58

2.2.3 Polarisierung und Einkopplung der Laserstrahlen . . . . . . . . . . 60

2.3 Wiley-McLaren Flugzeitmassenspektrometer (WMTOFMS) . . . . . . . . 63

2.3.1 WMTOFMS mit kurzer Driftstrecke . . . . . . . . . . . 63

2.3.2 WMTOFMS mit geschaltetem Abzugsfeld . . . . . . . . . 65

2.3.3 WMTOFMS mit langer abgeschirmter Driftstrecke . . . . . . . . 68

2.3.4 Untergrund-Massenspektrum im Hochfeldmodus . . . . . . . . 71

3 Auswertung und Simulation $\quad 73$

3.1 Analyse der gemessenen Flugzeitspektren . . . . . . . . . . . . . . . . . . . . . . . . . . 73

3.1 .1 Meßprinzip . . . . . . . . . . . . . . . . . . . . . . . 73

3.1 .2 Ermittlung der Signalspektren . . . . . . . . . . . 76

3.2 Transformation der Flugzeitspektren in Energieverteilungen . . . . . . . . 78

3.2.1 Monte-Carlo Simulation der Trajektorien im WM-TOFMS . . . . 80

3.2.2 Transformation poly-energetischer Flugzeitspektren . . . . . . . 85

4 Experimentelle Ergebnisse $\quad \mathbf{8 7}$

4.1 Photodissoziation von HI Molekülen auf Ar Clustern . . . . . . . . . . . . 88

4.2 Photodissoziation von $\mathrm{HBr}$ Molekülen in unterschiedlichen Clusterumgebungen . . . . . . . . . . . . . . . . . . . . . 92

4.2.1 Temperaturabhängigkeit der Energieverteilungen aus der Photodissoziation von $\mathrm{HBr}$ Molekülen auf $\mathrm{Ar}$ Clustern konstanter Größe . .

4.2.2 Photodissoziation von $\mathrm{HBr}$ Molekülen in Ar Clustern: Käfigeffekt in Abhängigkeit von der Clustergröße . . . . . . . . . . . . . 96

4.2.3 Photodissoziation von $\mathrm{HBr}$ Molekülen auf Ne Clustern . . . . . . 98

4.3 Photodissoziation von $\mathrm{HCl}$ Molekülen in unterschiedlichen Clusterumgebungen . . . . . . . . . . . . . . . . . . . 100

4.3.1 Photodissoziation von $\mathrm{HCl}$ Molekülen auf $\mathrm{Ne}, \mathrm{Ar}, \mathrm{Kr}$, und $\mathrm{Xe}$ Clustern102

4.3.2 Photodissoziation von $\mathrm{HCl}$ Molekülen auf Ne und Ar Clustern: GröBenabhängigkeit . . . . . . . . . . . . . . . . . 104

4.3.3 Photodissoziation von $\mathrm{HCl}$ Clustern und $(\mathrm{HCl})_{x} \mathrm{Ar}_{n}$ Mischclustern 107 
4.4 Bildung, Orientierung und Dissoziation von $\mathrm{HXel}$ und $\mathrm{HXeCl}$ Molekülen . 111

4.4.1 Energieverteilung der Dissoziation von $\mathrm{HXel}$ in Abhängigkeit vom Polarisationswinkel $\alpha$ und von der Feldrichtung im WMTOFMS . 111

4.4.2 Energieverteilung der Dissoziation von $\mathrm{HXeCl}$ in Abhängigkeit vom Polarisationswinkel $\alpha \ldots \ldots \ldots \ldots \ldots$

5 Diskussion $\quad 121$

5.1 Position der HX Moleküle in der Edelgasclusterumgebung . . . . . . . . 121

5.1.1 $\mathrm{HBr}$ Molekül in der Ar Clusteroberfläche . . . . . . . . . . . . . . 125

5.1.2 HI Molekül in der Ar Clusteroberfläche . . . . . . . . . . . . . . . 127

5.1.3 $\mathrm{HBr}$ und $\mathrm{HCl}$ Moleküle in flüssigen Ne Clustern . . . . . . . . . . 127

5.1.4 $\mathrm{HBr}$ Molekül im Zentrum des Ar Clusters . . . . . . . . . . . . . 129

5.1.5 $\mathrm{HCl}$ Molekül in der Oberfläche von Edelgasclustern . . . . . . . . 132

5.1.6 $\mathrm{HCl}$ Molekül in $\mathrm{Ar}$ und $\mathrm{HCl}$ Clustern . . . . . . . . . . . . . . . 135

5.1 .7 Zusammenfassung . . . . . . . . . . . . . . . . . . . . 137

5.2 Winkelverteilung in den Produktkanälen . . . . . . . . . . . . 137

$5.2 .1 \quad \mathrm{HI}$ : Laserpolarisation $\alpha \ldots \ldots \ldots$

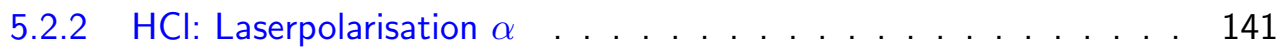

5.2 .3 Zusammenfassung . . . . . . . . . . . . . . . . . . . . . . . . 142

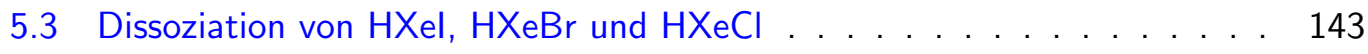

5.3 .1 Bildung . . . . . . . . . . . . . . . . . . . . . 143

5.3.2 Orientierung und Laserpolarisation . . . . . . . . . . . . . . . 145

5.3.3 Potentiale, Schwingungen und Dissoziation . . . . . . . . . . . 148

$\begin{array}{ll}\text { Zusammenfassung und Ausblick } & 151\end{array}$

$\begin{array}{ll}\text { Abbildungsverzeichnis } & 155\end{array}$

$\begin{array}{lr}\text { Tabellenverzeichnis } & 159\end{array}$

$\begin{array}{ll}\text { Literaturverzeichnis } & 161\end{array}$ 



\section{Einleitung}

Die Photodissoziation als lichtinduzierter Zerfall eines Moleküls spielt eine wichtige Rolle in atmosphärischen Reaktionen. Zentrale Bedeutung in der Atmosphärenchemie besitzen die Halogenwasserstoffe $\mathrm{HCl}$ und $\mathrm{HBr}$ sowie weitere chlorhaltige Verbindungen wie $\mathrm{HOCl}$, $\mathrm{OCIO}$ und $\mathrm{ClONO}_{2}$. Unter Bestrahlung mit Sonnenlicht setzen diese Moleküle Chlor- und Brom-Atome frei, die direkt in einem katalytischen Zyklus Ozon abbauen [Row91, Lev85].

Informationen über den Ablauf des Photodissoziationsprozesses lassen sich zum einen direkt aus zeitaufgelöste Messungen [Zew93] gewinnen, zum anderen aus Untersuchungen, die sich auf die asymptotischen Eigenschaften der Dissoziationsprodukte beziehen (Bevölkerung der Produktkanäle, kinetische Energie der Produkte) [Sat01, Ash96, Sch93]. Die Photodissoziation der diatomaren Halogenwasserstoffmoleküle führt in nur zwei Produktkanäle, und im Vergleich zu polyatomaren Molekülen sind bei innen nur eine geringe Anzahl von Zuständen beteiligt. Nicht-adiabatische Kopplungen zwischen diesen Zuständen bewirken eine Umverteilung in der Population der Produktkanäle [Peo97, Ale93].

In Studien der chemischen Reaktionsdynamik nimmt die Photodissoziation in chemisch inerten Medien eine zentrale Position ein. Zahlreiche Untersuchungen sind dazu in Hochdruckgasen [Sch87], Flüssigkeiten [Har88] und festen Matrizen [Apk99] durchgeführt worden. Im Mittelpunkt steht dabei der Einfluß des umgebenden Lösungsmittels oder Käfigs auf die Dissoziationsdynamik. Wechselwirkungen der Dissoziationsfragmente mit der Umgebung können zu Reaktionen wie Rekombination oder Bildung neuer Moleküle führen (Käfigeffekt). Eine weitere mögliche Umgebung zur Untersuchung dieser Prozesse sind Cluster, deren Bedeutung in den letzten Jahren gewachsen ist. Bei ihnen handelt es sich um endliche Systeme, die den Vorteil einer einfacheren theoretischen Behandlung bedingen [Ger94a]. Sie können als Modellsysteme für die flüssige und feste kondensierte Phase in Abhängigkeit von ihrer Größe betrachtet werden. In der Clusterumgebung eröffnen sich zudem andere Meßgrößen wie die kinetische Energie der auslaufenden Fragmente, die in Systemen der kondensierten Phase nicht zugänglich sind [Buc02]. 
Im Mittelpunkt dieser Arbeit steht die Messung photoinduzierter Dissoziationsprozesse von Halogenwasserstoffmolekülen in verschiedenen Clusterumgebungen, die hinreichend genau charakterisiert werden sollen. Das gilt sowohl für die Größe als auch für die genaue Lage des Moleküls im Cluster. Die zentrale Meßgröße stellt die kinetische Energie der auslaufenden Wasserstofffragmente dar, die genaue Auskunft über die sich abspielenden Prozesse gibt. In der Clusterumgebung können sich im Anschluß an die Dissoziation ionische, lineare Moleküle der Form Wasserstoff-Edelgas-Halogen ( $\mathrm{HRgX})$ bilden, deren Existenz bisher nur in Matrixexperimenten gezeigt wurde [Pet99].

Die Grundlagen der Photodissoziation insbesondere von Halogenwasserstoffen sowie der Käfigeffekt werden im ersten Kapitel diskutiert. Die Theorie der Düsenstrahlexpansion, Clustererzeugung und Clusterpräparation zeigt die Herstellung der zu untersuchenden Objekte auf. Die Flugzeitspektrometrie wird mit dem Fokus auf den energieaufgelösten Nachweis mittels resonant verstärkter Mehrphotonenionisation (REMPI) von Teilchen derselben Masse in einem Flugzeitmassenspektrometer mit Ortsfokussierung behandelt. Ebenfalls wird auf die Erzeugung von Wasserstoff-Edelgas-Halogen Verbindungen aus der Photodissoziation von Halogenwasserstoffen in Edelgasclusterumgebungen eingegangen. Die Voraussetzung zum Nachweis dieser Moleküle in dieser Apparatur ist deren Orientierung durch die Kombination eines starken, gepulsten Laserfeldes und eines schwachen, statischen elektrischen Feldes [Fri99b].

Das zweite Kapitel befaßt sich mit der experimentellen Versuchsanordnung. Die drei Bereiche Molekularstrahlerzeugung, Lasersysteme und Flugzeitmassenspektrometer werden in ihren wichtigsten Komponenten und Neuerungen gegenüber früheren Arbeiten beschrieben. Die Auswertung der Flugzeitspektren und deren Umwandlung in Energieverteilungen durch Lösung eines linearen Gleichungssystems findet im dritten Kapitel seine Beachtung. Dabei muß sich das für diese Umwandlung selbst entwickelte Simulationsprogramm gegenüber Experimenten bewähren, die durch Referenzmessungen und theoretische Berechnungen verifiziert sind.

Die experimentellen Ergebnisse dieser Arbeit werden im vierten Kapitel vorgestellt. Die Beschreibung erstreckt sich auf Photodissoziationsmessungen von $\mathrm{HI}, \mathrm{HBr}$ und $\mathrm{HCl}$ Molekülen in unterschiedlichen Edelgasclusterumgebungen. Der Nachweis der Moleküle $\mathrm{HXel}$ und $\mathrm{HXeCl}$ soll hier ebenfalls Erwähnung finden.

Die Diskussion der experimentellen Ergebnisse schließt sich in Kapitel 5 an. Der Vergleich der Messungen mit theoretischen Berechnungen aus den Gruppen von R.B. Gerber und $P$. Jungwirth gibt Aufschluß über die Position des Halogenwasserstoffmoleküls in der 
Clusterumgebung. Unterschiede im Vergleich zur Photodissoziation des freien Moleküls ergeben sich in der Photoanregung, den beteiligten Zuständen und der Bevölkerung der Produktkanäle. Daraus resultieren neue Erkenntnisse über Anregungs- und Kopplungsmechanismen der Photodissoziation von Halogenwasserstoffen in Lösungsmittelumgebungen. Den Abschluß dieser Arbeit bilden eine Zusammenfassung sowie ein Ausblick auf zukünftige Experimente. 



\section{Grundlagen}

Die wesentliche Grundlage für diese Arbeit bildet die Theorie der Photodissoziation und dort im speziellen die Photodissoziation von Halogenwasserstoffen HX. Die Photodissoziationsexperimente dieser Arbeit werden in Clusterumgebungen durchgeführt, so daß deren Einfluß in Form des Käfigeffekts diskutiert wird. Die Clustererzeugung geschieht in einer Düsenstrahlexpansion, wobei die Dotierung mit Chromophoren in unterschiedlichen Positionen in der Clusterumgebung verschiedene Techniken erfordert.

Die kinetische Energie der Wasserstofffragmente wird mit Hilfe der Flugzeitanalyse in einem Wiley-McLaren Flugzeitmassenspektrometer im Niedrigfeldmodus gemessen. Die lonisierung der nach der Photodissoziation neutralen Wasserstofffragmente geschieht mittels resonanter Mehrphotonenionisation, um diese im elektrischen Feld des Flugzeitmassenspektrometers abziehen zu können.

Die Photodissoziation in Clusterumgebungen eröffnet die Möglichkeit von Komplexbildungen. Dabei liegt hier das besondere Augenmerk auf Wasserstoff(H)-Edelgas(Rg)Halogen $(\mathrm{X})$ Verbindungen der Form $\mathrm{H}-\mathrm{Rg}-\mathrm{X}$. Diese Moleküle eignen sich aufgrund ihrer hohen Polarisierbarkeit zur Ausrichtung in Laserfeldern und wegen ihres permanenten Dipolmoments zur Orientierung in elektrostatischen Feldern. Hier soll insbesondere der Einfluß von kombinierten und Laser- und elektrostatischen Feldern auf diese Moleküle diskutiert werden.

\subsection{Photodissoziation}

Die Fragmentierung eines gebundenen Moleküls durch die Absorption eines oder mehrerer Photonen bezeichnet man als Photodissoziation. Bei der Absorption eines Photons wird dessen elektromagnetische Energie in innere Energie des Moleküls umgewandelt. Wenn die absorbierte Photonen-Energie die Energie der schwächsten Bindung übersteigt, wird die Molekülbindung gebrochen. Das Molekül liegt bei Erreichen des Dissoziationskontinuums 
als angeregter Komplex vor. Als Beispiel sei hier die Photodissoziation eines Moleküls $A B$ über den angeregten Zwischenzustand $(A B)^{*}$ in seine Produkte $A$ und $B$ illustriert. Die Produkte haben interne Freiheitsgrade $\alpha$ und $\beta, \mathrm{d}$.h. sie können nach der Dissoziation in einem angeregten Zustand vorliegen:

$$
\mathrm{AB}+N_{\text {photon }} h \nu \longrightarrow(\mathrm{AB})^{*} \longrightarrow \mathrm{A}(\alpha)+\mathrm{B}(\beta) .
$$

Dabei ist $h \nu$ die Energie eines Photons mit der Frequenz $\nu$ und $N_{\text {photon }}$ die Anzahl absorbierter Photonen.

In den Experimenten, die in dieser Arbeit dargestellt sind, wird für die Dissoziation ein einziges Photon absorbiert. Die Entwicklung leistungsstarker UV-Laser in den letzten beiden Jahrzehnten hat dazu beigetragen, daß die meisten Moleküle in einem Einphotonenprozeß dissoziiert werden können. Das Hauptaugenmerk dieser Experimente liegt auf der Bestimmung der kinetischen Energie der Produkte und deren interner Energien als Funktion des Anfangszustandes des Ausgangsmoleküls.

Die wichtigste Größe in der Photodissoziation ist der Absorptionsquerschnitt des Moleküls für Photonen unterschiedlicher Frequenzen. An ihm läßt sich ablesen, ob die Photodissoziation stattfindet, und wenn, wie effektiv. Der totale Absorptionsquerschnitt $\sigma(\nu)$ setzt sich aus den partiellen Querschnitten der einzelnen Produktkanäle $\sigma\left(\nu, \gamma_{i}\right)$ zusammen. Der Anteil eines Produktkanals am totalen Querschnitt wird durch den Verzweigungsanteil $\Gamma$ (branching fraction) ausgedrückt,

$$
\Gamma\left(\nu, \gamma_{1} \ldots \gamma_{j} \ldots \gamma_{n}\right)=\frac{\sigma\left(\nu, \gamma_{j}\right)}{\sum_{i=1}^{n} \sigma\left(\nu, \gamma_{i}\right)} .
$$

Bei nur zwei Produktkanälen benutzt man zur Beschreibung häufig das Verzweigungsverhältnis $R$ (branching ratio):

$$
R\left(\nu, \gamma_{1}, \gamma_{2}\right)=\frac{\sigma\left(\nu, \gamma_{1}\right)}{\sigma\left(\nu, \gamma_{2}\right)}
$$

Weitere Meßgrößen sind die Verteilungen von Schwingungs- und Rotationszuständen bei mehratomigen Dissoziationsfragmenten. Die bisher aufgeführten Meßgrößen sind alle skalarer Natur, wobei die Photodissoziation ein vektorieller Prozeß ist. Dieser kann durch mehrere Vektorkorrelationen beschrieben werden. Die Polarisation des elektrischen Feldes $\vec{E}_{0}$ definiert eine einheitliche Richtung, auf die sich alle anderen Vektoren beziehen, sowohl diejenigen, die das Ausgangsmolekül, als auch die, die die Fragmente beschreiben: 
- $\vec{\mu}$ : Übergangsdipolmoment des Ausgangsmoleküls

- $\vec{v}$ : Rückstoßgeschwindigkeit der Fragmente

- $\vec{\jmath}$ : Drehimpuls der Fragmente.

Daraus resultieren folgende Vektorkorrelationen:

- $\overrightarrow{\boldsymbol{E}}_{\mathbf{0}}-\overrightarrow{\boldsymbol{\mu}}-\overrightarrow{\boldsymbol{v}}$ Korrelation: Das elektrische Feld $\vec{E}_{0}$ richtet das Ausgangsmolekül mit seinem körperfesten Übergangsdipolmoment aus (alignment). Die Verteilung der ausgerichteten Moleküle ist proportional zu $\cos ^{2} \theta$, wobei $\theta$ den Winkel zwischen $\vec{\mu}$ und $\vec{E}_{0}$ beschreibt. Bei direkter Dissoziation wird diese Ausrichtung eine Anisotropie der Fragmentverteilung zur Folge haben. Es gibt allerdings Effekte, die zum Verlust der Ausrichtung des Ausgangsmoleküls führen können. Dazu zählen Rotationen, die schneller ablaufen als der Dissoziationsprozeß und Stöße mit Teilchen in der Umgebung, wie sie z. B. im Cluster auftreten. Die $\vec{E}_{0}-\vec{\mu}-\vec{v}$ Korrelation bezieht sich auf das Laborsystem.

- $\overrightarrow{\boldsymbol{E}}_{\mathbf{0}}-\overrightarrow{\boldsymbol{\mu}}-\overrightarrow{\boldsymbol{\jmath}}$ Korrelation: Diese Korrelation tritt nur für drei- und mehratomige Moleküle auf, bei deren Photodissoziation mehratomige Fragmente entstehen, die wiederum einen Drehimpuls $\vec{\jmath}$ besitzen. Bei quantenmechanischer Behandlung des Systems ist die Projektion von $\vec{\jmath}$ auf die durch $\vec{E}_{0}$ definierte Achse quantisiert mit den Quantenzahlen $m_{j}=-j,-j+1, \ldots, j-1, j$. Die Verteilung der Quantenzahlen $m_{j}$ läßt darauf schließen, ob ein Molekül ausgerichtet oder orientiert ist. Bei einem orientierten Molekül ist die Verteilung von $m_{j}$ asymmetrisch zu $m_{j}=0$. Ausgerichtete Moleküle zeigen zu gleichen Teilen parallel wie antiparallel zum Feldstärkevektor $\vec{E}_{0}$. Die Verteilung ist symmetrisch um $m_{j}=0$. Weiteres zur Ausrichtung (alignment) und Orientierung (orientation) von Molekülen findet sich in Abschnitt 1.4.2. Auch diese Korrelation bezieht sich auf das Laborsystem.

- $\vec{v}-\vec{\jmath}$ Korrelation: Im Gegensatz zu den beiden anderen Korrelationen ist diese völlig unabhängig vom Laborsystem. Sie bezieht sich nur auf das Schwerpunktsystem. Dies eröffnet in der Untersuchung des Photodissoziationsprozesses eine von der Ausrichtung und dem Ausrichtungsgrad des Ausgangsmoleküls unabhängige Meßgröße. Aufgrund der Beteiligung des Drehimpulses des Fragments $\vec{\jmath}$ tritt sie nur in polyatomaren Molekülen auf. In der $\vec{v}-\vec{\jmath}$ Korrelation wird die Ausrichtung der Geschwindigkeit $\vec{v}$ zum eigenen Drehimpuls $\vec{\jmath}$ betrachtet. 
Im folgenden spielt nur die $\vec{E}_{0}-\vec{\mu}-\vec{v}$ Korrelation eine Rolle, weil in dieser Arbeit nur die Photodissoziation zweiatomiger Moleküle behandelt wird.

\subsubsection{Photodissoziation zweiatomiger Moleküle}

Die Photodissoziation zweiatomiger Moleküle kann als Prototyp betrachtet werden, weil sie sich in theoretischen Betrachtungen am besten behandeln läßt. In der Dissoziation entstehen nur zwei Fragmente. Die Messung der Energie des einen Fragments liefert die des anderen über die Energie- und Impulserhaltung. Eine weitere Vereinfachung tritt für die Position des Übergangsdipolmoments $\vec{\mu}$ auf, das nur parallel $\left(\chi=0^{\circ}\right)$ oder senkrecht $\left(\chi=90^{\circ}\right)$ zur Kernverbindungsachse der beiden Atome gerichtet sein kann.

In diesen Experimenten ist die räumliche Fragmentverteilung $F(\vec{v})$ die zugängliche Meßgröße. Sie läßt sich in ein Produkt aus einem skalaren, geschwindigkeitsabhängigen Term (Geschwindigkeitsverteilung $G(|\vec{v}|)$ ) und einem winkelabhängigen Term (Winkelverteilung $I(\theta))$ zerlegen.

$$
F(\vec{v})=G(|\vec{v}|) \cdot I(\theta)
$$

\subsubsection{Energiebilanz}

Die Messung der Verteilung der Photofragmente erlaubt die Bestimmung der kinetischen Energie der Fragmente. Die Energiebilanz der Photodissoziation eines freien Moleküls setzt sich wie folgt zusammen:

$$
h \nu+E_{\text {int }}(\mathrm{AB})-D_{0}=E_{k i n}(\mathrm{~A})+E_{\text {int }}(\mathrm{A})+E_{k i n}(\mathrm{~B})+E_{\text {int }}(\mathrm{B}) \quad .
$$

Dabei bezeichnet $h \nu$ die Energie des absorbierten Photons mit der Frequenz $\nu$. Das Molekül AB kann zum Zeitpunkt der Dissoziation durch Rotations-, Vibrations- oder elektronische Anregung über innere Energie verfügen $\left(E_{\text {int }}(\mathrm{AB})\right)$. $D_{0}$ bezeichnet die Dissoziationsenergie des Moleküls AB. Auf der Produktseite finden sich die kinetischen $\left(E_{k i n}(\mathrm{~A}), E_{k i n}(\mathrm{~B})\right)$ und internen $\left(E_{\text {int }}(\mathrm{A}), E_{\text {int }}(\mathrm{B})\right)$ Energien der Dissoziationsfragmente. Dabei ist bei Messung der kinetischen Energie eines Fragments die des anderen über die Impulserhaltung bekannt. Innere Energien der Photofragmente sind elektronische Anregungen der Atome.

In diesen Experimenten werden die Moleküle durch Düsenstrahlexpansion in einem Molekularstrahl präpariert. Bei dieser Methode besitzen die sehr kalten Moleküle weder 
Schwingungs- noch Rotationsanregung. Durch spektroskopische Messungen aus der Literatur sind die Dissoziationsenergien zweiatomiger Moleküle sehr gut bekannt. Dies führt in der Auswertung der Energiebilanz zu einer deutlichen Vereinfachung.

\subsubsection{Winkelverteilung}

Die Winkelverteilung $I(\theta)$ resultiert ausschließlich aus der $\vec{E}_{0}-\vec{\mu}-\vec{v}$ Vektor-Korrelation. Im Spezialfall des zweiatomigen Moleküls zeigt $\vec{\mu}$ parallel oder senkrecht zur Kernverbindungsachse und $\theta$ beschreibt den Winkel zwischen $\vec{E}_{0}$ und $\vec{\mu}$ (s. Abb. 1.1).

parallel transition

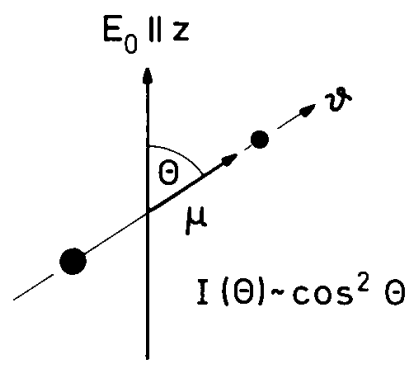

perpendicular transition

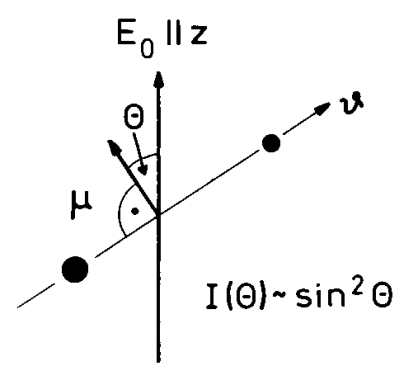

Abbildung 1.1: Winkelverteilung bei der Photodissoziation zweiatomiger Moleküle: Illustration der $\vec{E}_{0}-\vec{\mu}-\vec{v}$ Vektor-Korrelation. Die Polarisation des elektrischen Feldvektors $\vec{E}_{0}$ ist parallel zur Detektorachse z. Gezeigt ist die Ausrichtung des Moleküls infolge eines parallelen (linkes Bild) und eines senkrechten (rechtes Bild) Übergangs. Das Bild ist entnommen aus [Sch93]

Aus der Entwicklung des elektrischen Dipolmoments erhält man für die Übergangswahrscheinlichkeit eine Proportionalität in $\cos ^{2} \theta$ [Sch93]. Bevorzugt regt das Photon Moleküle an, deren Übergangsdipolmoment parallel zum Polarisationsvektor ausgerichtet ist. Dies führt direkt nach Absorption des Photons zu einer Ausrichtung des angeregten Moleküls im Laborsystem.

Normalerweise führt die Winkelverteilung des Moleküls auch zu einer Winkelverteilung der Rückstoßgeschwindigkeit $\vec{v}$ der Fragmente. Unter der Voraussetzung, daß sich die Dissoziation des Moleküls schnell abspielt im Vergleich zu Rotationen $\left(\tau_{\text {diss }} \ll \tau_{\text {rot }}\right)^{1}$, ist die Rückstoßgeschwindigkeit parallel zur Kernverbindungsachse. Wenn der Dissoziationsprozeß

\footnotetext{
${ }^{1}$ Die Zeitskala, auf der sich Dissoziationen nach einer Photonenanregung abspielen, liegt in der Größenordnung von $10 \mathrm{fs}$. Für $\mathrm{HI}$ dauert sie je nach Produktkanal zwischen 15 und $45 \mathrm{fs}$ [Bal01].
} 
sich sofort nach der Absorption eines Photons abspielt, resultiert daraus eine Winkelverteilung der Fragmente $I(\theta)$, die für parallele Übergänge proportional zu $\cos ^{2} \theta$ und für senkrechte proportional zu $\sin ^{2} \theta$ ist. Allgemein schreibt man für die Winkelverteilung

$$
I(\theta)=\frac{\sigma_{0}}{4 \pi}\left[1+\beta P_{2}(\cos \theta)\right],
$$

mit $P_{2}(x)=\frac{1}{2}\left(3 x^{2}-1\right)$, Legendre Polynom 2. Ordnung und dem Anisotropiefaktor $\beta$. Der Anisotropiefaktor, auch Anisotropieparameter genannt, nimmt Werte zwischen -1 für senkrechte und 2 für parallele Übergänge an. Die Daten für parallele und senkrechte Übergänge sind in Gl. 1.7 zusammengestellt. Dabei beschreibt $\chi$ den Winkel zwischen dem Übergansgsdipolmoment $\vec{\mu}$ und der Kernverbindungsachse. $\Delta \Lambda$ und $\Delta \Omega$ stehen für die mit den Kopplungsfällen $(\Lambda, \Sigma)$ und $\left(\Omega_{c}, \omega\right)$ verknüpften Auswahlregeln ${ }^{2}$ [Her91]:

$$
\begin{array}{lllll}
\text { parallel } & \chi=0^{\circ} & \Delta \Lambda, \Omega=0 & \beta=2 & I(\theta)=\frac{3}{4 \pi} \cos ^{2} \theta \\
\text { senkrecht } & \chi=90^{\circ} & \Delta \Lambda, \Omega= \pm 1 & \beta=-1 & I(\theta)=\frac{3}{8 \pi} \sin ^{2} \theta .
\end{array}
$$

Die geometrische Form der Winkelverteilung im Raum für einen senkrechten Übergang, einen parallelen Übergang und zum Vergleich eine isotrope Verteilung sind in Abb. 1.2 dargestellt. Für einen senkrechten Übergang erhält man eine äquatoriale für einen parallelen eine Polkappenverteilung der Fragmente [Kin92].

Aus der Messung der Fragmentwinkelverteilung lassen sich Informationen über elektronische Übergänge im Ausgangsmolekül und infolgedessen auch über die Symmetrie von angeregten Zuständen gewinnen [Ber69, Zar72, Bus72, Zar88, Sch93].

\subsubsection{Photodissoziation von Halogenwasserstoffen}

Unter chemischen Aspekten könnte man davon ausgehen, daß die Halogenwasserstoffe ionisch gebundene Moleküle sind. In einer Lösung dissoziiert $\mathrm{HCl}^{\text {in } \mathrm{H}^{-}}$und $\mathrm{Cl}^{+}$. Die Differenz $I(\mathrm{~A})-E(\mathrm{~B})=9.88 \mathrm{eV}^{3}$ läßt allerdings eher auf eine kovalente Bindung schließen. Mit der Annahme zweier Punktladungen in einem ionisch gebundenen Molekül ergibt sich ein

\footnotetext{
${ }^{2}$ Bahndrehimpuls und Gesamtspin ergeben die Quantenzahl $\Omega$ als Summe von $\Lambda$ und $\Sigma$, d.h. als Summe der Quantenzahlen und damit als Projektion auf die Kernverbindungslinie. $\Delta \Lambda$ und $\Delta \Omega$ beschreiben die Änderung der Quantenzahlen.

${ }^{3} I(A)-E(B)$ gibt für ein Molekül $\mathrm{AB}$ die energetische Differenz zwischen dem niedrigsten ionischen Zustand $\mathrm{A}^{+}+\mathrm{B}^{-}$und dem niedrigsten atomaren Zustand $\mathrm{A}+\mathrm{B}$ an. $I(\mathrm{~A})$ : lonisierungspotential von $\mathrm{A} ; E(\mathrm{~B})$ : Elektronenaffinität von $\mathrm{B}$.
} 

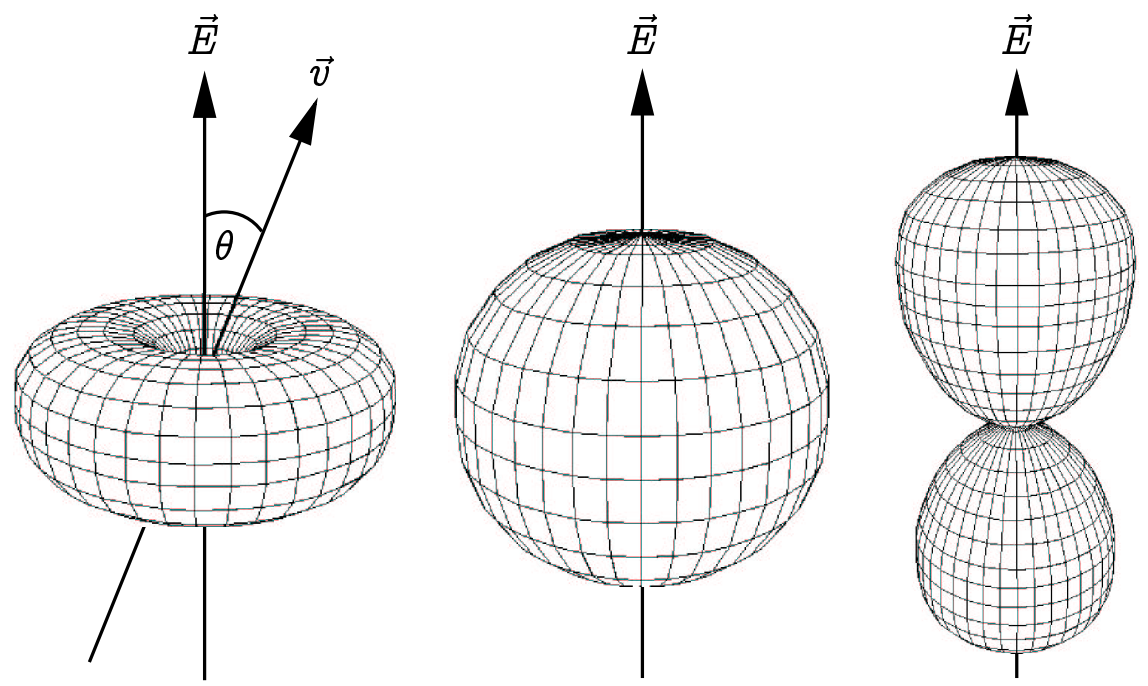

Abbildung 1.2: Winkelverteilung der Fragmente bei der Photodissoziation. Vergleich zwischen einem senkrechten Übergang $\left(I(\theta) \propto \sin ^{2} \theta, \beta=-1\right.$, links) und einem parallelen $(I(\theta) \propto$ $\cos ^{2} \theta, \beta=2$, rechts). Der mittlere Teil zeigt eine isotrope Verteilung $(\beta=0)$.

Abstand von $r=1.45 \AA$; $\left(V=-\epsilon^{2} / r\right)$. Der lonenradius von $\mathrm{Cl}^{-}$beträgt $1.8 \AA$, währenddessen der Kernabstand von $\mathrm{HCl}$ nur $1.27 \AA ̊$ beträgt. Daraus folgt, daß $\mathrm{HCl}$ im gasförmigen Zustand eine kovalente Bindung besitzt. Dieses Ergebnis läßt sich auf die anderen Wassestoffhalogenide übertragen [Her91, Mul37].

Bei der Anregung von Wasserstoffhalogeniden in das erste Absorptionskontinuum ( $A$ Band) dissoziieren alle Spezies $\mathrm{HX}(\mathrm{X}=\mathrm{F}, \mathrm{Cl}, \mathrm{Br}, \mathrm{I})$ unmittelbar in zwei mögliche Produktkanäle:

$$
\begin{aligned}
\mathrm{HX}+h \nu & \longrightarrow \mathrm{H}\left({ }^{2} S\right)+\mathrm{X}\left({ }^{2} P_{3 / 2}\right) \\
& \longrightarrow \mathrm{H}\left({ }^{2} S\right)+\mathrm{X}\left({ }^{2} P_{1 / 2}\right)
\end{aligned}
$$

Der $\mathrm{X}\left({ }^{2} P_{3 / 2}\right)$ Grundzustand des Halogenatoms wird nach Konvention mit $\mathrm{X}$ gekennzeichnet und der $\mathrm{X}\left({ }^{2} P_{1 / 2}\right)$ angeregte Zustand mit $\mathrm{X}^{*}$. Die Anregung resultiert aus der Spin-Bahn Aufspaltung im Halogenatom. Als charakteristische Größe in Messungen und Rechnungen wird das Verzweigungsverhältnis $R=\left[\mathrm{X}^{*}\right] /[\mathrm{X}]$ zwischen diesen beiden Zuständen nach $\mathrm{Gl}$. 1.3 ermittelt. 
Die Photodissoziation von Halogenwasserstoffen über die Anregung des $A$ Absorptionsbandes und die Ermittlung des Verzweigungsverhältnisses waren in den letzten Jahrzehnten Gegenstand einer Vielzahl von theoretischen und experimentellen Untersuchungen (HF: [Zha96], HCl: [Reg00, Asc99, Lam98, Ale98, Bor98, Che02, Zha97, Liy95, Duh95, Ger94b, Ale93, Mat92], HBr: [Reg99b, Bau99b, Pou98, Peo97, Xu88, Nee86], HI: [Roy02, Bal01, Ale00, Reg99a, Lan98, Gen98, Xu88, Van83, Sch82]). Die ersten quantenmechanischen Berechnungen zu Absorptionsquerschnitten, Produktkanälen und elektronischen Zuständen gehen auf die Pionierarbeiten von Mulliken in den 30er Jahren des letzten Jahrhunderts zurück [Mul37, Mul40]. Aufgrund ihrer relativ einfachen Elektronenkonfiguration der Fragmente $\left(\mathrm{H}: 1 \mathrm{~s}^{1}, \mathrm{X}: \mathrm{ns}^{2} \mathrm{np}^{5}\right)$ sind die Wasserstoffhalogenide theoretischen Berechnungen gut zugänglich.

Insgesamt sind an der Photodissoziation von Wasserstoffhalogeniden zwölf elektronische Zustände beteiligt. Die Kennzeichnung dieser Zustände richtet sich nach dem Hund'schen Fall (a), wobei für HX die Spin-Bahn Wechselwirkung nicht vernachlässigbar ist. Die Zustände werden zum einen durch ihre Multiplizität $(2 S+1)$, wobei $S$ der elektronische Gesamtspin ist, gekennzeichnet, zum anderen durch den Gesamtdrehimpuls $J$, der sich aus den Quantenzahlen $\Lambda$ und $\Omega$ zusammensetzt. Diese Quantenzahlen sind Projektionen des elektronischen Drehimpulses $\vec{L}(\Lambda)$ und des elektronischen Gesamtdrehimpulses $\vec{J}=\vec{L}+\vec{S}(\Omega)$ auf die Kernverbindungsachse. Im weiteren ist noch die Parität der Zustände zu unterscheiden. Es gibt die e Zustände mit der Parität $+(-1)^{J}$ und die f Zustände mit der Parität $-(-1)^{J}$. Die Quantenzahl $J$ ist in diesem Fall ganzzahlig. Falls keine externen elektrischen Felder anliegen, findet zwischen den $\mathbf{e}$ und $\mathbf{f}$ Zuständen sowie zwischen Zuständen mit unterschiedlichem $J$ keine Kopplung statt. Es gibt jeweils sechs e $\left({ }^{3} \Sigma_{1}^{+},{ }^{3} \Pi_{\Omega=0,1,2},{ }^{1} \Sigma_{0}^{+}\right.$und $\left.{ }^{1} \Pi_{1}\right)$ und $\mathbf{f}\left({ }^{3} \Sigma_{1}^{+},{ }^{3} \Pi_{\Omega=0,1,2},{ }^{3} \Sigma_{0}^{+}\right.$und $\left.{ }^{1} \Pi_{1}\right)$ Zustände. [Zar88, Bro74, Ale93]. Die adiabatischen Potentialflächen der zwölf Zustände sind in Abb. 1.3 in Abhängigkeit vom Kernabstand skizziert.

Die Auswahlregeln eines elektronischen Dipolübergangs $\Delta J=0, \pm 1$ und $+\leftrightarrow-$ werden damit in der e,f Notation zu

$$
\begin{array}{ll}
\text { Q-Zweig, d. h.: } & \Delta J=\quad 0, \quad \mathbf{e} \leftrightarrow \mathbf{f} \quad, \\
\text { P-, R-Zweig, d.h.: } & \Delta J=\quad \mp 1, \quad \mathbf{e} \leftrightarrow \mathbf{e} \quad \text { und } \quad \mathbf{f} \leftrightarrow \mathbf{f} .
\end{array}
$$

Je nach Halogen werden die zur Dissoziation führenden Potentialflächen drei oder vier repulsiven elektronischen Zuständen zugeschrieben. Mit $X^{1} \Sigma_{0}^{+}$als Grundzustand ergeben sich folgende mögliche Anregungen: 


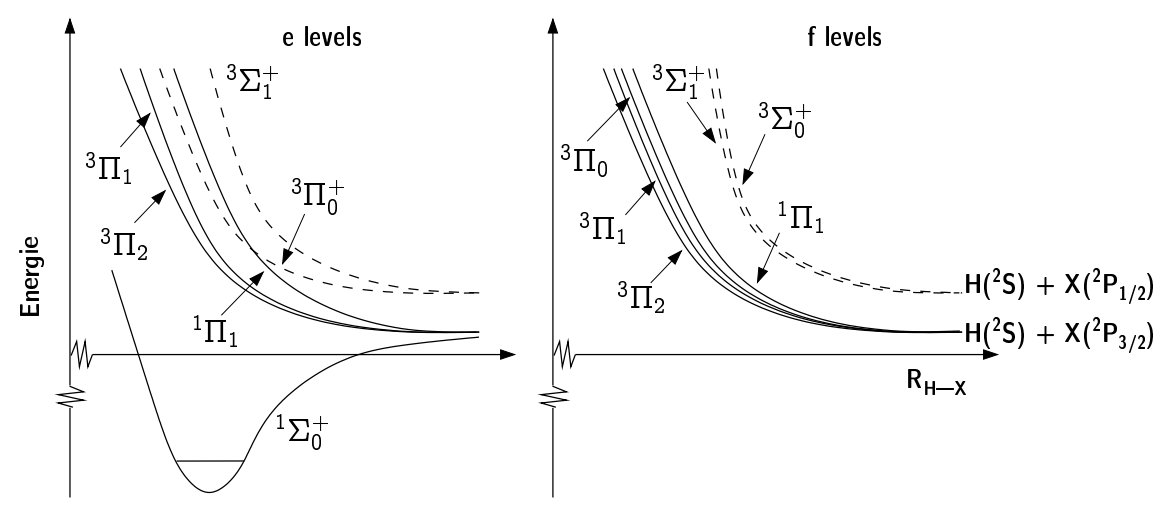

Abbildung 1.3: Schematische Darstellung der zwölf Potentialkurven, die an der Photodissoziation von Halogenwasserstoffen beteiligt sind. $\mathrm{R}_{\mathrm{H}-\mathrm{X}}$ bezeichnet den Abstand zwischen dem Wasserstoffatom $\mathrm{H}$ und dem Halogenatom $\mathrm{X}(\mathrm{X}=\mathrm{Cl}, \mathrm{Br}, \mathrm{I})$. Es gibt zwei Produktkanäle. Der erste führt in den Grundzustand des Halogenatoms $\mathrm{H}\left({ }^{2} \mathrm{~S}\right)+$ $\mathrm{X}\left({ }^{2} \mathrm{P}_{3 / 2}\right)$, der zweite in den um die Spin-Bahn-Aufspaltung höheren angeregten $\mathrm{Zu}$ stand des Halogenatoms $\left(\mathrm{H}\left({ }^{2} \mathrm{~S}\right)+\mathrm{X}\left({ }^{2} \mathrm{P}_{1 / 2}\right)\right)$. Zwischen den Kanälen sind je nach beteiligtem Halogen und Anregungswellenlänge Kopplungen mit unterschiedlichen Mechanismen möglich.

$$
\begin{array}{lllll}
A^{1} \Pi_{1} \leftarrow X^{1} \Sigma_{0}^{+} & \text {senkrecht } \Delta \Omega= \pm 1 \rightarrow \mathrm{X} & \text { bei } \mathrm{HCl}, \mathrm{HBr}, \mathrm{HI} \\
a^{3} \Pi_{1} \leftarrow X^{1} \Sigma_{0}^{+} & \text {senkrecht } \Delta \Omega= \pm 1 \rightarrow \mathrm{X} & \text { bei } \mathrm{HBr}, \mathrm{HI} \\
a^{3} \Pi_{0}^{+} \leftarrow X^{1} \Sigma_{0}^{+} & \text {parallel } \Delta \Omega=0 \rightarrow \mathrm{X}^{*} & \text { bei } \mathrm{HCl}, \mathrm{HBr}, \mathrm{HI} \\
t^{3} \Sigma_{1}^{+} \leftarrow X^{1} \Sigma_{0}^{+} & \text {senkrecht } \Delta \Omega= \pm 1 \rightarrow \mathrm{X}^{*} \text { bei } \mathrm{HI} .
\end{array}
$$

Die beiden ersten Übergänge laufen asymptotisch in den Grundzustand X, während die beiden unteren mit dem angeregten Zustand $X^{*}$ korreliert sind. Ohne Kopplungen kann der Grundzustand keine parallelen Anteile enthalten. Die direkte Anregung in den ${ }^{3} \Sigma_{1}^{+} \mathrm{Zu}$ stand spielt nach aktueller Erkenntnis nur bei der Photodissoziation von $\mathrm{HI}$ und DI bei der Verwendung kurzer Wellenlängen eine Rolle [Roy02]. Beim Übergang auf den repulsiven Potentialflächen in die Produktkanäle findet je nach Halogen und Anregungswellenlänge durch nicht-adiabatische Kopplungen (Rotations-, Spin-Bahn Kopplungen) eine Umverteilung des Photodissoziationsflusses in andere Zustände statt. An der Photodissoziation können demnach noch andere als die oben aufgeführten fünf Niveaus beteiligt sein.

Das Interesse an den Wasserstoffhalogeniden ist nicht überraschend, weil diese zweiatomigen Moleküle als Modellsysteme für die Analyse von Photodissoziationsprozessen mit 
mehreren beteiligten Produktkanälen dienen. Das wiederum hilft im Verständnis der Photofragmentation von polyatomaren Molekülen wie $\mathrm{CH}_{3} \mathrm{Cl}$ [Lin02] oder $\mathrm{CF}_{3} \mathrm{I}$. Ebenso bieten sie die Möglichkeit, leichte Systeme wie $\mathrm{HF}$ und $\mathrm{HCl}$ mit schwereren isovalenten Systemen $(\mathrm{HBr}$ und $\mathrm{HI})$ zu vergleichen, und deren Beteiligung an der Spin-Bahn Kopplung im Dissoziationsprozeß zu untersuchen. Letztere führt zu deutlichen Unterschieden in der optischen Anregung verschiedener Dissoziationskanäle und beeinflußt damit die energetischen Eigenschaften der dissoziativen Potentialflächen. Die Kopplung zwischen den Potentialen führt dann zu unterschiedlichen Verteilungen in die Produktkanäle und damit zu anderen Verzweigungsverhältnissen.

Experimentell hat man die Möglichkeit, ein Wasserstoffhalogenid mit Licht unterschiedlicher Wellenlängen (Energien) in das Dissoziationskontinuum anzuregen. Dabei ist zum einen der Photodissoziationsquerschnitt wellenlängenabhängig, zum anderen setzt sich dieser aus den Querschnitten der einzelnen Zustände zusammen. Das bedeutet, daß man bei Anregung mit unterschiedlichen Wellenlängen verschiedene Zustände bevölkert, was dann wiederum zu einer Änderung des Verzweigungsverhältnisses der Produktkanäle führt. Wenn die Untersuchungen mit polarisiertem Licht durchgeführt werden, kann sich in Abhängigkeit von der Anregungswellenlänge auch die Winkelverteilung der Fragmente verändern, weil die Anregung entweder über einen parallelen oder einen senkrechten Übergang abläuft. Auch Kopplungen lassen sich auf diese Weise experimentell bestimmen.

Im folgenden sollen die individuellen Eigenschaften der einzelnen Wasserstoffhalogenide $\mathrm{HCl}, \mathrm{HBr}$ und $\mathrm{HI}$ betrachtet werden. Dazu können die in Abb. 1.4 gezeigten Absorptionsquerschnitte mit herangezogen werden. Die Kurve für $\mathrm{HCl}$ basiert auf theoretischen Berechnungen aus der Gruppe von B. Pouilly [Ale93]. Die Kurve von $\mathrm{HBr}$ basiert auf Absorptionsmessungen mit Synchrotronstrahlung von L.C. Lee. Die Zuordnung der beteiligten Zustände beruhen auf theoretischen Betrachtungen von B. Pouilly [Pou98] und S.H. Lin [Cha87]. Der Absorptionsquerschnitt von HI war Gegenstand einer Vielzahl von experimentellen und theoretischen Arbeiten in den letzten Jahrzehnten. R.J. Le Roy hat aus allen Arbeiten zu HI und DI den Absorptionsquerschnitt von $\mathrm{HI}$ ermittelt [Roy02].

\section{$\mathrm{HCl}$}

An der Photodissoziation von $\mathrm{HCl}$ sind insgesamt fünf der zwölf Zustände beteiligt. Die Zustände ${ }^{1,3} \Sigma^{+}$und ${ }^{1,3} \Pi$ sind repulsiv, nur $X^{1} \Sigma^{+}$ist gebunden. Die Dissoziation läuft 

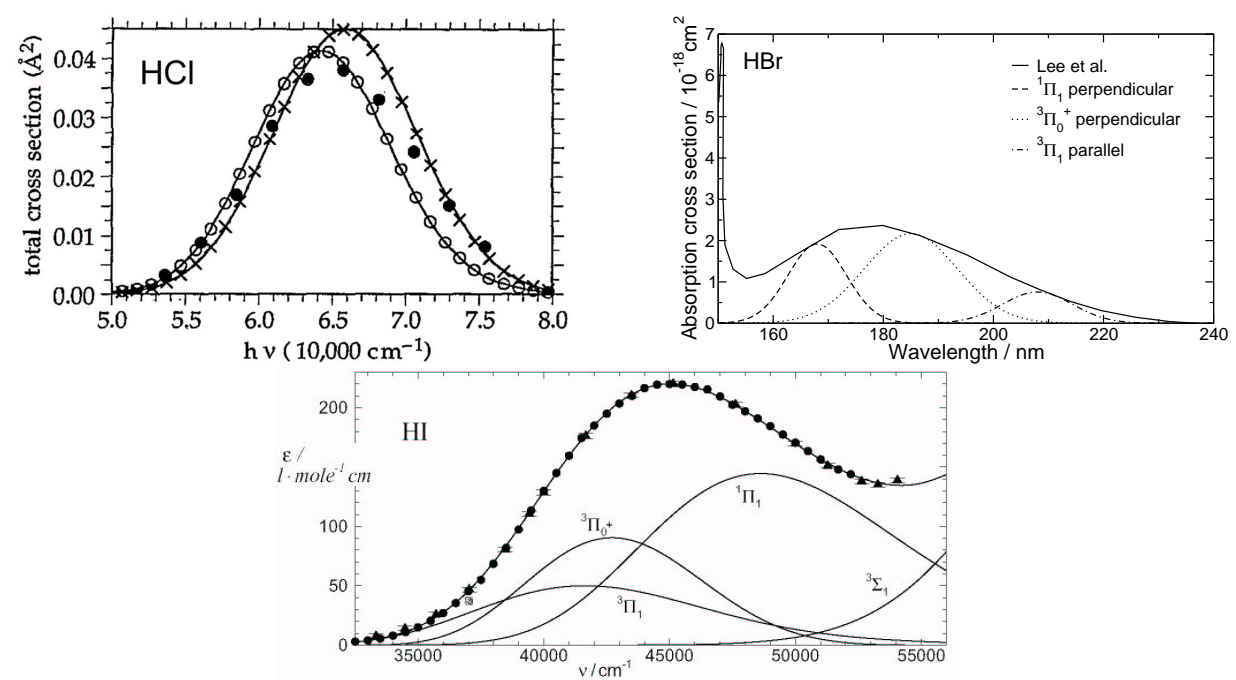

Abbildung 1.4: Photonen-Absorptionsquerschnitte der Halogenwasserstoffe, oben links $\mathrm{HCl}$ [Ale93], oben rechts $\mathrm{HBr}$ [Nee86, Pou98, Cha87], unten $\mathrm{HI}$ [Roy02]. Umrechnung der in den Experimenten verwendeten Wellenlängen: $243 \mathrm{~nm} \rightarrow 41228 \mathrm{~cm}^{-1} \rightarrow$ $5.11 \mathrm{eV}, 193 \mathrm{~nm} \rightarrow 51910 \mathrm{~cm}^{-1} \rightarrow 6.43 \mathrm{eV}$

über die senkrechte Anregung des repulsiven $A^{1} \Pi_{1}$ Zustandes im Franck-Condon Bereich ${ }^{4}$ $2.23 \leq R \leq 2.66$ bohr ab. ${ }^{5}$ Die $\Omega=1$ Komponenten der anderen Zustände $\left(a^{3} \Pi_{1}, t^{3} \Sigma^{+}\right)$ werden im Dissoziationsprozeß über nicht-adiabatische Spin-Bahn Kopplung bevölkert. Dieser Mechanismus dominiert die Wechselwirkung bei großen Kernabständen $R$, wobei sich die elektronischen Zustände verbinden [Ale93]. Relevante Kopplung in den $t^{3} \Sigma^{+}$Zustand findet bei einem Abstand von $R \approx 4$ bohr statt, in den $a^{3} \Pi_{1}$ bei $R \approx 6-8$ bohr [Reg00]. $\mathrm{Daß}$ nur der $A^{1} \Pi_{1}$ Zustand in der Anregung bevölkert wird, ist darauf zurückzuführen, daß in einem leichten Molekül wie $\mathrm{HCl}$ die Spin-Bahn Wechselwirkungen im Franck-Condon Bereich sehr klein sind. Daraus folgt, daß nur Spin-erhaltende Übergänge erlaubt sind.

Neuere Messungen von Wittig et al. [Zha97] ergeben bei einer Dissoziationswellenlänge

${ }^{4}$ Franck-Condon-Prinzip: Die Absorption vom Schwingungsgrundzustand ist für denjenigen Übergang in einen Schwingungsterm eines höheren elektronischen Zustandes am stärksten, der in der Potentialdarstellung mit dem Maximum seiner Wellenfunktion senkrecht darüber liegt. Übergänge zu anderen Schwingungstermen sind ebenfalls möglich, aber mit geringerer Wahrscheinlichkeit.

${ }^{5}$ Ein kleiner Teil $(0.5-0.8 \%)$ kann bei großen Wellenlängen den repulsiven $a^{3} \Pi_{0+}$ Zustand bevölkern. Hierbei handelt es sich um einen parallelen Übergang $\Delta \Omega=0$. Die Potentialkreuzung mit $A^{1} \Pi_{1}$ führt zu keiner Kopplung [Ale93, Zha97]. 
von $193 \mathrm{~nm}$ Anistotropiefaktoren $\beta(\mathrm{Cl})=-1.01$ und $\beta\left(\mathrm{Cl}^{*}\right)=-0.94$. Das Verzweigungsverhältnis liegt bei $\mathrm{R}=0.69(\mathrm{R}(157 \mathrm{~nm})=0.88$ [Mat90, Mat92, Ton92]). Der senkrechte Übergang in den Zustand $A^{1} \Pi_{1}$ führt in den Produktkanal $\left.\left.\mathrm{H}^{2} \mathrm{~S}\right)+\mathrm{Cl}^{2} \mathrm{P}_{3 / 2}\right)$, während durch Kopplung mit dem senkrechten Übergang $t^{3} \Sigma^{+}$eine Umverteilung in den angeregten Zustand $\mathrm{Cl}^{*}$ stattfindet. Der nur schwach beteiligte parallele Übergang in $a^{3} \Pi_{0^{+}}$führt in den angeregten Zustand $\mathrm{Cl}^{*}$. Er koppelt nicht mit den anderen Zuständen. Der Anteil des parallelen Übergangs sorgt dafür, daß $\beta\left(\mathrm{Cl}^{*}\right)$ etwas größer als -1 ist.

\section{$\mathrm{HBr}$}

Im Gegensatz zu HCl ist die Anregung einer repulsiven Potentialfläche bei $\mathrm{HBr}$ abhängig von der Wellenlänge (s. Abb. 1.4). Beteiligt sind die senkrechten Anregungen in die Zustände $A^{1} \Pi_{1}$ und $a^{3} \Pi_{1}$ sowie die parallele Anregung in $a^{3} \Pi_{0^{+}}$. Weitere Übergänge sind unter Berücksichtigung der Spin-Bahn Kopplung durch Photoanregung im Franck-Condon Bereich nicht zugänglich. Durch die wellenlängenabhängige Anregung und Kopplungen zum ${ }^{3} \Sigma_{1}^{+}$Zustand verändern sich auch das Verzweigungsverhältnis und die Anisotropie der Dissoziation.

In früheren Arbeiten unserer Gruppe wurden die Winkelverteilung und das Verzweigungsverhältnis in der Photodissoziation von $\mathrm{HBr}$ Molekülen bei Anregungswellenlängen von 193 und $243 \mathrm{~nm}$ experimentell bestimmt [Bau97a, Bau97b, Fri97, Nah98, Bau99b]. Die Dissoziation in den $\mathrm{Br}$ Kanal verläuft in beiden Fällen überwiegend senkrecht $\left(\beta_{243 \mathrm{~nm}}=\right.$ $-0.96 \pm 0.05, \beta_{193 \mathrm{~nm}}=-0.90 \pm 0.1$ ), während im Ausgangskanal $\mathrm{Br}^{*}$ ein Wechsel stattfindet. Bei großen Wellenlängen besitzt die Dissoziation überwiegend parallelen Charakter $\left(\beta_{243 \mathrm{~nm}}^{*}=1.96 \pm 0.05\right)$. Hin zu niedrigeren Wellenlängen findet eine Anregung über senkrechte Übergänge statt, die durch Kopplung in den ${ }^{3} \Sigma_{1}^{+}$Zustand verursacht wird $\left(\beta_{193 \mathrm{~nm}}^{*}=-0.00 \pm 0.1\right)$. Das Verzweigungsverhältnis bleibt in diesem Bereich nahezu unverändert bei $R=0.15-0.2$. In diesem Wellenlängenbereich werden bevorzugt $\mathrm{Br}$ gegenüber $\mathrm{Br}^{*}$ Produkten erzeugt.

Die aktuellen theoretischen Betrachtungen gehen auf die Arbeiten aus der Gruppe Pouilly zurück [Pou98, Peo97]. Sie gründen sich auf zeitabhängige und zeitunabhängige quantenmechanische Rechnungen, basierend auf ab initio Potentialflächen. Weitere Experimente wurden von den Gruppen Wittig [Xu88] und Ashfold [Reg99b] durchgeführt.

\section{HI}

Aus der aktuellen theoretischen Arbeit von Le Roy zur Photodissoziation von HI [Roy02], die alle Arbeiten der vergangenen Jahrzehnte berücksichtigt, gehen zwei zentrale Aussagen 
hervor: Man benötigt keine Kopplung, um die unterschiedlichen Wellenfunktionen der Produktkanäle zu erklären. Die zweite Aussage betrifft die beteiligten Zustände (s. Abb. 1.4). An der Photodissoziation von $\mathrm{HI}$ sind die Potentialflächen von vier elektronisch angeregten Zuständen beteiligt $\left(A^{1} \Pi_{1}, a^{3} \Pi_{0^{+}}, a^{3} \Pi_{1}\right.$ (A-Band) und $t^{3} \Sigma_{1}(B$-Band)). Die Anregung der Zustände ist wie bei $\mathrm{HBr}$ auch stark wellenlängenabhängig.

Der Ausgangskanal I wird je nach Anregungswellenlänge von einer Überlagerung der beiden senkrechten Zustände $A^{1} \Pi_{1}$ und $a^{3} \Pi_{1}$ erreicht. Daraus folgt für die Anisotropie $\beta=-1$. Der Kanal des angeregten Zustandes ${ }^{*}{ }^{*}$ wird für große Wellenlängen $(>200 \mathrm{~nm})$ hauptsächlich über die Potentialfläche des $a^{3} \Pi_{0^{+}}$Zustandes bevölkert, der aus einem parallelen Übergang hervorgeht $(\beta=2)$. Zwischen 200 und $180 \mathrm{~nm}$ findet eine immer stärkere Beteiligung des senkrechten Übergangs in den Zustand $t^{3} \Sigma_{1}$ statt, der auch in den Produktkanal I* führt. Die Dominanz dieses Übergangs unterhalb von $180 \mathrm{~nm}$ führt in diesem Bereich zu einem Anistotropiefaktor von $\beta^{*}=-1$. Messungen in diesem Wellenlängenbereich sind noch nicht durchgeführt worden.

Das Verzweigungsverhältnis steigt wellenlängenabhängig von $R_{300 \mathrm{~nm}}=0.1$ auf $R_{250 \mathrm{~nm}}=1.2$ an. Es fällt dann wieder auf $R_{200 \mathrm{~nm}}=0.25$ [Reg99a, Gen98] ab, bevor der $t^{3} \Sigma_{1}$ Zustand die gesamte Photodissoziation dominiert und zu einem Verzweigungsverhältnis von $R_{170 \mathrm{~nm}}=1.3$ [Roy02] führt. Im Gegensatz zu $\mathrm{HBr}$ und $\mathrm{HCl}$ spielt der Kanal $\mathrm{X}\left({ }^{2} P_{1 / 2}\right)$ eine deutlich größere Rolle.

\section{Zusammenfassung}

Die Wasserstoffhalogenide stellen ein Beispiel für nicht-adiabatische Effekte in Photodissoziationsprozessen dar. Für das $\mathrm{HCl}$ Molekül gibt es keine Spin-Bahn Wechselwirkung im Franck-Condon Bereich, allerdings tritt bei großen Kernabständen durch Spin-Bahn Kopplung eine Umverteilung des Photodissoziationsflusses auf. Als Extrem auf der anderen Seite steht HI mit einer starken Spin-Bahn Wechselwirkung im Franck-Condon Bereich, die zur Anregung unterschiedlicher Zustände führt. Hier finden während der Dissoziation keine Kopplungen und Umverteilungen in den Zuständen statt. $\mathrm{HBr}$ als dritte Spezies stellt für den Kanal Br einen Hybriden dar. Bei kurzen Anregungswellenlängen koppelt der ${ }^{1} \Pi_{1} \mathrm{Zu}$ stand bei wachsendem Kernabstand in den ${ }^{3} \Sigma_{0}^{+}$Zustand (wie $\mathrm{HCl}$ ), während bei großen Wellenlängen der Kanal $\mathrm{Br}^{*}$ wie bei der Photodissoziation von $\mathrm{HI}$ direkt über die Anregung in den ${ }^{3} \Pi_{0}^{+}$Zustand besetzt wird. Tabelle $1.1 \mathrm{fa}$ ßt die relevanten Daten aus der Photodissoziation für die Experimente dieser Arbeit zusammen. 


\begin{tabular}{|l|c|cc|c|}
\hline & $\mathrm{HCl}$ & \multicolumn{2}{|c|}{$\mathrm{HBr}$} & $\mathrm{HI}$ \\
& $193 \mathrm{~nm}$ & $193 \mathrm{~nm}$ & $243 \mathrm{~nm}$ & $243 \mathrm{~nm}$ \\
\hline \hline$\beta$ & -1.00 & -0.90 & -0.96 & -1.00 \\
$\beta^{*}$ & -0.94 & 0.00 & 1.96 & 2.00 \\
$R$ & 0.69 & 0.18 & 0.20 & 0.93 \\
Ref. & {$[$ Zha97] } & \multicolumn{2}{|c|}{$[$ Bau99b] } & [Roy02] \\
\hline
\end{tabular}

Tabelle 1.1: Die Anisotropiefaktoren für die Kanäle $X(\beta)$ und $X^{*}\left(\beta^{*}\right)$ sowie die Verzweigungsverhältnisse $R=\left[\mathrm{X}^{*}\right] /[\mathrm{X}]$ für $\mathrm{HX}(\mathrm{X}=\mathrm{Cl}, \mathrm{Br}, \mathrm{I})$ bei den in diesen Experimenten verwendeten Wellenlängen.

Dieses Gebiet ist trotz intensiven Studiums in den letzten Jahrzehnten immer noch in Bewegung, was auf der experimentellen Seite z. B. an Lasern mit kürzeren Wellenlängen, höheren Intensitäten und kürzeren Pulsen festzumachen ist, auf theoretischer Seite an besseren Potentialen und größerer Rechenleistung. Das Augenmerk hat sich in letzter Zeit mehr zum Photodissoziationsprozeß der Wasserstoffhalogenide in Umgebungen wie reinen Clustern, Mischclustern und Matrizen verschoben. Bei diesen Betrachtungen kommt dem Käfigeffekt eine zentrale Rolle zu.

\subsubsection{Käfigeffekt}

Die im letzten Abschnitt beschriebene Photodissoziation gilt nur für freie Moleküle, deren Fragmenttrajektorien nach der Dissoziation nicht durch andere Teilchen gestört werden. Dies ist in Umgebungen wie Hochdruckgasen und der kondensierten Phase (Flüssigkeit, Matrix) nicht mehr erfüllt. Die Wechselwirkung der Dissoziationsfragmente mit Umgebungsatomen und -molekülen durch Stöße führt zum Verlust von kinetischer Energie und der Ursprungsrichtung des Geschwindigkeitsvektors [Sch87, Zew92]. Dieses Phänomen nennt man Käfigeffekt. Energie- und Winkelverteilungen von Wasserstofffragmenten sind die zentralen Messgrößen dieser Arbeit. Sie erlauben Aussagen über den Ablauf von Photodissoziationen in Clusterumgebungen. Die Wechselwirkung der Dissoziationsfragmente tritt in diesem Fall mit den Atomen/Molekülen des Clusters auf.

Je höher die Dichte der Umgebung der Photodissoziation ist, desto stärker ist die Wechselwirkung der Dissoziationsfragmente mit dieser Umgebung. Die ersten Beobachtungen und Voraussagen des Käfigeffekts gehen auf Dissoziationen in Flüssigkeiten und Festkörpern zurück [Fra34]. In diesen kommt es zur Ausbildung käfigähnlicher Struktu- 
ren, die auch zur Namensgebung führten. Die Definition dieses Käfigs beinhaltet dabei ausschließlich die erste Solventschale in der Umgebung.

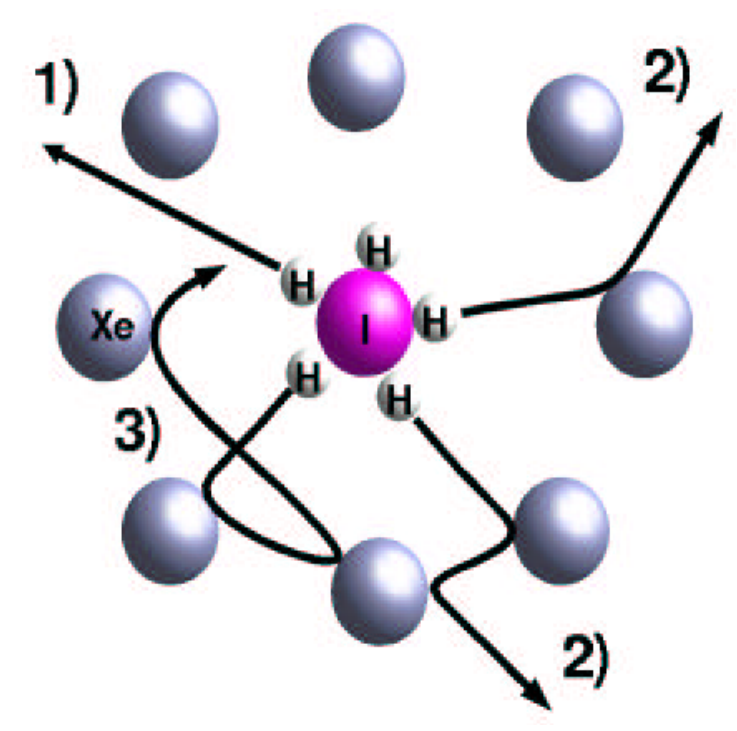

Abbildung 1.5: Schematische Darstellung des Käfig-Effekts am Beispiel der Photodissoziation von $\mathrm{HI}$ in einem Xe Cluster. (1): Direkter Austritt des H Fragments nach der Dissoziation aus dem Cluster. (2): Verzögerter Austritt: Es finden einige Stöße mit den Käfig-Atomen (Xe) statt. (3): Unterbundener Austritt: Das Wasserstofffragment verliert durch Stöße mit den Käfig-Atomen seine gesamte kinetische Energie.

Die möglichen Trajektorien und ihre Klassifizierung aus der Photodissoziation in MatrixExperimenten [Apk99] soll hier erläutert werden. Dabei spielt die Dauer der Wechselwirkung mit dem Käfig eine zentrale Rolle.

Direkter Austritt: (engl. direct exit) In diesem Fall verläßt das Fragment den Käfig ohne relevante Wechselwirkung. Dies kann durch Fragmente von geringer Größe, hohe Fragmentgeschwindigkeiten oder eine günstige Ausrichtung des Geschwindigkeitsvektors auf die Öffnungen des Käfigs, also den Zwischenräumen der Käfigatome untereinander, begünstigt werden [Žd'á99]. Die Fragmente besitzen nach dem Verlassen des Käfigs weitgehend ihre ursprünglichen Eigenschaften nach der Dissoziation.

Verzögerter Austritt: (engl. delayed exit) Gelingt es dem Fragment nicht, den Käfig zu verlassen, so kommt es zum teilweisen Energie- und Impulstransfer auf die Käfigatome. Dieser Übertrag kann zur Aufweitung des Käfigs und dem verzögerten Austritt 
des Fragments führen. Hier besitzt das Fragment noch einen Teil seiner kinetischen Energie, aber durch die Impulsveränderung nicht mehr die Information über die Richtung seines Geschwindigkeitsvektors nach der Dissoziation.

Unterbundener Austritt: (engl. perfect caging) Unterbleibt eine Zerstörung oder Aufweitung des Käfigs und damit ein Austritt, so wird der Käfigeffekt vollständig. Die Energie des Fragments wird an den Käfig abgegeben und die Aufenthaltsdauer unterliegt damit keiner Begrenzung. So gelang in Matrixexperimenten der Nachweis von eingefangenen Fragmenten über einen Zeitraum von fünf Minuten [Law88].

Der Käfigeffekt ist in Abb. 1.5 für die Photodissoziation von $\mathrm{HI}$ in einem Xe Cluster skizziert.

Ein weiterer Effekt, der in Clusterumgebungen aber auch in Matrizen und Flüssigkeiten eine Rolle spielt, ist die Rekombination. Dissoziationsfragmente können vom Käfig abprallen und mit dem Partnerfragment rekombinieren. Das Ursprungsmolekül befindet sich nach der Rekombination in einem schwingungsangeregten Zustand. Die Absorption eines weiteren Photons führt zur erneuten Dissoziation des Moleküls [Niv97, Ale88, Pap91, Pap93, Vor97, Liu93, Wan95].

Bei der Clusterpräparation gibt es die Möglichkeit, die Photodissoziation eines einzelnen Moleküls (Chromophor) in einer ansonsten von der Photodissoziation unabhängigen Umgebung zu betrachten, z. B. Halogenwasserstoff in einer Edelgasclusterumgebung. Hier kann Schwingungsanregung nur durch Rekombination auftreten. Es lassen sich aber auch Mischcluster mit mehreren Chromophor-Molekülen oder reine Chromophor-Cluster präparieren. In diesen ist es möglich, daß ein Fragment aus der Dissoziation durch Stöße ein anderes Molekül energetisch anregt (Schwingung, starke Rotation). Dieses kann dann ebenfalls photodissoziiert werden, was sich in der Energieverteilung als Zusatzenergie der Fragmente bemerkbar macht.

Die Energiebilanzgleichung der Photodissoziation (Gl. 1.5) von freien Halogenwasserstoffen verändert sich in einer Clusterumgebung. Durch Rekombinationen und Schwingungsanregungen sind Energieerhöhungen möglich. Wechselwirkungen mit dem Clusterkäfig dagegen führen zum Energieverlust der Wasserstofffragmente.

Der Käfigeffekt im Cluster ist von mehreren äußeren Parametern abhängig, die im Rahmen dieser Arbeit studiert werden. Zuerst ist die Position des Chromophors zu nennen. Ist es auf der Oberfläche adsorbiert, ist es ein oder zwei Lagen in den Cluster eingedrungen oder sitzt es im Zentrum? Mit der Position ist auch die Frage nach der Ausrichtung des 


$$
h \nu+\underbrace{E_{\text {int }}(\mathrm{HX})}_{\text {Gewinn }}-D_{0}=\underbrace{E_{\text {int }}(\mathrm{X})+E_{C l u}}_{\text {Verlust }}+E_{k i n}(\mathrm{X})+E_{k i n}(\mathrm{H})
$$

$\begin{array}{ll}h \nu & \text { Photonenenergie } \\ E_{\text {int }}(\mathrm{HX}) & \text { Energiegewinn durch Schwingungsanregung oder Rekombination } \\ D_{0} & \text { Dissoziationsenergie } \\ E_{\text {int }}(\mathrm{X}) & \text { Spin-Bahn Aufspaltung von } \mathrm{HX} \\ E_{k i n}(\mathrm{X}) & \text { Kinetische Energie des X Fragments } \\ E_{k i n}(\mathrm{H}) & \text { Gemessene kinetische Energie des H Fragments }\end{array}$

Chromophormoleküls verknüpft. Wenn wir die Photodissoziation von HX annehmen: Zeigt das leichte Wasserstoffatom in Richtung des Clusterzentrums oder zeigt es von ihm weg. Diese Orientierung bestimmt, wie groß der Käfig tatsächlich ist, den das Wasserstoffatom sieht. Eine weitere Frage ist der Einfluß der Clustergröße, sprich des Käfigs selbst? Ab welcher Clustergröße gibt es nur noch vollständig abgebremste Wasserstoffatome (unterbundener Austritt)? Ebenfalls ist die Beschaffenheit des Käfigs selbst während und infolge der Photodissoziation zu berücksichtigen. Die Matrix als Festkörper ist im Modell ein System unendlicher Größe. Der Cluster mit endlicher Größe kann durch Energieüberträge der Dissoziationsfragmente Monomere oder größere Teile abdampfen. Dies kann bis zur vollständigen Fragmentierung des Wirtsclusters, d. h. Zerstörung des Käfigs führen.

Diese Fragen sind bereits intensiv theoretisch diskutiert worden. Dabei ist die Pionierarbeit zur Clustergrößenabhängigkeit von Schinke et al. [Sch95] zu nennen und die intensiven Studien der Gruppe von R.B. Gerber in Kooperation mit unserer Gruppe am Modellsystem $\mathrm{HCl}-\mathrm{Ar}_{\mathrm{n}}$ zur Positions-, Richtungs- und Größenabhängigkeit sowie zur Rekombinationsdynamik [Kry97, Niv97, Niv99, Bau00]. Energieverteilungen für die Photodissoziation von $\mathrm{HI}, \mathrm{HBr}$ und $\mathrm{HCl}$ in Edelgasclusterumgebungen werden von Jungwirth et al. berechnet [Žd'á99, Žd'á00, Sla00, Sla01].

Der zentrale Parameter für den Einfluß des Käfigeffekts auf die Energieverteilung der Wasserstofffragmente aus der Photodissoziation von $\mathrm{HX}$ in einer Edelgasclusterumgebung ist die Anzahl der Schalen, die das Wasserstofffragment bis zum Austritt durchqueren müßte. Dabei sieht ein Wasserstofffragment von der Oberfläche eines Clusters je nach Orientierung mehr Schalen, als eines aus dem Zentrum des Clusters. Ersteres muß eventuell den kompletten Cluster, letzteres nur den halben durchqueren.

Eine weitere Thematik, die mit dem Käfigeffekt verbunden ist, ist die Komplexbildung 
von neuen Molekülen, die der Photodissoziation in Matrizen und Edelgasen folgt. Bei der Photodissoziation von Chromophoren in Edelgasumgebungen werden $\mathrm{X}-\mathrm{Rg}_{\mathrm{n}}$ Moleküle wie z. B. $\mathrm{Xe}_{2} \mathrm{H}$ [Pet99] oder Ar-Cl [JM00] gebildet. Eine prominente Spezies aus der Photodissoziation von $\mathrm{HX}(\mathrm{X}=\mathrm{F}, \mathrm{Cl}, \mathrm{Br}, \mathrm{I})$ in Edelgasumgebungen wird in Abschnitt 1.4 behandelt [Pet95, Bau01b].

Einen ausführlichen Überblick über den Käfigeffekt in Clustern erhält man in [Bau99a]. Allgemein auf photochemische Reaktionen in Clustern geht [Ger94a] ein.

\subsection{Clustererzeugung}

Für Photodissoziationsexperimente von Halogenwasserstoffen in Edelgasclusterumgebungen werden für diese Experimente Cluster mittels Düsenstrahlexpansion eines Edelgases ins Vakuum erzeugt. Dabei setzt unter bestimmten Bedingungen die Bildung von Clustern ein, wobei deren Größenverteilung durch experimentelle Parameter gesteuert werden kann. Die Edelgaswirtscluster werden in einer Koexpansion mit Halogenwasserstoff oder in einem Pick-up Prozeß mit diesen dotiert.

\subsubsection{Düsenstrahlexpansion}

Wenn die mittlere freie Weglänge $\lambda$ eines Gases sehr viel kleiner als die Düsenöffnung $d$ der Strahlquelle ist, so gilt für die charakteristische Größe der Expansion, die Knudsenzahl

$$
K_{n}=\frac{\lambda}{d} \ll 1 .
$$

In diesem Fall kann mit der unter hohem Druck stattfindenden Expansion des Gases ins Vakuum ein Düsenstrahl erzeugt werden. Dieser zeichnet sich gegenüber effusiven Strahlen $\left(K_{n}>1\right)$ vor allem durch eine um drei Größenordnungen höhere Intensität und eine sehr viel schmalere Geschwindigkeitsverteilung aus. Die bei einer idealen Expansion maximal erreichbare Strömungsgeschwindigkeit $u$ dieses Düsenstrahls kann aufgrund der stattfindenden Umwandlung der Enthalpie des Gases in der Quelle in kinetische Energie der Strömung berechnet werden:

$$
u=\sqrt{\frac{2 k T_{0}}{m} \cdot \frac{\gamma}{\gamma-1}},
$$




$$
\begin{array}{ll}
\text { mit } \quad k & : \text { Boltzmannkonstante } \\
T_{0} & : \text { Temperatur der Quelle } \\
m & : \text { Teilchenmasse } \\
\gamma & : \text { Adiabatenexponent } c_{p} / c_{v} .
\end{array}
$$

Die Relaxation wird durch die abnehmende Wahrscheinlichkeit für Teilchenstöße gestoppt, und es verbleibt eine thermische Restgeschwindigkeit $\alpha$, die sich der Strahlgeschwindigkeit überlagert.

$$
\alpha=\sqrt{\frac{2 k T_{\text {Rest }}}{m}}
$$

mit $T_{\text {Rest }}$ : Resttemperatur aus der Relaxation.

Die resultierende Geschwindigkeitsverteilung des Strahls ist eine der Strömungsgeschwindigkeit $u$ überlagerte Maxwellverteilung:

$$
f(v) d v \propto \frac{v^{2}}{\alpha^{3}} \cdot \exp \left[-\frac{(u-v)^{2}}{\alpha^{2}}\right] d v .
$$

Das Maximum der Verteilung, die wahrscheinlichste Geschwindigkeit $v_{w}$ liegt bei

$$
v_{w}=\frac{u}{2}\left[1+\sqrt{1+4\left(\frac{\alpha}{u}\right)^{2}}\right] .
$$

Das Geschwingkeitsverhältnis $S$ (speed ratio)

$$
S:=\frac{u}{\alpha}
$$

gibt dabei das Verhältnis der Strömungsgeschwindigkeit $u$ zur thermischen Geschwindigkeit $\alpha$ an. Für große Werte des Geschwindigkeitsverhältnisses $(S>10)$ ist die Strömungsgeschwindigkeit $u$ gleich dem Maximum dieser Verteilung. In diesem Fall läßt sich $S$ näherungsweise aus der Breite und dem Maximum der Verteilung angeben:

$$
S=2 \sqrt{\ln 2 \frac{v_{w}}{\Delta v}} \approx 1.66 \frac{v_{w}}{\Delta v} .
$$

Das speed ratio ist ein Maß für die Strahlgüte. Mit Ar Strahlen erreicht man in dieser Apparatur typischerweise $S \geq 20$. Für eine eingehendere Beschreibung der Düsenstrahlen verweise ich auf den Übersichtsartikel von D.R. Miller [Mil88]. 


\subsubsection{Clusterbildung und Größenverteilung}

Kommt es in der Expansion eines Überschalldüsenstrahls zur Übersättigung und Kondensation der Teilchen, wird von Clusterbildung gesprochen. Da dieser durch Stöße verursachte Prozeß statistischer Natur ist, bildet sich eine Clusterverteilung, die sich ab einer charakteristischen mittleren Größe $\bar{N}$ mathematisch durch Log-Normalverteilungen beschreiben läßt [Ste01, Sch96, Póc91]. Dieser Grenzwert für $\bar{N}$ beschreibt einen Wechsel im Clusterbildungsprozeß, ab dem die Bildung durch Anlagerung von Monomeren an vorhandene Cluster durch Cluster-Cluster-Stöße, der Koagulation, abgelöst wird.

Die Manipulation dieser Größenverteilungen ist nur über die Variation der Expansionsbedingungen wie Quellentemperatur, -druck und Düsendurchmesser möglich. Eine Beschreibung der Abhängigkeiten und eine zusammenfassende Charakterisierung der Quellenbedingungen wird von Hagena durch ein Skalierungsgesetz für die Erzeugung von Edelgasclustern vorgenommen [Hag81]. Im Parameter $\Gamma$ werden dazu die Quellenparameter in folgender Form vereinigt:

$$
\Gamma=n_{0} d_{0}^{q} T_{0}^{(0.25 q-1.5)} \quad \text { mit } \quad 0.5<q \leq 1
$$

mit $\quad n_{0} \quad$ : Teilchendichte in der Quelle

$d_{0} \quad$ : Durchmesser der verwendeten Lochdüse

$T_{0} \quad$ : Temperatur der Quelle

$q \quad$ : experimentell ermittelter Exponent $(q=0.85$ [Hag92]).

Hagena erweitert seine Theorie um den Parameter $\Gamma^{*}$, der zusätzlich die Gaseigenschaften berücksichtigt, so daß unabhängig von der Gasart die zu erwartende Clustergröße in Abhängigkeit dieses Parameters vorhergesagt werden kann [Hag87]:

$$
\Gamma^{*}=\frac{\Gamma}{\Gamma_{c h}} .
$$

Der Parameter $\Gamma_{c h}$ wird dabei in folgender Weise berechnet:

$$
\Gamma_{c h}=r_{c h}^{q-3} T_{c h}^{0.25 q-1.5} .
$$

Die charakteristischen Größen $r_{c h}$ und $T_{c h}$ werden ihrerseits in folgender Weise bestimmt:

$$
r_{c h}=\left(\frac{m}{\rho}\right)^{1 / 3} \quad ; \quad T_{c h}=\frac{\Delta H_{0}^{0}}{k}
$$


mit $m \quad$ : molekulare Masse der Teilchen im Strahl

$\rho \quad$ : Dichte des Festkörpers

$\Delta H_{0}^{0} \quad$ : Sublimationsenthalpie

$k \quad$ : Boltzmannkonstante.

Neben den Lochdüsen, für die obige Formel gilt, werden sehr häufig - so auch in den hier vorgestellten Experimenten - konische Düsen verwendet. In diesen Düsen wird der Überschalldüsenstrahl nach Austritt aus der Düsenöffnung noch durch einen Konus geführt. Dies führt zu einer stärkeren Richtungsbündelung und durch die erhöhte Zahl an Stößen im Strahl zu verstärkter Clusterbildung. Eine Aufnahme dieser Düsenart in die Beschreibung geschieht durch den äquivalenten Düsendurchmesser $d_{a q}$, der die Expansionbedingungen in einer konischen Düse einer äquivalenten, größeren Lochdüse gegenüberstellt [Hag81].

$$
d_{a q}=0.736 \frac{d^{*}}{\tan \left(\alpha_{D} / 2\right)}
$$

mit $\quad d_{a q} \quad$ : Durchmesser der äquivalenten Lochdüse

$d^{*} \quad$ : kleinster Öffnungsdurchmesser des Konus

$\alpha_{D} / 2 \quad$ : halber Öffnungswinkel des Konus.

Eine einfach zu handhabende Formel zur Bestimmung von $\Gamma^{*}$ entsteht durch die Zusammenfassung der Gleichungen 1.19 und 1.20. Mit der Darstellung in praktikablen Einheiten und $K_{c h}$ als Zusammenfassung der stoffspezifischen Konstanten ergibt sich

$$
\Gamma^{*}=\frac{p_{0}[\mathrm{mbar}] d_{a q}[\mu \mathrm{m}]^{0.85}}{T_{0}[\mathrm{~K}]^{2.2875}} K_{c h} .
$$

Die in dieser Arbeit relevanten Werte für die Skalierung von Edelgasclustern sind in [Sta89] aufgeführt und werden hier in Tabelle 1.2 zusammengefaßt.

\begin{tabular}{|ll|c|c|c|l|}
\hline & & $\mathbf{N e}$ & $\mathbf{A r}$ & $\mathbf{K r}$ & \multicolumn{1}{|c|}{$\mathbf{X e}$} \\
\hline \hline$r_{c h}$ & {$[\mathrm{~nm}]$} & 0.281 & 0.335 & 0.356 & 0.386 \\
\hline$T_{c h}$ & {$[\mathrm{~K}]$} & 230.9 & 939.6 & 1346.1 & 1907.2 \\
\hline$\Gamma_{c h}$ & {$\left[10^{16} \mathrm{~m}^{-2.15} \mathrm{~K}^{-1.2875}\right]$} & 31.07 & 3.495 & 1.931 & 1.036 \\
\hline$K_{c h}$ & {$\left[\mathrm{~K}^{2.2875} \mathrm{mbar}^{-1} \mu \mathrm{m}^{0.85}\right]$} & 185 & 1646 & 2980 & 5554 \\
\hline
\end{tabular}

Tabelle 1.2: Charakteristische Größen der verwendeten Edelgase [Sta89]. 
Mit $\Gamma^{*}$ wird ein Parameter geschaffen, mit dem sich Expansionen unabhängig von der Düsenart oder dem Gas vergleichen lassen. Diese Charakterisierung der Expansion läßt die Frage nach einem festen Zusammenhang mit der Clusterbildung in der Expansion und damit mit der vorliegenden mittleren Clustergröße $\bar{N}$ entstehen. Dieser Zusammenhang ist experimentell in [Kro93, Mül90, Sch96, Hag92] untersucht worden und liefert eine Gleichung der folgenden Form:

$$
\bar{N}=K \cdot\left(\Gamma^{*}\right)^{\zeta} \quad .
$$

Allgemein wird von Hagena eine Klassifizierung für $\Gamma^{*}$ vorgeschlagen:

$$
\begin{array}{rlll} 
& \Gamma^{*}<200 & \text { keine Clusterbildung } \\
200<\Gamma^{*}<1.000 & \text { beginnende Clusterbildung bis zu } \bar{N}=100 \\
1.000<\Gamma^{*} & & \text { massive Clusterbildung }
\end{array}
$$

Auch hier stellt sich die Frage nach dem experimentell wichtigen Parameter, mit dem sich die mittlere Clustergröße $\bar{N}$ effektiv variieren läßt. Wie aus den Betrachtungen zur Maximierung der Strahlintensität deutlich wird, sind zur Erzielung einer maximalen Intensität bei hohem Speed Ratio $S$ bereits die Werte für Expansionsdruck und Düsendurchmesser vorgegeben. Als elegante Möglichkeit bietet sich deshalb die Variation der Düsentemperatur $T_{0}$ an, weil die Temperatur die mittlere Clustergröße mit einer größeren Potenz als die Intensität beeinflußt (s. Gl. 1.24).

Aus dem Parameter $\Gamma^{*}$ läßt sich experimentell die mittlere Clustergröße $\bar{N}$ bestimmen. Dabei werden die von Krohne ermittelten Parameter für Ar Cluster verwendet. Die dort zur Clustergrößenbestimmung benutzte Methode basiert auf der Analyse elastischer Stoßprozesse von Heliumatomen und Clustern und liefert daher wegen der fehlenden Fragmentierung direkt eine Größenangabe nach GI. 1.25 [Buc96]:

$$
\bar{N}=38.4 \cdot\left(\frac{\Gamma^{*}}{1000}\right)^{1.64}
$$

Es ist hier darauf hinzuweisen, daß diese Parameter aus der Untersuchung von Ar Clustern stammen. Daraus ergibt sich die Frage, wie sich die Größenverteilungen für die anderen Edelgase verhalten. Untersuchungen zu Argon, Krypton und Xenon sind in [Sch96, Mül90] zu finden, die eine Übertragung auf diese Edelgase zulassen. Für Neoncluster kann dieses Verhalten ebenfalls gezeigt werden [Kak97, Hag81]. 
Eine Übersicht über Clustererzeugung auch mit anderen Methoden ist in [Hab94] nachzulesen.

\subsubsection{Erzeugung von Mischclustern}

Cluster, die sich aus zwei oder mehreren verschiedenen Bestandteilen zusammensetzen, bezeichnet man als Mischcluster. Dabei gibt es den Spezialfall eines großen Clusters (Wirtscluster), der nur ein oder wenige fremde Atome/Moleküle (Chromophor) enthält. Diese können auf der Oberfläche adsorbiert sein oder in dieser stecken. Es besteht auch die Möglichkeit, daß sie in den Wirtscluster eindringen oder sich in dessen Zentrum befinden. Allgemein spricht man von einem dotierten Cluster. Wenn sich mehrere Fremdteilchen in der Wirtsclusterumgebung befinden, können diese ebenfalls in oder auf diesem zu einem Cluster (Gastcluster) koagulieren.

\section{Koexpansion}

Eine einfache Erzeugung von Mischclustern ist die Mischung der beiden Komponenten und die gemeinsame Expansion durch eine Düse (bevorzugt konisch). Gegebenenfalls verwendet man ein zusätzliches Trägergas, um die Strahleigenschaften zu verbessern [Des94]. Bei der gemeinsamen Expansion (Koexpansion) werden die Fremdatome in der Regel im Inneren des Wirtsclusters eingebaut [Ale88, Pap91]. Sie befinden sich im Zentrum als Kondensationskern für den Wirtscluster.

Die Koexpansion eines Gasgemisches führt zur Veränderung der Strahleigenschaften, so daß man eine mittlere Masse $m_{m}$ der Teilchen im Strahl einführt:

$$
m_{m}=c_{m} \cdot m+\left(1-c_{m}\right) \cdot m_{T}
$$

mit $\quad m_{T} \quad$ : Masse der leichten Trägergasteilchen

$c_{m} \quad$ : Konzentration der schweren Teilchen im Gemisch

$m \quad$ : Masse der schweren Teilchen

Diese neu definierte mittlere Masse $m_{m}$ findet nun Eingang in die Beschreibung der wahrscheinlichsten Geschwindigkeit $v_{w}\left(m_{m}\right)$ des expandierten Gemisches:

$$
v_{w}\left(m_{m}\right)=\sqrt{\frac{m_{T}}{m_{m}}} \cdot v_{w}\left(m_{T}\right)
$$

mit $\quad v_{w}\left(m_{T}\right) \quad$ : wahrscheinlichste Geschwindigkeit des Trägergases 


\section{Teilcheneinfang (Pick up)}

Wenn ein Clusterstrahl eine gasgefüllte Zelle passiert, besteht die Möglichkeit, daß die Cluster ein oder mehrere Gasteilchen einfangen. Liegt die Wechselwirkungszone im Bereich der Expansion des Clusterstrahls, kann die gemeinsame Expansion dazu führen, daß die Fremdmoleküle als Kondensationskeime wirken und im Zentrum der Cluster eingebaut werden. Findet die Wechselwirkung nach Abschluß der Wirtsclusterbildung statt, können die Chromophore auf der Oberfläche angelagert oder in diese eingelagert werden. Wenn der Wirtscluster flüssig ist (Helium, teilweise auch Neon) kann das Chromophor je nach Wechselwirkung zwischen Cluster und Chromophor auch in das Zentrum desselben eindringen $^{6}$. Der Einfang von Molekülen und deren Position im Wirtscluster wird in der Theorie ausführlich von $\mathrm{H}$. Vach behandelt [Vac99, Vac00] und ist experimentell Gegenstand dieser Arbeit.

Der Impulsübertrag des Chromophors auf den Wirtscluster führt aufgrund des Massenverhältnisses zu keiner Ablenkung im Strahl. Die Überschußenergie verteilt sich über den Cluster und kann gegebenfalls zum Abdampfen einiger Clusteratome führen. Ebenfalls ist ein lokales Schmelzen des Wirtsclusters verbunden mit dem Eindringen des Chromophors mit anschließender Rekristallisierung des dann dotierten Clusters möglich [Vac00].

Die beschriebene Methode zur Dotierung von Clustern bzw. zur Erzeugung von Mischclustern wird als Pick-up Verfahren bezeichnet [Gou85]. Ist die Gasdichte der Moleküle genügend groß, so können auch zwei oder mehrere Gastmoleküle vom Wirtscluster aufgenommen werden und selbst größere Cluster auf dem Substratcluster bilden [Hui93].

Die Effektivität des Pick-up Prozesses hängt vom Einfangquerschnitt des Clusters und von der Wechselwirkung der Teilchen, in dieser Arbeit Edelgas mit Halogenwasserstoff, ab. Eine ausführliche mathematische Beschreibung zum Pick-up Prozeß ist in [Bau99a] zu finden. Die Anzahl der eingefangenen Chromophore in Abhängigkeit vom Pick-up Zellendruck gehorcht dabei einer Poisson-Verteilung.

\footnotetext{
${ }^{6}$ Die Wechselwirkung zwischen Alkaliatomen und He Atomen/Clustern ist schwächer als die $\mathrm{He}-\mathrm{He}$ Wechselwirkung. Dies verhindert ein Eindringen in den Cluster. Alkaliatome und -cluster lagern sich deswegen sehr schwach gebunden an die Oberfläche von He Clustern oder werden reflektiert[Hig98].
} 


\subsubsection{Ikosaederstruktur von Edelgasclustern}

In dieser Arbeit wird die Photodissoziation von Molekülen in Edelgasclusterumgebungen studiert. Aus diesem Grund soll hier kurz die Struktur der Edelgascluster diskutiert werden. Bis zu einem bestimmten Größenbereich bilden sie eine Ikosaederstruktur.

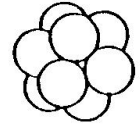

13
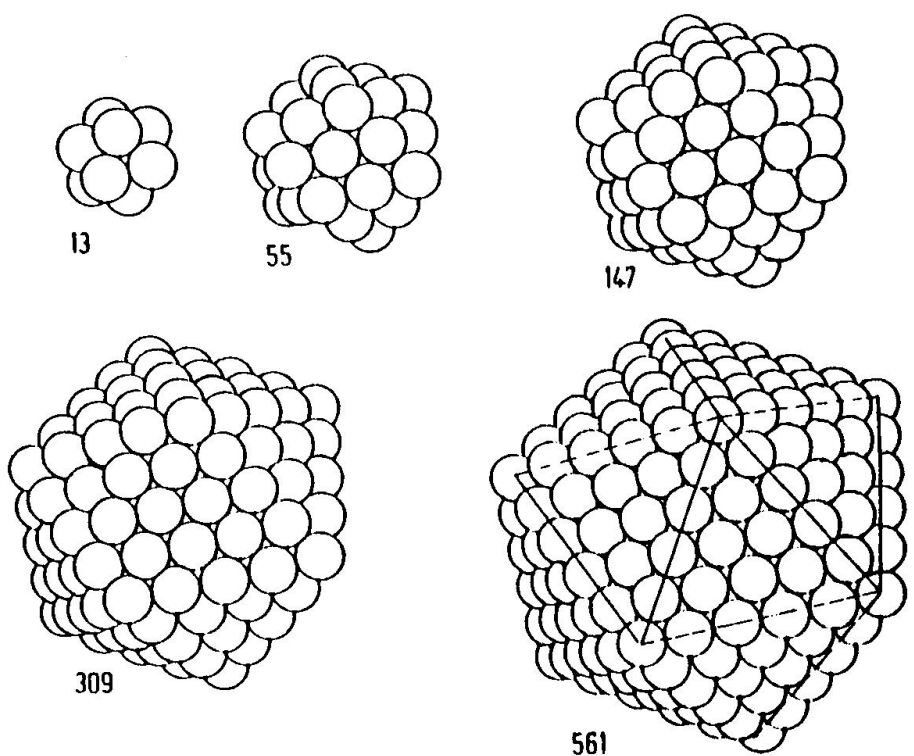

561

Abbildung 1.6: Ikosaeder-Strukturen von Edelgasclustern aus [Hab94]

In der Düsenstrahlexpansion können Edelgascluster im Rahmen der durch die Expansionsparameter bestimmten Verteilung jede Clustergröße einnehmen. Ähnlich wie bei Elektronen- oder Kernkonfigurationen gibt es auch hier sogenannte magische Zahlen, die auf einen Schalenabschluß zurückzuführen sind. Die Cluster bilden wie schon erwähnt eine Ikosaederstruktur aus. Die kleinsten Cluster mit vollen Schalen enthalten $I_{K}=13,55,147$, 309, 561, 923, 1415, 2057 Atome. Die Ikosaederzahl läßt sich nach

$$
I_{K}=\frac{1}{3}\left(10 K^{3}-15 K^{2}+11 K-3\right)
$$

berechnen. Dabei ist allerdings zu berücksichtigen, daß für Ar Cluster mit mehr als 1000 Atomen ein Übergang zum fcc Gitter des Festkörpers postuliert wird. Die Ausprägung der magischen Zahlen nimmt in diesem Bereich ab [Har86]. Ikosaederstrukturen für die ersten fünf magischen Zahlen sind in Abb. 1.6 dargestellt. 


\section{$1.3 \quad$ Flugzeit-Spektroskopie}

In der Flugzeit-Spektroskopie werden lonen in elektrostatischen Feldern richtungsgebunden beschleunigt und je nach Betriebs-Modus aufgrund unterschiedlicher Masse oder kinetischer Anfangsenergie zu verschiedenen Zeiten nachgewiesen. Eine charakteristische Größe eines Flugzeitmassenspektrometers (TOFMS: Time-of-flight mass spectrometer) ist sein Auf|lösungsvermögen, charakterisiert durch das Verhältnis $m / \Delta m=t / 2 \Delta t{ }^{7}$
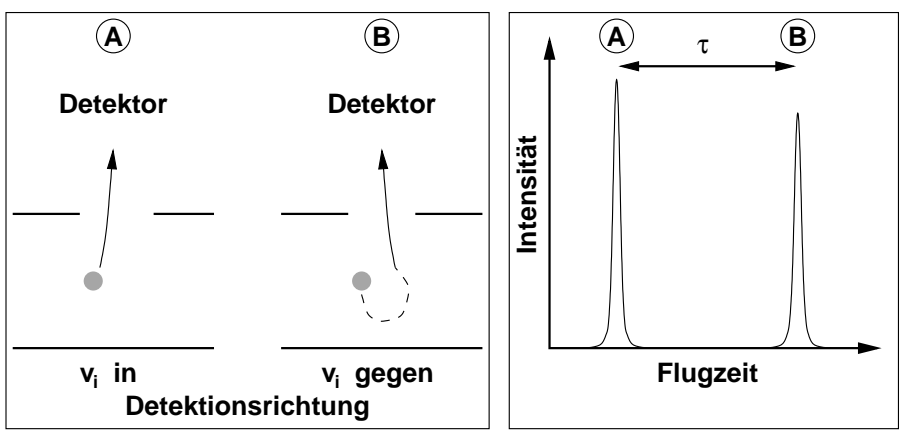

Abbildung 1.7: Einfluß der turn-around-Zeit auf Flugzeitspektren im Niedrigfeldmodus. Ionen mit einer Anfangsgeschwindigkeit entgegengesetzt der Detektionsrichtung (B) werden durch das Abzugsfeld abgebremst und anschließend zum Detektor beschleunigt (links). Im Flugzeitspektrum (rechts) werden sie um die turn-around-Zeit $\tau$ später als lonen mit einer Anfangsgeschwindigkeit in Detektionsrichtung $(A)$ nachgewiesen.

Im Rahmen dieser Arbeit wird der energieaufgelöste Nachweis von Wasserstoffionen in einem Wiley-McLaren Flugzeitmassenspektrometer diskutiert. Die Wasserstoffionen stammen aus der Photodissoziation von Wasserstoffhalogeniden und der anschließenden resonanzverstärkten Mehrphotonenionisation (REMPI) des neutralen Wasserstofffragments.

\subsubsection{Wiley-McLaren Flugzeitmassenspektrometer}

Die Konstruktion des zweistufigen Flugzeitmassenspektrometers nach Wiley und McLaren (WMTOFMS) [Wil55], s. Abb. 1.8, kompensiert einen auf|lösungsbegrenzenden Effekt in den Messungen. Durch Erfüllung der Ortsfokussierungsbedingung erreichen Fragmente mit gleichem Verhältnis $\mathrm{e} / \mathrm{m}$ unabhängig vom Startort im Spektrometer zum gleichen

\footnotetext{
${ }^{7} \mathrm{Im}$ Flugzeitmassenspektrometer gilt die Proportionalität: $t \propto \sqrt{m} . t$ ist die Gesamtflugzeit für ein Teilchen der Masse $\mathrm{m}$ und $\Delta t$ die volle Halbwertsbreite des Peaks.
} 
Zeitpunkt den Detektor. Das Problem eines endlichen lonisationsvolumens spielt somit keine Rolle. Weitere auf|lösungsbegrenzende Effekte sind die endliche lonisierungs- und Nachweiszeit [Fri97] und die Geschwindigkeitsverteilung der Fragmente vor der lonisation.

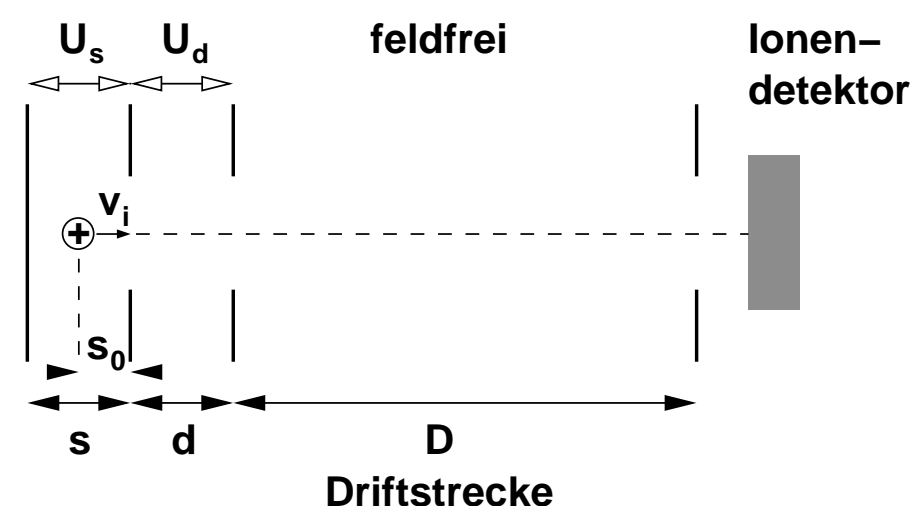

\begin{abstract}
Abbildung 1.8: Zweistufiges Flugzeitmassenspektrometer vom Wiley-McLaren Typ. Die lonen mit ihrer Anfangsgeschwindigkeit $v_{i}$ werden im elektrischen Feld $E_{s}=U_{s} / s$ aus dem lonisierungsvolumen abgezogen. Eine weitere Beschleunigung der Teilchen findet im Feld $E_{d}=U_{d} / d$ statt, bevor die lonen die feldfreie Driftstrecke $D$ durchfliegen und anschließend am lonendetektor nachgewiesen werden.
\end{abstract}

Wie schon in Abschnitt 1.1.3 beschrieben können Fragmente aus der Photodissoziation in genau entgegengesetzte Richtungen fliegen. Wenn man das auf die Detektorachse in einem WMTOFMS bezieht, heißt das, sie haben eine Geschwindigkeitskomponente in oder entgegengesetzt der Detektionsrichtung. Dies führt dazu, daß Fragment-lonen mit gleicher kinetischer Energie aber einer Geschwindigkeitskomponente $v_{i}=-v_{i}$ zu unterschiedlichen Zeiten am Detektor registriert werden. Die Zeitdifferenz bezeichnet man als turn-aroundZeit $\tau$ (s. Gl. 1.36). Dieser Effekt und seine Auswirkung auf das Flugzeitspektrum sind in Abb. 1.7 illustriert. Wenn man die quadratische Korrelation zwischen Zeit- und Energieraum berücksichtigt, wird ein lon gleicher Masse ohne Anfangsgeschwindigkeit genau zwischen den beiden oben genannten lonen nachgewiesen. Je nach Wahl der Betriebsparameter kann man mit dem WMTOFMS somit eine hohe Energieauf|lösung erreichen. Dies ermöglicht die Ermittlung der Energieverteilung eines Fragment-lons (s. Abschnitt 3.2). Der dafür geeignete Betriebsmodus ist der sog. Niedrigfeldmodus, in dem die Energien der elektrostatischen Abzugs- und Beschleunigungsfelder für ein einfach geladenes Teilchen in der Größenordnung der maximalen kinetischen Anfangsenergie der Fragment-lonen liegen.

In der ursprünglichen Anwendung von Flugzeitmassenspektrometern, bei der man mas- 
sensensitiv arbeitet, versucht man, die Massenauf|lösungsbegrenzung durch die Geschwindigkeitsverteilung der lonen mit möglichst hohen elektrostatischen Feldern zu kompensieren.

Ein geladenes Teilchen mit der Anfangsgeschwindigkeit $v_{i}$ und der Ladung $q$ vergröBert im WMTOFMS bei Durchquerung der elektrischen Felder $E_{s}$ (Abzugsfeld) und $E_{d}$ (Beschleunigungsfeld) seine Energie auf

$$
E_{\text {ges }}=\frac{1}{2} m v_{i}^{2}+q U_{s}+q U_{d} \quad .
$$

Auf der Driftstrecke wirken keine äußeren Felder und die Energie bleibt konstant. Damit ergibt sich mit den geometrischen Parametern aus Abb. 1.8 für die Flugzeit des lons vom lonisierungsort $s_{0}$ bis zum Detektor

$$
t_{\text {ges }}\left(s_{0}, v_{i}\right)=t_{s}+t_{d}+t_{D}
$$

wobei die Indizes die Gesamtflugzeit sowie die Flugzeiten im Abzugsfeld (s), Beschleunigungsfeld (d) und in der freien Driftstrecke (D) bezeichnen. Sie ergeben sich im Einzelnen zu:

$$
\begin{aligned}
t_{s} & =\sqrt{\frac{2 m}{q}} \cdot \frac{s}{U_{s}} \cdot\left(\sqrt{U_{0 s}} \pm \sqrt{U_{i}}\right) \\
t_{d} & =\sqrt{\frac{2 m}{q}} \cdot \frac{d}{U_{d}} \cdot\left(\sqrt{U_{0}}-\sqrt{U_{0 s}}\right) \\
t_{D} & =\sqrt{\frac{2 m}{q}} \cdot \frac{D}{2 \sqrt{U_{0}}}
\end{aligned}
$$

mit

$$
\begin{aligned}
k & =s_{0} / s & & \text { Startortparameter der lonen } \\
E_{k i n}^{i}=q U_{i} & =\frac{1}{2} m v_{i}^{2} & & \text { kin. Anfangsenergie der lonen am Ort } s_{0} \\
E_{k i n}^{s}=q U_{0 s} & =q\left(U_{i}+k U_{s}\right) & & \text { kin. Energie am Ende des Abzugsfeldes } \\
E_{\text {kin }}^{g e s}=q U_{0} & =q\left(U_{0 s}+U_{d}\right) & & \text { gesamte kinetische Energie der lonen. }
\end{aligned}
$$

Die räumliche Fokussierung $m$-ter Ordnung von lonen gleicher Masse, aber unterschiedlichen Startparametern läßt sich als

$$
\frac{\partial^{m} t(k)}{\partial k^{m}}=0, \text { wobei } m=1,2,3, \ldots
$$


schreiben. Unter dem Ortsfokus erster Ordnung versteht man den Punkt in der feldfreien Driftstrecke, an dem die lonen mit Anfangsgeschwindigkeit entgegengesetzt zum Detektor diejenigen mit einer Komponente zum Detektor eingeholt haben. Wenn man den Detektor an dieser Stelle platziert, findet keine Begrenzung des Auf|lösungsvermögens durch den Startort der lonen, sprich die Größe des lonisationsvolumen, statt. Typischerweise ist eine Geometrie des WMTOFMS gegeben und man wählt die Felder entsprechend.

Eine nähere mathematische Beschreibung zum WMTOFMS und speziell zur Ortsfokussierung findet sich in [Fri97]. Eine Möglichkeit, deutlich bessere Massenauf||ösungen zu erzielen, ist die Verwendung eines Reflektron Flugzeitmassenspektrometers [Kar72, Boe92], wie es in anderen Experimenten unserer Gruppe zur Bestimmung von Clustergrößenverteilungen verwendet wird [Ste01, Sch96, Hob94].

\section{Niedrigfeldmodus}

In Gl. 1.32 steht $\pm \sqrt{U_{i}}$ für die Anfangsgeschwindigkeit $v_{i}$ in oder entgegengesetzt zum Detektor. Daraus ergibt sich für die Differenz der beiden Fälle die turn-around-Zeit $\tau$ zu

$$
\tau=2 \cdot \frac{m v_{i} s}{q U_{s}}
$$

Diese Verbreiterung des Massenpeaks durch eine Verteilung der Anfangsgeschwindigkeit von lonen gleicher Masse macht man sich bei energieaufgelösten Messungen zunutze. Dabei wird die Meßgenauigkeit der Geschwindigkeit $v_{i}$ mit Absenkung des elektrischen Feldes $E_{s}=U_{s} / s$ weiter erhöht. Wenn man alle lonen unabhängig von ihrer Anfangsflugrichtung in Detektorrichtung nachweisen will, muß das Feld so gewählt werden, daß die lonen mit Anfangsgeschwindigkeit entgegengesetzt dem Detektor noch vor der untersten Platte im WMTOFMS abgebremst werden:

$$
q U_{s} \cdot \frac{s-s_{0}}{s}<\frac{1}{2} m v_{i}^{2} .
$$

Bei kinetischen Energien der ionisierten Fragmente aus der Photodissoziation von einigen Elektronenvolt und Plattenabständen im Bereich Zentimeter führt das zu elektrischen Spannungen von 5-50 V. Den Betriebsmodus des WMTOFMS mit Spannungen in diesem Bereich bezeichnet man als Niedrigfeldmodus im Gegensatz zum Hochfeldmodus in der Massenspektrometrie, bei dem man Spannungen im Bereich einiger Kilovolt anlegt.

Eine starke Absenkung des elektrischen Abzugsfeldes hat zur Folge, daß lonen mit einer transversalen Geschwindigkeitskomponente $v_{\text {trans }}$ je nach deren Höhe, das WMTOFMS 
seitlich verlassen und damit nicht am Detektor nachgewiesen werden.

$$
v_{\text {trans }}>\delta_{r}=\frac{r_{D}}{t_{\text {ges }}}
$$

mit $\quad v_{\text {trans }}:$ Transversale Anfangsgeschwindigkeit des lons

$\delta_{r} \quad$ : maximale transversale Geschwindigkeit, um die

Detektorfläche zu erreichen

$r_{D} \quad$ : Detektorradius

$t_{\text {ges }}$ : Gesamtflugzeit der lonen nach GI. 1.31

gibt an, ab welcher Geschwindigkeit die lonen, das WMTOFMS verlassen [Sya96]. Wenn schon eine geringe Geschwindigkeit $v_{\text {trans }}$ ausreicht, um das lon nicht mehr nachzuweisen, führt dies zum Ausschnitt eines schmalen Zylinders (annähernd eindimensional) mit Verlauf der Achse durch das Zentrum der dreidimensionalen Winkelverteilung der Fragmente. Die Erfüllung dieser Bedingung mit

$$
v_{\text {trans }} \gg \delta_{r}
$$

bezeichnet man als core sampling oder core extraction.

\subsubsection{REMPI}

In diesen Experimenten werden durch den Photodissoziationsprozess neutrale Fragmente erzeugt. Um diese in den Feldern des Wiley-McLaren Flugzeitmassenspektrometers abziehen zu können, müssen sie zuerst ionisiert werden. Dafür bieten sich prinzipiell zwei Möglichkeiten an:

- Elektronenstoßionisation

- Photoionisation

Die Elektronenstoßionisation bietet den Vorteil, daß man mit sehr hohen Elektronenenergien arbeiten kann, und die Absorption eines einzelnen Elektrons genügt, um ein Atom zu ionisieren. Bei niedrigen Elektronenergien kann man die Energien mehrerer Elektronen im Atom dissipieren, bis die lonisationsschwelle erreicht ist. Dies setzt nur voraus, daß die Energie pro Elektron mindestens so groß ist, wie das niedrigste elektronische Anregungsniveau des Atoms (Franck-Hertz-Versuch). 


\begin{tabular}{|l|c|c|c|l|}
\hline $\begin{array}{l}\text { Fragment } \\
\text { Molekül }\end{array}$ & $\begin{array}{c}\text { lonisierungs- } \\
\text { energie [eV] }\end{array}$ & $\begin{array}{c}\text { lonisierung } \\
\mathrm{m}+\mathrm{n}\end{array}$ & $\begin{array}{c}\text { Wellenlänge } \\
{[\mathrm{nm}]}\end{array}$ & Referenz \\
\hline \hline $\mathrm{H}$ & 13.6 & 1 & 91.2 & 1 \\
& & $3+1$ & 364.71 & 3 \\
& & $2+1$ & 243.14 & 4 \\
& & $2+1$ & 205.1 & $5,6,7$ \\
$\mathrm{Cl}$ & 12.97 & $1+1$ & 121.56 & 8 \\
& & $2+1$ & $\approx 210$ & 2 \\
$\mathrm{Cl} *$ & 12.86 & 1 & 96.4 & 1,9 \\
& & $2+1$ & $\approx 241$ & 2 \\
$\mathrm{Br}$ & 11.81 & 1 & 105.02 & 1 \\
& & $2+1$ & $\approx 263$ & 2 \\
$\mathrm{Br} *$ & 11.35 & 1 & 109.3 & 1,2 \\
& & $2+1$ & $\approx 254$ & 2 \\
$\mathrm{I}$ & 10.45 & 1 & 118.7 & 1 \\
& & $2+1$ & 273.77 & 2 \\
$\mathrm{I*}$ & 9.51 & 1 & 130.4 & 1,13 \\
& & $2+1$ & 242.65 & 2 \\
$\mathrm{HCl}$ & 12.75 & 1 & 97.3 & 1 \\
& & $2+1$ & 240 & 10 \\
$\mathrm{HBr}$ & 11.66 & 1 & 106.4 & 1 \\
& & $2+1$ & 269.26 & 11 \\
$\mathrm{HI}$ & \multirow{2}{*}{10.39} & 1 & 119.4 & 1 \\
& & $2+1$ & $280.3-309.4$ & 12 \\
\hline
\end{tabular}

Tabelle 1.3: lonisierungsenergien und -wellenlängen der in diesen Experimenten relevanten Atome und Moleküle. (1): [Lid96], (2): [Moo52], (3): [Har94], (4): [Fri97], (5): [Boo96], (6): [Bet57], (7): [Bau99b], (8): [Nah98], (9): [Liu99], (10): [OE97], (11): [Gre97], (12): [Wri94], (13): [Roy02].

Nachteile der Elektronenstoßionisation sind ein relativ großes lonisationsvolumen und die mangelnde Selektivität gegenüber unterschiedlichen Atomen und Molekülen.

Diese Nachteile erlauben keine Verwendung der Elektronenstoßionisation in diesen Experimenten. Ein zu großes lonisierungsvolumen würde zu einer Unschärfe des Startortes im WMTOFMS führen. Die mangelnde Selektivität würde sowohl die Halogenwasserstoffe als auch die Edelgasatome direkt ionisieren und zu einer Fragmentierung des Moleküls führen (s. Abschnitt 2.1.3). 
Die Photoionisation läßt sich heute mit gepulsten, fokussierten Laserstrahlen realisieren. Die verfügbaren Laserwellenlängen genügen bei den meisten Atomen allerdings nicht, um durch Absorption eines einzelnen Photons das lonisationskontinuum zu erreichen (Tabelle 1.3).

Bei der Absorption mehrerer Photonen sind die beiden Fälle der resonanzverstärkten und der nicht-resonanten Mehrphotonenionisation (MPI: multi photon ionisation) zu unterscheiden. Die Absorption mehrerer Photonen zum Erreichen des lonisationskontinuums oder eines Zwischenniveaus setzt eine hohe Photonendichte voraus (Lichtintensität: $10^{6} \mathrm{~W} / \mathrm{cm}^{2}$ [Let87]), weil die Photonen gleichzeitig absorbiert werden müssen.

Aus zeitabhängiger Störungsrechnung erhält man für die Übergangswahrscheinlichkeit $W_{n}$ einer nichtresonanten $n$-Photonenabsorption [Lin84]

$$
W_{n}=\sigma_{n} I^{n} \tau,
$$

mit dem Photonenfluß I, dem Absorptionsquerschnitt $n$-ter Ordnung $\sigma_{n}$ und $\tau$ der Pulsdauer des Lasers.

Wenn nicht alle Photonen simultan absorbiert werden müssen, steigt die lonisierungswahrscheinlichkeit um mehrere Größenordnungen [Joh81]. Dies läßt sich erreichen, wenn man einen angeregten Zustand des Atoms oder Moleküls zur lonisation ausnutzt. Durch Absorption von $m$ Photonen findet eine Anregung in ein reales Niveau statt. Dieses wird als resonanter Zwischenzustand bezeichnet. Innerhalb seiner Lebensdauer müssen weitere $n$ Photonen zur lonisation aufgenommen werden. Dieser Prozeß führt zu einer resonanzähnlichen Erhöhung der lonisierungswahrscheinlichkeit und wird als resonanzverstärkte Mehrphotonenionisation, REMPI (resonance enhanced multi photon ionisation) bezeichnet. Die mathematische Beschreibung erfolgt über Ratengleichungen, wobei Prozesse wie Fluoreszenz, Prädissoziation und innere Konversion zu berücksichtigen sind, die zu einer Entleerung des resonanten Zwischenniveaus führen. Die Anregung von atomaren Niveaus setzt schmalbandige durchstimmbare Laser voraus.

In Abb. 1.9 sind verschiedene Prozesse zur Photoionisation von Wasserstoffatomen aufgeführt. Die Wellenlänge von $91.2 \mathrm{~nm}$ zur Einphotonenionisation läßt sich durch nichtlineare Frequenzkonversionen höherer Ordnung an einem Molekularstrahl oder durch eine Gasentladung im Vakuum erzeugen. Eine Filterung von Licht unterschiedlicher Wellenlängen ist in diesem Bereich ausschließlich mit reflektiven Optiken erreichbar (VUVMonochromator [Fou91] oder Spiegel unter streifendem Einfall [Sof87]). Bisher gibt es keine transmittiven Materialien unterhalb einer Wellenlänge von $105 \mathrm{~nm}$ [Nah98]. Der 1+1 
REMPI Prozeß, unter Ausnutzung des $2 p$ Niveaus im Wasserstoffatom mit einer Anregungswellenlänge von $121 \mathrm{~nm}$, kam in früheren Experimenten an unserer Apparatur zur Anwendung [Nah98, Bau99b]. Das Licht wurde in einer Edelgasverdreifachungszelle mit einem Gemisch aus Krypton und Argon erzeugt [Mah79, Hil81]. Der experimentelle Aufwand ist deutlich höher als der für den in diesen Experimenten benutzten 2+1 REMPI Prozeß (2s Niveau) mit einer Wellenlänge von $243 \mathrm{~nm}$. Diese UV Strahlung kann mittels Verdopplungs- und Mischungsprozessen in Kristallen erzeugt werden (s. Abschnitt 2.2.1). Für den 1+1 REMPI Prozeß spricht die deutlich höhere Photonenausbeute. In diesen Experimenten ließ sich feststellen, daß man mit einer Laserenergie von $50 \mathrm{~nJ}$ bei $121 \mathrm{~nm}(1+1)$ und einer Laserenergie von $1.6 \mathrm{~mJ}$ bei $243 \mathrm{~nm}(2+1)$ eine ähnliche Ausbeute in der Ionisierung von Wasserstoffatomen erreicht. Eine weitere Möglichkeit der $2+1$ lonisierung mit $205 \mathrm{~nm}$ über das 3d Niveau ist nach einigen Tests nicht weiter eingesetzt worden, weil die kurze Lebensdauer des resonanten Zwischenzustandes einen sehr geringen Querschnitt für diesen Prozeß liefert [Fri97, Nah98, Bau99b, Bau99a].

Die entscheidenden Vorteile der Photoionisation gegenüber der Elektronenstoßionisation werden hier deutlich. Laser lassen sich auf minimal kleine Flächen fokussieren. In diesen Experimenten hat der Fokus der $243 \mathrm{~nm}$ Strahlung einen Durchmesser von weniger als $0.02 \mathrm{~mm}$. Durch die Wahl der Wellenlänge ist die Spezies-Sensitivität in der lonisation der entscheidende Vorteil. Wenn die Wellenlänge nicht ausreicht, andere Atome oder Moleküle direkt zu ionisieren, ist der Querschnitt für diese so gering, daß sie im Massenspektrum nicht als lonen detektiert werden.

\subsection{Eine neue Klasse von Molekülen: Wasserstoff-Edelgas-Halogen (HRgX)}

Edelgase (Rg: rare gas) gehören zu den Atomen mit dem größten Vorkommen im Universum. Lange Zeit haben die Chemiker akzeptiert, daß Edelgase aufgrund der Oktett-Regel (abgeschlossene Elektronenkonfiguration) chemisch nicht reaktiv sind. 1933 postulierte Linus Pauling die Existenz von Verbindungen wie $\mathrm{XeF}_{6}$ und $\mathrm{KrF}_{6}$, die aber erst über dreißig Jahre später erzeugt werden konnten. In den siebziger Jahren des vergangenen Jahrhunderts wurden zahlreiche stabile Verbindungen von Xe mit O, F und $\mathrm{C}$ erzeugt.

Eine neue Bedeutung unter Laborbedingungen bekamen Festkörper der Edelgase in Matrixexperimenten. Dissoziationsmessungen von Wasser, Halogenwasserstoffen und weiteren kleinen Molekülen in Edelgasmatrizen haben zum einen dem Studium des Dissoziations- 


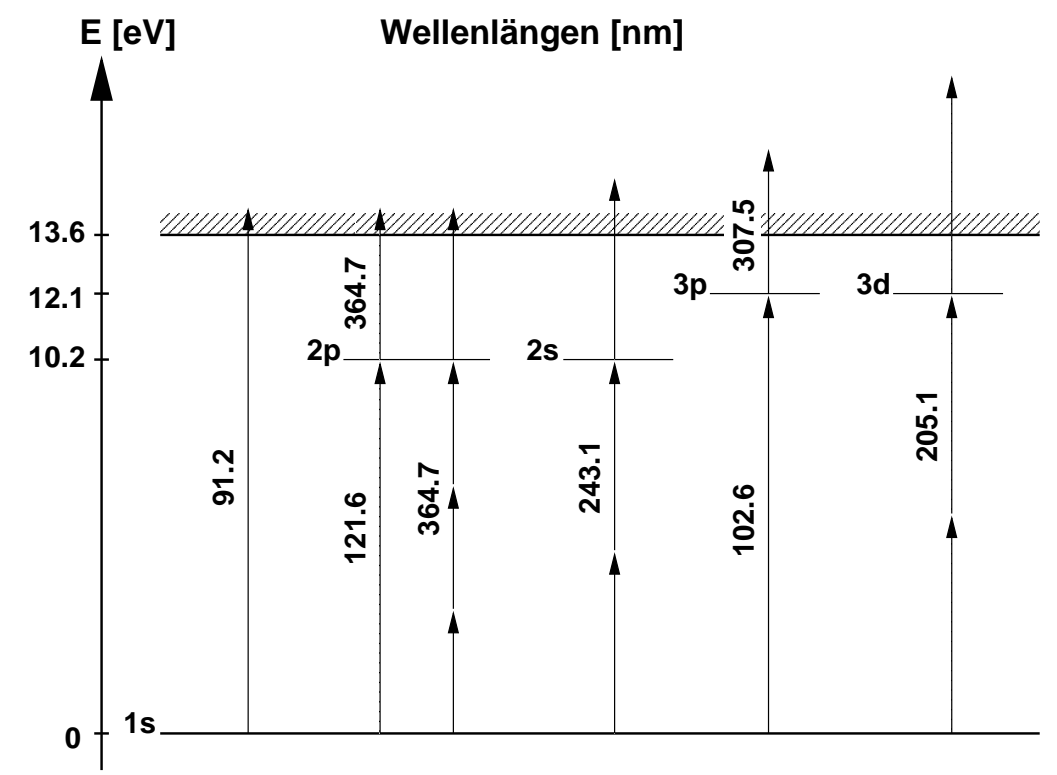

Abbildung 1.9: Möglichkeiten zur Photoionisation von Wasserstoffatomen: Gezeigt sind abgesehen von der Einphotonenionisation (ganz links) mehrere resonanzverstärkte Mehrphotonenionisationen (REMPI) von Wasserstoffatomen. Als resonante Zwischenzustände werden unterschiedliche Niveaus ausgewählt. In diesen Experimenten werden Wasserstoffatome mittels 2+1 REMPI bei einer Wellenlänge von $243 \mathrm{~nm}$ (dritter Prozeß von links) über das $2 \mathrm{~s}$ Niveau ionisiert. Die Werte für die Übergänge sind entnommen aus [Bas75].

prozesses gedient (Dynamik), zum anderen aber auch Erkenntnisse über neue molekulare Verbindungen geliefert, wie $\mathrm{Rg}_{n} \mathrm{H}^{+}$und $\mathrm{X}^{-} \mathrm{Rg}_{n}^{+}(\mathrm{X}=\mathrm{Cl}, \mathrm{Br})$ [Apk99, Law88, Gö96, Tar93, Ger93].

In den neunziger Jahren ist in diesem Zusammenhang eine neue Spezies von Molekülen entdeckt worden: $\mathrm{HRgX}$. Es handelt sich dabei um lineare, polare Moleküle, die sich aus einem Wasserstoffatom $(H)$, einem Edelgasatom $(R g=A r, K r, X e)$ und einem Halogenatom ( $\mathrm{X}=\mathrm{F}, \mathrm{Cl}, \mathrm{Br}, \mathrm{I})$ zusammensetzen. Weitreichende Untersuchungen dieser Komplexe in Edelgasmatrizen betreibt die Gruppe um M. Räsänen. Dabei handelt es sich sowohl um Experimente zur Bildung, Schwingungsanregung und Dissoziation von HRgX [Pet95, Pet99, Khr00, Pet97, Lig02, Kun94, Aho00, Elo99, Lor00] als auch um theoretische Voraussagen und Simulationen zusammen mit der Gruppe von R.B. Gerber [Lun00b, Lun00c, Pet02b, Lun00a, Bih02, Joh99]. Die Existenz dieser Moleküle kann durch 
Infrarotabsorptionsspektroskopie der $\mathrm{Rg}-\mathrm{H}$ Streckschwingung nachgewiesen werden.

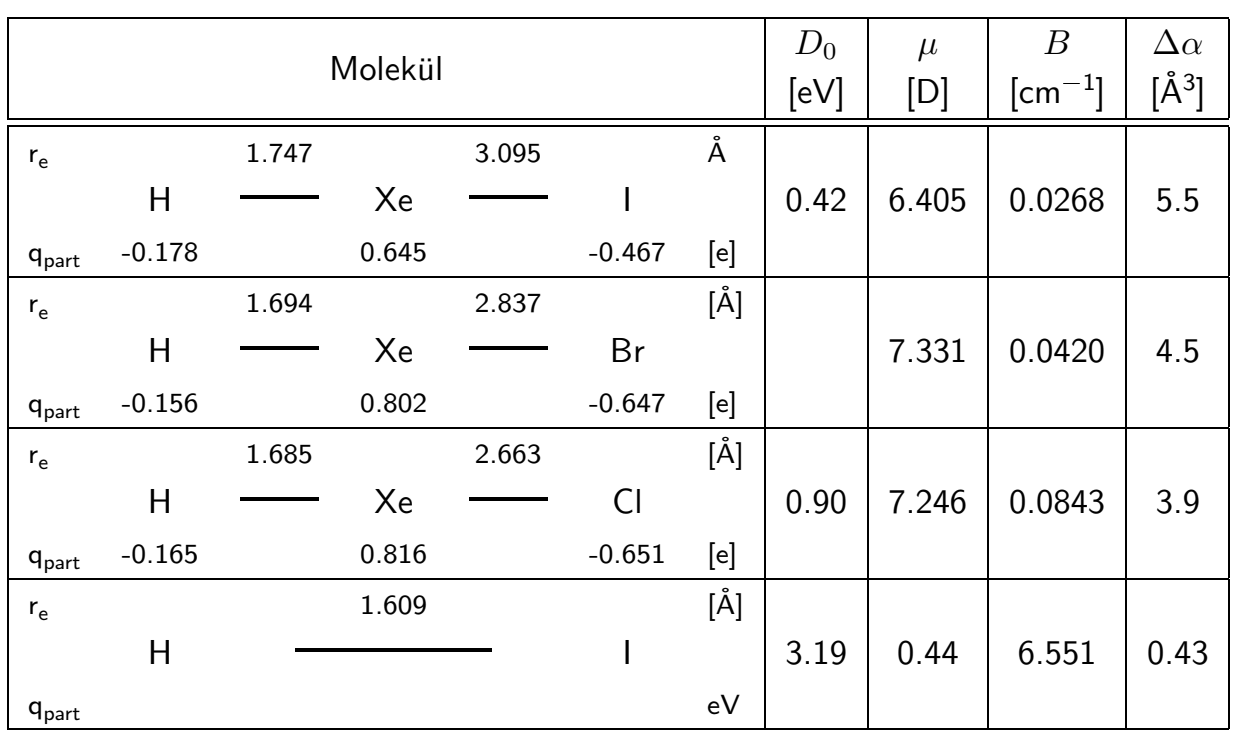

Tabelle 1.4: Moleküldaten von $\mathrm{HXeX}, \mathrm{X}=\mathrm{I}, \mathrm{Br}, \mathrm{Cl}$; und $\mathrm{HI}$ : Dissoziationsenergien $D_{0}$, Dipolmomente $\mu$, Rotationskonstanten $B$, Anisotropie der Polarisierbarkeiten $\Delta \alpha$ nach G. Maroulis [Mar00], Bindungsabstände $r_{e}$ und Partialladungen $q_{\text {part }}$ in Elementarladungen.

Im Rahmen dieser Arbeit wird die Bildung von $\mathrm{HXel}$ und $\mathrm{HXeCl}$ im Anschluß an den Photodissoziationsproze $\mathrm{B}$ von $\mathrm{HI}$ und $\mathrm{HCl}$ in $\mathrm{Xe}_{\mathrm{n}}$ Clustern studiert [Bau01b]. Der Nachweis der nach der Fragmentierung des Clusters freien Moleküle geschieht mittels Orientierung in kombinierten elektrostatischen und Laserfeldern und anschließender Dissoziation durch Potentialkreuzung oder Photonenanregung. Dazu liegen neuere experimentelle und theoretische Arbeiten aus unserer Gruppe in einer Kooperation mit R.B. Gerber vor [Nah02, Coh01]. Die Bildung und Eigenschaften dieser Moleküle und ihre Orientierung in Feldern werden im folgenden behandelt.

\subsubsection{Bildung und Eigenschaften}

Die Photodissoziation eines Wasserstoffhalogenids in einer Edelgasumgebung (Cluster, Matrix) trennt das Halogen- und das Wasserstoffatom. Das Wasserstoffatom, welches die deutlich höhere kinetische Energie besitzt, verläßt die Umgebung des Halogenatoms und bewegt sich in der Edelgasumgebung weiter. 


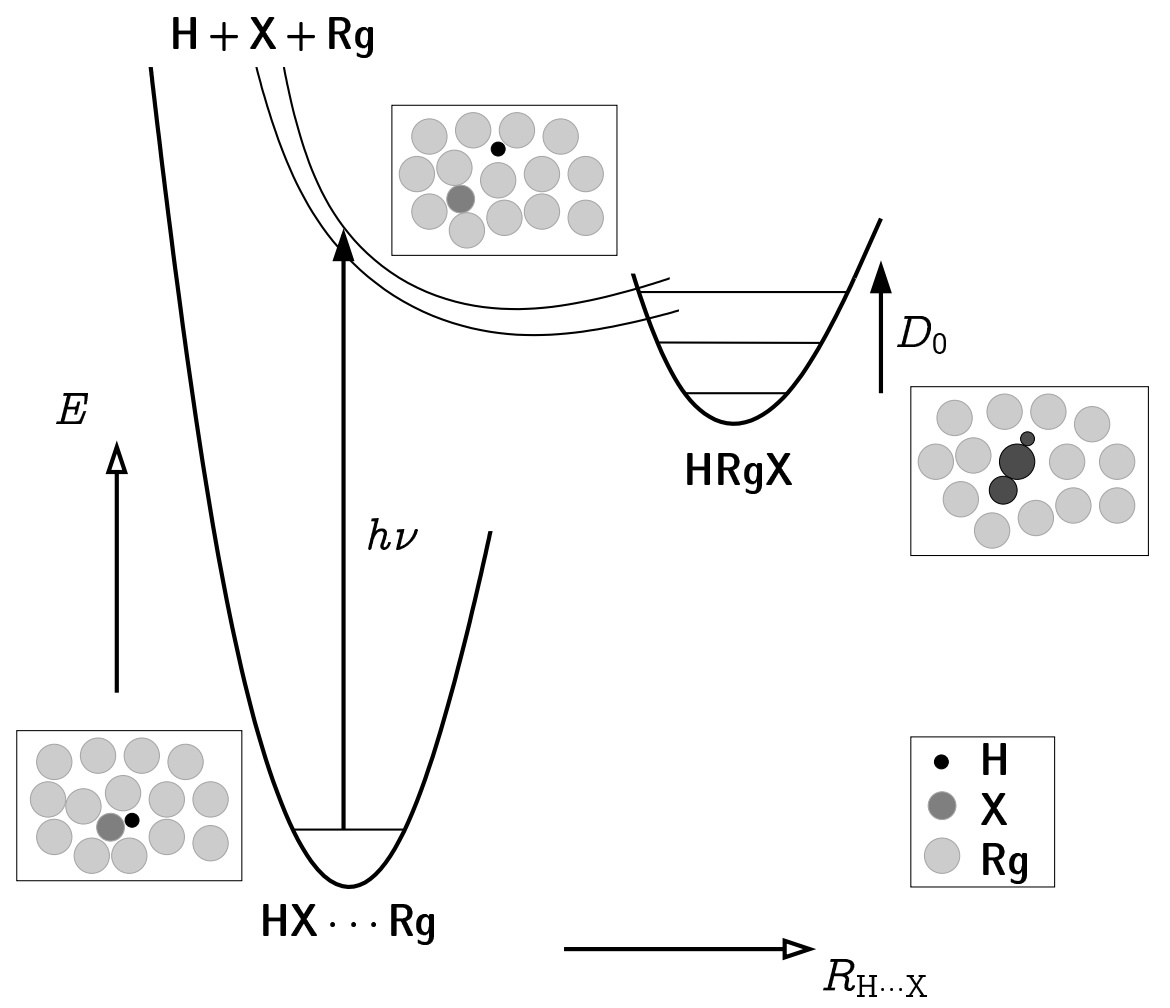

Abbildung 1.10: Bildung von $\mathrm{HRgX}$ in der Photodissoziation von $\mathrm{HX} \cdot \mathrm{Rg}$ : Links unten ist das Potential des gebundenen Zustands von $\mathrm{HX}(\mathrm{X}=\mathrm{F}, \mathrm{Cl}, \mathrm{Br}, \mathrm{I})$ in einer Edelgasumgebung $\mathrm{Rg}$ dargestellt. Im linken oberen Teil sind die durch Photonenanregung erreichten repulsiven Zustände von $\mathrm{HX}$ zu sehen. Die Anwesenheit der Edelgasatome verändert den dissoziativen Kanal mit einem Potentialwall (Mitte) über den die Fragmente $\mathrm{H}+\mathrm{X}+\mathrm{Rg}$ in den gebundenen Zustand $\mathrm{HRgX}$ gelangen können. Die waagrechten Linien symbolisieren Schwingungsniveaus. Die Bindungsenergie $D_{0}$ liegt zwischen 0.4 und $1 \mathrm{eV}$. Die Piktogramme zeigen rechts $\mathrm{HX}$ in $\mathrm{Rg}$, in der Mitte $\mathrm{H}$ und $X$ getrennt nach der Dissoziation in $\mathrm{Rg}$ und links das gebildete $\mathrm{HRgX}$ Molekül in der Rg Umgebung.

Man kann von einer Art cage exit sprechen, wenn man damit den Käfig bezeichnet, den die Edelgasatome ergeben, die dem Halogenatom direkt benachbart sind. Das Wasserstoffatom stößt mit einem weiter entfernten Edelgasatom, von dem es zu einem Edelgasatom des ursprünglichen Käfigs zurückprallt. Dieses Edelgasatom befindet sich nun zwischen dem Halogen- und dem Wasserstoffatom. Die Konfiguration dieser drei Atome führt zu einem Übergangszustand, der die repulsive Potentialfläche der Photodissoziation von HX 
verändert. Die Potentialfläche kreuzt über eine Potentialbarriere den gebundenen Zustand des ionischen Moleküls $\mathrm{H}^{(-\alpha)} \mathrm{Rg}^{(+\beta)} \mathbf{X}^{(-\gamma)}$. Die Potentialbarriere resultiert aus dem Ladungstransfer, der der Bildung dieses stabilen Moleküls vorausgeht. Dieser Bildungsprozeß mit den beteiligten Potentialen ist in Abb. 1.10 illustriert.

Die HRgX Verbindung befindet sich nach der Bildung zwar im elektronischen Grundzustand, kann aber vibronisch angeregt sein. Die starke Wechselwirkung des ionischen Moleküls mit der Edelgasclusterumgebung führt zu einer schnellen Abregung (einige Pikosekunden) der Schwingung, die zu einer Erwärmung des Clusterkäfigs gefolgt von Abdampfung von Edelgasatomen führt [Coh01].

Bisher sind in Matrix-Experimenten folgende Moleküle entdeckt worden: $\mathrm{HXel}, \mathrm{HXeBr}$, $\mathrm{HXeCl}, \mathrm{HKrCl}, \mathrm{HKrF}, \mathrm{HArF}$ [Pet95, Pet02b, Khr00]. Für Neon gibt es auch nach theoretischen Berechnungen keine stabilen Verbindungen mit Wasserstoff und Halogenen. HXeF ist theoretisch vorausgesagt worden, seine Existenz ist experimentell aber noch nicht verifiziert. Weil es sich um eine ionische Bindung handelt, hängt die Stabilität der Verbindung vom lonisierungspotential des Edelgasatoms, der Elektronenaffinität des Halogens und der Dissoziationsenergie des Komplexes $\mathrm{H}-\mathrm{Rg}^{+}\left(D_{e}\left(\mathrm{HXe}{ }^{+}=4.05 \mathrm{eV}\right)\right.$ ab. In unserer Gruppe konnte bisher die Existenz von $\mathrm{HXel}, \mathrm{HXeBr}$ und $\mathrm{HXeCl}$ verifiziert werden, was im weiteren Verlauf dieser Arbeit diskutiert wird (Abschnitte 4.4 und 5.3).

Für die Energien der Schwingungsniveaus dieser Moleküle ist experimentell und theoretisch folgendes gefunden worden: $\nu_{\mathrm{H}-\mathrm{Rg}}=1000-1700 \mathrm{~cm}^{-1}, 2 \nu_{\mathrm{H}-\mathrm{Rg}}=1900-3000 \mathrm{~cm}^{-1}$ und $3 \nu_{\mathrm{H}-\mathrm{Rg}}=2800-5000 \mathrm{~cm}^{-1}$. Dabei führt das dritte Schwingungsniveau bei den meisten Molekülen zur Dissoziation in $\mathrm{H}+\mathrm{Rg}+\mathrm{X}$. Die Dissoziationsenergien liegen im Bereich von $0.4-1.4 \mathrm{eV}$. Weitere Daten, wie Bindungsabstände, Partialladungen, Polariserbarkeiten, Dissoziationsenergien, Rotationskonstanten und Dipolmomente für $\mathrm{HXel}, \mathrm{HXeBr}$ und $\mathrm{HXeCl}$ können Tab. 1.4 entnommen werden.

\subsubsection{Orientierung von Molekülen in kombinierten elektrischen Wechsel- und statischen Feldern}

Die elektronischen Eigenschaften der Moleküle erlauben es, sie unter bestimmten Voraussetzungen in elektrischen Wechselfeldern auszurichten oder in statischen elektrischen Feldern zu orientieren. Die elektrischen Wechselfelder lassen sich mit einem leistungsstarken, gepulsten, nichtresonanten, linear polarisierten Laser realisieren. Allgemein spricht man bei einer anisotropen axialen Verteilung von einer Orientierung (in Abb. 1.11: Pfeil 
in eine Richtung) und bei einer isotropen Verteilung von Ausrichtung (in Abb. 1.11: Pfeil in beide Richtungen). Wenn man von einem heteroatomaren Molekül wie $\mathrm{ICl}$ ausgeht und es einem elektrischen Wechselfeld (polarisierter Laser) parallel zur Detektorachse aussetzt, gibt es die beiden in Abb. 1.11 möglichen Orientierungen. Diese beiden Orientierungen sind gleich verteilt. Man spricht von einer Ausrichtung (alignment) der Molekülachse im Laserfeld [Lar99, Fri95a, Fri95b, Sei95].
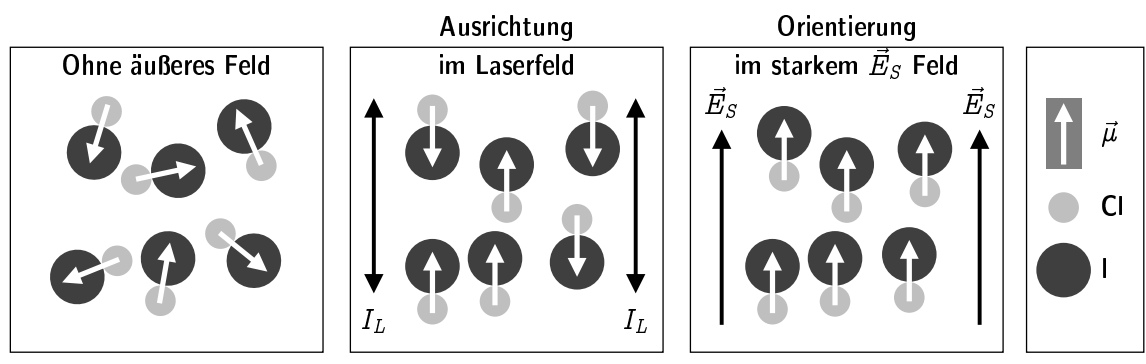

Abbildung 1.11: Ohne äußeres Feld nehmen Moleküle, hier ICl beliebige Richtungen ein (links). In einem nichtresonanten, gepulsten, linear polarisierten Laserfeld werden sie parallel zur Kernverbindungsachse ausgerichtet (alignment), (2. von links). Ein starkes, statisches elektrisches Feld ist in der Lage, Moleküle an diesem zu orientieren (3. von links). Das Dipolmoment $\vec{\mu}$ zeigt von der negativen Partialladung $\left(\mathrm{Cl}^{-}\right)$zur positiven $\left(\mathrm{I}^{+}\right)$.

Entscheidend ist hierbei die Wechselwirkung zwischen dem elektrischen Feld und dem induzierten Dipolmoment des Moleküls. Die charakteristische Größe ist der Polarisierbarkeitstensor $\alpha$.

In starken statischen Feldern lassen sich polare Moleküle an ihrem permanenten Dipolmoment $\vec{\mu}$ parallel zu $\vec{E}$ orientieren. Ein Beispiel zeigt Abb. 1.11. Voraussetzung für einen hohen Grad der Orientierung (orientation) sind niedrige Rotationszustände des Moleküls [Blo92, Kra65, Fri91, Ros92].

Dabei spielen das elektrische Dipolmoment $\vec{\mu}$ und die Rotationskonstante $B$ eine wesentliche Rolle.

Es stellt sich die Frage, wie sich eine Überlagerung von einem nichtresonanten, gepulsten, linear polarisierten Laserfeld und einem statischen elektrischen Feld auswirken. Dies wird von B. Friedrich und D.R. Herschbach beantwortet [Fri99a, Fri99b, Cai01]: Ein starkes Laserfeld, überlagert mit einem schwachen, statischen elektrischen Feld kann bei einem Molekül mit großem permanenten Dipolmoment und hohen Werten für die Polarisierbarkeiten zu einer vollständigen Orientierung am statischen Feld führen [Nah02]. Die 
Mechanismen, die bei diesen Prozessen beteiligt sind, sollen im folgenden dargelegt werden.

Der Effekt, der einer Orientierung eines Moleküls entgegenwirkt, ist seine Rotation, als Hamilton-Operator

$$
H_{\text {rot }}=B \vec{J}^{2},
$$

mit der Rotationskonstanten $B=h /\left(8 \pi^{2} c \Theta\right)$ in $\mathrm{cm}^{-1}(\Theta$ : Trägheitsmoment des Moleküls) und dem Bahndrehimpuls-Operator $\vec{J}$. Die Wechselwirkung äußerer elektrischer Felder mit dem Molekül erstreckt sich zum einen auf das permanente Dipolmoment $\vec{\mu}$, das klassisch als Produkt aus Ladungsabstand und Ladung ausgedrückt wird. Es ist eine vektorielle Größe, die von der negativen zur positiven Ladung zeigt. Zum anderen findet eine Wechselwirkung mit dem induzierten Dipolmoment statt. Das induzierte Dipolmoment $\vec{\mu}_{\text {ind }}$ ergibt sich aus dem Produkt von Polarisierbarkeit $\alpha$ und dem äußeren elektrischen Feld $\vec{E}$. Die Polarisierbarkeit $\alpha$ ist ein Maß für die Verschiebbarkeit von positiver relativ zu negativer Ladung im Molekül. Wenn man von einer beliebigen Form des Moleküls ausgeht, wird die Polarisierbarkeit $\alpha$ als Tensor geschrieben. Für lineare Moleküle läßt sich die Polarisierbarkeit auf einen Term in Richtung der Molekülachse $\alpha_{\|}$und einen senkrecht dazu $\alpha_{\perp}$ reduzieren. Homoatomare Moleküle wie $\mathrm{I}_{2}$ besitzen kein permanentes Dipolmoment, weil es ohne äußeres Feld keine Ladungsverschiebungen gibt. Bei heteroatomaren Molekülen wie $\mathrm{HI}$ existiert ein permanentes elektrisches Dipolmoment ${ }^{8}$. Bei beiden ist ein Dipolmoment induzierbar. Bei den heteroatomaren Molekülen ist selbst in statischen elektrischen Feldern von $10^{5} \mathrm{~V} / \mathrm{cm}$ das induzierte Dipolmoment um drei Größenordnungen kleiner als ihr permanentes.

Die Wechselwirkung des permanenten Dipolmoments $\vec{\mu}$ mit dem elektrischen Feld ergibt sich zu:

$$
V_{\mu}=-|\vec{\mu}||\vec{E}| \cos \theta
$$

mit dem Winkel $\theta$ zwischen elektrischem Feld und Molekülachse. Das Potential des induzierten Dipolmoments beträgt

$$
V_{\alpha}=-\frac{1}{2}|\vec{E}|^{2}\left[\left(\alpha_{\|}-\alpha_{\perp}\right) \cos ^{2} \theta+\alpha_{\perp}\right] .
$$

\footnotetext{
${ }^{8} \mu_{\mathrm{HI}}=1.9 \mathrm{D}$. Die Einheit D (Debye) gibt das elektrische Dipolmoment, in SI Einheiten As $\cdot \mathrm{m}$, an. Dabei gilt: $1 \mathrm{D}=3.336 \cdot 10^{-30}$ As $\cdot \mathrm{m}$.
} 
Angenommen sei ein Laserfeld der Stärke $E_{L}$ und ein statisches Feld $E_{S}$. Es wird ein polares Molekül mit einem permanenten Dipolmoment betrachtet. Die Wechselwirkung mit dem schnell oszillierenden Laserfeld mittelt sich zu Null $\left(V_{\mu}=-\mu E_{L}=0\right)$. Im Verhältnis zur Feldstärke des Laserfeldes ist das statische Feld nicht in der Lage, ein nennenswertes Dipolmoment zu erzeugen, so daß der Term $V_{\alpha}$ für das statische Feld vernachlässigt werden kann.

Mit Energien in Einheiten der Rotationskonstanten $B$ und den dimensionslosen Größen

$$
\omega=\frac{\mu E_{S}}{B} ; \quad \omega_{\|, \perp}=\frac{\alpha_{\|, \perp} I_{L}}{2 B} ; \quad \Delta \omega=\omega_{\|}-\omega_{\perp},
$$

wobei $I_{L}(t)=\left\langle E_{L}^{2}(t)\right\rangle$ die zeitlich gemittelte Laserintensität ist, werden die Gln. 1.42 und $1.43 \mathrm{zu}$ :

$$
\begin{aligned}
V_{\mu}\left(\omega ; \theta_{S}\right) & =-\omega \cos \theta_{S}, \\
V_{\alpha}\left(\omega_{\|} ; \omega_{\perp} ; \theta_{L}\right) & =-\left(\Delta \omega \cos ^{2} \theta_{L}+\omega_{\perp}\right) .
\end{aligned}
$$

Mit den Gln. 1.41 und 1.45 wird der Hamiltonoperator in B zu:

$$
H=\vec{J}^{2}+V_{\mu}\left(\omega ; \theta_{S}\right)+V_{\alpha}\left(\omega_{\|} ; \omega_{\perp} ; \theta_{L}\right) \quad .
$$

Die Größe $\omega$ charakterisiert die Wechselwirkung zwischen dem statischen Feld und dem permanenten Dipolmoment. $\Delta \omega$ tut dies analog für das Laserfeld. Beide lassen sich in geeigneten Einheiten ausdrücken als:

$$
\begin{aligned}
\omega & =0.0168 \mu[\mathrm{D}] E_{S}[\mathrm{kV} / \mathrm{cm}] / B\left[\mathrm{~cm}^{-1}\right], \\
\Delta \omega & =10^{-11} \Delta \alpha\left[\AA^{3}\right] I_{L}\left[\mathrm{~W} / \mathrm{cm}^{2}\right] / B\left[\mathrm{~cm}^{-1}\right] .
\end{aligned}
$$

Die quantenmechanischen Zustände, die das Molekül bei der Ausrichtung im Laserfeld einnehmen kann, bezeichnet man als Pendelzustände (pendular states). Sie werden mit den Quantenzahlen $\tilde{J}$ charakterisiert, die sich direkt aus Mischungen der Quantenzustände des feldfreien Rotors ergeben. $M$ bleibt eine gute Quantenzahl. Soweit quantenmechanische Pendelzustände einer geometrischen Anschauung zugänglich sind, kann man sie sich als eine Kombination aus behinderter Rotation um die Achse der Laserpolarisation und einer Präzession um diese Achse vorstellen. Die Amplitude der behinderten Rotation nimmt 
mit stärkerem Laserfeld ab. Eine andere Bezeichnung für diesen Effekt ist die Libration (libration).

Der Übergang des quantenmechanischen Systems als freier Rotor $|J M\rangle$ in die Pendelzustände $|\tilde{J} M\rangle$, generiert durch das Laserfeld, ist nur adiabatisch möglich. Dies setzt Anstiegszeiten und Pulsdauern des Lasers voraus, die vergleichsweise lang zu einer Rotationsperiode des Moleküls sind. Für typische Rotationskonstanten von $B \approx 0.1 \mathrm{~cm}^{-1}$ läßt sich dieser adiabatische Übergang nur mit ns-Laserpulsen erreichen. Mit einem fs-Laser ist dieses Phänomen nicht beobachtbar.

Der Ausrichtungsgrad wird durch den Erwartungswert von $\cos ^{2} \theta_{L}$ aus GI. 1.45, $\left\langle\cos ^{2} \theta_{L}\right\rangle$ angegeben. Wenn der Quantenzustand eine isotrope Verteilung hervorbringt, resultiert dies in $\left\langle\cos ^{2} \theta_{L}\right\rangle=1 / 3$, währenddessen ein vollständig ausgerichteter Zustand zu $\left\langle\cos ^{2} \theta_{L}\right\rangle=1$ führt. Aus der Lösung der Schrödinger-Gleichung 1.46 erhält man $\left\langle\cos ^{2} \theta_{L}\right\rangle$. Die Energieeigenwerte zeigen die Konkurrenz zwischen den freien Rotationszuständen und den Pendelzuständen. Eine sehr gute Ausrichtung des Moleküls ist nur für kleine $\tilde{J}$ möglich, was man mit der Wahl einer niedrigen Rotationstemperatur erreichen kann. In einer Düsenstrahlexpansion setzt dies eine geeignete Präparation des Molekularstrahls voraus (Koexpansionsgas, Expansionsdruck, Düsendurchmesser) [Lar99].

Schon die Überlagerung eines schwachen statischen Feldes parallel zum Laserfeld und dem darin ausgerichteten Molekül kann zu einer Orientierung desselben führen. Wenn wir davon ausgehen, daß die Amplitude der Pendelschwingung als Konsequenz von Kreuzungen der $\tilde{J}$ Zustände stark reduziert ist, resultieren dadurch energetisch schwach getrennte, fast entartete $\tilde{J}$ Zustands-Paare, sogenannte Tunnel-Dubletts. In diesen Zuständen führt selbst die Überlagerung eines schwachen statischen elektrischen Feldes zur Aufhebung der Entartung und damit zu einem hohen Orientierungsgrad des Moleküls. Diesen Mechanismus, eine Art Linienaufspaltung, bezeichnet man als Pseudo-linearen Stark Effekt (pseudo-first-order Stark effect).

Zu jedem Zustand $\left|\tilde{J}_{i} M_{j}\right\rangle$ gibt es einen energetisch nur leicht verschiedenen Zustand $\left|\tilde{J}_{k} M_{l}\right\rangle$, der zu einer Orientierung in entgegengesetzte Richtung führt. Der Grad der Orientierung wird mit dem Erwartungswert von $\cos \theta_{S}$ aus GI. 1.45 mit $\left\langle\cos \theta_{S}\right\rangle$ angegeben. Er kann Werte zwischen -1 und 1 annehmen, wobei $\cos \theta=1$ einer vollständigen Orientierung in Richtung des elektrostatischen Feldes entspricht.

Die in diesem Abschnitt behandelten Moleküle der Klasse HRgX $(\mathrm{Rg}=\mathrm{Ar}, \mathrm{Kr}$, Xe; $\mathrm{X}=\mathrm{Cl}, \mathrm{Br}, \mathrm{I})$ sind lineare, polare Moleküle. Das besondere Interesse gilt dabei den Spezies $\mathrm{HXeX}$, weil Xe eine sehr hohe Polarisierbarkeit $\left(\alpha=4.04 \AA^{3}\right)$ besitzt. In der Kombination 
mit lod, das ebenfalls eine hohe Polarisierbarkeit besitzt $\left(\alpha=6.2 \AA^{3}\right)$, erzeugt dies eine starke Anisotropie in der Polarisierbarkeit des Moleküls von $\Delta \alpha=5.5 \AA^{3}$, woraus ein hohes induzierbares Dipolmoment resultiert. ${ }^{9}$ Die Ladungstrennung zwischen $\mathrm{X}$ und $\mathrm{Rg}$, verbunden mit den großen Bindungsabständen $r_{X-R g}$, bewirkt ebenfalls hohe permanente Dipolmomente $\left(\mu_{\mathrm{HXel}}=6.41 \mathrm{D}\right)$, die eine Orientierung in statischen elektrischen Feldern vereinfachen. Weitere Daten zu dieser Art von Molekülen sind in Tab. 1.4 zu finden.

${ }^{9}$ Bei der Bestimmung von $\Delta \alpha$ wurde die Relation zwischen $\Delta \alpha$ und $\alpha$ bei den ähnlichen Molekülen $\mathrm{I}_{2}$ und $\mathrm{Br}_{2}$ benutzt. G. Maroulis gibt einen Überblick über die Definition von Polarisierbarkeiten und hat die Werte für HI berechnet [Mar00]. 


\section{Experimenteller Aufbau}

Die in dieser Arbeit verwendete Apparatur wurde ursprünglich als Kreuzstrahlapparatur zur Analyse von Clustergrößenverteilungen und zur Präparation größenselektierter Clusterstrahlen gebaut [Loh01, Bau95, Loh93]. Die Apparatur wurde durch ein Wiley-McLaren Flugzeitmassenspektrometer und durch zwei Lasersysteme ergänzt. Dies ermöglicht Photodissoziationsexperimente an Clustern durchzuführen und die Fragmente mittels Laserionisation im Flugzeitmassenspektrometer nachzuweisen [Bau99a, Nah98, Fri97].

Die drei wesentlichen Einheiten dieser Apparatur sollen in diesem Kapitel kurz diskutiert werden. Diese sind im einzelnen die Molekularstrahlapparatur zur Erzeugung und Präparation der Clusterstrahlen, die Lasersyteme zur Dissoziation der Moleküle in den Clusterumgebungen und zur lonisation der Fragmente sowie verschiedene Ausführungen von Wiley-McLaren Flugzeitmassenspektrometern zum Nachweis der Fragmente.

Auf die wesentlichen Neuerungen gegenüber früheren Experimenten wird in den einzelnen Abschnitten näher eingegangen:

- Tieftemperatur-Clusterquelle zur Erzeugung großer Ne Cluster.

- Gepulstes Wiley-McLaren Flugzeitmassenspektrometer.

- Wiley-McLaren Flugzeitmassenspektrometer mit längerer, abgeschirmter Driftstrecke. Umstellung des Detektors von MCP auf MSP ${ }^{1}$.

- Einsatz eines neuen Dissoziationslasers mit $193 \mathrm{~nm}$ Strahlung. Polarisierung (inkl. Drehung der Polarisationsebene) und Führung in einem gekapselten, spül- oder evakuierbaren Strahlengang.

${ }^{1} \mathrm{MCP}:$ multi channel plates oder Vielkanalplatten. MSP: micro sphere plates oder MikroOberflächenplatten. Bei beiden handelt es sich um sehr effektive Sekundärelektronenvervielfacher (SEV). 


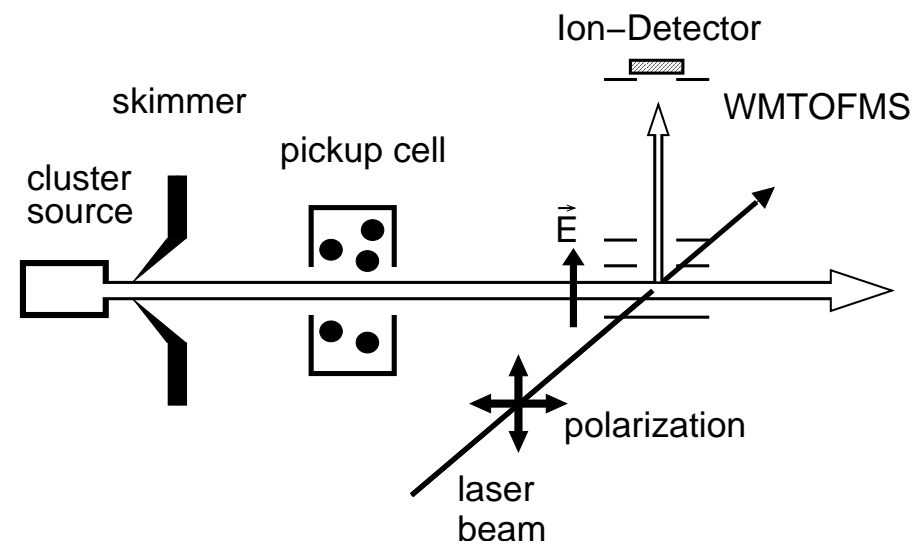

Abbildung 2.1: Schematischer Aufbau des Experiments: cluster source: mittels Düsenstrahlexpansion erzeugter Clusterstrahl mit puren Edelgasclustern oder bei Koexpansion mit $\operatorname{Rg}_{n}(\mathrm{HX})_{\times}$Mischclustern; pickup cell: Anlagerung von Molekülen an die Cluster im Strahl; WMTOFMS: Wiley-McLaren Flugzeitmassenspektrometer im Niedrigfeldmodus; laser beam: Laserstrahl mit $243 \mathrm{~nm}$ zur Dissoziation der Moleküle und lonisation der Wasserstofffragmente oder zusammen mit der Dissoziationswellenlänge von $193 \mathrm{~nm}$ nur zur lonisation; polarisation: Die Laserstrahlung von $243 \mathrm{~nm}$ ist je nach Experiment senkrecht oder parallel zur Detektorachse polarisiert, ebenso für $193 \mathrm{~nm}$, hier aber auch häufig unpolarisiert; $\vec{E}$ : Richtung des lonenabzugsfeldes im WMTOFMS; Ion-Detector: MCP oder MSP zum Nachweis der Wasserstoffionen.

Eine schematische Skizze der experimentellen Anordnung ist in Abb. 2.1 dargestellt. Der Clusterstrahl wird in einer Düsenstrahlexpansion des reinen Edelgases oder einer EdelgasHalogen-Mischung mit einer konischen Düse erzeugt. Die Clustergröße wird dabei über Düsentemperatur und Expansionsdruck variiert (s. Abschnitt 1.2.2). Der Clusterstrahl verläßt die Quellenkammer durch einen skimmer (Strahlabschäler) und eine Pick-up-Zelle, in der die Cluster mittels Moleküleinfang mit Chromophoren dotiert werden können. AnschlieBend gelangen die präparierten Cluster in das Wiley-McLaren-Flugzeitmassenspektrometer (WMTOFMS). Zusätzlich werden hier die Laserstrahlen zur Dissoziation und Ionisation fokussiert eingekoppelt. Der Molekularstrahl, die Strahlachse des Dissoziationslasers und die Detektorachse kreuzen sich im Zentrum des WMTOFMS und spannen dort ein rechtshändiges Koordinatensystem auf. Damit werden Doppler-Effekte in den Photodissoziationsmessungen vermieden.

Die Moleküle in den Clusterumgebungen werden im WMTOFMS photodissoziiert. Die Fragmente werden ionisiert und energiesensitiv im WMTOFMS, das im Niedrigfeldmodus arbeitet, nachgewiesen. 


\title{
2.1 Molekularstrahlapparatur
}

Ein horizontaler und vertikaler Schnitt durch die Molekularstrahlapparatur sind in Abb. 2.2 dargestellt.

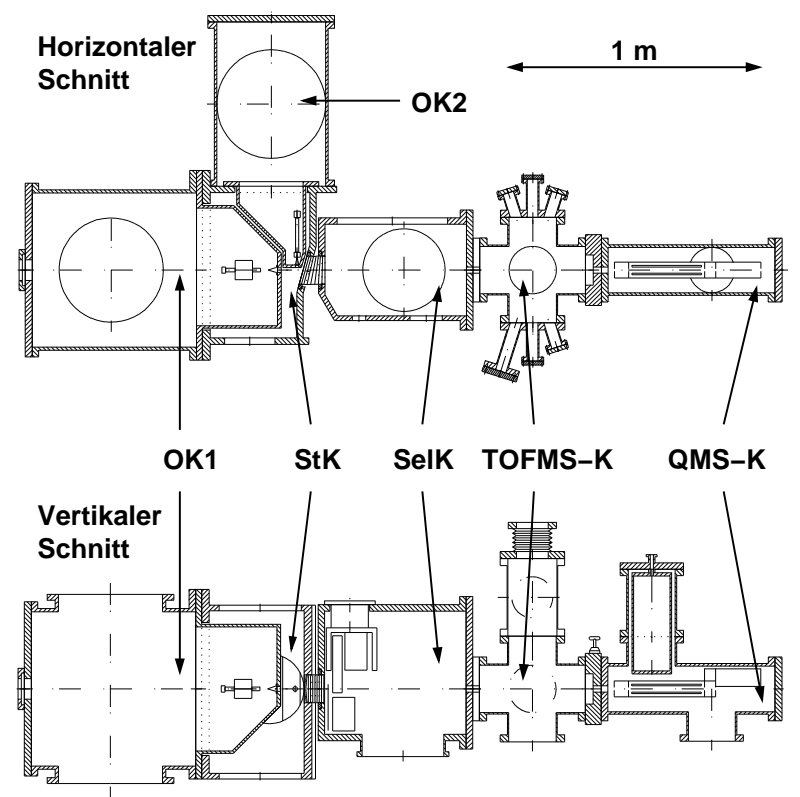

\begin{abstract}
Abbildung 2.2: Molekularstrahlapparatur im horizontalen und vertikalen Schnitt, OK1 Ofenkammer 1, OK2 Ofenkammer 2, StK Streukammer, SelK Selektorkammer, TOFMS-K Kammer mit Flugzeitmassenspektrometer, QMS-K Kammer mit Quadrupolmassenspektrometer
\end{abstract}

Für die hier beschriebenen Experimente findet die Ofenkammer 2 (OK2) keine Verwendung. Gleiches gilt im Prinzip auch für die Streukammer (StK), die allerdings zur Verbesserung der Strahleigenschaften als differentielle Pumpstufen arbeitet ${ }^{2}$. In der Selektorkammer (SelK) befindet sich die Pick-up-Zelle sowie ein Pseudo-Random-Chopper, der sich bei Bedarf in den Strahl fahren läßt. Er wird in Kombination mit dem Nachweis im Quadrupolmassenspektrometer zur Messung der Geschwindigkeitsverteilung des Mo-

\footnotetext{
${ }^{2}$ In früheren Experimenten wurde die Maschine zur Clusterstrahlanalyse und Größenselektion genutzt. Aus diesem Grund ist sie als Kreuzstrahlapparatur (Strahlquelle 1 in OK1 und Strahlquelle 2 in OK2) konstruiert, bei der OK1, OK2 und StK auf einem Drehkranz gelagert sind. Drehmittelpunkt ist das Streuzentrum. In der Selektorkammer kann ein Einscheiben-Geschwindigkeitsselektor zur vollständigen Größenselektion in den Strahl gefahren werden [Bau95, Fri97].
} 
lekularstrahls benötigt. Die beiden Massenspektrometer befinden sich jeweils in eigenen Kammern und werden hier auch in separaten Abschnitten beschrieben (Wiley-McLaren Flugzeitmassenspektrometer in der TOFMS-K (Abschnitt 2.3) und das Quadrupolmassenspektrometer in der QMS-K (Abschnitt 2.1.3).

\begin{tabular}{|c|c|c|c|c|c|}
\hline Kammer & $\begin{array}{l}\text { Vorpumpe } \\
\text { 1. Stufe }\end{array}$ & $\begin{array}{l}\text { Vorpumpe } \\
\text { 2. Stufe }\end{array}$ & $\begin{array}{c}\text { HV- bzw. } \\
\text { UHV-Pumpe }\end{array}$ & $\begin{array}{l}\text { zusätzliche } \\
\text { Pumpstufen }\end{array}$ & $\begin{array}{c}\text { Druck während } \\
\text { Betrieb } p_{w}\end{array}$ \\
\hline Ofenkammer 1 & $\begin{array}{c}\text { Drehschieber } \\
\text { Alcatel } \\
\text { T } 2100 \mathrm{AC} \\
100 \mathrm{~m}^{3} / \mathrm{h}\end{array}$ & $\begin{array}{c}\text { Wälzkolben } \\
\text { Pfeiffer } \\
\text { WKP } 1000 \\
1000 \mathrm{~m}^{3} / \mathrm{h}\end{array}$ & $\begin{array}{c}\text { Diffusion } \\
\text { Leybold } \\
\text { DI } 6000 \\
60001 / \mathrm{sec}\end{array}$ & $\begin{array}{c}\mathrm{N}_{2} \text {-Kühlfalle } \\
\text { / Kaltkopf } \\
\text { Leybold } \\
\text { RGD } 1245\end{array}$ & $4 \cdot 10^{-4} \mathrm{mbar}$ \\
\hline Ofenkammer 2 & $\begin{array}{c}\text { Drehschieber } \\
\text { Edwards } \\
\text { E } 1 \mathrm{M} 175 \\
175 \mathrm{~m}^{3} / \mathrm{h}\end{array}$ & $\begin{array}{c}\text { Wälzkolben } \\
\text { Leybold } \\
\text { WS } 500 \\
500 \mathrm{~m}^{3} / \mathrm{h}\end{array}$ & $\begin{array}{l}\text { Diffusion } \\
\text { Leybold } \\
\text { DI } 6000 \mathrm{E} \\
60001 / \mathrm{sec}\end{array}$ & & $4 \cdot 10^{-4} \mathrm{mbar}$ \\
\hline Streukammer & $\begin{array}{c}\text { Drehschieber } \\
\text { Alcatel } \\
\text { T2030 } \\
30 \mathrm{~m}^{3} / \mathrm{h}\end{array}$ & & $\begin{array}{l}\text { Diffusion } \\
\text { Varian } \\
\text { VHS } 6 \\
27001 / \mathrm{sec}\end{array}$ & $\mathrm{N}_{2}$-Kühlfalle & $5 \cdot 10^{-6} \mathrm{mbar}$ \\
\hline Selektorkammer & $\begin{array}{c}\text { Drehschieber } \\
\text { Alcatel } \\
\text { T2030 } \\
30 \mathrm{~m}^{3} / \mathrm{h} \\
\end{array}$ & & $\begin{array}{l}\text { Diffusion } \\
\text { Varian } \\
\text { VHS } 6 \\
27001 / \mathrm{sec} \\
\end{array}$ & $\mathrm{N}_{2}$-Kühlfalle & $5 \cdot 10^{-7} \mathrm{mbar}$ \\
\hline $\begin{array}{l}\text { TOFMS- } \\
\text { Kammer }\end{array}$ & $\begin{array}{c}\text { Drehschieber } \\
\text { Edwards } \\
\text { E } 2 \mathrm{M} 18 \\
18 \mathrm{~m}^{3} / \mathrm{h}\end{array}$ & & $\begin{array}{l}\text { Turbomolekular } \\
\text { Balzers } \\
\text { TPU 520M } \\
5001 / \mathrm{sec}\end{array}$ & $\begin{array}{l}\text { Kaltkopf } \\
\text { Leybold } \\
\text { RGD } 580\end{array}$ & $4 \cdot 10^{-8} \mathrm{mbar}$ \\
\hline QMS-Kammer & $\begin{array}{c}\text { Drehschieber } \\
\text { Edwards } \\
\text { E2M8 } \\
8 \mathrm{~m}^{3} / \mathrm{h}\end{array}$ & $\begin{array}{l}\text { Diffusion } \\
\text { Balzers } \\
\text { DIF 063I } \\
150 \mathrm{l} / \mathrm{sec}\end{array}$ & $\begin{array}{c}\text { Turbomolekular } \\
\text { Balzers } \\
\text { TPU } 520 \\
5001 / \mathrm{sec}\end{array}$ & $\mathrm{N}_{2}$-Kühlfalle & $2 \cdot 10^{-9} \mathrm{mbar}$ \\
\hline
\end{tabular}

Tabelle 2.1: Vakuumerzeugung in den einzelnen Kammern der Molekularstrahlapparatur durch differentielle Pumpstufen. Die Angaben zu den Pumpen sind jeweils Pumpentyp, Hersteller, Typenbezeichnung und Nennsaugvermögen. Weiter ist der Druck in den jeweiligen Kammern während der Strahlerzeugung angegeben.

Jede der oben aufgeführten Kammern wird durch ein mehrstufiges Pumpensystem evakuiert. Als Vorvakuumpumpen kommen Drehschieber- und Wälzkolbenpumpen zum Einsatz. Das Hochvakuum (HV) bzw. Ultrahochvakuum (UHV) wird mit Öldiffusionsund Turbomolekularpumpen erzeugt. Die verwendeten Pumpentypen und damit erreichten Drücke in den unterschiedlichen Kammern sind in Tabelle 2.1 aufgeführt. 
Die Wahl der Pumpentypen hängt von der Anwendung in der jeweiligen Kammer ab. Der durch die Düsenstrahlexpansion hohe Gasanfall in den Ofenkammern kann nur durch zusätzlichen Einsatz von Wälzkolbenpumpen bewältigt werden. Die Drehschieberpumpe der Ofenkammer 1 wird mit einem Ölfiltersystem für korrosive Gase (hier Molekularstrahl $5 \% \mathrm{HBr}$ in $\mathrm{Ar}$ ) vor Defekten geschützt. In der Flugzeitmassenspektrometer-Kammer ist ein möglichst niedriger Kohlenwasserstoffuntergrund erwünscht. Dies ist mit Öldiffusionspumpen, aufgrund ihrer mineralölhaltigen Treibmittel, nicht erreichbar. Deswegen kommt hier eine Turbomolekularpumpe (TMP) zum Einsatz. Eine weitere Reduktion des Untergrundes wird durch Einsatz eines He-Kaltkopfes (Leybold RGD580) erreicht, der die meisten Gase ausfriert. An der Quadrupolmassenspektrometer-Kammer befindet sich vor der Turbomolekularpumpe eine kleine Diffusionspumpe, die den Durchsatz leichter Gase wie $\mathrm{He}$ verbessert.

Auch in den anderen Kammern sind Kryopumpen in Form von Kühlfallen integriert, um Restgase auszufrieren. In der Ofenkammer 1 ist dies je nach verwendeter Clusterquelle eine Stickstoffkühlfalle oder ebenfalls ein He-Kaltkopf (Leybold RGD1245). In den übrigen Kammern befinden sich Stickstoffkühlfallen.

\subsubsection{Quelle zur Erzeugung großer Ne Cluster}

Die Molekularstrahl- und Clustererzeugung ist in ihren theoretischen Grundlagen in $\mathrm{Ab}$ schnitt 1.2 dargelegt. Die Düsenstrahlexpansion der Edelgase oder Edelgas-Halogen Gemische in diesen Experimenten geschieht durch konische Düsen, wobei die Konuslänge typischerweise $2 \mathrm{~mm}$ beträgt. Der Öffnungswinkel variiert zwischen $20^{\circ}$ und $40^{\circ}$. Der kleinste Durchmesser des Konus bewegt sich im Bereich 30-60 $\mu \mathrm{m}$. Die verwendeten Düsen sind jeweils direkt in der Parameterliste der experimentellen Ergebnisse (Kapitel 4) aufgeführt.

Wie in Abschnitt 1.2.2 beschrieben, kann die Clustergröße über die Änderung von Expansionsdruck und Quellentemperatur variiert werden. In den meisten dieser Experimente kommt eine Quelle zum Einsatz, bei der die Düse über eine Kupferverbindung zur Stickstoffkühlfalle in der Ofenkammer 1 gekühlt wird. Die Kontaktfläche und die Beschaffenheit der Kupferlitze sind soweit optimiert worden, daß eine minimale Düsentemperatur von $158 \mathrm{~K}$ erreicht werden kann. Diese Kühlung läßt nur eine feste Arbeitstemperatur zu, so daß für die Temperaturvariation eine elektrische Heizung mit einer Regelung eingesetzt wird. Diese Regelung besteht aus einem Prozeßregler (Hartmann \& Braun, Protronic 500) und einem regelbaren Netzteil (Knürr-Heinzinger, LNG 50-4). Die maximal erreichbare Düsentemperatur beträgt mit diesem Aufbau $400 \mathrm{~K}$ 


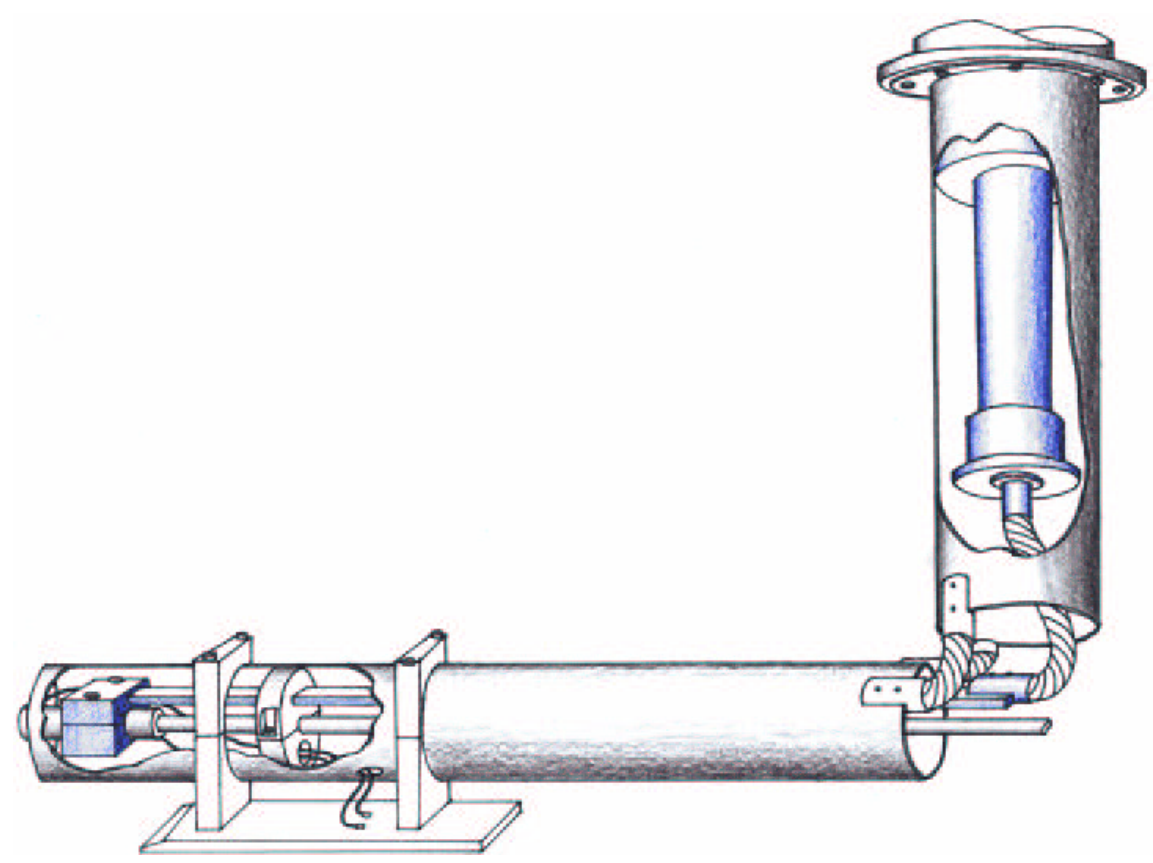

Abbildung 2.3: Quelle zur Erzeugung großer Ne Cluster. In der horizontalen Ebene befindet sich ganz links die Düse mit einem dünnen Edelstahlrohr als Zuleitung. Der Düsenkörper ist vorne mit einem Kupferblock (blau) umgeben. Dieser ist mit einer Kupferstange (blau) und einer -litze mit der zweiten Stufe des He-Kaltkopfs verbunden (blau, senkrecht). Die gesamte Anordnung ist von Kupferrohren umgeben, die mit der ersten Stufe des Kaltkopfes verbunden sind. Sie verhindern thermische Einstrahlung aus der Kammer auf die Quelle. Zur thermischen Isolierung sind die Halterungen für die Rohre, die Kupferstange und die Gaszuleitung aus Teflon gefertigt. Die elektrischen Zuleitungen für die Gegenheizung werden durch eine Öffnung in der Kupferabschirmung hinausgeführt. Die horizontale Ausdehnung der Quelle beträgt ungefähr $40 \mathrm{~cm}$. Zeichnung mit freundlicher Genehmigung von M. Fárník.

Cluster aus den Edelgasen $\mathrm{Ar}, \mathrm{Kr}$ und $\mathrm{Xe}$ lassen sich in dieser Quelle bis zu Größen von einigen hundert Atomen erzeugen. Dagegen ist diese Anordnung nicht zur Herstellung von Ne Clustern geeignet. In ersten Experimenten hat R. Baumfalk eine Quelle mit stickstoffumspülter Düse benutzt, mit der minimale Temperaturen von $110 \mathrm{~K}$ erreicht werden können. Die größten Ne Cluster, die sich bei sonstiger Optimierung der Parameter (Düsendurchmesser und -konus, Expansionsdruck, Pumpleistung) auf diese Weise erzeugen lassen, besitzen eine mittlere Clustergröße von $\langle n\rangle=150$.

Diese Limitierung sollte hier aufgehoben werden. Dafür wurde eine neue Quelle konstruiert, die zur Kühlung anstatt flüssigem Stickstoff einen zweistufigen He Kaltkopf (Leybold 
RGD1245 mit He Kompressor Leybold RW4000EU) benutzt. Eine schematische dreidimensionale Ansicht der Quelle ist in Abb. 2.3 zu sehen. Zur Kühlung wird der He Kaltkopf anstatt der Stickstoffkühlfalle in die Ofenkammer 1 eingebaut, wobei beide Stufen genutzt werden. Die erste Stufe mit einer Kühlleistung von $45 \mathrm{~W}$ (bei $80 \mathrm{~K}$ ) kühlt die KupferAbschirmung der eigentlichen Düsenkühlung. Die Düse ist über eine Kupferstange/-litze mit der zweiten Stufe des Kaltkopfes verbunden, die $12 \mathrm{~W}$ Kühlleistung aufbringt (bei $20 \mathrm{~K}$ ). Die minimal erreichbare Düsentemperatur beträgt bei diesem Aufbau $45 \mathrm{~K}$. Damit lassen sich Ne Cluster mit mittleren Clustergrößen $\langle n\rangle>2000$ erzeugen. Zur Regelung der Düsentemperatur kommt die oben erwähnte Gegenheizung zum Einsatz, die einen Temperaturbereich von $45 \mathrm{~K}<T_{D}<200 \mathrm{~K}$ abdeckt.

In den hier durchgeführten Experimenten kommt die Quelle sowohl zur Erzeugung großer Ne Cluster $(\langle n\rangle>200)$ als auch zur Erzeugung großer Ar Cluster $(\langle n\rangle>400)$ zum Einsatz.

\subsubsection{Clusterpräparation}

Von besonderem Interesse in dieser Arbeit ist die Präparation der Edelgascluster. Sie dienen als Umgebung, in die die zu untersuchenden Moleküle eingebettet werden, zum einen durch Koexpansion, zum anderen durch Pick-up (beides Abschnitt 1.2.3).

Die Gasmischungen für die Koexpansion werden üblicherweise durch Mehrfachverdünnung in Edelstahlgaszylindern (Hoke 8HD3000, max. 124 bar) hergestellt. Um Fehler in der Gaskonzentration zu vermeiden, wird zuerst von einer $5 \%$ Mischung ausgegangen, die dann nach Bedarf in ein oder zwei Schritten auf Konzentrationen bis $0.05 \%$ Halogenwasserstoff in einem Edelgas verdünnt wird.

Um die Halogenwasserstoffmoleküle im Anschluß an die Expansion in die Clusterumgebung einbetten zu können, passiert der Edelgasclusterstrahl eine Pick-up-Zelle, die sich am Eingang der Selektorkammer befindet. Diese Zelle besteht aus Edelstahlrohren mit $20 \mathrm{~mm}$ Durchmesser, die aufgrund der Plazierung in dem Faltenbalg zwischen Streukammer und Selektorkammer eine T-förmige Konstruktion erhielt: Der horizontale Arm der Zelle ragt in den Faltenbalg, und die vertikalen Arme dienen der Befestigung an der Selektorkammerwand sowie zur Aufnahme der Gaszuleitung. Eine der Molekularstrahldivergenz angepaßte Geometrie der Zelle wird erreicht, indem als Öffnungsdurchmesser am Strahleingang $4 \mathrm{~mm}$ und am Ausgang $5 \mathrm{~mm}$ gewählt wurden. Die Gesamtweglänge des Molekularstrahls innerhalb der Zelle beträgt $50 \mathrm{~mm}$ (Wechselwirkungslänge). 
Der Gaseinlaß in die Pickup-Zelle wird über ein Nadelventil Hoke 1600 Serie: Micromite) reguliert. Der dabei in diesen Experimenten benutzte Druckbereich von $2 \cdot 10^{-2} \mathrm{mbar}$ bis $8 \cdot 10^{-2}$ mbar wird mittels einer Thermovacröhre (Leybold, TR 211) bestimmt. Die Zuleitungen des verwendeten Gaseinlasses bestehen aus Edelstahlröhren und sind mit Edelstahlquetschverbindungen (Hoke Gyrolok) verbunden, da wegen der Verwendung unverdünnter Halogenwasserstoffe mit besonderer Vorsicht vorgegangen werden muß. Als weitere Vorsichtsmaßnahme wird jeweils nur ein kleines Volumen der Zuleitungen mit Halogenwasserstoffen unter hohem Druck befüllt und nach Befüllung die Gasflasche sofort wieder durch ein Ventil vom Leitungssystem getrennt. Für jedes der drei $\mathrm{Gase}(\mathrm{HCl}, \mathrm{HBr}, \mathrm{HI})$ gibt es ein getrenntes Zuleitungssystem. Dies vermeidet Beeinflussungen der Messungen durch resistente Verschmutzungen der Oberflächen.

\subsubsection{Strahlanalyse mittels Quadrupolmassenspektrometer}

Nach der Erzeugung eines Clusterstrahls versucht die Strahlanalyse, Strahleigenschaften wie die Geschwindigkeit der Teilchen im Strahl oder im Fall von Clusterstrahlen die Zusammensetzung des Strahls, seine Clustergrößenverteilung, zu bestimmen. Insbesondere bei Strahlen aus neutralen Clustern stellt sich hierbei das Problem, daß ein Nachweis durch lonisation - wie in unserem Fall in einem Quadrupolmassenspektrometer durch Elektronenstoß - die Größenverteilung durch Fragmentierung verändert. Eine Möglichkeit, sowohl diese Fragmentierung der Cluster zu bestimmen als auch einzelne Clustergrößen vollständig zu separieren, bietet die Methode der Stoßselektion [Buc84] mit anschließender Geschwindigkeitsselektion. Da diese Methode in den hier vorgestellten Messungen nicht verwendet wurde, jedoch im Experiment zur Verfügung steht, sei auf frühere Arbeiten verwiesen [Bau95, Loh93].

Sowohl bei Pseudo-Random-TOF-Messungen als auch zur Bestimmung von Fragmentierungsspektren dient ein Quadrupolmassenspektrometer als Monitordetektor zur Strahlanalyse. Die charakteristischen Eigenschaften des Spektrometersystems, wie Kalibrierungsmessungen mit Eichgasen und die Bestimmung des Transmissionsverhaltens, werden in [Loh93, Bau95] dargelegt. Es folgt hier ein kurzer Überblick über dieses Detektorsystem.

In der Raumladungsionenquelle (Extranuclear Laboratories: Modell 041-1) werden die neutralen Cluster oder Moleküle durch Elektronenstoß (mit einer Elektronenenergie von in der Regel $70 \mathrm{eV}$ ) ionisiert. Die entstandenen lonen werden von der lonenoptik abgezogen, kollimiert und in Richtung des Quadrupolstabsystems (Extranuclear Laboratories: Modell 
162-5) beschleunigt. Die zwischen den vier Stäben angelegte Spannung, ein Gleichspannungsanteil überlagert von einer RF-Wechselspannung, selektiert lonen einer bestimmten spezifischen Masse $m / e$, die das Stabsystem passieren und im Sekundärelektronenvervielfacher (Channeltron von Galileo: Modell 4840G) nachgewiesen werden. Ein Ablenkfeld hinter dem Stabsystem ermöglicht eine off-axis-Anordnung des Channeltrons, so daß zum einen neutrale Teilchen nicht unnötig auf das Channeltron prallen und zum anderen eine optische Justage der Molekularstrahlachse im evakuierten Zustand der Apparatur durchgeführt werden kann.

Das Channeltron liefert um einen nominellen Faktor von $\approx 10^{8}$ verstärkte Spannungspulse (bei 3000 V Versorgungsspannung). Die weitere Verstärkung, Konvertierung und Diskriminierung der Signale über CAMAC-Module und NIM-BIN-Einschübe wird von der Apparaturelektronik ausgeführt, die basierend auf einem CAMAC-Bussystem mit dem zentralen Apparaturrechner verbunden ist.

\subsection{Lasersysteme}

Die effektive Einphotonenanregung zur Dissoziation von Halogenwasserstoffen umfaßt den Wellenlängenbereich 140-300 nm (Absorptionsquerschnitte s. Abb. 1.4). Wellenlängen oberhalb von $200 \mathrm{~nm}$ können mit durchstimmbaren Lasersystemen durch anschließende nichtlineare Frequenzkonversionen in Kristallen erreicht werden. Für Wellenlängen unterhalb von $200 \mathrm{~nm}$ bieten sich nichtlineare Frequenzkonversionen in Edelgasen und Metalldämpfen an [Nah98]. Diese Methode zur Erzeugung durchstimmbarer Laserstrahlung im Bereich des VUV (Vakuum-Ultraviolett) ist allerdings sehr uneffektiv und erlaubt maximale Pulsenergien von einigen Mikrojoule. Allerdings stehen in diesem Bereich durch festfrequente Excimerlaser die Wellenlängen von $193 \mathrm{~nm}$ (ArF Excimer) und $157 \mathrm{~nm}\left(\mathrm{~F}_{2}\right.$ Excimer) mit maximalen Pulsenergien von $450 \mathrm{~mJ}$ zur Verfügung.

Die resonant verstärkte Mehrphotonenionisation wird in diesen Experimenten über den $2+1$ Prozeß bei $243 \mathrm{~nm}$ durchgeführt. Dieser besitzt einen geringeren Querschnitt als der $1+1$ Prozeß $(121 \mathrm{~nm})$, der in früheren Arbeiten unserer Gruppe zum Einsatz kam [Nah98, Bau99b]. Für diesen Prozeß sprechen aber seine im Vergleich einfachere technische Handhabung und die deutlich höheren Pulsenergien (3 mJ vs. $50 \mathrm{~nJ}$ ), die das Defizit im Querschnitt vollständig ausgleichen.

In dieser Arbeit kommen zwei Lasersysteme zum Einsatz. Ein durchstimmbares Nd:YAG-Farbstofflaser-System und ein Excimerlaser, der bei $193 \mathrm{~nm}$ und $157 \mathrm{~nm}$ betrieben 


\begin{tabular}{|l|c|c|c|c|}
\hline Laser & Pulsenergie & $\begin{array}{c}\text { Bandbreite } \\
{\left[\mathbf{c m}^{-1}\right]}\end{array}$ & $\begin{array}{c}\text { Pulslänge } \\
{[\mathbf{n s}]}\end{array}$ & Polarisation \\
\hline \hline $\begin{array}{l}\text { Nd:YAG-Laser } \\
\text { (GCR-5, Spectra Physics) }\end{array}$ & $\begin{array}{c}1.4 \mathrm{~J} \text { bei } 1064 \mathrm{~nm} \\
730 \mathrm{~mJ} \text { bei } 532 \mathrm{~nm}\end{array}$ & $<0.04$ & $\begin{array}{c}8 \text { bei } 1064 \mathrm{~nm} \\
6 \text { bei } 532 \mathrm{~nm}\end{array}$ & linear \\
\hline $\begin{array}{l}\text { Farbstofflaser } \\
(\text { LDL 20505, LAS) }\end{array}$ & $160 \mathrm{~mJ}$ bei $630 \mathrm{~nm}$ & 0.04 & 6 & linear \\
\hline $\begin{array}{l}\text { Excimer-Laser } \\
(\text { LPF 202, Lambda) }\end{array}$ & $\begin{array}{c}460 \mathrm{~mJ} \text { bei } 193 \mathrm{~nm} \\
80 \mathrm{~mJ} \text { bei } 157 \mathrm{~nm}\end{array}$ & 260 & 20 & unpolarisiert \\
\hline
\end{tabular}

Tabelle 2.2: Kenndaten der Lasersysteme: Nd:YAG-Laser, Farbstofflaser und Excimer-Laser

werden kann. Das Nd:YAG-Farbstofflaser-System erzeugt für alle Experimente in dieser Arbeit Licht mit einer Wellenlänge von $243 \mathrm{~nm}$. Dies wird in allen Experimenten zur lonisation der Wasserstofffragmente benutzt. Zusätzlich dient es in den Einfarbenexperimenten zur Dissoziation von $\mathrm{HI}$ und $\mathrm{HBr}$. Zur Photodissoziation von $\mathrm{HCl}$ wird in einem Zweifarbenexperiment der Excimerlaser bei einer Wellenlänge von $193 \mathrm{~nm}$ eingesetzt. Die technischen Daten der Laser sind in Tab. 2.2 aufgeführt.

Die Lasersysteme sollen hier nur kurz dargestellt werden. Eine ausführliche Beschreibung des Nd:YAG-Fabstofflaser-Systems befindet sich in [Fri97]. Die exakte räumliche und zeitliche Überlagerung zweier Laserstrahlen (Dissoziation und lonisation) ist in [Nah98] beschrieben.

\subsubsection{Lasersystem zur Erzeugung von $243 \mathrm{~nm}$}

Eine Skizze des Nd:YAG-Farbstofflaser-Systems zur Erzeugung von Laserstrahlung mit einer Wellenlänge von $243 \mathrm{~nm}$ ist in Abb. 2.4 dargestellt. Der Nd:YAG Laser (Spectra Physics: Quanta Ray GCR-5) erzeugt Laserstrahlung von $1064 \mathrm{~nm}$. Diese wird in einem KD*P-Kristall $\left(\mathrm{KD}_{2} \mathrm{PO}_{4}\right)$ unter Typ II Phasenanpassung frequenzverdoppelt. Die Trennung der resultierenden Strahlungen von $1064 \mathrm{~nm}$ und $532 \mathrm{~nm}$ erfolgt über zwei Dichroite. Mit der 532 nm-Strahlung wird der Farbstofflaser (LAS: LDL 20505) gepumpt.

Dieser benutzt das Oszillatorkonzept des streifenden Einfalls (near grazing incidence oder Littman-Anordnung), woraus Laserstrahlung mit vertikaler Polarisation resultiert. Die Oszillatorküvette wird mit einem zweiten Durchgang zusätzlich als transversal gepumpter Vorverstärker genutzt. Als Hauptverstärkerstufe kommt eine Bethune-Zelle (Kapillardurchmesser $3 \mathrm{~mm}$ ) zum Einsatz [Bet81], durch die im mittleren Fernfeld ein nahezu gaußförmi- 


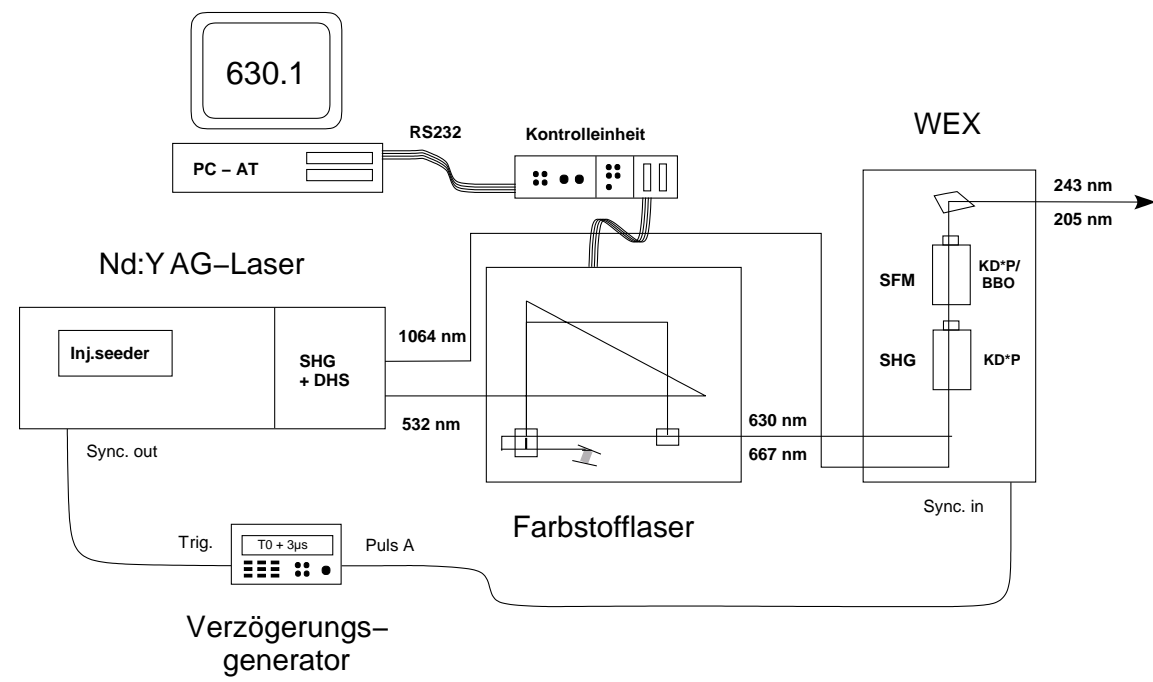

Abbildung 2.4: Aufbau des abstimmbaren Lasersystems bestehend aus ND:YAGLaser, Farbstofflaser und durch Nd:YAG-Laser gesteuerten Wavelength Extender (WEX)

ges Strahlprofil erreicht wird. Der Farbstofflaser erzeugt Licht mit einer Wellenlänge von $630.1 \mathrm{~nm}$. Als Farbstoff kommt in Methanal gelöstes DCM zum Einsatz. Die Konzentration in der Osziallatorküvette beträgt $0.34 \mathrm{~g} / \mathrm{l}$. Für den Hauptverstärker wird die Lösung im Verhältnis 3:1 mit Methanol verdünnt. Im Anschluß an den Farbstofflaser wird sein Strahlprofil mit einem Teleskop auf einen Durchmesser von $6 \mathrm{~mm}$ vergrößert, um in den nachfolgenden nichtlinearen Frequenzkonversionen die Kristalle nicht zu beschädigen.

Die Frequenzkonversion in den UV-Wellenlängenbereich findet im Wavelength Extender (Spectra Physics: WEX-2C) statt (s. Abb. 2.4). Dieser Wavelength Extender stellt eine für die Durchführung von nichtlinearen Prozessen optimierte Umgebung dar, in der in einem staubgeschützten Gehäuse die drei Bereiche (Anordnung zur Zusammenführung der benötigten Laserstrahlen, Aufnahme der nichtlinearen Kristalle samt Kompensatoren für den Ausgleich des Strahlversatzes mit entsprechender Justagevorrichtung und Anordnung der danach notwendigen Wellenlängenseparation durch ein Pellin-Broca-Prisma) untergebracht sind. Bei der Erzeugung von $243 \mathrm{~nm}$ kommen zwei KD*P-Kristalle zum Einsatz: Zunächst wird die vom Farbstofflaser emittierte Strahlung von $630.1 \mathrm{~nm}$ in einem KD*P-Kristall frequenzverdoppelt. Hier wird ein Kristall vom Typ I benutzt, der gemäß der Einteilung in die Typen I und II, d. h. eine Einkopplung der zu mischenden Strahlen unter paralleler bzw. 
senkrechter Polarisation, die praktikable Lösung für die Frequenzverdopplung einer einzigen beteiligten Wellenlänge darstellt. Die für diesen negativen Kristall $\left(n_{o}>n_{e}\right)$ resultierende „ooe"-Phasenanpassung (franz. o: ordinaire, e: extraordinaire) führt zu einer horizontalen Polarisation für die zweite Harmonische.

Diese Strahlung von $315.0 \mathrm{~nm}$ wird nun im zweiten KD*P-Kristall, ebenfalls vom Typ I, mit der Fundamentalen des Nd:YAG-Lasers gemischt. Dazu wird die Polarisationsrichtung des Infraroten durch ein $\lambda / 2$-Plättchen am Eingang des Wavelength Extender in die Horizontale gedreht. Die resultierende UV-Strahlung von $243 \mathrm{~nm}$ wird nach Austritt aus dem zweiten Kristallmodul durch ein Pellin-Broca-Prisma von den anderen Wellenlängen getrennt und steht in vertikaler Polarisation für die Einkopplung in die TOF-Kammer zur Verfügung. In den hier durchgeführten Experimenten lieferte das System in dieser Anordnung durchschnittliche Pulsenergien von $3 \mathrm{~mJ}$.

Nach Verlassen des WEX wird der Strahl über Reflexionen an Prismen an die Flugzeitmassenspektrometerkammer geführt. Hier befindet sich in einer XYZVerschiebekonstruktion eine $400 \mathrm{~mm}$ Quarzlinse, die der Fokussierung des Strahls in das Zentrum des Flugzeitmassenspektrometers dient.

\subsubsection{ArF $/ F_{2}$-Excimer-Laser}

Der in früheren Messungen eingesetzte Excimerlaser (Lambda: COMPex 102) [Nah98, Bau99b] wurde durch ein neueres Modell (Lambda: LPF202) ersetzt. Dieser zeichnet sich durch deutlich höhere Pulsenergien, die spezielle Eignung für fluorhaltige Excimere und geringere zeitliche Puls-zu-Puls Schwankungen aus.

Bei einer Wellenlänge von $193 \mathrm{~nm}$ wird er in diesen Experimenten zur Dissoziation von $\mathrm{HCl}$ benutzt. Die zeitliche Ansteuerung der Gasentladung geschieht über den Nd:YAG Laser (Lasersystem zur lonisation) und einen Verzögerungsgenerator (Stanford Research: DG535). Somit arbeitet er wie das oben beschriebene Lasersystem bei einer Pulsfrequenz von $10 \mathrm{~Hz}$. Das Gerät ist für maximal $20 \mathrm{~Hz}$ ausgelegt. Weitere Daten sind Tab. $2.2 \mathrm{zu}$ entnehmen.

Licht einer Wellenlänge von $193 \mathrm{~nm}$ ist in der Lage Sauerstoff zu dissoziieren. Die freien Radikale bilden an Luft mit den übrigen Sauerstoffmolekülen Ozon. Dadurch wird an Luft ein großer Teil des Laserlichts absorbiert (ca. 20\%). Im übrigen ist die starke Ozonbildung den Experimentatoren im Labor aus gesundheitlichen Gründen nicht zuzumuten. Deswegen wird der komplette Strahlengang vom Excimerlaser bis zur Einkopplung in die TOF-Kammer evakuiert. Der Aufbau ist in Abb. 2.5 dargestellt. 


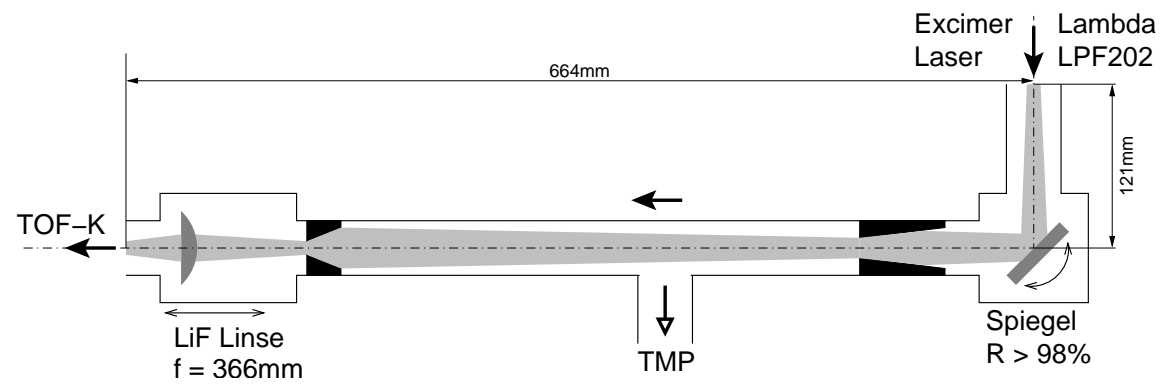

Abbildung 2.5: Gekapselte Strahlführung der $193 \mathrm{~nm}$ Strahlung vom Excimer Laser bis zum Eingang TOF-Kammer. Der Strahlengang kann wahlweise evakuiert oder mit $\mathrm{Ar} / \mathrm{N}_{2}$ gespühlt werden. Zum Evakuieren dient eine Turbomolekularpumpe (TMP) von Varian (Turbo V-60,60 I/s). Die Divergenz der Laserstrahlung wird durch Blenden ausgeglichen. Die Linse und der Spiegel können über Vakuumdurchführungen von außen justiert werden.

Das Rohrsystem kann entweder mit einer Turbomolekularpumpe (Varian: Turbo V-60) auf einen Restgasdruck von $10^{-4}$ mbar evakuiert werden oder mit Stickstoff- oder Argon gespült werden. Auf den ersten Blick erweist sich die Evakuierung des Strahlengangs als die kostengünstigere und auch effektivere Lösung, weil praktisch keine Teilchen mehr vorhanden sind, die Licht absorbieren. Man muß sich allerdings vergegenwärtigen, daß Licht mit einer Wellenlänge von $193 \mathrm{~nm}$ in der Lage ist, die meisten Moleküle zu ionisieren. Diese lonen besitzen in einem evakuierten Strahlengang eine große mittlere freie Weglänge (hier ca. $2 \mathrm{~m}$ ). Somit können sie mit hohen Geschwindigkeiten auf die Optiken prallen und dort die Beschichtungen zerstören. Bei typischem Laboreinsatz sind bei evakuiertem Strahlengang die Beschichtungen auf den Spiegeln und Linsen nach einigen Wochen zerstört. Im Vergleich dazu ist der Einsatz von Stickstoff zum Spülen vergleichsweise kostengünstig. Die lonenbildung findet zwar ebenfalls statt. Durch den leicht über Atmosphäre liegenden Druck ist die mittlere freie Weglänge der lonen jedoch so klein (Bereich Millimeter), daß die Ionen rekombinieren und somit die Optiken nicht beschädigt werden. Der Energieverlust gegenüber einem evakuierten Strahlengang ist in diesem Experiment kleiner als $3 \%$. In dieser Arbeit kommt deswegen ein stickstoffgespülter Strahlengang zum Einsatz. Anders ist die Situation bei Umstellung der Wellenlänge auf $157 \mathrm{~nm}$. Hier absorbiert Stickstoff so stark, daß es als Spülgas ausscheidet. Argon ist bei dem nötigen Gasdurchsatz verhältnismäßig teuer und weist in diesem Bereich ebenfalls leichte Absorption auf. Die Frage nach einem evakuierten oder gespülten Strahlengang ist hier auch in großtechnischen Anwendungen (Lithographie) noch nicht endgültig geklärt. 
Der Strahl besitzt durch die Geometrie der Laserentladungsröhre eine Rechteckform $(26 \times 8 \mathrm{~mm})$. Er wird mit einem rechteckigen, beschichteten Spiegel $(54 \times 27 \mathrm{~mm}, \mathrm{R}>98 \%)$ umgelenkt, der über eine Vakuumdurchführung sowohl um seine vertikale als auch seine horizontale Achse verkippt werden kann. Als weitere Justagemöglichkeit läßt er sich in Richtung des Lasers verschieben. Der stark divergente Laserstrahl $(2 \times 6 \mathrm{mrad})$ wird durch kegelförmige Blenden auf einen Durchmesser von $10 \mathrm{~mm}$ reduziert. Dies geht mit einem starken Leistungsverlust einher, so daß man in Zukunft ein Teleskopsystem mit Zylinderlinsen zur räumlichen Strahlbegrenzung in Erwägung ziehen sollte. Der Strahl wird mit einer unbeschichteten LiF-Linse $(T=88 \%, f=366 \mathrm{~mm})$ in das Zentrum der TOF-Kammer fokussiert.

Für die meisten Messungen wird der Laserstrahl nicht polarisiert, wobei „unpolarisiert" in diesem Fall bedeutet, daß es eine leichte Vorzugspolarisation in vertikaler Richtung gibt [Nah98]. Der Aufbau zur Polarisierung und Polarisationsdrehung des Laserstrahls wird in Abschnitt 2.2.3 behandelt.

\subsubsection{Polarisierung und Einkopplung der Laserstrahlen}

Für die Messung der Anisotropie der Fragmentverteilungen ist eine Drehung der Laserpolarisationsebene notwendig. In früheren Messungen zur genauen Ermittlung der Anisotropiefaktoren von reinen Molekülen, die zur Eichung der Apparatur und der Auswertung benutzt wurden, kam zur Polarisationsdrehung ein Doppelfresnelrhombus für die $243 \mathrm{~nm}$ und ein drehbarer Dünnschichtplattenpolarisator für die $193 \mathrm{~nm}$ Strahlung zum Einsatz. Beide Aufbauten erlauben eine Drehung um beliebige Winkel [Bau99b].

In den Clusterexperimenten dieser Arbeit genügt unter Berücksichtigung der früheren Ergebnisse die Verwendung von zwei Laserpolarisationen, wozu sich die Extremwerte $0^{\circ}$ und $90^{\circ}$ anbieten. Im folgenden wird die Polarisierung beider Lasersysteme beschrieben.

\section{$243 \mathrm{~nm}$}

Die Laserstrahlung von $243 \mathrm{~nm}$ aus dem Nd:YAG-Farbstofflaser-Sytem muß bis zur Einkopplung durch Reflexionsprismen sowohl in der x-y-Ebene als auch in der Höhe manipuliert werden. Eine Polarisationsdrehung erreicht man durch die geeignete Wahl des Strahlengangs und die Anzahl von Reflexionen. Dabei läßt sich die Tatsache ausnutzen, daß eine Drehung der Polarisation um $90^{\circ}$ stattfindet, wenn ein Strahl drei senkrecht zueinander angeordnete Reflexionen erfährt. Die bisherige Anordnung der Einkopplung in das TOFMS behielt die ursprüngliche vertikale Ausrichtung der Polarisation bei, so daß es nur noch 
erforderlich war, einen zweiten Strahlengang mit einem weiteren Prisma (s. Abb. 2.6) aufzubauen.

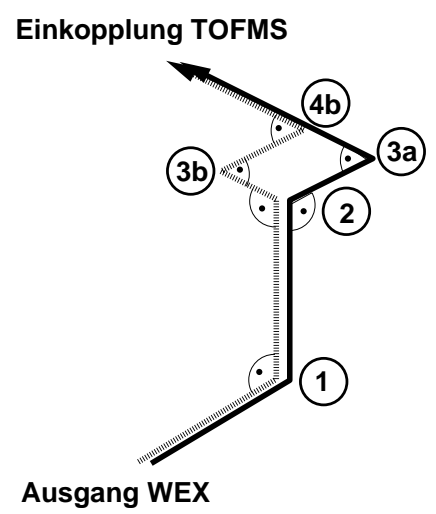

Abbildung 2.6: Einkopplungsaufbau für Polarisationen $0^{\circ}$ (Prismen: 1, 2, 3a) und $90^{\circ}$ (Prismen: 1, 2, 3b, 4b)

Prisma 2 in Abb. 2.6 ist in einer drehbaren Halterung angebracht, die über Anschläge die Einstellung von zwei Positionen unter $90^{\circ}$ zueinander zuläßt. Um den $90^{\circ}$-Strahlengang zu vervollständigen, ist dann nur noch Prisma $4 b$ in seine Halterung einzusetzen. So ist der Wechsel zwischen den Strahlengängen einfach zu bewerkstelligen.

\section{$193 \mathrm{~nm}$}

Der Excimerlaser emittiert bauartbedingt unpolarisiertes Licht. Die Polarisation erfolgt mit einem Dünnschichtplattenpolarisator (TFP: thin film polarizer) aus SQ1 (Quarzglasart) unter einem Winkel von $62^{\circ}$ (aufgrund der Beschichtung größer als Brewsterwinkel) zum Einsatz. Die horizontal zur Einfallsebene polarisierte Strahlung wird transmittiert, die vertikale Polarisation wird vom TFP reflektiert ${ }^{3}$. Wenn man nun die in Abbildung 2.7 gezeigte Anordnung um die Strahlachse rotiert (TFP steht immer unter $62^{\circ}$ zum Strahl), kann man jede beliebige Richtung linear polarisierten Lichtes erzeugen. Der durch den TFP verursachte Strahlversatz wird durch ein einfaches Fenster mit identischer Geometrie ausgeglichen (SQ1, $=2^{\prime \prime}, d=1 / 8^{\prime \prime}, \mathrm{T}>85 \%$ ). Näheres zu dieser Anordnung und zu den Eigenschaften des TFP ist in [Nah98] nachzulesen.

\footnotetext{
${ }^{3}$ Als Definition für vertikale und horizontale Polarisationsrichtung gilt die Ausrichtung relativ zur Ebene, die vom einfallenden und reflektierten Strahl aufgespannt wird.
} 


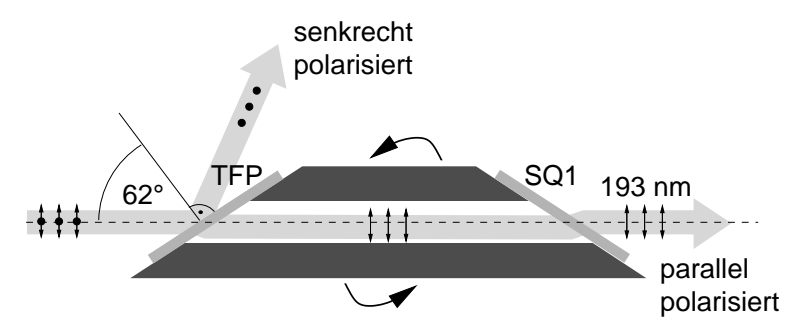

Abbildung 2.7: Polarisationsdreher für $193 \mathrm{~nm}$ mit einem Dünnschichtplattenpolarisator (TFP) zur Polarisierung und einem Fenster aus SQ1 zum Ausgleich des Strahlversatzes. Die Polarisationsebene ist im Verhältnis zur Detektorachse angegeben.

\section{Einkopplung und Bestimmung der Pulsenergien}

Wie oben beschrieben werden die Laserstrahlen über Linsen in das Zentrum des Flugzeitmassenspektrometers fokussiert. Die Einkoppelfenster an der TOF-Kammer sind je nach verwendeter Wellenlänge aus LiF oder Quarz. Für jede Einkopplung gibt es auf der anderen Seite auch eine Auskopplung, was vor allen Dingen nötig ist, um das Streulicht in der Kammer zu reduzieren (s. Abschnitt 2.3.3).

Insbesondere für die Messungen zur Ausrichtung von Molekülen in Laserstrahlen ist es von besonderer Wichtigkeit, die exakte Leistungsdichte der Laser im Zentrum des WMTOFMS zu kennen. An dieser Position im Ultrahochvakuum (UHV) ist eine direkte Messung allerdings nicht möglich. Wenn die Absorption der verwendeten Optiken (Ein- und Auskoppelfenster, Linsen, Prismen) bestimmt worden ist, ist die Messung der Pulsenergie an nahezu jeder beliebigen Position im Strahlengang möglich. Aus praktischen Gründen wird die Pulsenergie der $243 \mathrm{~nm}$ hinter dem WEX, vor dem Passieren der drei/vier Prismen, der Linse und dem Einkoppelfenster gemessen. Die Analyse der $193 \mathrm{~nm}$ Strahlung geschieht dagegen erst hinter dem Auskoppelfentser, so daß hier die Absorption einer zusätzlichen Optik das Ergebnis verfälscht. Für die Bestimmung der Leistungsdichte $I_{L}$ spielen im weiteren die Strahlquerschnitte im TOF-Zentrum sowie die Pulslängen der Laser eine Rolle:

$$
I_{L}=\frac{P_{L}}{F}=\frac{E_{L}^{m e s s} \cdot k / \tau_{L}}{\pi \cdot w_{0}^{2}}
$$

mit $I_{L} \quad$ : Leistungsdichte des Lasers

$P_{L} \quad$ : Leistung des Lasers

$F \quad:$ Fläche des Laserstrahls im Fokus

$E_{L}^{m e s s}$ : gemessene Pulsenergie 


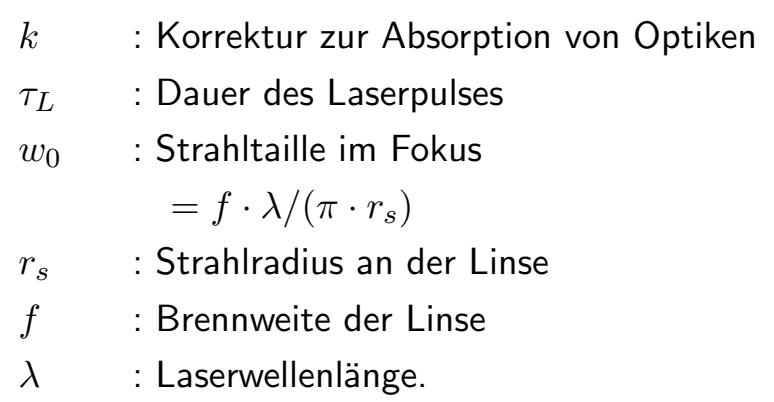

Die Korrekturfaktoren für die benutzten Wellenlängen betragen: $k_{243 n m}=0.44$, $k_{193 n m}=1.69$. Die typischen Leistungsdichten in diesen Experimenten bewegen sich im Bereich von $10^{11} \mathrm{~W} / \mathrm{cm}^{2}$. Die genauen Daten sind bei den jeweiligen Messungen aufgeführt.

\subsection{Wiley-McLaren Flugzeitmassenspektrometer (WMTOFMS)}

In diesem Abschnitt werden die unterschiedlichen Wiley-McLaren Flugzeitmassenspektrometer erläutert, die in dieser Arbeit zum Einsatz kommen. Zuerst wird das WMTOFMS, daß schon in früheren Arbeiten verwendet wurde [Bau99a, Nah98, Fri97] erläutert, anschließend das WMTOFMS mit identischer Geometrie aber einem geschalteten Abzugsfeld zur Orientierung von Molekülen und am Ende des Abschnitts ein WMTOFMS in längerer Ausführung zur Verbesserung des Auf|lösungsvermögens. Dieses WMTOFMS ist zusätzlich gegen elektronische Störeinflüsse und Streulicht abgeschirmt.

\subsubsection{WMTOFMS mit kurzer Driftstrecke}

Abbildung 2.8 zeigt den Aufbau des verwendeten Flugzeitmassenspektrometers, bei dem es sich um ein zweistufiges Modell vom Wiley-McLaren-Typ handelt [Wil55].

Der in unseren Werkstätten gefertigte Eigenbau hat eine einfache Umsetzung des Konstruktionsprinzips zum Ziel. So werden die Flugstrecken durch im Quadrat angeordnete Edelstahlstangen gebildet, an deren Beginn und Ende sich Edelstahlblenden mit einem Öffnungsdurchmesser von $25 \mathrm{~mm}$ befinden. Um Feldausbrüchen vorzubeugen sind die kreisförmigen Blendenöffnungen mit einem hochtransmittiven Nickelnetz (70LPI Nickel Mesh, Buckbee-Mears, 85\% Transmission) versehen. Der lonendetektor wird durch Mikrokanalplatten in Chevron-Anordnung gebildet, der bei einer Betriebspannung von $2 \mathrm{kV}$ einen Verstärkungsfaktor von $10^{6}$ besitzt. Die Signalaufnahme und -speicherung erfolgt über ein 
Digitales Speicheroszillokop (DSO, Tektronix TDS 520, 500 MHz 500 MSamples), wobei das Startsignal für die Messung von der ansteigenden Flanke des Laserpulses, bestimmt durch eine schnelle Photodiode, gegeben wird. Damit liegt die maximale und auch in diesen Messungen benutzte Zeitauflösung bei 2 ns.

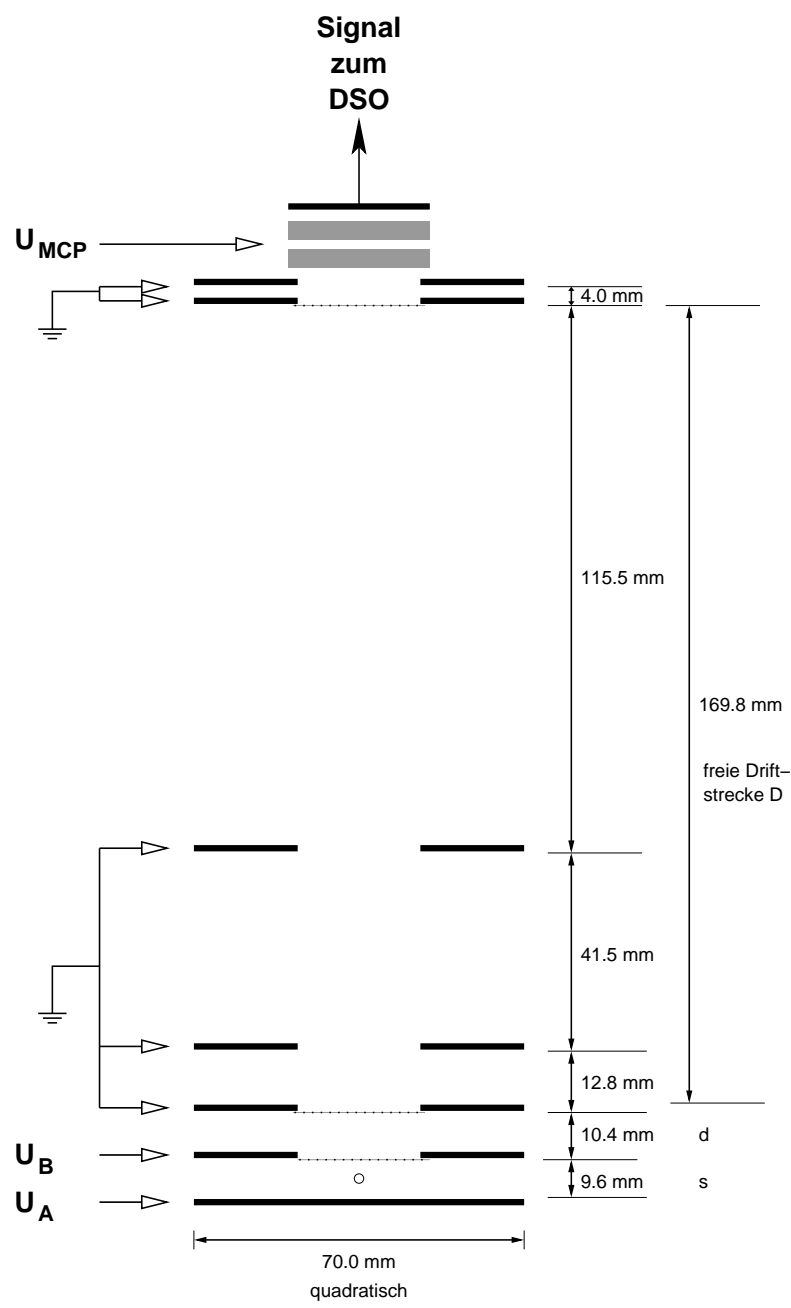

Abbildung 2.8: Aufbau des Flugzeitmassenspektrometers in kurzer, nicht abgeschirmter Ausführung: $\circ$ : lonisationsort; $U_{A}$ : Abzugsspannung; $U_{B}$ : Beschleunigungsspannung; $U_{M C P}$ : Versorgungsspannung für Multi-Channel-Plates; DSO : Digitales Speicheroszilloskop 
Für die Spannungsversorgung der statischen Felder $U_{A}$ und $U_{B}$ kommen Netzgeräte der Firma Rohde \& Schwarz vom Typ NGM 280/0.1 zum Einsatz. Dabei gilt für die übliche Beschreibung der Spannungen, wie sie in Abschnitt 1.3.1 erfolgt: Abzugsspannung $U_{s}=$ $U_{A}-U_{B}$ und Beschleunigungsspannung $U_{d}=U_{B}$. Zur Erfüllung der Ortsfokusbedingung erster Ordnung läßt sich das Verhältnis zwischen den Spannungen für diese TOF-Geometrie zu $U_{d}=3.46 \cdot U_{s}$ bestimmen.

Der MCP-Detektor wird mit einem Hochspannungsnetzgerät (FUG HCN 14-6 500) betrieben. Die elektrischen Anschlüsse und die Spannungsteilung für die einzelnen Platten des MCP sind in Abb. 2.9 skizziert. Eine nähere Erläuterung des Flugzeitmassenspektrometers, des Detektors und der Datenaufnahme mittels DSO befindet sich in [Fri97].

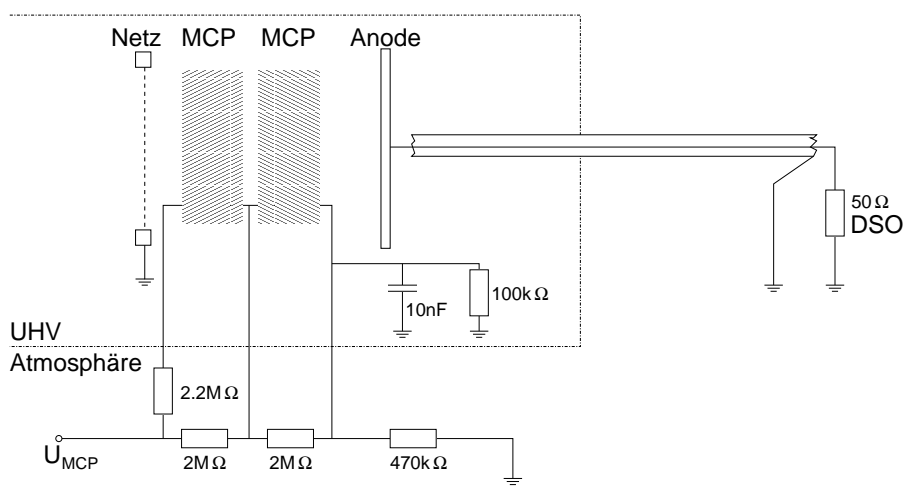

\begin{abstract}
Abbildung 2.9: Aufbau und Spannungsversorgung des lonendetektors (MCP) mit Signalauskopplung in das digitale Speicheroszilloskop. Dabei bezeichnet UHV das Innere der TOF-Kammer, in dem Ultrohochvakuum herrscht und Atmosphäre das Labor außerhalb der Vakuumapparatur.
\end{abstract}

\title{
2.3.2 WMTOFMS mit geschaltetem Abzugsfeld
}

In diesen Experimenten sollen die in Abschnitt 1.4 erwähnten Moleküle HRgX im Laserfeld ausgerichtet und im elektrostatischen Feld im WMTOFMS orientiert werden. Die Orientierung in schwachen elektrostatischen Feldern, wie sie hier im Niedrigfeldmodus herrschen, setzt einen hohen Grad der Ausrichtung durch den Laser voraus. Für die Parameter im WMTOFMS ist das elektrische Feld im Abzugsbereich von Interesse, sprich der Plattenabstand $s$ (fest) und die Abzugsspannung $U_{S}$ (variabel). Zum einen spielt natürlich die Höhe der Spannung eine Rolle für die Orientierung, was ausführlich in Abschnitt 1.4.2 behan- 
delt wird. Zum anderen ist aber die Richtung des elektrischen Feldes von entscheidender Bedeutung für die Orientierung der Moleküle.

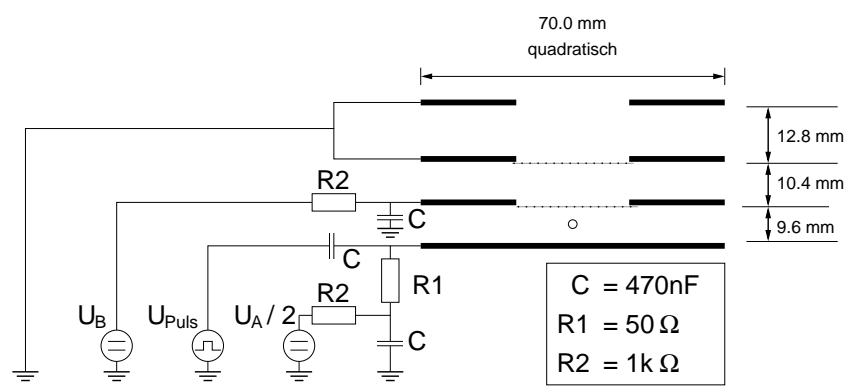

Abbildung 2.10: Beschaltung der elektischen Felder im WMTOFMS mit den festen Spannungen $U_{A} / 2$ und $U_{B}$ sowie der gepulsten Spannung $U_{P u l s}$, die, wenn sie eingeschaltet ist, vom Betrag identisch ist mit $U_{A} / 2$.

Wenn sich die Feldrichtung und damit die Orientierungsrichtung der Moleküle ändert, ändert sich natürlich auch die Flugrichtung der lonen. In diesem Experiment werden normalerweise Flugzeitspektren von Wasserstoffionen (positiv) gemessen. Die Änderung der Flugrichtung führt allerdings dazu, daß die lonen auf der untersten Platte aufschlagen und nicht zum Detektor fliegen. Damit wäre die Meßmethode ad absurdum geführt. Es muß eine zeitliche Änderung der Feldrichtung erfolgen, wofür die Spannung im Laufe eines Meßereignisses im zeitlichen Bereich von einigen Nanosekunden umgeschaltet werden muß. Wie diese Schaltung im WMTOFMS aussieht, ist in Abb. 2.10 skizziert. Die zeitlichen Abfolgen, Spannungen und Richtungen des Abzugsfeldes sind in Abb. 2.11 erläutert.

Der Meßprozeß läßt sich qualitativ folgendermaßen zeitlich einteilen (eingekreiste Nummern s. Abb. 2.11):

\section{Das Abzugsfeld zeigt entgegengesetzt zur Detektorrichtung. (2)}

2. Der Laserpuls erreicht das TOF-Zentrum. (1)

a) Das Molekül wird ausgerichtet, so daß das Wasserstoffatom vom Detektor weg zeigt.

b) Das Molekül wird dissoziiert.

c) Das Wasserstoffatom wird mittels REMPI ionisiert.

3. Das Wasserstoffion startet seine Flugbahn und entfernt sich vom Detektor. 


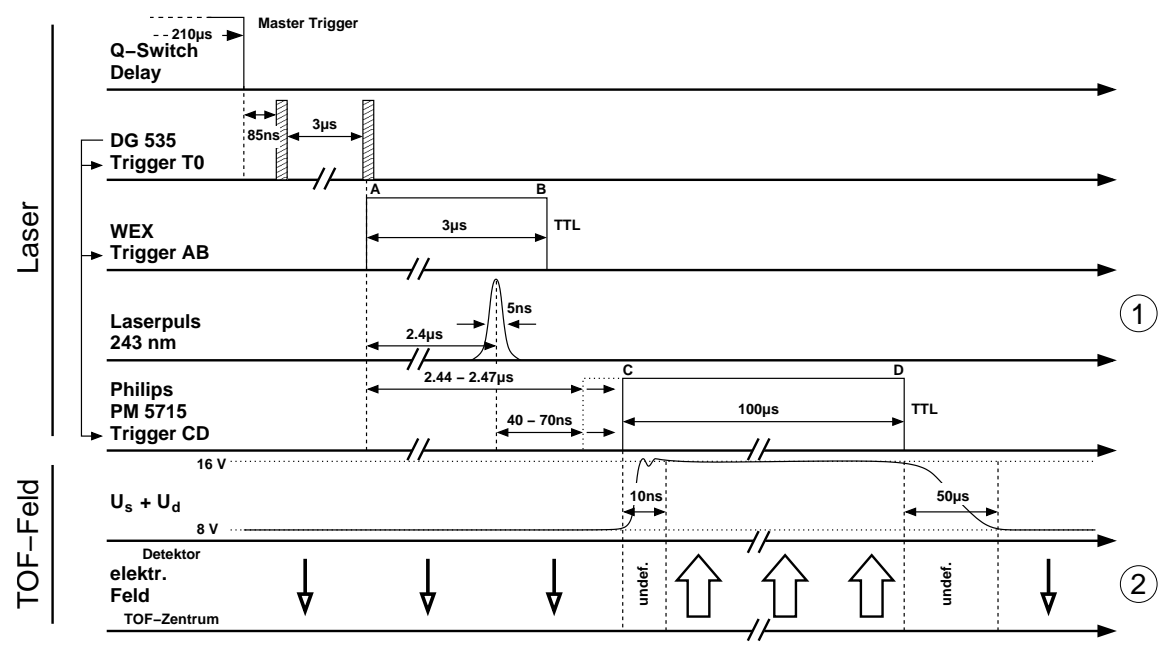

Abbildung 2.11: Zeitliche Pulsfolge des Dissoziationslasers und des elektrischen Feldes im WMTOFMS.

4. Der Laserpuls ist beendet. (1)

5. Das elektrische Feld wird in Detektorrichtung umgeschaltet. (2)

6. Das Wasserstoffion wird abgebremst und fliegt in Detektorrichtung.

7. Nachweis des Wasserstoffions am Detektor.

8. Das elektrische Feld wird wieder entgegen der Detektorrichtung geschaltet. (2)

Die Umschaltung des elektrischen Feldes geschieht, indem der Gleichspannung $U_{A}$ ein HF-Feld überlagert wird. Unter Verwendung eines Verzögerungsgenerators (Stanford Research: DG535) und eines Pulsgenerators (Philips PM5715) gelingt das Umschalten des Feldes in einem Zeitfenster von $10 \mathrm{~ns}$. Als Master Trigger dient der Laserpuls des Nd:YAG Lasers. Das elektrische Feld zeigt für die Dauer von $100 \mu \mathrm{s}$ in Detektorrichtung. Die Gesamtflugzeit der Wassersoffionen beträgt selbst bei schwachen Feldern maximal $6 \mu \mathrm{s}$.

Ein schnelleres Umschalten des elektrischen Feldes in weniger als 1 ns ist mit BehlkeSchaltern möglich. Dies brächte den Vorteil mit sich, daß die Felder sich für einen kürzeren Zeitraum in einem undefinierten Zustand befänden. In unserem Aufbau gestaltet sich 
die Messung der elektrischen Spannungen als nahezu unmöglich. Durch die RC-Glieder (s. Abb. 2.10) und die überlagerte HF-Spannung lassen sich die außerhalb der TOFKammer gemessenen Spannungen nicht direkt auf die an den Platten des WMTOFMS anliegenden Spannungen übertragen. Es ist nur eine ungefähre Abschätzung möglich. Dies ist von Bedeutung für die Auswertung in dem später beschriebenen Trajektoriensimulationsprogramm (s. Abschnitt 3.2.1).

\subsubsection{WMTOFMS mit langer abgeschirmter Driftstrecke}

In späteren Messungen dieser Arbeit kam ein optimiertes WMTOFMS zum Einsatz. Dieses ist in Abb. 2.12 dargestellt. Es ist bisher nur für Gleichspannungen eingesetzt worden.

Gegnüber älteren Ausführungen in unserer Gruppe unterscheidet es sich in mehreren Punkten:

1. Runde Plattengeometrie. Aus dieser folgen homogenere Felder.

2. Durch ein Edelstahlrohr abgeschirmte Driftstrecke. Der Einfluß äußerer elektrostatischer Einflüsse auf die lonentrajektorien wird minimiert. Es kann nahezu kein Streulicht auf den Detektor fallen.

3. Verlängerung der Driftstrecke. Die Länge der Driftstrecke wurde fast verdoppelt, was zu einer Erhöhung des Auf|lösungsvermögens führt.

4. MSP als Detektor. Die micro sphere plates besitzen bei einer Spannung von $3.5 \mathrm{kV}$ einen Verstärkungsfaktor von $10^{7}$ (MCP: $\left.10^{6}\right)$.

An den Oberflächen der Ein- und Auskoppelfenster der TOF-Kammer wird Laserlicht gestreut. In der alten Anordnung konnte dies durch Mehrfachstreuung ungehindert auf den MCP Detektor treffen. Dieser ist genauso wie ein MSP hochempfindlich für Photonen. In früheren Messungen haben die $193 \mathrm{~nm}$ Photonen aus dem Excimer Laser die Flugzeitspektren durch Übersättigung des MCP zur quantitativen Auswertung nahezu unbrauchbar gemacht [Nah98]. Dazu kommen die elektronischen Einstreuungen durch die HV-Thyratron-Entladung des Excimer Lasers. Beide Einflüsse konnten durch die Abschirmung der Driftstrecke und damit auch des MSP fast vollständig ausgeschaltet werden. Die Flugzeitspektren werden nur im Bereich bis zu etwa $1 \mu$ s beeinflußt, der aber für diese Auswertungen nicht von Belang ist. Die Wasserstofffragmente erreichen je nach eingestellten Spannungen in einer Zeit zwischen 2.5 und $6 \mu$ s den Detektor. 
Zur Erfüllung der Ortsfokusbedingung erster Ordnung läßt sich das Verhältnis zwischen den Spannungen für diese TOF-Geometrie zu $U_{d}=4.78 \cdot U_{s}$ bestimmen.

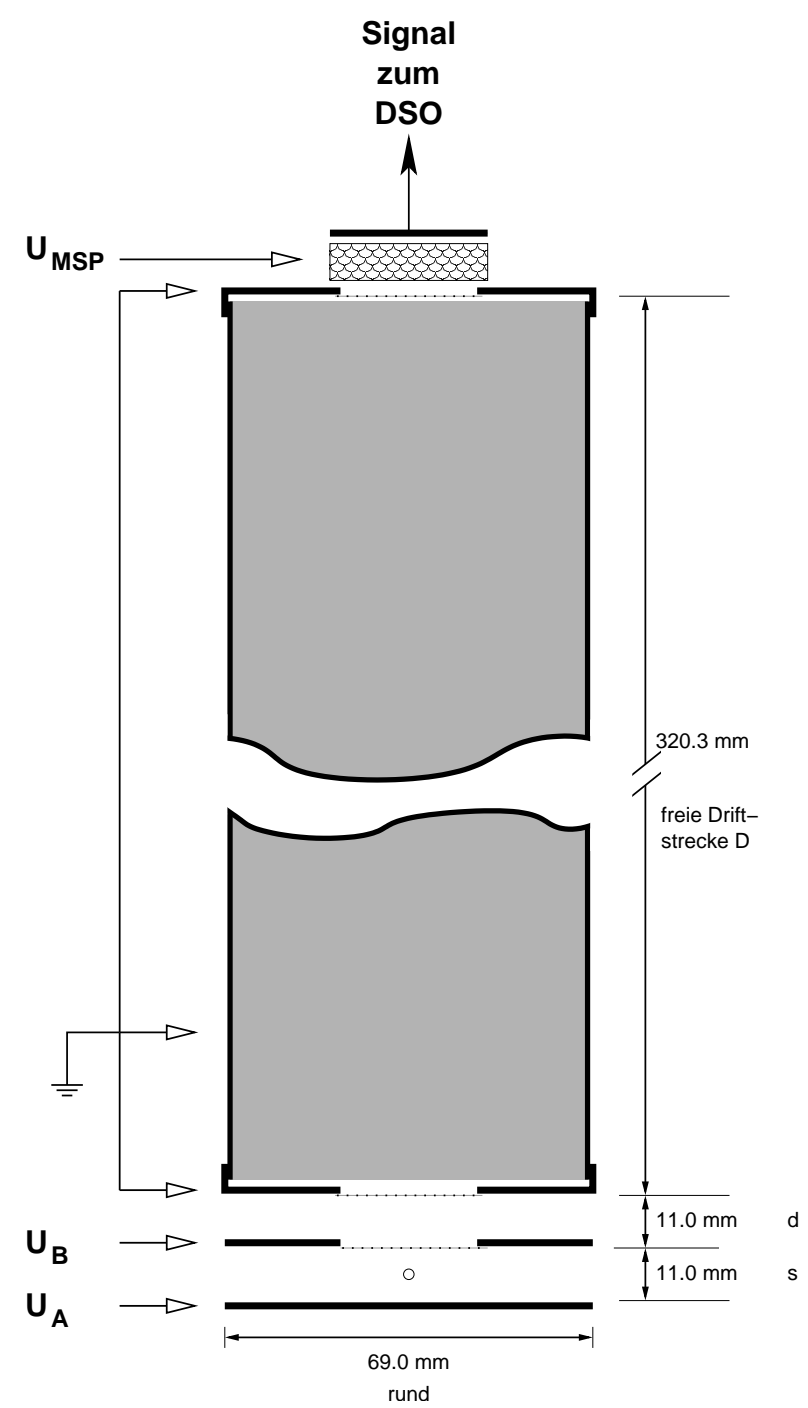

Abbildung 2.12: Aufbau des Flugzeitmassenspektrometers in langer, abgeschirmter Ausführung in runder Grundgeometrie: $\circ$ : lonisationsort; $U_{A}$ : Abzugsspannung; $U_{B}$ : Beschleunigungsspannung; $U_{M S P}$ : Versorgungsspannung für Micro-Sphere-Plates; DSO : Digitales Speicheroszilloskop 
Die Beschaltung des MSP und die geänderte Signalauskopplung sind in Abb. 2.13 skizziert. Die verwendeten Netzgeräte sind identisch mit den in Abschnitt 2.3.1 beschriebenen.

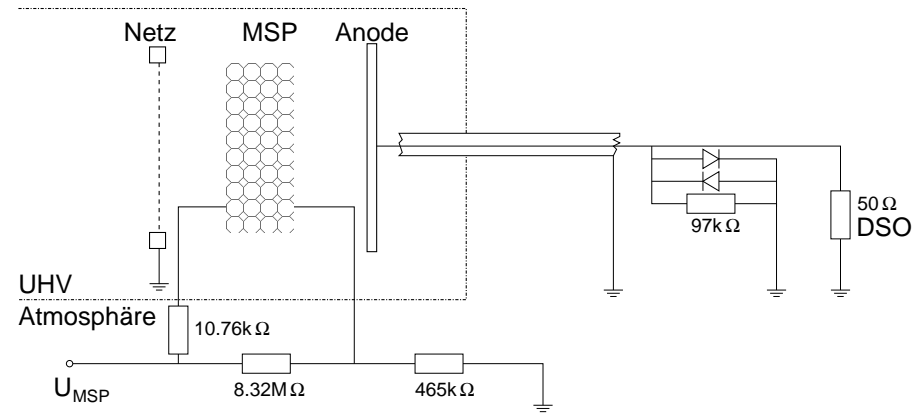

Abbildung 2.13: Aufbau und Spannungsversorgung des lonendetektors (MSP) mit Signalauskopplung in das digitale Speicheroszilloskop.

Exemplarisch ist die Signalverstärkung des MSP für Wasserstoffionen und Streulicht in Abb. 2.14 aufgezeigt. Die Verstärkung nimmt exponentiell mit der angelegten Spannung zu (logarithmische Auftragung). In diesen Experimenten wird in einem Bereich $3.0 \leq$ $U_{M S P} \leq 3.5 \mathrm{kV}$ gearbeitet.

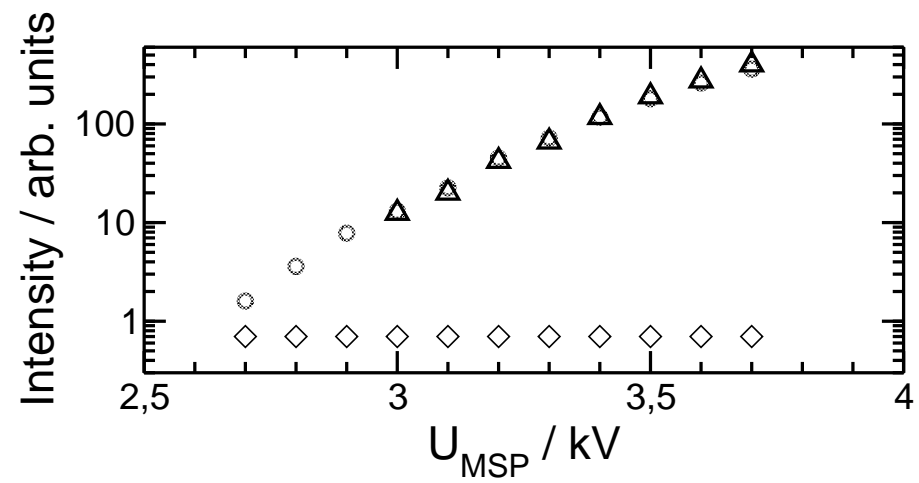

Abbildung 2.14: Die Signalverstärkung der Micro-Sphere-Plates ist für das Rauschen des Detektors $(\diamond)$, für Photonen, resultierend aus Streulicht der Laserstrahlung $(\triangle)$ und für Protonen aus der lonisation von Wasserstoffatomen $(\bigcirc)$ in Abhängigkeit von der MSP-Spannung $\left(U_{M S P}\right)$ aufgetragen. 


\subsubsection{Untergrund-Massenspektrum im Hochfeldmodus}

Trotz des in der TOF-Kammer vorliegenden Drucks von $5 \cdot 10^{-9}$ mbar durch Kühlung mit einem Helium-Kaltkopf ist in den Messungen immer noch ein Untergrundanteil zu verzeichnen, der aus der Photodissoziation von Restgasmolekülen resultiert. Einen großen Anteil des Untergrundes bilden Kohlenwasserstoffe, die aus Pumpenöl und Lagerschmiermitteln stammen. Diese fragmentieren durch Absorption mehrerer Photonen teilweise vollständig ${ }^{4}$, was zu einer Erhöhung der Konzentration an Wasserstofffragmenten führt.
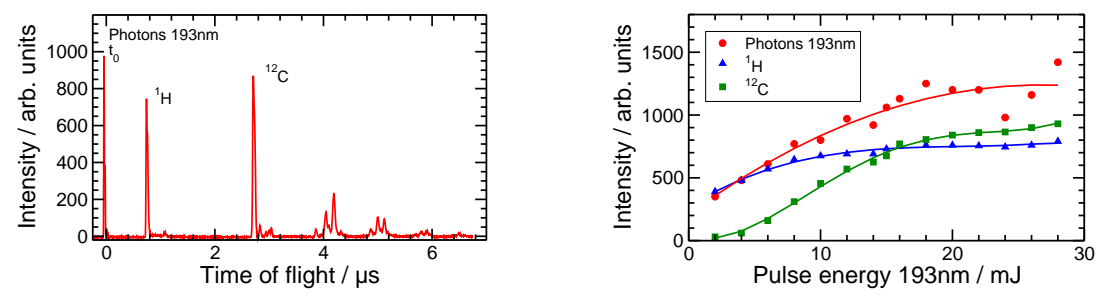

Abbildung 2.15: Massenspektrum im Hochfeld-Modus mit $193 \mathrm{~nm}$ als Dissoziationsund lonisationswellenlänge

In Abbildung 2.15 ist ein im Hochfeldmodus $\left(\mathrm{U}_{\mathrm{s}}=280 \mathrm{~V}, \mathrm{U}_{\mathrm{d}}=800 \mathrm{~V}\right)$ aufgenommenes Untergrund-Spektrum dargestellt. Dabei wird mit dem ArF-Laser $(\lambda=193 \mathrm{~nm}$, Pulsenergie bis zu $30 \mathrm{~mJ}$ ) gearbeitet, der sowohl die Moleküle dissoziiert als auch die Fragmente ionisiert (nicht-resonante Mehrphotonenionisation).

Im gezeigten Spektrum ist ein großer Teil der Alkyl-Gruppen von $\mathrm{C}^{+}$bis $\mathrm{C}_{5} \mathrm{H}_{8}^{+}$aufgelöst sichtbar. Im rechten Teil des Bildes ist dargestellt, wie sich die Signale von $\mathrm{H}^{+}$, $\mathrm{C}^{+}$und Photonen in Abhängigkeit von der Laserleistung verändern. Die starke Erhöhung der Laserleistung führt nicht zu einer Erhöhung des Photonensignals, das aus Streulicht herrührt. Dies ist auf die konvergentere Laserstrahlung bei höheren Pulsenergien zurückzuführen (betriebsbedingt). Die Entwicklung der $\mathrm{H}^{+}$und $\mathrm{C}^{+}$Signale spricht dafür, daß es für Pulsenergien von mehr als $20 \mathrm{~mJ}$ gelingt, alle Fragmente zu ionisieren.

\footnotetext{
${ }^{4}$ Kohlenwasserstoffe besitzen im ultravioletten Spektralbereich breite Absorptionsbanden mit großem Querschnitt.
} 



\section{Auswertung und Simulation}

Die zentralen Größen dieser Arbeit sind die Energie- und Winkelverteilungen von Wasserstofffragmenten aus der Photodissoziation von Halogenwasserstoff-Molekülen in Clusterumgebungen. Gemessen werden die Flugzeitspektren der Wasserstofffragmente in einem Wiley-McLaren Flugzeitmassenspektrometer. Dieses wird im Niedrigfeldmodus betrieben, was für diese Messungen folgenden Vorteil bringt: Bei den niedrigen Abzugsspannungen erreichen Fragmente mit einer höheren Startgeschwindigkeit den Detektor früher als die mit kleiner Startgeschwindigkeit. Die direkte Korrelation zwischen Geschwindigkeit und kinetischer Energie erlaubt somit die Messung der Energieverteilung. Zur Ermittlung der Energieverteilungen werden die Flugzeitspektren mittels eines Monte-Carlo (MC) Trajektoriensimulationsprogramms und eines Matrixinversionsverfahrens in den Energieraum transformiert. Dabei spielt insbesondere die Detektionswahrscheinlichkeit im WMTOFMS in Abhängigkeit von der Anfangsgeschwindigkeit der Fragmente eine zentrale Rolle. Diese muß auch zur Ermittlung der Anisotropieparameter aus Flugzeitspektren unter verschiedenen Laserpolarisationen berücksichtigt werden.

Es werden das Meßprinzip im WMTOFMS, die Ermittlung der reinen Signalspektren, die Transformation von Flugzeitspektren in den Energieraum und die verwendeten Programmpakete an Hand von Beispielen und Tests erläutert.

\subsection{Analyse der gemessenen Flugzeitspektren}

\subsubsection{Meßprinzip}

Im folgenden soll hier das Meßprinzip sowie das Aussehen der Flugzeitspektren erläutert werden. $\mathrm{Zu}$ berücksichtigende Größen sind dabei das Übergangsdipolmoment $\vec{\mu}$, dessen Winkel $\theta$ zur Molekülachse, der für ein diatomares Molekül nur die Werte $0^{\circ}$ und $90^{\circ}$ annehmen kann, die Detektorachse, die die Verbindung zwischen Dissoziation im TOF-Zentrum 
und dem Detektor herstellt sowie der Winkel $\alpha$ zwischen linear polarisierter Laserstrahlung und der Detektorachse.
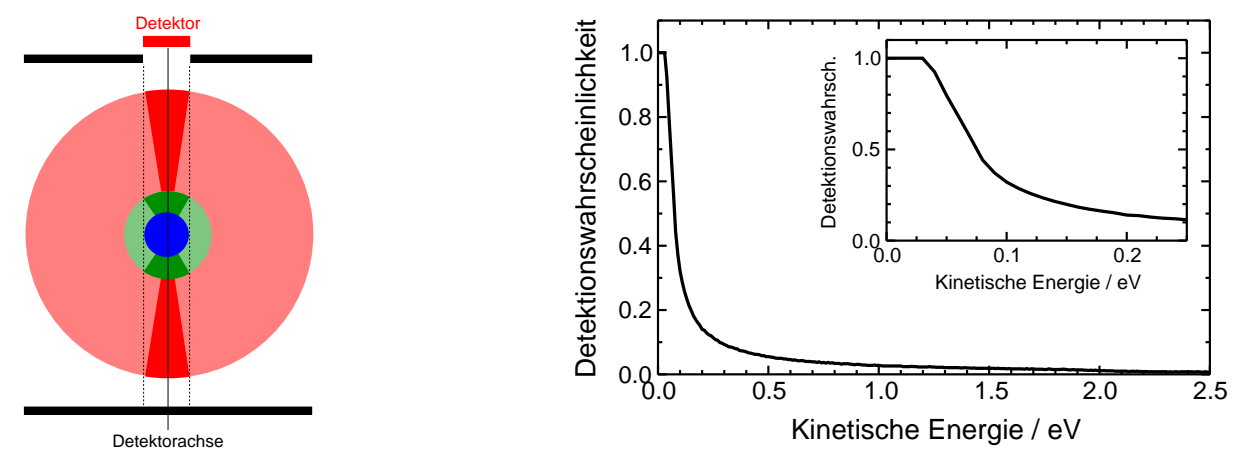

Abbildung 3.1: Detektionswahrscheinlichkeit im WMTOFMS: Die linke Seite zeigt exemplarisch, welche Fragmente mit einer Anfangsgeschwindigkeit (Radius der Kreise) im elektrischen Feld auf den Detektor beschleunigt werden (jeweiliger Kegel in dunklerer Farbe). Die Detektionswahrscheinlichkeit für langsame Fragmente ist höher als für schnelle. Unterhalb einer bestimmten Anfangsgeschwindigkeit (blauer Kreis) werden alle Fragmente nachgewiesen. Quantitativ ist dies im rechten Teil des Bildes gezeigt. Hier ist die Detektionswahrscheinlichkeit in Abhängigkeit von der Anfangsenergie der Fragmente für typische Einstellungen unseres WMTOFMS gezeigt. Für die Berechnung wurde das in Abschnitt 3.2.2 beschriebene Simulationsprogramm benutzt.

Durch die im Verhältnis zum Plattenabstand im Abzugsfeld des WMTOFMS lange Driftstrecke und die relativ kleinen Abzugsfelder arbeitet das Spektrometer unter core sampling Bedingungen. Das bedeutet, daß nur Fragmente mit einer geringen Geschwindigkeitskomponente senkrecht zur Detektorachse am Detektor nachgewiesen werden. Wenn man sich eine isotrope (gleichmäßige) Verteilung der Startrichtung der Fragmente mit einer einheitlichen Geschwindigkeit vorstellt, so schneidet man aus dieser Verteilung ein schmales Segment heraus. Nur Fragmente aus diesem Segment werden nachgewiesen. Hier ist allerdings noch eine wichtige Ergänzung anzuführen. Fragmente die genau in entgegengesetzte Richtung (vom Detektor weg) fliegen, werden zuerst abgebremst und anschließend in Richtung Detektor beschleunigt. Befinden sie sich in einem nahezu identischen Kegel gespiegelt am lonisationsort, werden sie ebenfalls nachgewiesen. Mit der Anfangsgeschwindigkeit verändert sich die Detektionswahrscheinlichkeit. Fragmente ohne Anfangsgeschwindigkeit werden immer nachgewiesen, weil sie sich nicht senkrecht von der Detektorachse wegbe- 
wegen. Fragmente mit extrem hohen Anfangsgeschwindigkeiten werden nur nachgewiesen, wenn der Vektor der Geschwindigkeit nahezu parallel zur Detektorachse ausgerichtet ist. Dieses Prinzip ist in Abb. 3.1 dargestellt, auf der linken Seite anschaulich und rechts als berechnete Detektionswahrscheinlichkeit für unser WMTOFMS.

Die unterschiedlichen Anfangsgeschwindigkeiten und -richtungen prägen das Flugzeitspektrum dahingehend, daß die Fragmente mit der höchsten Anfangsgeschwindigkeit in Detektionsrichtung den Detektor zuerst erreichen. Dem schließen sich Fragmente mit geringerer Anfangsenergie in Detektionsrichtung an. In der Mitte des Spektrums befinden sich die Fragmente ohne Anfangsgeschwindigkeit. Mit späteren Flugzeiten erscheinen nun die Fragmente mit vom Detektor wegzeigenden Anfangsgeschwindigkeiten. Im Prinzip sind sie eine Spiegelung des vorderen Teils an der Position der Fragmente ohne Anfangsgeschwindigkeit. Zuerst erscheinen langsame Fragmente, weil sie im Abzugsfeld schnell abgebremst werden und anschließend in Detektorrichtung beschleunigt werden. Die Fragmente mit hohen kinetischen Anfangsenergien, die vom Detektor weg starten, bilden zeitlich den Abschluß des Spektrums. Ihr Abbremsungsvorgang im Abzugsfeld dauert am längsten.

Neben der Energetik beeinflußt insbesondere die Winkelverteilung der Fragmente die gemessenen Flugzeitspektren: Der Zusammenhang zwischen Laserpolarisation $\alpha$ und der Dissoziation der Moleküle in Abhängigkeit von ihrem Übergangsdipolmoment wird in Abb. 3.2 dargestellt. Hier ist gezeigt, welche Moleküle dissoziiert werden und welche Fragmente aus der Dissoziation dann tatsächlich am Detektor nachgewiesen werden. Die Geschwindigkeiten der Fragmente aus der Dissoziation der freien Moleküle ist so hoch, daß diese, wenn ihre Anfangsgeschwindigkeit senkrecht zur Detektorachse ausgerichtet ist, das WMTOFMS verlassen und nicht nachgewiesen werden können.

Mit einer Laserpolarisation senkrecht zur Detektorachse kann man bei dieser Meßmethode nur Wasserstofffragmente aus der Dissoziation eines Moleküls mit senkrechtem Übergangsdipolmoment nachweisen. Analoges gilt für parallele Polarisation und Übergangsdipolmomente parallel zur Molekülachse. Der Winkel zwischen Molekülachse und Übergangsdipolmoment wird mit $\theta$ bezeichnet ${ }^{1}$. Dieser Nachweis mit einem quasi punktförmigen Detektor führt dazu, daß der Winkel $\alpha$ zwischen Detektorachse und Laserpolarisation eine Projektion auf das Übergangsdipolmoment darstellt. Somit sind hier die Winkel $\alpha$ und $\theta$ identisch.

\footnotetext{
${ }^{1}$ Für ein diatomares Molekül kann er nur die Werte $0^{\circ}$ und $90^{\circ}$ annehmen.
} 


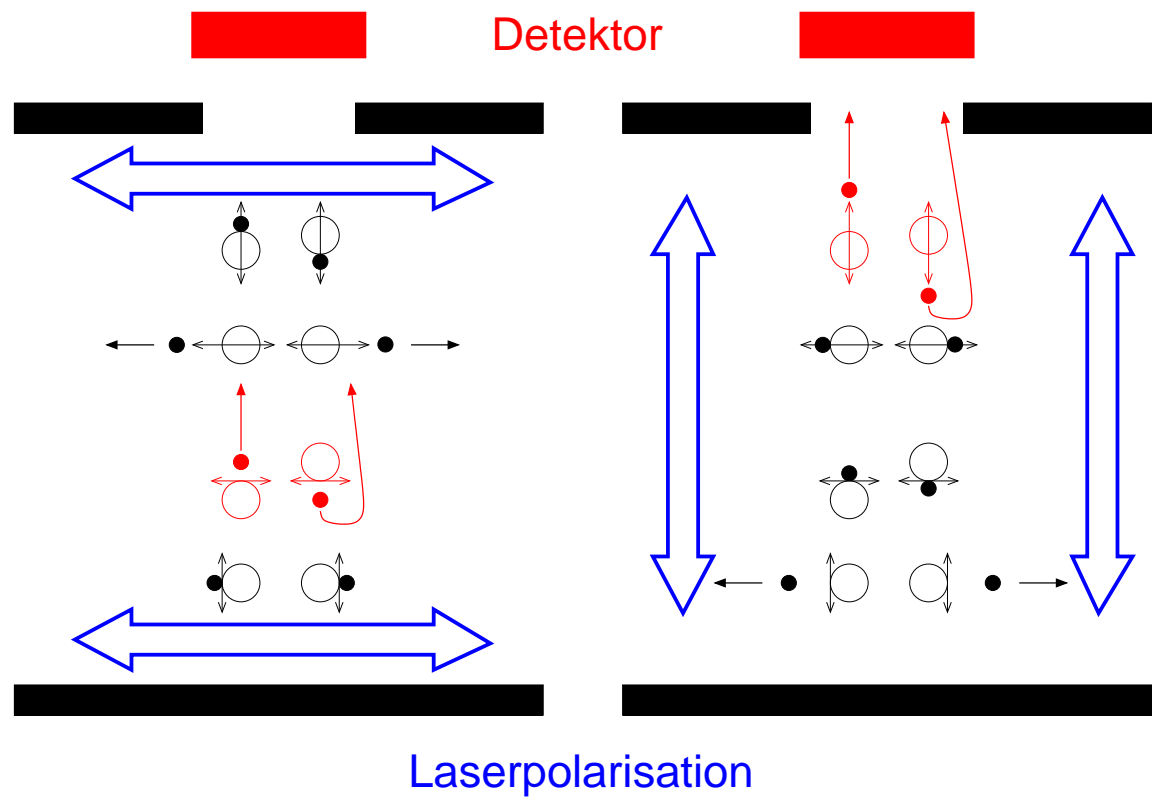

Abbildung 3.2: Gezeigt sind für die Projektion in die Ebene die acht möglichen Extremorientierungen für ein diatomares Molekül, das sowohl ein senkrechtes als auch ein paralleles Übergangsdipolmoment besitzt. Dabei symbolisieren die großen, leeren Kreise das Halogenatom und die kleinen, vollen das Wasserstofffragment. Die Doppelpfeile senkrecht oder parallel zur Molekülachse stehen für das Übergangsdipolmoment. Die Moleküle befinden sich im Abzugsfeld des mit schwarzen Balken gekennzeichneten WMTOFMS (Dissoziationsort). Die blauen Doppelpfeile zeigen die Polarisation des Dissoziationslasers an. Die roten Rechtecke stellen die Detektoren dar. Es wird gezeigt, daß Moleküle mit einem Übergangsdipolmoment parallel zur Laserpolarisation dissoziiert werden, die anderen nicht. Es werden aber nur die Wasserstofffragmente zum Detektor abgezogen, deren Anfangsgeschwindigkeit parallel zur Detektorachse ist (rot gekennzeichnet). Die Fragmente, die zuerst vom Detektor wegfliegen, werden im Abzugsfeld abgebremst und anschließend in Richtung Detektor beschleunigt.

\subsubsection{Ermittlung der Signalspektren}

Wie in allen Experimenten gibt es auch hier Effekte, die die Aufnahme des Signals derart beeinflussen, daß Korrekturen notwendig sind. Ein großer Teil der Korrekturen ist in das MC Trajektoriensimulationsprogramm (Abschnitt 3.2.1) eingebaut. Hier soll deswegen nur der Einfluß von zusätzlich dissoziierten Molekülen und ionisierten Fragmenten aus dem Restgasuntergrund auf die Spektren diskutiert werden. 
Kohlenwasserstoffe aus Diffusionspumpenöl und Schmiermittel und Wasserdampf sind die Quellen für Wasserstoffatome. Diese Moleküle können ebenfalls dissoziiert werden. Die lonisation und der Nachweis der Wasserstofffragmente aus diesen Quellen verfälscht das Nutzsignal. Dazu kommen bei Experimenten mit Pick-up-Präparation freie Halogenwasserstoff-Moleküle, die aus der Selektorkammer in die TOF-Kammer diffundieren und sich auch in den Flugzeitspektren niederschlagen. Ein zusätzlicher Effekt ist Pick-up von Restgasmolekülen durch den Clusterstrahl, der zu einer Erhöhung des Untergrundsignals führt.

Im folgenden ist erläutert, welche Messungen nötig sind, und wie diese arithmetisch miteinander verknüpft werden müssen, um ein reines Signalspektrum zu erhalten.

Messungen bei mittels Pick-up präparierten Clustern:

1. Signal mit allen Untergrundeffekten.

2. Restgasuntergrund in der TOF-Kammer ohne weitere äußere Einflüsse.

3. Untergrund bedingt durch den Molekularstrahl (Pick-up von Restgas).

4. Untergrund durch Diffusion aus der Pick-up-Zelle in die TOF-Kammer.

In all diesen vier Messungen ist der Restgasuntergrund in der TOF-Kammer vertreten. Somit erhält man das Signal durch:

Signal $=1 .-3 .-4 .+2$.

Der Restgasuntergrund (2.) muß einmal addiert werden, weil er sonst doppelt abgezogen wird.

Messungen bei mittels Koexpansion erzeugten Clustern:

1. Signal mit allen Untergrundeffekten.

2. Untergrund bedingt durch den Molekularstrahl (Pick-up von Restgas).

Das Nutzsignal ergibt sich damit zu:

Signal $=1 .-2$.

Weil der Restgasuntergrund der TOF-Kammer in Messung 2. enthalten ist, ist keine weitere Untergrundmessung nötig.

Zur Akquisition der Flugzeitspektren mit dem digitalen Speicheroszilloskop (DSO) wird dessen Mittelungsfunktion genutzt. Ein Flugzeitspektrum aus einem Laserpuls besteht aus 
einigen hundert Ereignissen (Wasserstofffragmente, deren Trajektorien auf den Detektor geführt haben), die noch keine zuverlässige Aussage über die Struktur des Spektrums zulassen. Aus diesem Grund werden im DSO mehrere tausend Einzelspektren (typisch 10000 ) eingelesen und intern gemittelt. Bei einer Laserfrequenz von $10 \mathrm{~Hz}$ beträgt die Meßdauer knapp $17 \mathrm{~min}$.

Auch bei diesen gemittelten Spektren ist insbesondere bei den Untergrundspektren noch deutliches Rauschen erkennbar. Dies liegt an der niedrigen Gesamtintensität der Untergrundspektren, bzw. dem guten Signal-zu-Rausch verhältnis von $R_{S / N}>30$. Aus diesem Grund bietet sich für die Verknüpfung mit der eigentlichen Messung eine Glättung der Untergrundspektren an, um die Einflüsse des Rauschens nicht zu summieren.

Das reine Restgasuntergrundspektrum entspricht einer Gauß-Verteilung, mit der es hier gefittet wird. Das Untergrundspektrum aus dem Pick-up Prozeß setzt sich aus drei GaußKurven zusammen. In der Mitte des Spektrums wird erhöhte Intensiät registriert, weil die Wasserstofffragmente aus der Photodissoziation der Kohlenwasserstoffe größtenteils vollständig in der Clusterumgebung abgebremst werden. Das Untergrundspektrum, daß aus der Photodissoziation der freien Moleküle stammt, führt zu einer Struktur mit vier voneinander getrennten Peaks (s. Abschnitt 3.2.1). Die einzelnen Peaks besitzen dabei die Form von Lorentzkurven. Die in dieser Art geglätteten Untergrundspektren werden von der eigentlichen Messung zum Gewinn des reinen Nutzsignals abgezogen.

\subsection{Transformation der Flugzeitspektren in Energieverteilungen}

Zuerst soll in diesem Abschnitt die Transformation eines Flugzeitspektrums in den Energieraum qualitativ diskutiert werden, bevor in den beiden Unterabschnitten auf die dazu benötigten Programmpakete eingegangen wird.

In Abb. 3.3 ist im oberen Teil ein Wasserstofffragment-Flugzeitspektrum aus der Photodissoziation von $(\mathrm{HBr})_{\mathrm{n}}$ Clustern $(\langle n\rangle=10)$ zu sehen. An diesem Spektrum läßt sich die Charakteristik der Photodissoziation eines freien $\mathrm{HBr}$ Moleküls genauso erkennen, wie der Einfluß des kleinen Clusters in Form des Käfigeffekts. In der Photodissoziation eines freien Moleküls wäre nur der orange gefärbte Teil des Spektrums präsent. Dieser steht für Wasserstofffragmente, die nach der Dissoziation die Umgebung ohne Wechselwirkung verlassen. Verursacht durch die Spin-Bahn-Aufspaltung des Bromatoms kommt es zu einer Doppelpeakstruktur. Die Wasserstofffragmente in den höheren Peaks haben aus der Dissoziation 

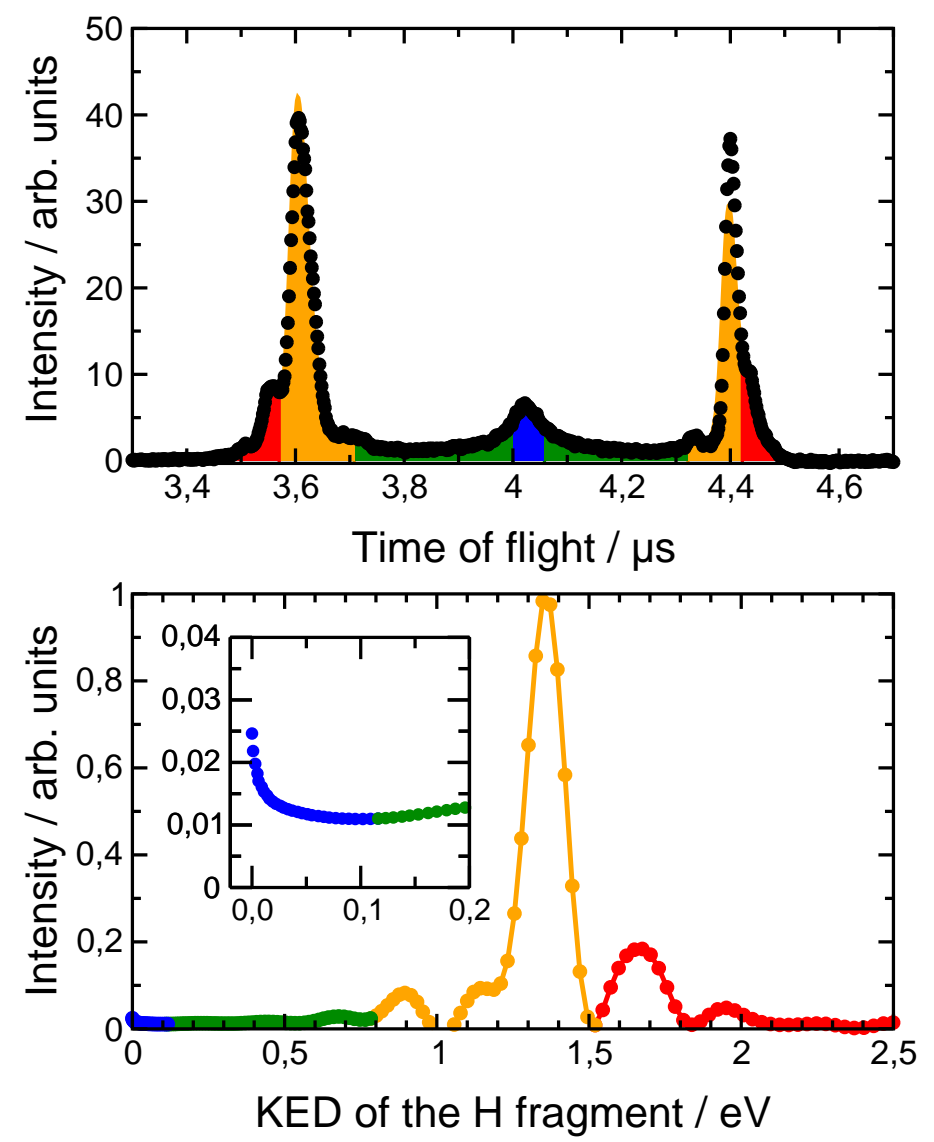

Abbildung 3.3: Transformation eines Wasserstoff-Flugzeitspektrums in den Energieraum. Oberer Teil: Flugzeitspektrum der Wasserstofffragmente aus der Photodissoziation von $(\mathrm{HBr})_{10}$ [Bau99a] symmetrisch zu den Fragmenten ohne Startgeschwindigkeit (blau). Die Symmetrie resultiert aus der Meßmethode im Niedrigfeldmodus (s. Abschnitt 1.3.1). Rechts und links schließen sich im Cluster durch Stöße teilweise abgebremste Fragemente an (grün). Die orangefarbenen Flächen kennzeichnen Wasserstofffragmente, die nach der Dissoziation den Cluster ohne Wechselwirkung verlassen. Die Flugzeiten der Fragmente aus der Photodissoziation von schwingungsangeregte oder rekombinierten $\mathrm{HBr}$ Molekülen sind rot gekennzeichnet. Unterer Teil: Aus dem Flugzeitspektrum transformierte Energieverteilung (KED: kinetic energy distribution) der Wasserstofffragmente. Unterschiede in den Intensitätsverhältnissen der einzelnen Bereiche im Vergleich zum Flugzeitspektrum ergeben sich aus der Faltung mit der Detektionswahrscheinlichkeit im WMTOFMS. 
$\mathrm{Br}$ im Grundzustand als Partnerfragment. In den kleinen Peaks ist das Partnerfragment $\mathrm{Br}^{*}$. Das Intensitätsverhältnis der Peaks zueinander wird durch das Verzweigungsverhältnis bestimmt (s. Abschnitt 1.1).

Im unteren Teil der Abbildung ist die Energieverteilung der Wasserstofffragmente dargestellt. Die eben diskutierten Fragmente sind auch hier orange eingefärbt. Die Zuordnung der Peaks kann an Hand ihrer Intensität erfolgen. Die gemessenen Energiewerte stimmen mit den Literaturwerten (s. Tab. 3.1) überein.

Bedingt durch die Clusterumgebung spielen hier weitere in Abschnitt 1.1.5 diskutierte Einflüsse des Käfigeffekts eine Rolle. Die Fragmente können durch einige Stöße mit dem Clusterkäfig abgebremst werden, was sich in einem Enegieverlust bemerkbar macht (grün), des weiteren können sie auch vollständig abgebremst werden (blauer Peak in der Mitte). Auch diese Teile des Flugzeitspektrums sind in der Energieverteilung wiederzuerkennen, wobei sich gerade bei den Fragmenten ohne kinetische Anfangsenergie die Energieabhängigkeit der Detektionswahrscheinlichkeit bemerkbar macht. Die Detektionswahrscheinlichkeit von eins im Flugzeitraum wird hier gegenüber den anderen Intensitäten im Energieraum korrigiert. Ein leichter Intensitätsanstieg ist in dem vergrößerten Ausschnitt der Energieverteilung zu erkennen. Der rot markierte Bereich ganz außen stammt von Wasserstofffragmenten, die eine höhere Energie besitzen, als sie aus der Dissoziation von $\mathrm{HBr}$ im Grundzustand herrühren könnte. Hierbei handelt es sich um die Photodissoziation von schwingungsangeregten $\mathrm{HBr}$ Molekülen. Die zusätzliche Energie der Schwingungsanregung wird in der Dissoziation in kinetische Energie des Wasserstofffragments umgewandelt. Die Schwingungsanregung basiert auf Stößen oder Rekombinationen.

\subsubsection{Monte-Carlo Simulation der Trajektorien im WM-TOFMS}

Dieses Programm berechnet Trajektorien $T_{i}$ einzelner lonen im Flugzeitmassenspektrometer. Die Kenntnis einzelner lonentrajektorien führt über die Summation dieser Trajektorien unter Berücksichtigung der statischen und dynamischen Störungen zu Flugzeitspektren mit einem definierten Startparametersatz bestehend aus Energie $E_{i}$ und Anisotropiefaktor $\beta_{i}$.

Die Paketstruktur wird in Abbildung 3.4 gezeigt und soll hier erläutert werden.

Der Ablauf beginnt mit dem Start von zwei Programmen zur Erzeugung von Startwerten für die Winkelverteilung $I(\Theta)$ der Fragmente aus dem Anisotropiefaktor $\beta$ sowie der Geschwindigkeitsverteilung $I(v)$ des Ausgangsmoleküls aus dem speed ratio $\mathrm{S}$ des Mo- 
lekularstrahls. Diese Startwerte werden mit einer Gewichtung der Wahrscheinlichkeit in Bibliotheken abgespeichert. Die in den Parameterdateien abgelegten Informationen über benutzte Dissoziationswellenlänge und zu dissoziierendes Molekül werden daraufhin zur Berechnung der kinetischen Energie des Fragments herangezogen und ergänzen die Information über die Winkelverteilung und überlagerte Molekularstrahlgeschwindigkeit. Zum Start der eigentlichen Trajektorienberechnung wird über ein Monte-Carlo-Verfahren ein Startort der Trajektorie im festgelegten Ionisationsvolumen gewählt und die Trajektorie gestartet.

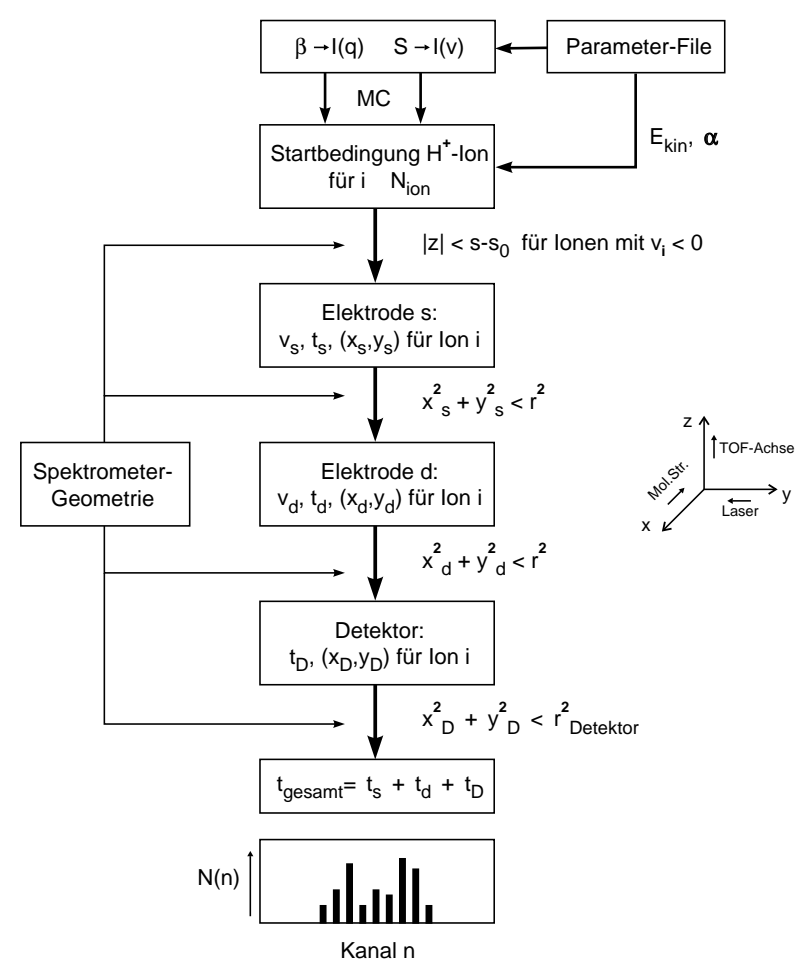

Abbildung 3.4: Ablaufplan zum Programmpaket der Trajektoriensimulation.

Die Bewegung des lons entlang der Detektorachse wird jeweils beim Erreichen einer Blende unterbrochen und auf die Abbruchbedingung, nämlich den Durchtritt durch die Blendenöffnung, hin kontrolliert. Erreicht das lon den Detektor, wird es als nachgewiesen betrachtet und mit seiner Ankunftszeit in einem Zeitfeld von 4096 Feldern der Auflösung 2 ns abgespeichert. In diese Datei werden auch die Ergebnisse der weiteren Ionentrajektorien, deren Gesamtzahl in der Regel einige 10.000 beträgt, abgelegt. 


\begin{tabular}{|c|c|c|c|}
\hline & $\mathrm{HCl}$ & $\mathrm{HBr}$ & $\mathrm{HI}$ \\
\hline Dissoziationsenergie [eV] & $4.432^{\mathrm{a}}$ & $3.745^{b}$ & $3.194^{c}$ \\
\hline Spin-Bahn-Aufspaltung [eV] & $0.109^{a}$ & $0.475^{d}$ & $0.943^{e}$ \\
\hline \multicolumn{4}{|l|}{ Anisotropiefaktor $\beta$} \\
\hline Grundzustand $X$ & $-1.00_{193 \mathrm{~nm}}^{f}$ & $-0.96_{243 n m}^{g}$ & $-1.00_{243 \mathrm{~nm}}^{e}$ \\
\hline angeregter Zustand $X^{*}$ & $-0.94_{193 \mathrm{~nm}}^{f}$ & $1.96_{243 \mathrm{~nm}}^{g}$ & $2.00_{243 \mathrm{~nm}}^{e}$ \\
\hline Verzweigungsverhältnis $R$ & $0.69_{193 \mathrm{~nm}}^{f}$ & $0.2_{243 \mathrm{~nm}}^{g}$ & $0.93_{243 \mathrm{~nm}}^{e}$ \\
\hline \multicolumn{4}{|l|}{ kin. Energie des $\mathrm{H}$-Fragments [eV] } \\
\hline Grundzustand $X$ & $1.998 \mathrm{eV}_{193 \mathrm{~nm}}$ & $1.348_{243} \mathrm{~nm}$ & $1.901_{243 \mathrm{~nm}}$ \\
\hline angeregter Zustand $X^{*}$ & $1.889 \mathrm{eV}_{193 \mathrm{~nm}}$ & $0.891_{243 \mathrm{~nm}}$ & $0.958_{243 \mathrm{~nm}}$ \\
\hline Dipolmoment $\mu[\mathrm{D}]^{\mathrm{h}}$ & 1.109 & 0.83 & 0.43 \\
\hline Polarisierbarkeit $\alpha\left[\AA^{3}\right]^{\mathrm{h}}$ & 2.7 & 3.6 & 5.4 \\
\hline lonisierungsenergie $[\mathrm{eV}]^{\mathrm{h}}$ & 12.75 & 11.66 & 10.39 \\
\hline Bindungslänge $r_{e}[\AA]^{\mathrm{h}}$ & 1.27 & 1.41 & 1.61 \\
\hline Isotope des Halogens ${ }^{h}$ & ${ }^{35} \mathrm{Cl},{ }^{37} \mathrm{Cl}$ & ${ }^{79} \mathrm{Br},{ }^{81} \mathrm{Br}$ & 127 \\
\hline Isotopenhäufigkeit [\%] & $75.77,24.23$ & $50.69,49.31$ & 100 \\
\hline Masse des Halogens $[u]$ & 35.453 & 79.904 & 126.904 \\
\hline Siedepunkt $\left[{ }^{\circ} \mathrm{C}\right]{ }^{\mathrm{h}}$ & -85.05 & -66.73 & -35.36 \\
\hline Schmelzpunkt $\left[{ }^{\circ} \mathrm{C}\right]^{\mathrm{h}}$ & -114.02 & -86.82 & -50.80 \\
\hline
\end{tabular}

Tabelle 3.1: Daten zur Photodissoziation der Wasserstoffhalogenide: a [Liu99], b [Reg99b], ${ }^{\mathrm{c}}$ [Cox91], ${ }^{\mathrm{d}}$ [Moo52], ${ }^{\mathrm{e}}$ [Roy02], ${ }^{\mathrm{f}}$ [Zha97], ${ }^{\mathrm{g}}$ [Bau99b], ${ }^{\mathrm{h}}$ [Lid96]

In das Simulationsprogramm finden als weitere Parameter die Laserpolarisation, die Winkelverteilung und die Geschwindigkeitsverteilung des Strahls Eingang. Hier sind zuerst die Moleküldaten zu nennen, die in Tab. 3.1 aufgeführt sind. Die Dissoziationsenergie, SpinBahn-Aufspaltung, die Massen und Isotopenverteilung werden benötigt, um die kinetische Anfangsenergie des Wasserstoffatoms zu berechnen. Für diese Berechnung wird im weiteren die Photonenenergie des Dissoziationslasers benötigt. Schwankungen in den Feldstärken des Abzugs- und Beschleunigungsfeld sowie des Dissoziationsorts werden mit statistischen Methoden berücksichtigt [Bau99a].

In den Abb. 3.5 und 3.6 sind Tests des Simulationsprogramms zur Photodissoziation von $\mathrm{HBr}$ und $\mathrm{HCl}$ in unserem WMTOFMS mit den experimentellen Parametern durchgeführt 


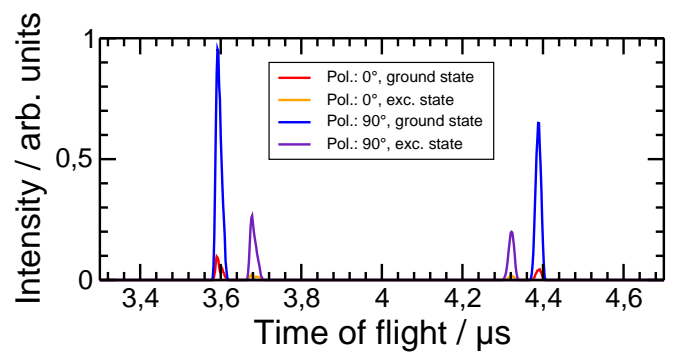

Abbildung 3.5: Monoenergetisches Flugzeitspektrum für die Photodissoziation von $\mathrm{HBr}$ ermittelt mit dem MC-Trajektorien-Simulationsprogramm

worden. Für $\mathrm{HBr}$ (Abb. 3.5) erkennt man in der Intensitätsverteilung die Notwendigkeit des Simulationsprogramms. In der Simulation ist die Winkelverteilung als Anfangsparameter berücksichtigt worden. Daraus ergibt sich damit auch das Verzweigungsverhältnis, was hier graphisch abgelesen 0.27 ergibt. Der Literaturwert, den man auch als Ergebnis aus der Simulation erhält ist jedoch $R=0.2$. Die Abweichung ist auf die Detektionswahrscheinlichkeit zurückzuführen.

Aufgrund der oben beschriebenen Parameter, die Eingang in die Simulation finden, besitzen die Peaks in den berechneten Flugzeitspektren in Parallelität zu den Experimenten eine endliche Breite. Dies führt in der Berechnung des Flugzeitspektrums für $\mathrm{HCl}$ (Abb. 3.6) dazu, daß die einzelnen Peaks aufgrund der geringen Spin-Bahn-Aufspaltung nicht mehr aufgelöst werden. Die Trennung der Peaks ist auch in den Experimenten nicht möglich (s. Abschnitt 4.3.3).

Die Photodissoziation von $\mathrm{HI}$ erlaubt einen aussagekräftigen Test für das Experiment und das Simulationsprogramm. $\mathrm{HI}$ ist das in einer Vielzahl von Experimenten und Rechnungen am genauesten bestimmte Photodissoziatons-System [Roy02]. In Abb. 3.7 ist das experimentelle Flugzeitspektrum aus der Photodissoziation von $\mathrm{HI}$ bei einer Dissoziationswellenlänge von $243 \mathrm{~nm}$ dargestellt (graue Kreise). Die schwarze Kurve ist das Ergebnis aus dem Simulationsprogramm, in dem alle bekannten Parameter unseres Experimentes und die Literaturwerte für die Dissoziationsenergie, Spin-Bahn-Aufspaltung, Anisotropie $(\beta)$ und das Verzweigungsverhältnis $(R)$ berücksichtigt wurden. Die sehr gute Übereinstimmung zeigt die Zuverlässigkeit unserer Meßmethode, mit der sich in Kombination mit dem Simulationsprogramm quantitativ gute Ergebnisse zur Energetik und Winkelverteilung von Photofragmenten erzielen lassen. 


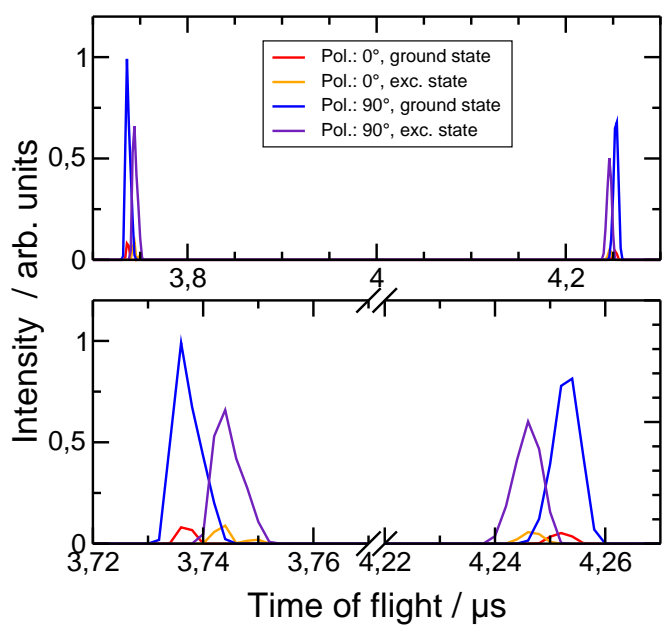

Abbildung 3.6: Monoenergetisches Flugzeitspektrum für die Photodissoziation von $\mathrm{HCl}$ ermittelt mit dem MC-Trajektorien-Simulationsprogramm. Der obere Teil zeigt das Flugzeitspektrum und illustriert die Folgen der energetisch geringen Spin-BahnAufspaltung von $\mathrm{HCl}$, die sich darin äußert, daß die Peaks nicht aufgelöst sind. Der untere Teil stellt die Intensitätsverhältnisse auf einer gestreckten, unterbrochenen Zeitachse nochmal deutlich heraus.

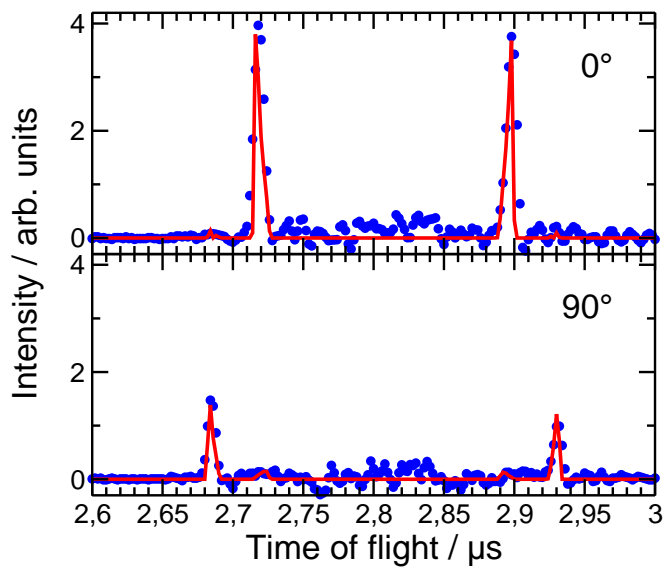

Abbildung 3.7: Photodissoziation von $\mathrm{HI}$ Molekülen bei 243nm, gefittet mit monoenergetischen Flugzeitspektren ermittelt mit dem MC-TrajektorienSimulationsprogramm. Anisotropie: $\beta=-1.00, \beta^{*}=2.00$; Verzweigungsverhältnis: $\mathrm{R}$ $=0.934$; Polarisation: oben $\alpha=0^{\circ}$, unten $\alpha=90^{\circ}$ 


\subsubsection{Transformation poly-energetischer Flugzeitspektren}

Den wichtigsten Teil dieser Arbeit bilden die Energieverteilungen der Wasserstofffragmente aus der Photodissoziation von Halogenwasserstoff-Molekülen in Clusterumgebungen. Die Wechselwirkungen mit dem Käfig bremsen dabei die Fragmente in unterschiedlichem Maße ab. Dies führt zu poly-energetischen Flugzeitspektren.

Innerhalb der Arbeitsgruppe wurde dazu ein Programmpaket entwickelt, daß eine solche Energieverteilung ermittelt. In der Konzeption dieses Programmpakets wurde dazu das gemessene Flugzeitspektrum $F$ als Summation von mono-energetischen Flugzeitspektren $f$ aufgefaßt, die aus dem oben beschriebenen MC Trajektoriensimulationsprogramm stammen

$$
F=\sum_{i} k_{i} \cdot f_{i}
$$

Es zeigte sich allerdings während der Programmentwicklung, daß die alleinige Benutzung von mono-energetischen Spektren zur Anpassung an das gemessene Spektrum zu Auflösungsproblemen führt, da die mono-energetischen Spektren per Definition einer deltaförmigen Apparaturauflösung entsprechen. Um die Energieauflösung der simulierten Spektren dem Massenspektrometer anzupassen, wurde deshalb eine der Detektorauflösung entsprechende Abdeckung des Energieintervalls mit Gaußkurven der Halbwertsbreite $b_{g a u}$ vorgenommen. Diese Gaußkurven vermeiden eine Überinterpretation des im gemessenen Spektrum vorhandenen Rauschens durch die Simulation.

Entsprechend den Energiestützpunkten $E_{n}$ im Intervall wird für jede der Gaußverteilungen an der Stelle $g_{j}$ ein neues Flugzeitspektrum $G\left(g_{j}\right)$ aus den gewichteten Spektren $f$ gebildet

$$
G\left(g_{j}\right)=\sum_{n} e^{-\frac{\left(E_{n}-G_{j}\right)^{2}}{b_{g a u}}} \cdot f\left(E_{n}\right)
$$

und ein Gleichungssystem aufgestellt

$$
F=\sum_{j} c_{j} \cdot G_{j}
$$

Die genaue numerische Lösung dieses Gleichungssystms mittels Inversion der regulären Koeffizientenmatrix (Gauß-Jordan-Eliminationsverfahren) ist in [Bau99a] erläutert. Daraus erhält man die transformierte Energieverteilung und einen Fit des Flugzeitspektrums, 
dessen Abweichung vom gemessenen Spektrum eine Aussage über die Zuverlässigkeit der Transformation erlaubt. 


\section{Experimentelle Ergebnisse}

Die experimentellen Ergebnisse der Photodissoziationsexperimente von $\mathrm{HX}(\mathrm{X}=\mathrm{I}, \mathrm{Br}, \mathrm{Cl})$ in Edelgasclusterumgebungen werden in diesem Kapitel vorgestellt.

Für die Photodissoziation von HI Molekülen auf Ar Clustern wird die Bevölkerung der Produktkanäle in Abhängigkeit von der Polarisation des Dissoziationslasers betrachtet.

Ein größerer Abschnitt beschäftigt sich mit der Photodissoziation von $\mathrm{HBr}$. Dabei wird die Energieverteilung der Wasserstofffragmente in Abhängigkeit von der Clusterquellentemperatur (Expansion von $\mathrm{Ar}$ ) untersucht. Im weiteren wird der Käfigeffekt für $\mathrm{HBr}$ Moleküle in Ar Clustern in Abhängigkeit von der Ar Clustergröße studiert. Bei der Photodissoziation von $\mathrm{HBr}$ auf großen Ne Clustern stellte sich heraus, daß die Messungen Aufschluß über den Aggregatzustand und einen eventuellen Phasenübergang des Clusters in Abhängigkeit von seiner Größe liefern können.

Die Photodissoziation von $\mathrm{HCl}$ in Edelgasclusterumgebungen ist Gegenstand zahlreicher theoretischer Betrachtungen [Kry97, Niv97, Bau00, Niv99, Žd'á99, Žd'á00, McC93, Gar94, Pic01]. Die ersten Experimente auf diesem Gebiet werden in dieser Arbeit durchgeführt. Es werden die Energieverteilungen der Wasserstofffragmente in Abhängigkeit vom Wirtscluster ( $\mathrm{Ne}, \mathrm{Ar}, \mathrm{Kr}, \mathrm{Xe}$ ) sowie die Größenabhängigkeiten exemplarisch an $\mathrm{Ne}$ und Ar Clustern vorgestellt. Bei diesen Experimenten wird der Cluster mittels Pick-up Technik präpariert, was zu einer Einlagerung der Moleküle in die Clusteroberfläche führt. Weiter liegen Ergebnisse zur Photodissoziation von $(\mathrm{HCl})_{\times}$Clustern und $(\mathrm{HCl})_{\times}-\mathrm{Ar}_{\mathrm{n}}$ Mischclustern aus der Koexpansion $\mathrm{HCl}$ in $\mathrm{Ar}$ vor. Die Koexpasion führt zu einer Einbettung der $(\mathrm{HCl})_{\times}$Komplexe in den großen $\mathrm{Ar}_{n}$ Cluster. Eine Untersuchung in Abhängigkeit von der Polarisation des Dissoziationslasers wird ebenfalls vorgenommen.

Die Ergebnisse im letzten Abschnitt dieses Kapitels resultieren aus asymmetrischen Flugzeitspektren der Photodissoziation von $\mathrm{HI}, \mathrm{HBr}$ und $\mathrm{HCl}$ auf $\mathrm{Xe}$ Clustern. Sie werden der Bildung, Orientierung und Dissoziation von $\mathrm{HXel}, \mathrm{HXeBr}$ und $\mathrm{HXeCl}$ zugeschrieben (vgl. Abschnitt 1.4). 


\subsection{Photodissoziation von HI Molekülen auf Ar Clustern}

Es gibt bereits Messungen aus unserer Gruppe zur Photodissoziation von $\mathrm{HI}$ adsorbiert auf Edelgasclustern [Bau01a, Bau99a]. Für die hier beschriebenen Messungen liegt das Augenmerk auf der genauen Position der HI Moleküle in der Umgebung der Ar Clusteroberfläche bei Präparation mittels Pick-up. Im Mittelpunkt der Untersuchungen stehen die an der Photodissoziation von $\mathrm{HI}$ beteiligten Potentialflächen und Ausgangskanäle. Diese werden mit unterschiedlicher Polarisation des Dissoziationslasers untersucht (s. Abschnitt 5.2).

Experimentelle Parameter zu den Messungen, was die Strahleigenschaften und die Einstellungen des Dissoziationslasers sowie des Flugzeitmassenspektrometers anbetrifft, sind in Tab. 4.1 aufgelistet.

Es wird ein Ar Clusterstrahl mit einer mittleren Clustergröße von $\langle n\rangle=139$ erzeugt. Die HI Moleküle gelangen im zweiten Präparationsschritt mittels Pick-up auf die Ar Cluster. Dabei ist der Pick-up-Zellendruck so eingestellt, daß im Mittel nur ein HI Molekül an einen Ar Cluster angelagert wird [Bau00]. Weitere Details zur Strahlerzeugung und Pick-up Präparation sind in [Bau99a] nachzulesen. Für diese Messungen kommt das in Abschnitt 2.3.1 beschriebene WMTOFMS mit der kürzeren Driftstrecke zum Einsatz. Die aufgenommenen Flugzeitspektren sind eine Mittlung über 10000 Einzelschußspektren.

Die Messungen sind in Abb. 4.1 aufgeführt. Die gemessenen Flugzeitspektren der Wasserstofffragmente befinden sich auf der linken Seite des Bildes. Die obere Reihe zeigt die Ergebnisse für eine Laserpolarisation von $0^{\circ}$ zur Detektorachse, die untere für $90^{\circ}$. Die gefüllten Kreise $(\bullet)$ kennzeichnen die Meßpunkte, die durchgezogene Linie stammt aus der Simulation der TOF-Spektren und dient als Grundlage zur Transformation in den Energieraum.

Die Energieverteilungen der Wasserstofffragmente sind im linken Teil des Bildes zu sehen. Sie zeichnen sich durch hohe Intensität bei $E_{k i n}=0 \mathrm{eV}$ aus, was dem Anteil völlig abgebremster Wasserstofffragmente entspricht (perfect caging). Ein weiteres Merkmal ist die Doppelpeakstruktur bei 1.9 und $1.0 \mathrm{eV}$. Diesen Intensitäten entsprechen Wasserstofffragmente, die ohne Energieverlust durch Stöße mit dem Käfig den Cluster verlassen. Die Trennung in die beiden Peaks ist auf die Spin-Bahn Aufspaltung bei der Photodissoziation von Halogenwasserstoffen zurückzuführen (Grundzustand I: $1.9 \mathrm{eV}$, angeregter Zustand I*: $1.0 \mathrm{eV}$ ). Ihre Energetik entspricht der von Wasserstoffatomen aus der Photodissoziation von freien HI Molekülen. Im Gegensatz zur skalaren Größe der kinetischen 
- Düsendurchmesser der konischen Düse $d^{*} \quad 60 \mu \mathrm{m}$

- Länge der konischen Düse $\quad l_{d} 2 \mathrm{~mm}$

- Öffnungswinkel des Düsenkonus $\quad \alpha_{D} \quad 30^{\circ}$

- äquivalenter Düsendurchmesser $\quad d_{a q} \quad 164.8 \mu \mathrm{m}$

- stoffspezifische Konstante für Ar $\quad K_{c h} \quad 1646 \frac{\mathrm{K}^{2.2875} \mu \mathrm{m}^{0.85}}{\mathrm{mbar}}$

- Expansionsdruck $\quad p \quad 3.8 \mathrm{bar}$

- Düsentemperatur $\quad T_{D} \quad-57^{\circ} \mathrm{C}$

- mittlere Clustergröße $\quad\langle n\rangle \quad 139$

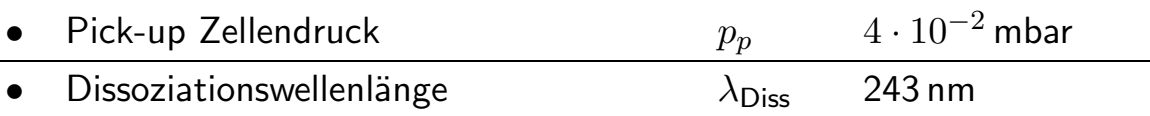

- Laserenergie im TOF-Zentrum $\quad E_{L} \quad 1.54 \mathrm{~mJ}$

- Winkel zwischen Laserpolarisierung und Detektorachse $\quad \alpha \quad 0^{\circ}$ oder $90^{\circ}$

- WMTOFMS mit kurzer Driftstrecke D $169.9 \mathrm{~mm}$

- Abzugsspannung $U_{s} \quad 4.04 \mathrm{~V}$

- Beschleunigungsspannung $\quad U_{d} \quad 12.29 \mathrm{~V}$

- MCP-Spannung $U_{M C P} \quad 1.9 \mathrm{kV}$

- Anisotropiefaktor $\beta$ für freien Ausgang

$\begin{array}{lll}\text { Grundzustand } X & \beta & 0.0 \pm 0.1 \\ \text { angeregter Zustand } X^{*} & \beta^{*} & 0.3 \pm 0.2 \\ \text { weigungsverhältnis } & R & 0.85 \pm 0.1\end{array}$

$\begin{array}{lll}\text { - Verzweigungsverhältnis } & R & 0.85 \pm 0.1\end{array}$

- Verhältnis der Intensität zwischen caged und direct exit

$\begin{array}{lll}\text { Grundzustand } X, 0^{\circ} & R_{g s}^{0} & 4.4 \\ \text { angeregter Zustand } X^{*}, 0^{\circ} & R_{e s}^{0} & 2.0 \\ \text { Grundzustand } X, 90^{\circ} & R_{g s}^{90} & 3.0 \\ \text { angeregter Zustand } X^{*}, 90^{\circ} & R_{e s}^{90} & 3.7\end{array}$

Tabelle 4.1: Experimentelle Parameter zur Photodissoziation von HI Molekülen adsorbiert auf Ar Clustern: Im oberen Teil sind die Strahldaten aufgeführt, anschließend die Laserdaten, im folgenden die Einstellungen des WMTOFMS und abschließend Ergebnisse aus der Photodissoziation 
Energie der Fragmente unterscheidet sich deren Winkelverteilung (vektoriell) deutlich von der des freien Moleküls (s. Abb. 4.2).
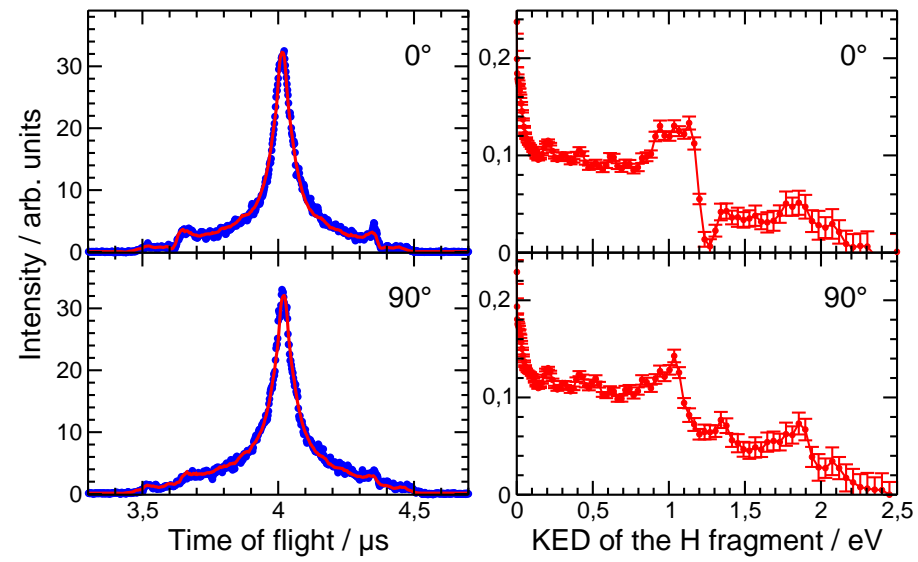

Abbildung 4.1: Photodissoziation von $\mathrm{HI}$ Molekülen auf $\operatorname{Ar}_{\mathrm{n}}$ Clustern $(\langle\mathrm{n}\rangle=139)$. Der Dissoziationslaser ist parallel $\left(0^{\circ}\right)$ oder senkrecht $\left(90^{\circ}\right)$ zur Detektorachse polarisiert. Der linke Teil zeigt die Flugzeitspektren, gemessen $(\bullet)$ und simuliert $(-)$. Es ist ein großer Anteil H Fragmente erkennbar, die keine kinetische Energie besitzen (caged). Ein Teil der Wasserstofffragmente kann den Cluster ungestört verlassen. Der rechte Teil der Abb. zeigt die zugehörigen Energieverteilungen.

Der mittlere Teil der Energieverteilung zwischen 0.1 und $0.8 \mathrm{eV}$ charakterisiert Wasserstofffragmente, die teilweise abgebremst sind. Ihr Energieverlust resultiert aus Wechselwirkungen mit den Käfigatomen. Die Anzahl der Atome, mit denen Wechselwirkung stattfindet ist entscheidend für den Grad an Energieverlust. Nach einigen Wechselwirkungen gelingt es ihnen den Cluster zu verlassen (delayed exit). Die Struktur, die in diesem Bereich in den Energieverteilungen zu erkennen ist, stammt nicht von einer Feinstruktur oder ist ein Indiz für die Anzahl der Wechselwirkungen, sondern sie resultiert aus der Überbestimmung des linearen Gleichungssystems zur Transformation der Flugzeitspektren in Energieverteilungen für diesen niederenergetischen Bereich (s. Abschnitt 3.2.2). Dies führt in der Simulation zu Pseudo-Strukturen. Auffällig in diesen Spektren ist der besonders hohe Anteil nur teilweise abgebremster Wasserstofffragmente, der in der späteren Diskussion 5.1 um die Position des HI Moleküls in der Ar Clusterumgebung eine Rolle spielt.

Die Messungen zeigen, daß die Wahl der Laserpolarisation auf die Intensitätsverteilung der völlig und teilweise abgebremsten Fragmente keinen Einfluß hat. Im Bereich des freien Austritts ist eine Abhängigkeit der Winkelverteilung von der Laserpolarisation zu 
beobachten. Für eine eingehende Analyse bietet sich eine Separation der Flugzeitspektren in die Anteile von teilweise und völlig abgebremsten Fragmenten (perfect caging + delayed exit) auf der einen Seite und den Anteil der ungehindert austretenden Fragmente (free exit) auf der anderen Seite an. Dafür wird von der Energieverteilung der Bereich des Grundzustandes $(1.9 \mathrm{eV})$ und des angeregten Zustandes $(1.0 \mathrm{eV})$ separiert. Im Bereich des angeregten Zustandes ist noch eine zusätzliche Korrektur anzubringen. Hier liegt eine Überlagerung von frei austretenden Wasserstofffragmenten (Partnerfragment ${ }^{*}$ ) und teilweise abgebremsten Wasserstofffragmenten (Partnerfragment I) vor. Letztere müssen von der Gesamtintensität abgezogen werden. In Abb. 4.2 sind zum Vergleich Flugzeitspektren aus der Photodissoziation reiner HI Moleküle (näheres s. Abschnitt 3.2.1) und die separierten Flugzeitspektren für den freien Austritt aus der Photodissoziation von HI Molekülen adsorbiert auf Ar Clustern dargestellt.

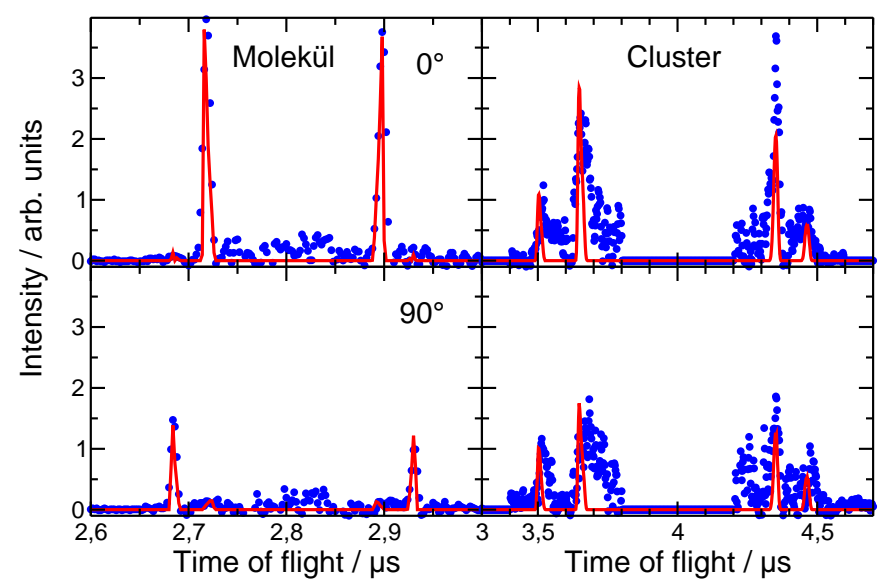

\begin{abstract}
Abbildung 4.2: Photodissoziation von $\mathrm{HI}$ : Vergleich Molekül - Ar Clusterumgebung: Im linken Teil der Abbildung sind Flugzeitspektren aus der Photodissoziation von HI Molekülen (genaue Erläuterung s. Abb. 3.7) mit unterschiedlicher Laserpolarisation zu sehen (Messung $(\bullet)$, Simulation (-), WMTOFMS mit langer Driftstrecke). Die Simulationsparameter sind $\beta=-1.00, \beta^{*}=2.00$ und $R=0.934$ (Werte aus [Roy02]). Im rechten Teil ist aus der Messung in Abb. 4.1 der Teil der Wasserstofffragmente mit direktem Ausgang separiert dargestellt. Für den Grundzustand (Intensitäten außen) ermittelt man einen Anisotropiefaktor $\beta=0.0 \pm 0.1$, für den angeregten Zustand (Intensitäten innen) $\beta^{*}=0.3 \pm 0.2$. Das Verzweigungsverhältnis beträgt $R=0.85 \pm 0.1$.
\end{abstract}


Die starke Anisotropie aus der Photodissoziation von reinem $\mathrm{HI}\left(\beta=-1.00, \beta^{*}=\right.$ 2.00) geht in der Clusterumgebung fast vollständig verloren. Der Grundzustand liefert eine völlig isotrope Verteilung $(\beta=0.0 \pm 0.1)$ und der angeregte Zustand eine nahezu isotrope Verteilung, die einen leicht erhöhten Anteil paralleler Übergänge aufweist $\left(\beta^{*}=\right.$ $0.3 \pm 0.2$ ). Die Veränderung im Verzweigungsverhältnis ist gering (Molekül: $R=0.934$, Cluster: $R=0.85 \pm 0.1$ ). Wie diese Veränderungen mit der Interpretation der Anregung, den Kopplungen und Produktkanälen verknüpft sind, wird in Abschnitt 5.2 diskutiert. Dort findet auch ein Vergleich mit theoretischen Berechungen aus der Gruppe Jungwirth statt.

\subsection{Photodissoziation von $\mathrm{HBr}$ Molekülen in unterschiedlichen Clusterumgebungen}

$\mathrm{HBr}$ ist in unserer Gruppe als Modellsystem zur Photodissoziation benutzt worden, so $\mathrm{da} ß$ es schon zahlreiche Messungen zur Photodissoziation von $\mathrm{HBr}$ Molekülen, $(\mathrm{HBr})_{\mathrm{n}}$ Clustern [Bau95, Fri97, Nah98, Bau99b], HBr Molekülen in Edelgasclusterumgebungen [Bau00, Sla00, Bau01a] und $\mathrm{HBr}_{x}-\mathrm{Ar}_{n}$ Mischclustern [Bau01c] gibt. Trotzdem sind noch einige Fragestellungen offengeblieben. Zum einen, wie verändert sich die Adsorption (Position, Eindringtiefe, Ausrichtung) von $\mathrm{HBr}$ auf Ar Clustern, wenn diese eine unterschiedliche Temperatur (innere Energie) besitzen? Die andere Frage, die noch nicht geklärt ist: Wie viele Ar Clusterschalen benötigt man, um in der Photodissoziation von $\mathrm{HBr}$ im Zentrum des Clusters perfect caging zu erreichen? Im Vergleich dazu werden Ergebnisse zur Photodissoziation von $\mathrm{HBr}$ in $\mathrm{Ne}$ Clustern dargestellt, wo die Frage nach der Position des $\mathrm{HBr}$ Moleküls in der Clusterumgebung mit der Frage nach der Beschaffenheit des Clusters verknüpft ist. Sind Ne Cluster im untersuchten Größenbereich fest oder flüssig?

\subsubsection{Temperaturabhängigkeit der Energieverteilungen aus der Photodissoziation von $\mathrm{HBr}$ Molekülen auf Ar Clustern konstanter Größe}

Die Temperatur der Ar Cluster aus der Düsenstrahlexpansion läßt sich nur indirekt durch Variation der Düsentemperatur beeinflussen. Dabei wird die Clustergröße durch gleichzeitige Anpassung des Expansionsdrucks konstant gehalten (s. GI. 1.24). Genaue Korrelationen zwischen Expansionsbedingungen und Clustertemperatur existieren bisher für Edelgascluster nicht. 
Durch die adiabatische Expansion in der Düse findet eine Abkühlung statt, die mit der Aufheizung durch die Kondensation (Clusterbildung) konkurriert. Der heiße Cluster kann durch Stoßabregung (Monomer-Cluster- oder Cluster-Cluster-Stöße) oder Verdampfungskühlung (evaporative cooling) wieder Wärme abgeben.

Mit der Erhöhung der Düsentemperatur wird die Expansion wärmer. Dies überträgt sich energetisch auch auf den Cluster. Dieser kann tatsächlich eine höhere Temperatur besitzen oder auf diese Energieerhöhung mit Vedampfungskühlung reagieren. In diesem Prozeß verringert sich die Temperatur des Clusters und dessen Größe durch das Abdampfen von Monomeren.

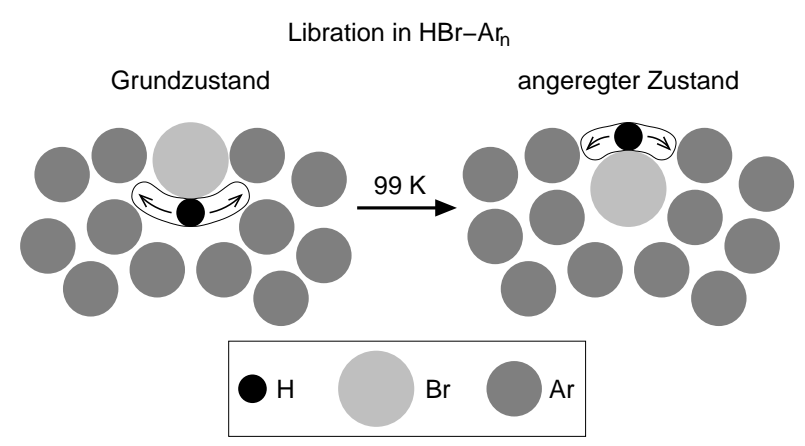

\begin{abstract}
Abbildung 4.3: Libration von $\mathrm{HBr}$ in $\mathrm{Ar}_{\mathrm{n}}$. Bei der Grundzustandskonfiguration zeigt das Wasserstoffatom in den Cluster, in der energetisch um $99 \mathrm{~K}$ höheren Konfiguration (angeregter Zustand) zeigt es nach außen. Die Pendelschwingung ist durch die Fläche gekennzeichnet, in der sich das Wasserstoffatom befinden kann.
\end{abstract}

Ein Unterschied zu den vorhergehenden Betrachtungen reiner Ar Cluster ist in diesen Experimenten durch den Einbau eines $\mathrm{HBr}$ Moleküls in die Ar Clusteroberfläche gegeben. Wenn es nun gelingt, den Ar Cluster zu heizen, stellt sich die Frage, ob diese Energie auf das $\mathrm{HBr}$ Molekül übertragen wird.

Die Gruppe um P. Jungwirth hat Berechnungen zur Orientierung von Halogenwasserstoff-Molekülen auf Edelgasclusteroberflächen durchgeführt [Sla01]. Ob das Wasserstoffatom zum Cluster oder von ihm weg zeigt, wird von den konkurrierenden Prozessen der Induktion und Dispersion bestimmt ${ }^{1}$. Der Komplex $\mathrm{H}-\mathrm{X}-\mathrm{Rg}_{\mathrm{n}}$

\footnotetext{
${ }^{1}$ Induktion: Wechselwirkung zwischen der Ladungsverteilung eines Komplexes und der dadurch in einem zweiten Komplex induzierten Ladungsverteilung. Dispersion: Wechselwirkung zwischen einer fluktuierenden Ladungsverteilung in Komplex eins und der dadurch induzierten fluktuierenden Ladung in Komplex zwei. Berechnungen der Induktion und Dispersion sind in Störungsrechnung zweiter Ordnung möglich.
} 
wird gebildet, wenn die Dispersion überwiegt. Das Wasserstoffatom zeigt zum Cluster $\left(\mathrm{X}-\mathrm{H}-\mathrm{Rg}_{\mathrm{n}}\right)$, wenn die Induktion dominiert. Für die Orientierung von $\mathrm{HBr}$ Molekülen auf großen Ar Clustern ist die Induktion maßgebend, so daß der Komplex $\mathrm{Br}-\mathrm{H}-\mathrm{Ar}_{n}$ das energetische Minimum bildet. Das HBr Molekül führt in dieser Konfiguration im Grundzustand wegen der geringen Masse des Wasserstoffatoms Nullpunktsschwingungen der verhinderten Rotation (Libration) aus, die in Abb. 4.3 illustriert ist. Das Wasserstoffatom kann aufgrund der Behinderung durch den Ar Käfig nur Pendelschwingungen ausführen. Es spielt keine Rolle, in welcher Position sich das Wasserstoffatom zum Zeitpunkt einer Photodissoziation befindet, das Wasserstofffragment wird immer mit mehreren Ar Atomen wechselwirken, wodurch es stark abgebremst wird (delayed exit oder perfect caging).

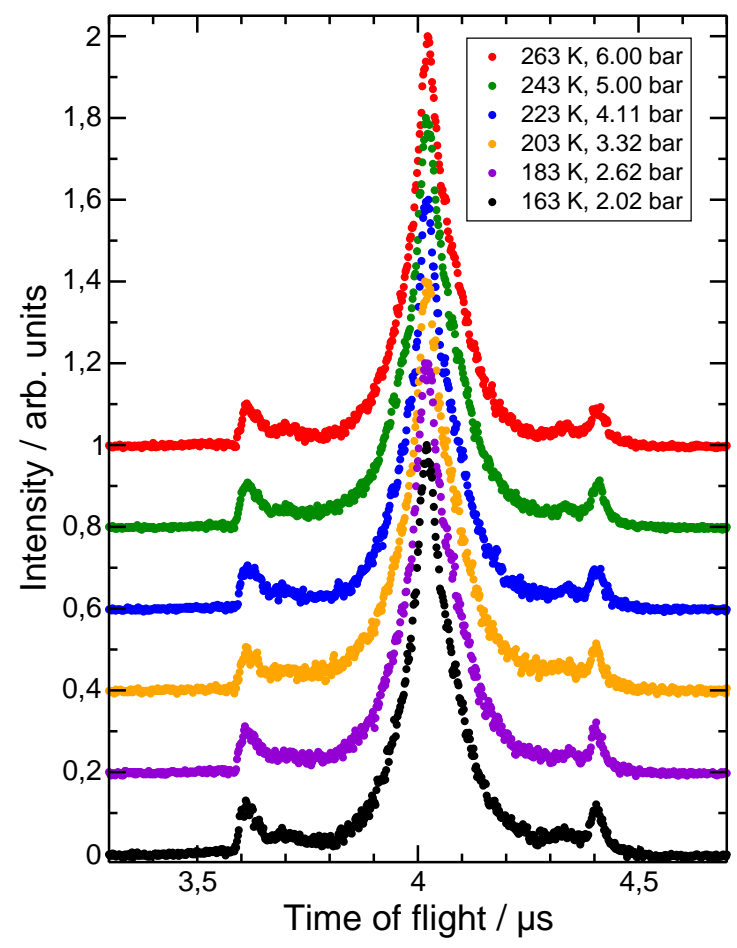

Abbildung 4.4: Flugzeitspektrum der Photodissoziation von einem HBr Molekül auf geheizten $\operatorname{Ar}$ Clustern mit $\langle n\rangle=139$ : Die Größe wird konstant gehalten, indem parallel zu einer Temperaturänderung auch der Druck angepasst wird. Die Variation der Düsentemperatur deckt den Bereich von 163 - $263 \mathrm{~K}$ ab. Dies ist mit einem Druckbereich von $2.02-6.00$ bar korreliert. Es ist nur eine geringfügige Änderung der Spektren zu beobachten (s.Abb.4.5). 
Im ersten angeregten Zustand dieser Pendelbewegung, der energetisch $68.3 \mathrm{~cm}^{-1}$ höher liegt, zeigt das Wasserstoffatom aus dem Cluster heraus und kann diesen ohne Wechselwirkung mit den Ar Atomen verlassen (free exit).

Experimentell soll hier herausgefunden werden, ob eine Erhöhung der Düsentemperatur zu einer thermischen Anregung des Ar Clusters führt, die zu Bildung der Struktur $\mathrm{H}-\mathrm{Br}-$ $\operatorname{Ar}_{\mathrm{n}}$ führt.

Die Experimente zu dieser Thematik haben ähnliche Molekularstrahl- und Laserbedingungen wie für die Photodissoziation von $\mathrm{HI}$ auf $\mathrm{Ar}$ Clustern (Tab. 4.1). Die mittlere $\mathrm{Ar}$ Clustergröße beläuft sich auf $\langle n\rangle=139$, der $\mathrm{HBr}$ Pick-up Druck liegt bei $4 \cdot 10^{-2} \mathrm{mbar}$ und die Polarisation des Dissoziationslasers ist senkrecht zur Detektorachse. Die Düsentemperatur wird zwischen 163 und $263 \mathrm{~K}$ variiert. Die Clustergröße wird über die Anpassung des Drucks (2-6 bar) konstant gehalten. Die Messungen in Abb. 4.4 zeigen jedoch fast keine Temperaturabhängigkeit.

Welchen Einfluß hat eine Veränderung der Temperatur auf die Flugzeitspektren? Den stärksten Einfluß auf die Spektren hätte die Anregung der Librationsmode. Das Wasserstoffatom würde während der Dissoziation nach außen zeigen und würde damit zum gleichen Flugzeitspektrum wie die Photodissoziation eines freien Moleküls führen. Es gäbe keine abgebremsten Wasserstofffragmente.

Wenn der Cluster flüssig wäre, dränge das $\mathrm{HBr}$ Molekül in den Cluster ein, und man erhielte ein Spektrum wie in Abb. 4.6. Dabei liegt im Flugzeitraum bei einer ClustergröBe von $\langle n\rangle=139$ (oberstes linkes Bild in 4.6) ein Intensitätsverhältnis zwischen völlig abgebremsten und ungehindert austretenden Fragmenten von $R_{T O F}=28$ vor. Für diese Messungen bei geheizten Ar Clustern beträgt das Verhältnis je nach Düsentemperatur $7.6<R<10.4$ (s. Abb. 4.5). Dieses Resultat spricht gegen geschmolzene Ar Cluster, wobei das Verhältnis bei Erhöhung der Düsentemperatur leicht ansteigt.

Der dritte oben genannte Effekt, das Abdampfen von Monomeren, würde dahingehend zu einer Änderung des Flugzeitspektrums führen, daß die Gesamtintensität abnähme [Bau00]. Dies ist aus Abb. 4.4 nicht ablesbar, weil die Spektren in ihrer Maximalintensität auf eins normiert sind. Die nicht normierten Spektren zeigen abgesehen von gewöhnlichen Schwankungen in den Messungen keine Temperatur- bzw. Größenabhängigkeit. Wenn man davon ausgeht, daß die Bindungsenergie der Ar Atome im Cluster bei ungefähr $60 \mathrm{meV}$ liegt ${ }^{2}$, was energetisch einer Temperatur von $700 \mathrm{~K}$ entspricht, bedeutet das, daß bei einer Temperaturerhöhung um $100 \mathrm{~K}$ ungefähr 20 Monomere pro Cluster abgedampft werden

\footnotetext{
${ }^{2}$ Bindungsenergie Ar Dimer: $10 \mathrm{meV}$, Ar Atom im Festkörper: $80 \mathrm{meV}$
} 


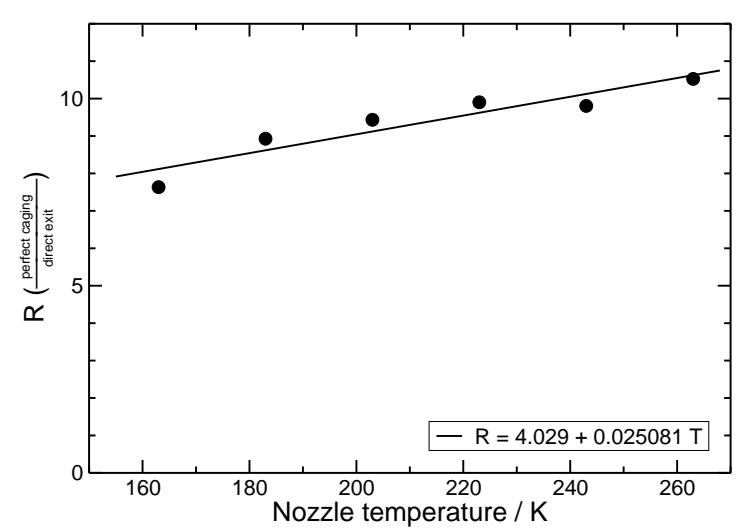

Abbildung 4.5: Käfig-Effekt von $\mathrm{HBr}$ auf Ar Clustern in Abhängigkeit von der Clustertemperatur: Aus den Flugzeitspektren (Abb. 4.4) läßt sich das Verhältnis R für Wasserstofffragmente, die vollkommen im Cluster abgebremst werden (caged), zu denen, die den Cluster ungehindert verlassen (exit), berechnen. Die Abhängigkeit von der Düsentemperatur ist in dieser Abb. zu sehen. Bei höheren Düsentemperaturen nimmt der Anteil der Wasserstofffragmente, die im Cluster vollständig abgebremst werden, zu.

könnten (evaporative cooling). Dies würde aber voraussetzen, daß sich die Erhöhung der Düsentemperatur direkt energetisch in den Cluster übertragen müßte, wovon nicht auszugehen ist. Die Zahl von 20 Monomeren ist damit als Abschätzung nach oben zu verstehen.

Wie oben bereits erwähnt, müßte eine Veränderung im Intensitätsverhältnis der Wasserstofffragmente, die vollständig abgebremst (perfect caging) werden zu denen, die den Cluster unbeeinflußt verlassen (direct exit), eintreten. In Abb. 4.5 ist dieses Verhältnis $R$ in Abhängigkeit von der Düsentemperatur aufgetragen. Man erkennt dort einen Anstieg hin zu höheren Düsentemperaturen. Wie dies mit der Position des $\mathrm{HBr}$ Moleküls in der $\mathrm{Ar}$ Clusteroberfläche korreliert ist, wird intensiv in der Diskussion im Abschnitt 5.1 behandelt.

\subsubsection{Photodissoziation von $\mathrm{HBr}$ Molekülen in Ar Clustern: Käfigeffekt in Abhängigkeit von der Clustergröße}

Im Gegensatz zu den Experimenten in den vorherigen Abschnitten, kommt bei der Erzeugung von $\mathrm{HBr}$ Molekülen in $\mathrm{Ar}$ Clustern eine andere Präparationsmethode zum Einsatz. Die Cluster werden nicht mittels Pick-up Verfahren, sondern per Koexpansion von $\mathrm{HBr}$ in Ar erzeugt (s. Abschnitt 1.2.3). 
Im Hinblick auf den Käfigeffekt stellt sich die Frage: Wie viele Ar Clusterschalen müssen ein $\mathrm{HBr}$ Molekül umgeben, damit bei der Photodissoziation nur noch vollständig abgebremste Wasserstofffragmente auftreten (perfect caging)?
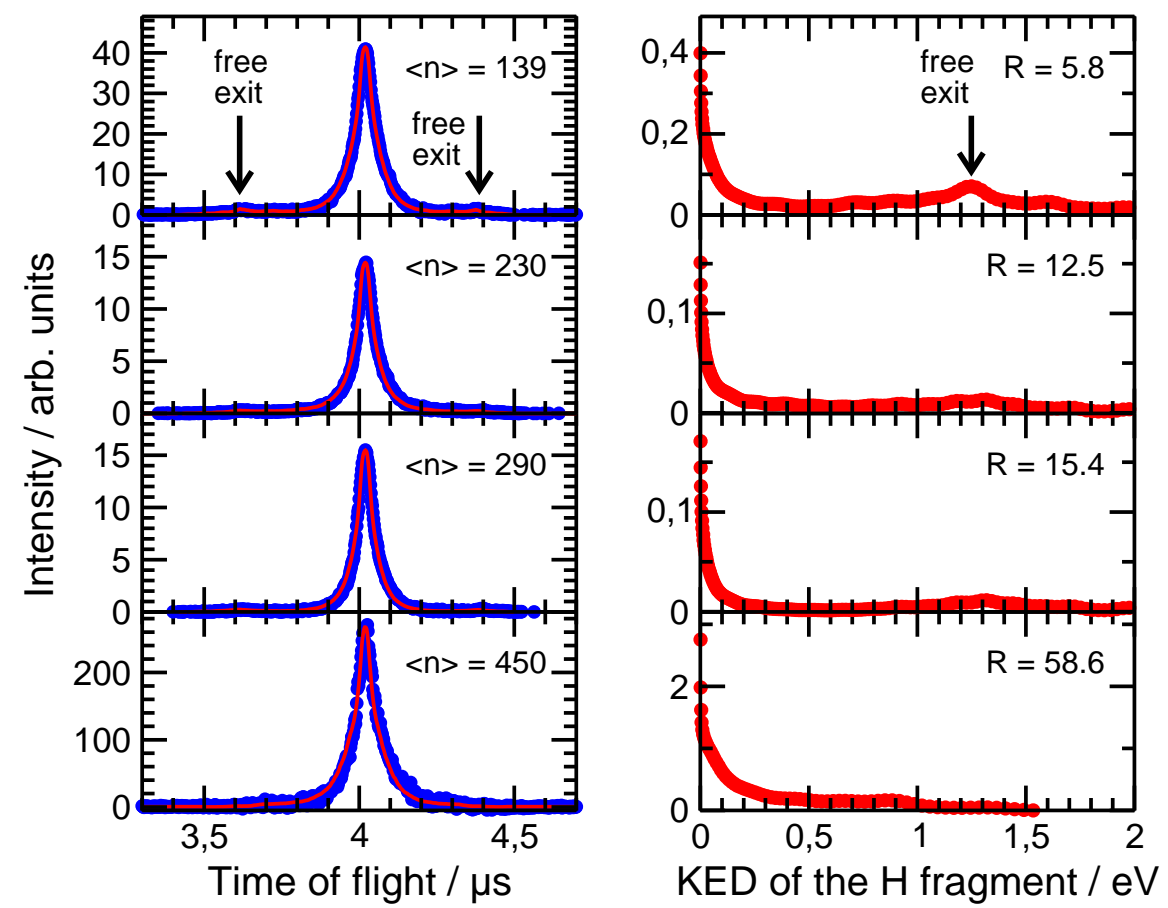

Abbildung 4.6: Photodissoziation von einem $\mathrm{HBr}$ Molekül in großen $\mathrm{Ar}_{\mathrm{n}}$ Clustern (mittlere Clustergröße $\langle n\rangle=139-450)$. Gemessenes $(\bullet)$ und simuliertes $(-)$ Flugzeitspektrum links und Energieverteilung rechts. Der ungestörte Ausgang des Wasserstoff fragments (gekennzeichnet mit free exit) nimmt mit zunehmender Clustergröße ab. R beschreibt das Verhältnis der Intensitäten bei $E_{k i n}=0 \mathrm{eV}$ (caged) zu $E_{k i n}=1.35 \mathrm{eV}=D_{0}-h \nu$ (free exit). Die hohe Intensität ist für $\langle n\rangle=139$ auf eine höhere Laserintensität, für $\langle n\rangle=450$ auf die Mittlung über 100 Spektren mit je 100 Laserpulsen zurückzuführen.

Dafür werden Experimente zur Photodissoziation von $\mathrm{HBr}$ bei einer Dissoziationswellenlänge von $243 \mathrm{~nm}$ in Ar Clustern unterschiedlicher Größe $(139<\langle n\rangle<450)$ durchgeführt. Andere Einflüsse als der des Ar Käfigs auf die Photodissoziation sollen vermieden werden. Dafür ist es insbesondere notwendig, die Cluster so zu präparieren, daß sich nur ein $\mathrm{HBr}$ Molekül im Ar Cluster befindet. Dies läßt sich über das Mischungsverhältnis bei der Expansion steuern. Die hier verwendeten Mischungen liegen zwischen $0.05 \%$ und $0.2 \%$ 
$\mathrm{HBr}$ in $\mathrm{Ar}$, wobei durch frühere Fragmentierungsanalyse-Messungen im Quadrupolmassenspektrometer sichergestellt ist, daß sich höchstens ein $\mathrm{HBr}$ Molekül in einem Ar Cluster befindet [Bau99a, Bau01c].

Die experimentellen Bedingungen entsprechen weitgehend denen der ersten drei Abschnitte aus Tab. 4.1. Die Abweichungen, die im wesentlichen aus der Koexpansion resultieren, sind in Tab. 4.2 aufgeführt. Die Polarisation des Dissoziationslasers beträgt $90^{\circ}$.

\begin{tabular}{|lrrrr|}
\hline Mittlere Ar Clustergröße & $\mathbf{1 3 9}$ & $\mathbf{2 3 0}$ & $\mathbf{2 9 0}$ & $\mathbf{4 5 0}$ \\
\hline \hline Konz. $\mathrm{HBr}$ in Ar [\%] & 0.1 & 0.2 & 0.2 & 0.05 \\
Expansionsdruck [bar] & 3.8 & 5.0 & 5.5 & 4.6 \\
Düsentemperatur [ $\left.{ }^{\circ} \mathrm{C}\right]$ & -44 & -60.2 & -64.2 & -101.2 \\
Verhältnis $R\left(\frac{\text { perfect caging }}{\text { direct exit }}\right)$ & 5.8 & 12.5 & 15.4 & 58.6 \\
\hline
\end{tabular}

Tabelle 4.2: Expansionsparameter, mittlere Ar Clustergrößen und das Verhältnis $R\left(\frac{\text { perfect caging }}{\text { direct exit }}\right)$ für die Koexpansion von $\mathrm{HBr}$ in Argon.

Die experimentellen Flugzeitspektren und die dazugehörigen Energieverteilungen für das Wasserstofffragment sind in Abb. 4.6 dargestellt. Das Ergebnis ist an der Entwicklung der Intensität, die mit free exit gekennzeichnet ist, bei wachsender Ar Clustergröße zu beobachten. Mit zunehmender Clustergröße sind immer weniger Wasserstofffragmente in der Lage, den Cluster zu verlassen. Sie werden zumindest teilweise abgebremst (delayed exit), der größte Teil sogar vollständig (perfect caging). Dies wird durch das Verhältnis $R$ als Quotient zwischen den Intensitäten für perfect caging und direct exit in Tab. 4.2 und Abb. 4.6 ausgedrückt. Die Ursache dieses Effekts, insbesondere unter Berücksichtigung der Ar Cluster Größenverteilung, wird in Abschnitt 5.1 erläutert.

\subsubsection{Photodissoziation von HBr Molekülen auf Ne Clustern}

Für die Präparierung von $\mathrm{HBr}$ Molekülen auf Ne Clustern unterschiedlicher Größe werden die Ne Cluster in der in Abschnitt 2.1.1 beschriebenen Quelle erzeugt. Mit ihr sind minimale Düsentemperaturen von $60 \mathrm{~K}$ erreichbar. Die HBr Moleküle werden mittels Pick-up an die Cluster gelagert.

Die experimentellen Parameter sind in Tabelle 4.3 zusammengestellt. Die Ergebnisse in Form von Flugzeitspektren und Energieverteilungen sind in Abb. 4.7 zu sehen. 
In der Gruppe von P. Jungwirth sind Berechnungen zur Photodissoziation von $\mathrm{HBr}$ Molekülen in und auf Ne Clustern $(n=146)$ durchgeführt worden. Wenn man diese mit einer früheren Messung unserer Gruppe mit ähnlicher Clustergröße vergleicht, in der $\mathrm{HBr}$ mittels Pick-up an den Ne Cluster gelagert ist, stellt man fest, daß das experimentelle Ergebnis mit der Berechnung zu $\mathrm{HBr}$ im Ne Cluster übereinstimmt [Sla02a, Bau01a]. Für größere Ne Cluster sind keine Berechnungen durchgeführt worden. Die Vermutung drängt sich auf, daß Ne Cluster in diesem Größenbereich flüssig sind. Dies verursacht beim Pick-up Prozeß ein Eindringen des $\mathrm{HBr}$ Moleküls in den Cluster.

\begin{tabular}{|lll|}
\hline - Düsendurchmesser der konischen Düse & $d^{*}$ & $40 \mu \mathrm{m}$ \\
- & $l_{d}$ & $2 \mathrm{~mm}$ \\
- Önge der konischen Düse & $\alpha_{D}$ & $20^{\circ}$ \\
- äquivalenter Düsendurchmesser & $d_{a q}$ & $167 \mu \mathrm{m}$ \\
- stoffspezifische Konstante für Ne & $K_{c h}$ & $185 \frac{\mathrm{K}^{2.2875} \mu \mathrm{m}^{0.85}}{\mathrm{mbar}}$ \\
- Düsentemperatur & $p$ & $8.0 \mathrm{bar}$ \\
- mittlere Clustergröße & $T_{D}$ & $-212.8 \mathrm{bis}-146^{\circ} \mathrm{C}$ \\
- Pick-up Zellendruck & $\langle n\rangle$ & $100-1600$ \\
- Dissoziationswellenlänge & $p_{p}$ & $4 \cdot 10^{-2} \mathrm{mbar}$ \\
- Laserenergie im TOF-Zentrum & $\lambda_{\mathrm{Diss}}$ & $243 \mathrm{~nm}$ \\
- Winkel zwischen Laserpolarisierung & $E_{L}$ & $1.54 \mathrm{~mJ}$ \\
& & \\
- und Detektorachse & $\alpha$ & $90^{\circ}$ \\
- WMTOFMS mit langer Driftstrecke & $D$ & $320.3 \mathrm{~mm}$ \\
- Bbzugsspannung & $U_{s}$ & $5.19 \mathrm{~V}$ \\
- Meschleunigungsspannung & $U_{d}$ & $24.81 \mathrm{~V}$ \\
\hline
\end{tabular}

Tabelle 4.3: Experimentelle Parameter zur Photodissoziation von $\mathrm{HBr}$ Molekülen adsorbiert an Ne Cluster: im oberen Teil die Strahldaten, im folgenden die Laserdaten und abschließend die Einstellungen des WMTOFMS.

Aus Experimenten von T. Möller ist bekannt, daß kleine Ne Cluster flüssig sind und im Bereich $200<\langle n\rangle<300$ ein Phasenübergang von flüssig nach fest stattfindet [Pie97]. Experimente in der Gruppe H. Haberland zeigen eine Korrelation zwischen der Schmelztemperatur eines Clusters und seiner Größe. Für kleine Cluster ist die Schmelztemperatur 
niedriger als für große. Die Schmelztemperatur für große Cluster konvergiert gegen die des Festkörpers [Sch98].

Für die Messungen im Rahmen dieser Arbeit bedeutet das, die Fragestellung zu bearbeiten, wie sich die Flugzeitspektren aus der Photodissoziation von $\mathrm{HBr}$ in Ne Clusterumgebungen mit der Clustergröße verändern. Dieser Zusammenhang ist in Abb. 4.7 dargestellt.

Eine Veränderung in der Form der Spektren ist nicht erkennbar. Einzig die Intensität nimmt für große Cluster zu. Wenn ein Phasenübergang stattfinden würde, müßte sich dieser in einer Intensitätserhöhung bei der Energie des freien Ausgangs der Wasserstofffragmente $(1.3 \mathrm{eV})$ bemerkbar machen. Die $\mathrm{HBr}$ Moleküle befänden sich dann zumindest teilweise auf der Oberfläche des Clusters, und es gelänge dementsprechend einer größeren Anzahl von Wasserstofffragmenten, die Clusterumgebung ungehindert zu verlassen.

Erklärungen zur möglichen Position des $\mathrm{HBr}$ Moleküls in der Ne Clusterumgebung und die Beschaffenheit des Ne Clusters werden in Abschnitt 5.1 behandelt. Dazu werden Vergleiche mit den oben genannten Messungen und Rechnungen angestellt.

\subsection{Photodissoziation von $\mathrm{HCl}$ Molekülen in unterschiedlichen Clusterumgebungen}

Erstmals werden in dieser Arbeit Photodissoziationsexperimente von $\mathrm{HCl}$ Molekülen in Edelgasclusterumgebungen durchgeführt. Dabei wird die Photodissoziation von $\mathrm{HCl}$ adsobiert auf $\mathrm{Ne}, \mathrm{Ar}, \mathrm{Kr}$ und $\mathrm{Xe}$ Clustern studiert. Bei einigen Spezies wird zusätzlich die Größenabhängigkeit ermittelt. Experimente mit puren $\mathrm{HCl}$ Molekülen und $(\mathrm{HCl})_{x}-\mathrm{Ar}_{n}$ Mischclustern werden durch die Erzeugung in Koexpansion ermöglicht. Auch hier stellt sich wieder die Frage nach dem Einfluß des Aufenthaltsortes des $\mathrm{HCl}$ Moleküls in der Clusterumgebung auf die experimentellen Ergebnisse. $\mathrm{HCl}$ besitzt in der Photodissoziation des puren Moleküls wie die anderen Wasserstoffhalogenide eine deutliche Anisotropie. Bei der hier verwendeten Dissoziationswellenlänge von $193 \mathrm{~nm}$ sind die Übergänge, die in den Grundzustand $\mathrm{Cl}$ und den angeregten Zustand $\mathrm{Cl}^{*}$ führen, immer senkrecht. Die meisten Experimente dieses Abschnitts sind mit dem ArF Excimerlaser bei $193 \mathrm{~nm}$ ohne Vorzugspolarisierung durchgeführt worden. Einige Experimente mit Polarisierungen von 0 und $90^{\circ}$ zur Detektorachse ergeben Aufschluß über die Mechanismen der Photodissoziation in Clusterumgebungen. 

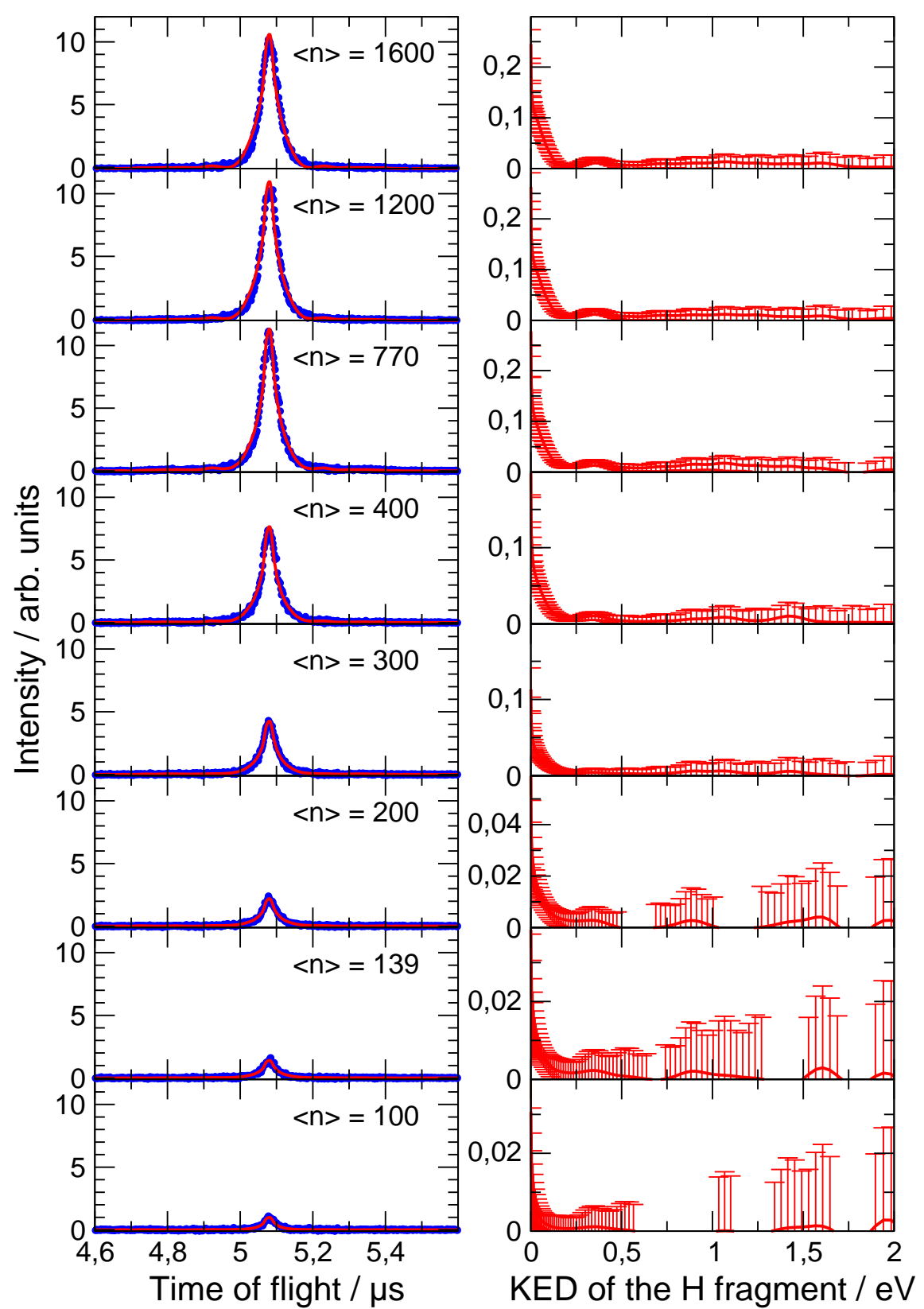

Abbildung 4.7: Photodissoziation von einem $\mathrm{HBr}$ Molekül auf Ne Clustern unterschiedlicher Größe. Gemessenes (•) und simuliertes (-) Flugzeitspektrum links und Energieverteilung rechts. Durch Variation von Düsentemperatur und Expansionsdruck werden Ne Cluster mit mittleren Clustergrößen $\langle n\rangle=100-1000$ erzeugt und durch pick-up mit einem HBr Molekül dotiert. Der Photodissoziationsprozeß zeigt abgesehen von der Gesamtintensität der nachgewiesenen Wasserstofffragmente keine Abhängigkeit von der Clustergröße. 


\begin{tabular}{|lrrrr|}
\hline Edelgas & Ne & Ar & Kr & Xe \\
\hline \hline Düsendurchmesser $[\mu \mathrm{m}]$ & 40 & 60 & 60 & 60 \\
Länge des Düsenkonus [mm] & & 2 & & \\
Öffnungswinkel des Düsenkonus $\left[^{\circ}\right]$ & 20 & 30 & 30 & 30 \\
Expansionsdruck [bar] & 8.0 & 5.0 & 5.0 & 5.0 \\
Düsentemperatur [ $\left.{ }^{\circ} \mathrm{C}\right]$ & -168.3 & -52.0 & -30.0 & -30.0 \\
mittlere Clustergröße & 200 & 200 & 370 & 1020 \\
\hline Abzugsspannung [V] & 5.19 & 8.6 & 8.6 & 8.6 \\
Beschleunigungsspannung [V] & 24.81 & 41.4 & 41.4 & 41.4 \\
MSP-Spannung [kV] & \multicolumn{5}{c}{3.5} \\
\hline
\end{tabular}

Tabelle 4.4: Strahldaten zur Erzeugung der Edelgascluster für Pick-up Experimente mit $\mathrm{HCl}$ und Spannungen im WMTOFMS (lang).

\subsubsection{Photodissoziation von $\mathrm{HCl}$ Molekülen auf $\mathrm{Ne}, \mathrm{Ar}, \mathrm{Kr}$, und $\mathrm{Xe}$ Clustern}

Dieser Abschnitt liefert einen Überblick über die Photodissoziation von $\mathrm{HCl}$ Molekülen, die mittels Pick-up an Edelgascluster ( $\mathrm{Ne}, \mathrm{Ar}, \mathrm{Kr}, \mathrm{Xe}$ ) angelagert sind. Dabei kommt der in Abschnitt 2.2.2 beschriebene ArF Excimerlaser bei einer Wellenlänge von $193 \mathrm{~nm}$ mit unpolarisierter Strahlung zum Einsatz. Die Pulsenergie im TOF-Zentrum beträgt $44 \mathrm{~mJ}$ bei einer Pulsdauer von $20 \mathrm{~ns}$. Die lonisation der Wasserstofffragmente findet weiterhin über den 2+1 REMPI Prozeß mit $243 \mathrm{~nm}$ (Polarisation $0^{\circ}, 1.54 \mathrm{~mJ} /$ Puls, $5 \mathrm{~ns}$ ) statt. Die Flugzeitspektren werden mit dem WMTOFMS in der längeren Ausführung (Abschnitt 2.3.3) aufgenommen. Die Anlagerung der $\mathrm{HCl}$ Moleküle an die Edelgascluster wird mittels Pick-up bei einem Zellendruck von $4 \cdot 10^{-2}$ mbar vorgenommen. Die Daten zur Erzeugung der verschiedenen Edelgascluster sind in Tab. 4.4 wiedergegeben. Probleme mit dem Auf||Iösungsvermögen und der Signalintensität verhinderten es, Edelgascluster mit identischer mittlerer Größe zu erzeugen und unter gleichen Bedingungen im WMTOFMS zu messen.

Die Energieverteilungen aus den Flugzeitspektren der Photodissoziation von $\mathrm{HCl}$ Molekülen adsorbiert an Edelgascluster sind in Abb. 4.8 zu sehen. Für alle vier Edelgase ist eine Dominanz des Käfigeffekts erkennbar. Die kinetische Überschußenergie eines Wasserstofffragments aus der Photodissoziation von $\mathrm{HCl}$ mit $193 \mathrm{~nm}$ liegt abhängig vom Produktkanal $\left(\mathrm{H}+\mathrm{Cl}\right.$ vs. $\left.\mathrm{H}+\mathrm{Cl}^{*}\right)$ bei 1.9 oder $2.0 \mathrm{eV}$. In diesem Bereich ist für $\mathrm{Ne}$ und $\mathrm{Ar}$ 
keine, für $\mathrm{Kr}$ und $\mathrm{Xe}$ nur eine sehr geringe Intensität mit breiter Verteilung zu erkennen. Im Gegensatz zu vergleichbaren Messungen der Photodissoziation von $\mathrm{HBr}$ und $\mathrm{HI}$ gelingt es hier den Wasserstofffragmenten nicht, den Cluster ungehindert zu verlassen (kein direct exit). Die Energieverteilungen aus der Photodissoziation von $\mathrm{HCl}$ adsorbiert an $\mathrm{Ne}, \mathrm{Ar}$ und $\mathrm{Kr}$ weisen nur vollständig abgebremste Wasserstofffragmente auf (perfect caging). Es gibt auch keine teilweise abgebremsten Fragmente mit verzögertem Austritt (delayed exit). Wasserstofffragmente mit dieser Eigenschaft liegen nur bei der Photodissoziation von $\mathrm{HCl}$ adsorbiert an Xe vor, wobei die Verteilung bei ungefähr $1 \mathrm{eV}$ schon fast abbricht und nicht kontinuierlich bis zur maximal verfügbaren Energie von $2 \mathrm{eV}$ abnimmt.

Die unterschiedlichen Clustergrößen spielen keine Rolle, wie insbesondere im folgenden Abschnitt 4.3.2 gezeigt wird.

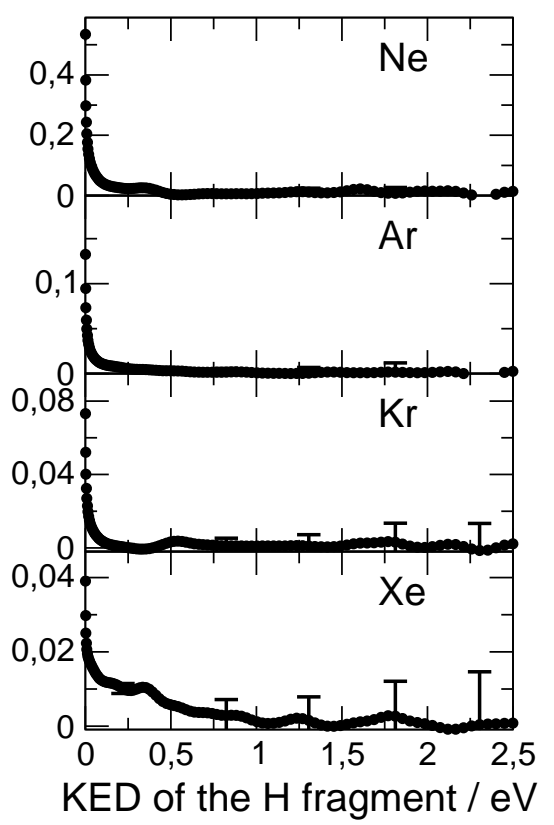

Abbildung 4.8: Energieverteilungen der Messungen $\mathrm{HCl}$ auf $\mathrm{Ne}_{n}, \mathrm{Ar}_{n}, \mathrm{Kr}_{\mathrm{n}}$ und $\mathrm{Xe}_{\mathrm{n}}$. Der Pick-up-Zellendruck von $\mathrm{HCl}$ beträgt $4 \cdot 10^{-2}$ mbar. Man beobachtet abgesehen von $\mathrm{Xe}_{\mathrm{n}}$ fast nur völlig abgebremste Wasserstofffragmente (caging, $E_{k i n}=0 \mathrm{eV}$ ). 


\subsubsection{Photodissoziation von $\mathrm{HCl}$ Molekülen auf $\mathrm{Ne}$ und Ar Clustern: Größenabhängigkeit}

Für den Käfigeffekt kann die Größe bzw. Ausdehnung der Umgebung je nach Aufenthaltsort des Chromophors eine zentrale Bedeutung besitzen. Im vorherigen Abschnitt stand die Art der Atome, aus denen die Cluster zusammengesetzt sind, im Fokus der Betrachtungen. In diesem Abschnitt ist das Augenmerk auf die Photodissoziation von $\mathrm{HCl}$ Molekülen adsorbiert an Ne und Ar Cluster mit mittleren Größen zwischen 100 und 1000 gerichtet.

\begin{tabular}{|lcccr|}
\hline Mittlere Ne Clustergröße & $\mathbf{1 0 0}$ & $\mathbf{1 4 7}$ & $\mathbf{2 0 0}$ & $\mathbf{8 0 0}$ \\
\hline \hline Expansionsdruck [bar] & \multicolumn{5}{c|}{8.0} \\
Düsentemperatur [ ${ }^{\circ} \mathrm{C}$ ] & -147.0 & $-159.0-168.3$ & -200.0 \\
\hline Abzugsspannung [V] & \multicolumn{5}{c}{5.19} \\
Beschl.-Spannung [V] & \multicolumn{5}{c}{24.81} \\
MSP-Spannung [kV] & 3.5 \\
\hline
\end{tabular}

Tabelle 4.5: Experimentelle Parameter zur Photodissoziation von $\mathrm{HCl}$ angelagert an $\mathrm{Ne}$ Cluster unterschiedlicher Größe

Wie schon besprochen findet bei der Photodissoziation von $\mathrm{HCl}$ in $\mathrm{Ne}$ und $\mathrm{Ar}$ Clusterumgebungen für die Wasserstofffragmente kein Austritt aus dem Käfig statt. Die Messungen, die in diesem Abschnitt vorgestellt werden, sollen zeigen, ob dieses Phänomen nicht nur nahezu unabhängig von der Art des Clusters, sondern auch von seiner Größe ist.

Die Flugzeitspektren und Energieverteilungen zur Photodissoziation von $\mathrm{HCl}$ Molekülen, die mittels Pick-up an Ne Cluster gelagert sind, sind in Abb. 4.9 zu sehen. Die zugehörigen experimentellen Parameter stehen in Tab. 4.5. Die entsprechenden Daten für Ar Cluster befinden sich in Abb. 4.10 und Tab. 4.6. Die weiteren experimentellen Bedingungen sind identisch mit den in Abschnitt 4.3.1 beschriebenen.

Die Spektren bei Variation der Ar und Ne Clustergrößen zeigen keine Abhängigkeit, die für große oder kleine Cluster einen direkten Austritt der Wasserstofffragmnete erwarten läßt. Für große Cluster (Ne: $\langle n\rangle=800 ; \operatorname{Ar}:\langle n\rangle=600$ und 1000) nimmt der Anteil teilweise abgebremster Wasserstofffragmente leicht zu. Für Ne erstreckt sich die Verteilung bis ca. $0.8 \mathrm{eV}$ und für $\mathrm{Ar}$ bis $0.5 \mathrm{eV}$.

Im Flugzeitspektrum der Photodissoziation von $\mathrm{HCl}$ auf $\mathrm{Ne}_{800}$ Clustern fällt im vorderen Teil eine asymmetrische Intensitätserhöhung auf. In anderen Messungen wie $\mathrm{HI}$ und $\mathrm{HCl}$ auf 


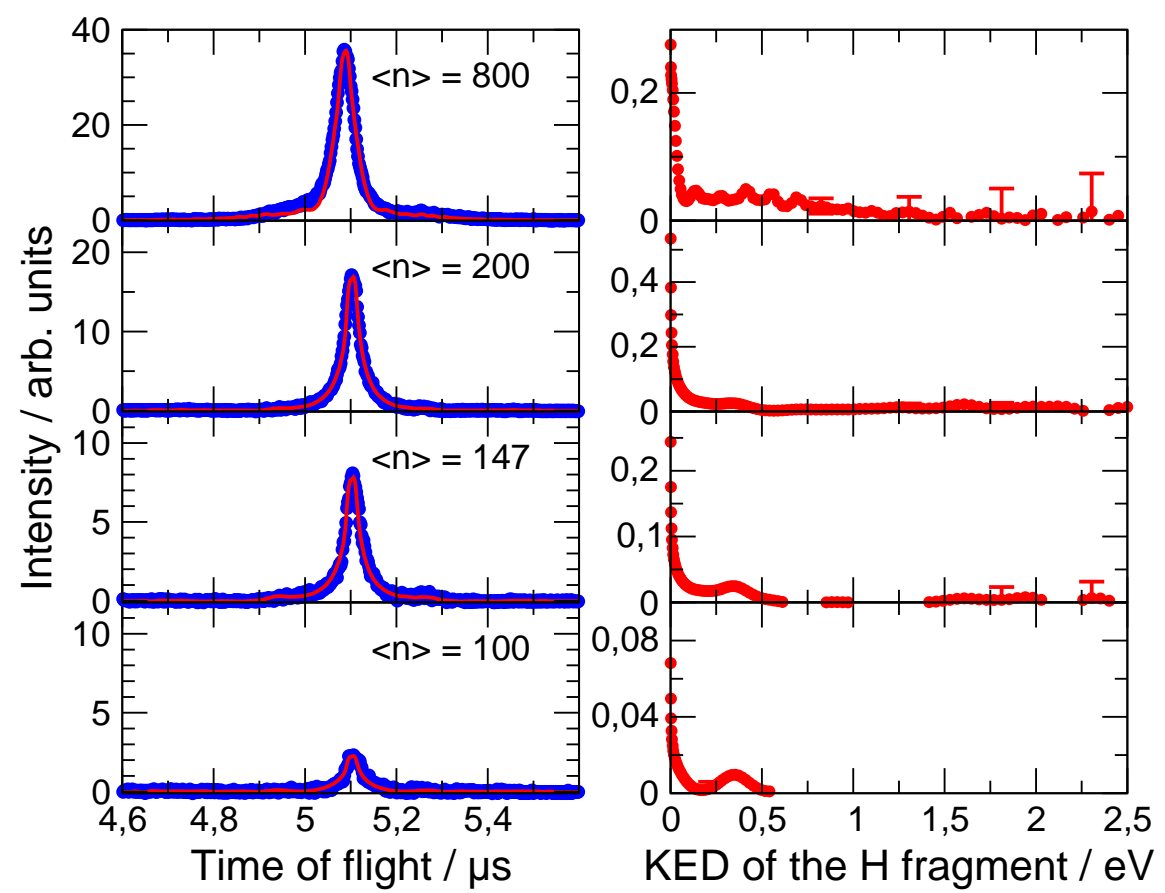

Abbildung 4.9: Größenabhängigkeit der Photodissoziation von $\mathrm{HCl}$ auf $\mathrm{Ne}_{\mathrm{n}}$ Clustern, $\langle n\rangle=100-800$. Gemessenes $(\bullet)$ und simuliertes $(-)$ Flugzeitspektrum links und Energieverteilung rechts. Für $\langle n\rangle=800$ ist im vorderen Teil des Spektrums eine Asymmetrie erkennbar.

\begin{tabular}{|lccccc|}
\hline Mittlere Ar Clustergröße & $\mathbf{1 4 0}$ & $\mathbf{2 0 0}$ & $\mathbf{3 0 0}$ & $\mathbf{6 0 0}$ & $\mathbf{1 0 0 0}$ \\
\hline \hline Düsendurchmesser [ $\mu \mathrm{m}]$ & 60 & & 40 & \\
Länge des Düsenkonus [mm] & \multicolumn{2}{c}{2} & & & \\
Öffnungswinkel des Düsenkonus [ $\left.{ }^{\circ}\right]$ & 30 & & 20 & \\
Expansionsdruck [bar] & 4.0 & 5.0 & 2.0 & 3.0 & 4.1 \\
Düsentemperatur [ [C] & -52.0 & & -140.0 & \\
\hline Abzugsspannung [V] & 8.6 & & 5.19 & \\
Beschl.-Spannung [V] & 41.4 & & 24.81 & \\
MSP-Spannung [kV] & & 3.5 & & \\
\hline
\end{tabular}

Tabelle 4.6: Experimentelle Parameter zur Photodissoziation von $\mathrm{HCl}$ angelagert an $\mathrm{Ar}$ Cluster unterschiedlicher Größe 

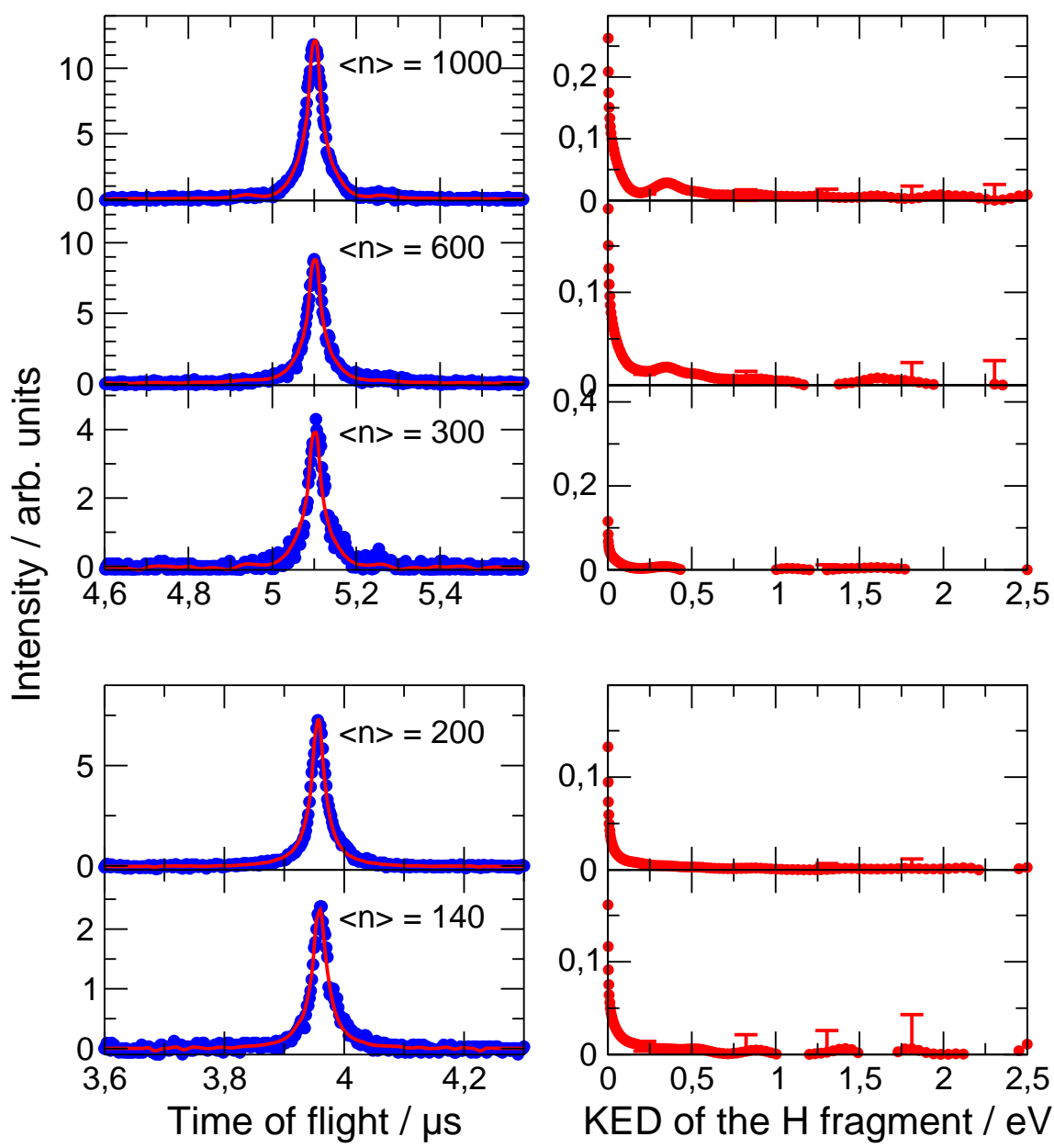

Abbildung 4.10: Größenabhängigkeit der Photodissoziation von $\mathrm{HCl}$ auf $\mathrm{Ar}_{\mathrm{n}} \mathrm{Clu}$ stern, $\langle n\rangle=140-1000$. Gemessenes $(\bullet)$ und simuliertes $(-)$ Flugzeitspektrum links und Energieverteilung rechts. Die unterschiedlichen Skalierungen im Flugzeitraum sind auf die verwendeten Clusterquellen und die daraus resultierenden Signalintensitäten zurückzuführen. Bei niedrigem Signal müssen die Abzugsspannungen erhöht werden. Dies führt zu einer früheren Ankunft der Fragmente. Die Messungen für Ar Cluster mit $\langle n\rangle \geq 300$ werden mit der in Abschnitt 2.1.1 beschriebenen Quelle (gekühlt mit Helium-Kompressor) durchgeführt, die übrigen mit der herkömmlichen Quelle (gekühlt mit flüssigem Stickstoff). Weitere Daten in Tab. 4.6. 
großen Xe Clustern ist dieser Effekt deutlich stärker ausgeprägt und wird der Bildung und Orientierung von $\mathrm{HXel}$ und $\mathrm{HXeCl}$ zugeschrieben (vgl. Abschnitt 4.4). Gegen die Bildung des Moleküls $\mathrm{HNeCl}$ spricht, daß es nach theoretischen Berechnungen nicht stabil ist.

Weitere Besonderheiten sind in den Flugzeitspektren zur Photodissoziation von $\mathrm{HCl}$ auf Ne und Ar Clustern nicht erkennbar. Die Gesamtintensität nimmt mit der Clustergröße zu, die Spektren werden von perfect caging dominiert, der delayed exit Anteil nimmt mit der Clustergröße zu. Freien Austritt direct exit aus der Clusterumgebung gibt es nicht.

\subsubsection{Photodissoziation von $\mathrm{HCl}$ Clustern und $(\mathrm{HCl})_{x} \mathrm{Ar}_{n}$ Mischclustern}

Die Koexpansion von $\mathrm{HCl}$ in Ar führt je nach den äußeren Bedingungen zu einem reinen $\mathrm{HCl}$ Molekularstrahl, zu einem $\mathrm{HCl}$ Clusterstrahl oder zu einem Strahl aus Mischclustern, die sich aus $\mathrm{Ar}$ und $\mathrm{HCl}$ zusammensetzen. Bei diesen Mischclustern befindet sich der $\mathrm{HCl}$ Cluster im Ar Cluster. Die Wahl der Parameter erlaubt auch die Platzierung von nur einem $\mathrm{HCl}$ Molekül in einem Ar Cluster.

Für die hier vorgestellten Messungen wird eine Mischung von $3 \% \mathrm{HCl}$ in $\mathrm{Ar}$ expandiert. Mit den gewählten Bedingungen befinden sich mehrere $\mathrm{HCl}$ Moleküle in einem $\mathrm{Ar}$ Cluster [Bau99a, Bau01c]. Pure $\mathrm{HCl}$ Cluster lassen sich mit dieser Mischung bei hohen Temperaturen (über $300 \mathrm{~K}$ ) erzeugen.

Bei der Erzeugung von $(\mathrm{HCl})_{x} \mathrm{Ar}_{n}$ Mischclustern wird die Bindungsenergie des $(\mathrm{HCl})_{x}$ Clusters, der sich im Ar Cluster befindet, an diesen abgegeben. Dies führt zum Abdampfen von Ar Atomen. Die Bindungsenergie eines Ar Atoms an den Cluster beträgt größenabhängig zwischen 15 und $40 \mathrm{meV}$ (Dimer: $10 \mathrm{meV}$, Festkörper: $80 \mathrm{meV}$ ). Die Bindungsenergie eines $\mathrm{HCl}$ Moleküls an ein $\mathrm{HCl}_{x}$ Tetramer liegt bei ungefähr $85 \mathrm{meV}$ [Lat97].

Die Anzahl der $\mathrm{HCl}$ Moleküle in den puren $\mathrm{HCl}_{\mathrm{m}}$ Clustern und den Mischclustern liegt zwischen sechs und sieben [Bau97a, Bau97b, Bau95], was auf der Grundlage von Fragmentierungsanalysen zu $\mathrm{HBr}$ Clustern aus der Koexpansion mit $\mathrm{Ar}$ abgeschätzt wurde. Es werden zwischen 500 und $600 \mathrm{meV}$ Bindungsenergie des $\mathrm{HCl}_{7}$ Clusters an den Ar Clusterkäfig abgegeben, was zum Abdampfen von 13-40 Ar Atomen führt. In Tab. 4.7 sind die Ar Clustergrößen für die in diesem Abschnitt vorgestellten Messungen aufgelistet. Die Messungen sind wie in Abb. 4.11 von (1) - (6) durchnummeriert. Die Düsentemperaturen und Expansionsdrücke sind ebenfalls in der Tabelle aufgeführt. Mit $\langle n\rangle_{\text {pur }}$ wird die mittlere Clustergröße der Ar Cluster für eine pure Expansion von Ar bei diesen Bedingungen bezeichnet. $E_{\text {Bind }}(\mathrm{Ar})$ gibt die Bindungsenergie pro Ar Atom für einen Ar Cluster der 
Größe $\langle n\rangle_{\text {pur }}$ an. In der letzten Spalte ist mit $\langle n\rangle_{\text {Misch }}$ die mittlere Anzahl von Ar Atomen in einem $(\mathrm{HCl})_{x} \mathrm{Ar}_{n}$ Mischcluster angegeben, der durch die Bindungsenergie von $(\mathrm{HCl})_{\mathrm{x}}$, $\langle x\rangle=6-7$, einen Teil der Ar Atome verloren hat $\left(\langle n\rangle_{\text {pur }}-\langle n\rangle_{\text {Misch }}\right)$.

\begin{tabular}{|cccccc|}
\hline Messung & $T[\mathrm{~K}]$ & $p[\mathrm{bar}]$ & $\langle n\rangle_{\text {pur }}$ & $E_{\text {Bind }}(\mathrm{Ar})[\mathrm{eV}]$ & $\langle n\rangle_{\text {Misch }}$ \\
\hline \hline$(1)$ & 221 & 5.0 & 200 & 40 & 185 \\
$(2)$ & 221 & 3.7 & 122 & 28 & 100 \\
$(3)$ & 233 & 3.7 & 100 & 25 & 76 \\
$(4)$ & 253 & 3.7 & 75 & 22 & 48 \\
$(5)$ & 273 & 3.7 & 55 & 19 & 23 \\
$(6)$ & 313 & 3.8 & 32 & 15 & 0 \\
\hline
\end{tabular}

Tabelle 4.7: $(\mathrm{HCl})_{x} \mathrm{Ar}_{n}$ Mischcluster werden in der Koexpansion einer $3 \% \mathrm{HCl}$ in Ar Mischung erzeugt. Die Bindungsenergie des in den Ar Cluster eingebetteten $\mathrm{HCl}$ Clusters führt zum Abdampfen von $\mathrm{Ar}$ Atomen. Aus der mittleren $\mathrm{HCl}$ Clustergröße von $\langle n\rangle=7$ resultiert eine Bindungsenergie von ca. $600 \mathrm{meV}$ [Lat97]. Die Bindungsenergie für ein Ar Atom im Cluster $E_{\text {Bind }}(\mathrm{Ar})$ ist oben aufgeführt, ebenso die Düsentemperatur $T$, der Expansionsdruck $p$, die zu erwartende Clustergröße für pure Ar Cluster $\langle n\rangle_{\text {pur }}$ und die berechnete Anzahl von Ar Atomen im $(\mathrm{HCl})_{\times} \mathrm{Ar}_{n}$ Mischcluster

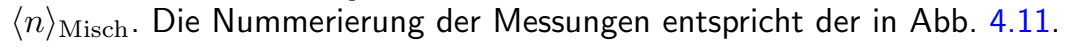

Die Messungen für die Mischcluster sind in Abb.4.11 dargestellt [(1)-(5)], auf der linken Seite die Flugzeitspektren, auf der rechten die zugehörigen Energieverteilungen. Der Käfigeffekt gewinnt mit wachsender Ar Clustergröße an Bedeutung. Messung (6) zeigt die Ergebnisse für pure $\mathrm{HCl}_{m}$ Cluster mit einer mittleren Clustergröße von $\langle m\rangle=6$. Strukturrechnungen für reine $\mathrm{HCl}$ Cluster sind in Abschnitt 5.1.6 aufgeführt.

Bei der Messung reiner $(\mathrm{HCl})_{6}$ Cluster werden keine völlig abgebremsten Wasserstofffragmente detektiert. Die Intensitätsverteilung der direct exit Fragmente erstreckt sich im Energieraum über einen Bereich von 1.7-2.0 eV (Auf|lösungsbegrenzung). Allerdings liegt die gemessene Untergrenze für die kinetische Energie der Wasserstofffragmente bei $1.5 \mathrm{eV}$. Damit gibt es einige Fragmente, die zumindest ein- oder zweimal mit anderen Molekülen im Cluster stoßen, bevor sie diesen verlassen.

Für die $\mathrm{HCl}$ Cluster in Ar Clustern verändert sich die Intensitätsverteilung im Bereich zwischen 1.5 und $2.0 \mathrm{eV}$ nicht. Schon für einen Ar Käfig mit 23 Atomen (Messung 5) ist ein großer Anteil von vollständig abgebremsten Wasserstofffragmenten erkennbar. Dieser Anteil steigert sich bis zur Clustergröße $\langle n\rangle=185$ soweit, daß er größere Intensität als der direkte Austritt besitzt. Zu größeren Clustern hin gibt es zwar einige Wasserstofffragmente 

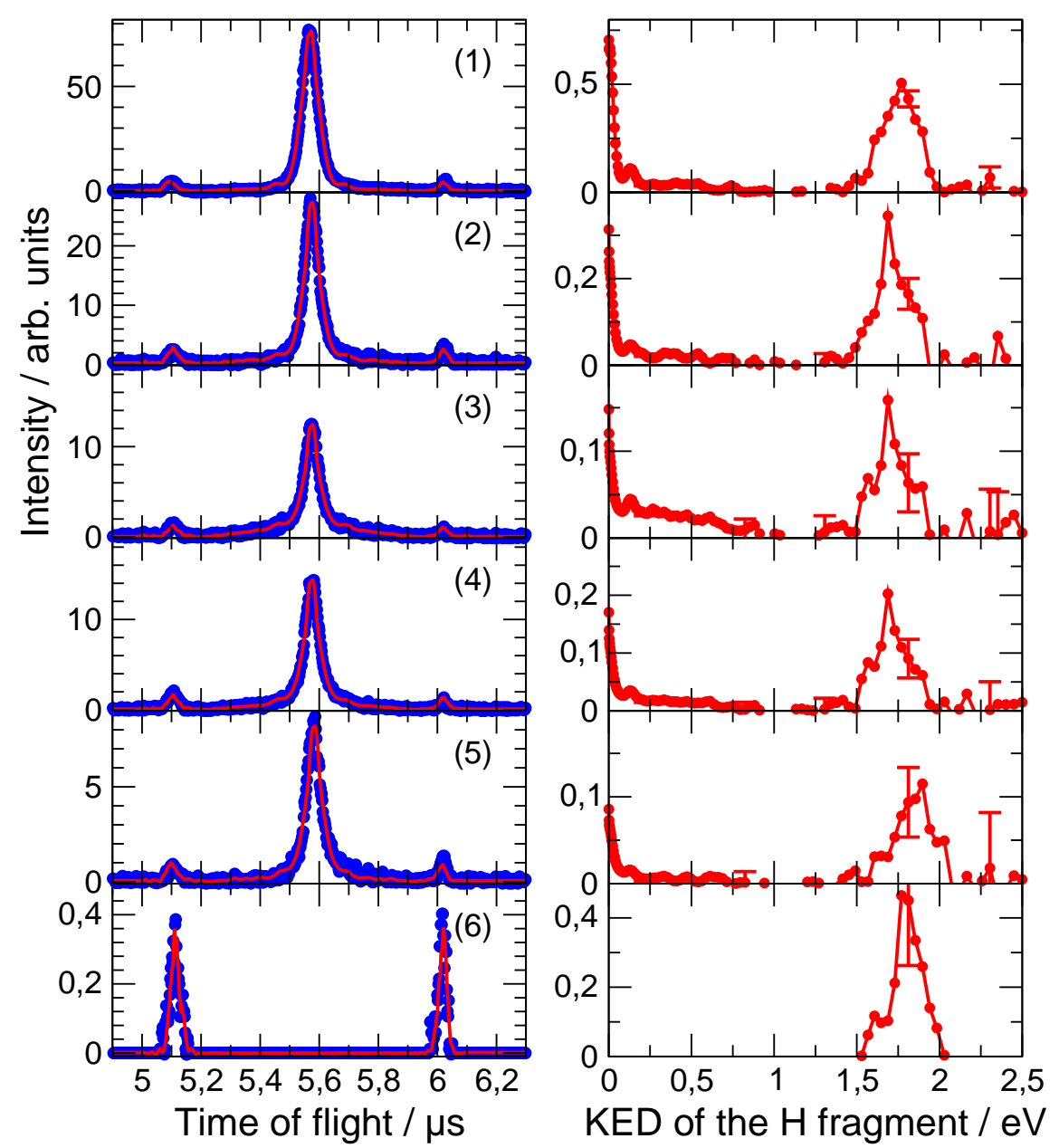

Abbildung 4.11: Photodissoziation von $(\mathrm{HCl})_{\mathrm{x}}-\mathrm{Ar}_{\mathrm{n}},\langle x\rangle \approx 6-7,\langle n\rangle=0-185$. Gemessenes (•) und simuliertes (-) Flugzeitspektrum links und Energieverteilung rechts. Die Cluster werden mittels Koexpansion einer Mischung von $3 \% \mathrm{HCl}$ in $\mathrm{Ar}$ erzeugt. Dissoziationslaser 193 nm, $50.7 \mathrm{~mJ} /$ Puls, unpolarisiert. Ionisationslaser $243 \mathrm{~nm}$, $1.54 \mathrm{~mJ} /$ Puls, Polarisation $0^{\circ}$ zur Detektorachse. Strahldaten in Tab. 4.7, Clusterzusammensetzung und Daten zum Käfigeffekt Tab. 4.8.

die teilweise abgebremst werden (Intensität zwischen 0.3 und $1.0 \mathrm{eV}$ ), dieser Effekt des delayed exit spielt aber fast keine Rolle. Der Energiebereich von 1.0 bis $1.5 \mathrm{eV}$ bleibt vollständig unbesetzt. Die Anteile von $\mathrm{HCl}$ Molekülen und $\mathrm{Ar}$ Atomen in den Clustern sowie die Bedeutung der Anteile im Käfigeffekt sind in Tab. 4.8 nachzulesen.

Abbildung 4.12 zeigt eine Messung, die mit den Bedingungen von Messung (2) aus Abb. 


\begin{tabular}{|ccc|}
\hline Messung & Cluster & $R\left(\frac{\text { perfect caging }}{\text { direct exit }}\right)$ \\
\hline \hline$(1)$ & $(\mathrm{HCl})_{7} \mathrm{Ar}_{185}$ & 1.4 \\
$(2)$ & $(\mathrm{HCl})_{7} \mathrm{Ar}_{100}$ & 0.9 \\
$(3)$ & $(\mathrm{HCl})_{7} \mathrm{Ar}_{76}$ & 0.9 \\
$(4)$ & $(\mathrm{HCl})_{7} \mathrm{Ar}_{48}$ & 0.8 \\
$(5)$ & $(\mathrm{HCl})_{7} \mathrm{Ar}_{23}$ & 0.7 \\
$(6)$ & $(\mathrm{HCl})_{6}$ & 0.0 \\
\hline
\end{tabular}

Tabelle 4.8: Der Anteil völlig abgebremster Wasserstofffragmente perfect caging im Verhältnis zu den Fragmenten, die den Cluster ungehindert verlassen direct exit wird für die Messungen aus Abb. 4.11 mit $R\left(\frac{\text { perfect caging }}{\text { direct exit }}\right)$ angegeben.

4.11 identisch ist, nun allerdings mit polarisiertem Dissoziationslaser. Die Polarisierung der $193 \mathrm{~nm}$ Strahlung erfolgt mit einem Dünnschichtplattenpolarisator, an dem ein großer Teil des Lichts durch Reflexion verlorengeht. Für die Photodissoziation stehen im TOF Zentrum ungefähr $7 \mathrm{~mJ}$ pro Puls zur Verfügung. Ansonsten sind die experimentellen Bedingungen mit den weiter oben in diesem Abschnitt beschriebenen identisch.
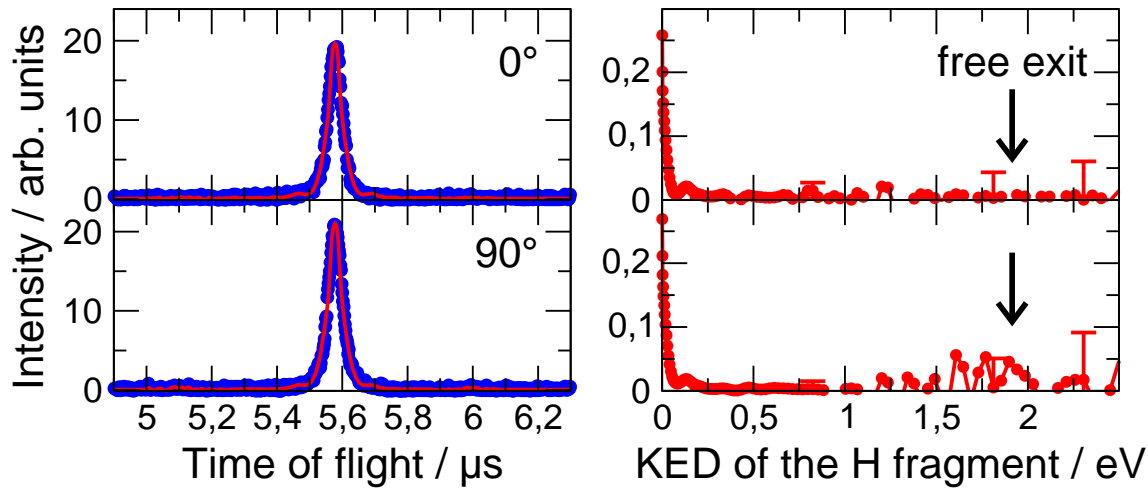

Abbildung 4.12: Photodissoziation von $(\mathrm{HCl})_{x}$ in $\mathrm{Ar}_{n}$ in Abhängigkeit von der Laserpolarisation, $\langle x\rangle=7,\langle n\rangle=100$, s. Abb. 4.11 Teil (2). Gemessenes (•) und simuliertes (-) Flugzeitspektrum links und Energieverteilung rechts. Der obere Teil zeigt die Messung bei einer Polarisation des Dissoziationslasers $(193 \mathrm{~nm})$ von $0^{\circ}$, der untere bei $90^{\circ}$ zur Detektorachse. Die Polarisierung erfolgt mit einem drehbaren Dünnschichtplattenpolarisator (TFP, s. Abschnitt 2.2.3). Das Licht hat eine Energie von $12 \mathrm{~mJ}$. Auf der rechten Seite bezeichnet free exit die Energie der Wasserstofffragmente, die den Cluster ungestört verlassen. 
Die Flugzeitspektren werden hier ganz klar von dem Mittelpeak dominiert. Fast alle Wasserstofffragmente sind vollständig abgebremst. Auch die Gesamtintensität in den Spektren ist fast identisch. Erst die Transformation in den Energieraum zeigt einen Unterschied im freien Ausgang von Wasserstofffragmenten. Die Messung unter einer Laserpolarisation von $90^{\circ}$ weist einen geringen direct exit Anteil auf. Einen Unterschied zwischen der $0^{\circ}$ und $90^{\circ}$ Messung sollte man aufgrund der Anisotropie in der Photodissoziation von $\mathrm{HCl}$ erwarten. Allerdings ist nicht klar, warum der Unterschied nicht deutlicher ausfällt. Wenn man diese Messungen mit Messung (2) aus Abb. 4.11 vergleicht sollte der freie Austritt unter $90^{\circ}$ (senkrechter Übergang, $\beta=-1$ ) zumindest genauso deutlich sein, wie bei dieser mit unpolarisiertem Licht.

\subsection{Bildung, Orientierung und Dissoziation von $\mathrm{HXel}$ und $\mathrm{HXeCl}$ Molekülen}

In diesem Abschnitt wird über die Erzeugung der ionisch gebundenen Moleküle $\mathrm{HXeCl}$ und $\mathrm{HXel}$ berichtet. Die Moleküle bilden sich bei der Photodissoziation von $\mathrm{HCl}$ und $\mathrm{HI}$ adsorbiert auf großen Xe Clustern. Sie werden über eine asymmetrische Verteilung in den Flugzeitspektren der Wasserstofffragmente identifiziert, die aus der Orientierung von $\mathrm{HXeCl}$ und $\mathrm{HXel}$ resultiert. Eine Kombination des starken gepulsten Laserfeldes und des schwachen elektrischen Feldes im WMTOFMS führt aufgrund der großen Anisotropie in der Polarisierbarkeit dieser Moleküle zu einer Orientierung (Abschnitt 1.4.2). Die asymmetrische Verteilung ist der symmetrischen Verteilung aus der ursprünglichen Photodissoziation von $\mathrm{HCl}$ oder $\mathrm{HI}$ überlagert, so daß sie zur quantitativen Auswertung der Spektren von dieser separiert werden muß. Die Bildung von $\mathrm{HXeX}(\mathrm{X}=\mathrm{Cl}, \mathrm{I})$ setzt dessen Bindungsenergie an den umgebenden $\mathrm{Xe}$ Cluster frei, der dabei vollständig verdampft. Unter Umständen können noch ein oder zwei Xe Atome an das Molekül HXeX angelagert sein, aber an sich ist das Molekül frei und kann in den Feldern ausgerichtet und orientiert werden.

\subsubsection{Energieverteilung der Dissoziation von $\mathrm{HXel}$ in Abhängigkeit vom Polarisationswinkel $\alpha$ und von der Feldrichtung im WMTOFMS}

Die Diskussion richtet sich auf die Photodissoziationsexperimente von $\mathrm{HI}$ auf großen $\mathrm{Xe}$ Clustern $107<\langle n\rangle<1000$. Zur Dissoziation kommt Laserstrahlung einer Wellenlänge von $243 \mathrm{~nm}$ bei einer Pulsenergie von $1.76 \mathrm{~mJ}$ im TOF Zentrum zum Einsatz. Die Pulsdauer 
des Lasers beträgt $5 \mathrm{~ns}$ und der Strahl wird mittels einer $400 \mathrm{~mm}$ Linse in das TOF Zentrum auf einen Durchmesser von $14 \mu \mathrm{m}$ fokussiert. Daraus resultiert eine Leistungsdichte von $2.2 \cdot 10^{11} \mathrm{~W} / \mathrm{cm}^{2}$, die aber aufgrund von Inhomogenitäten im Strahlprofil vorsichtig auf $1.1 \cdot 10^{11} \mathrm{~W} / \mathrm{cm}^{2}$ nach unten korrigiert wird.
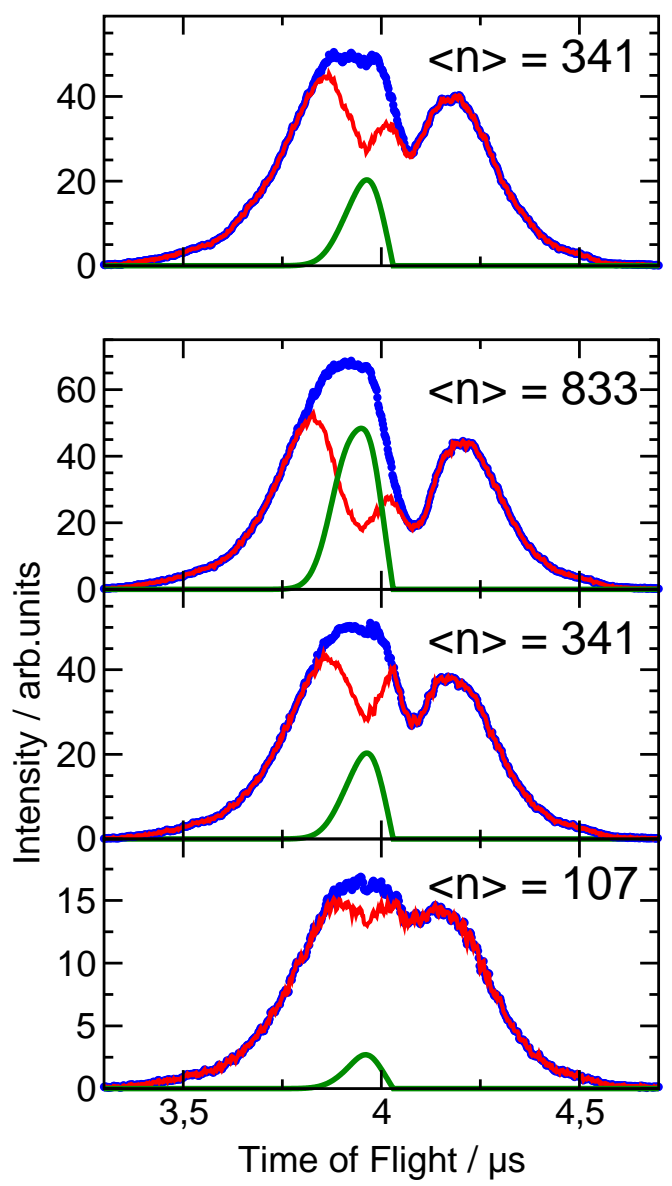

Abbildung 4.13: Flugzeitspektren für Wasserstofffragmente aus der Photodissoziation von $\mathrm{HI}$ auf Xe Clustern bei einer Dissoziationswellenlänge von $243 \mathrm{~nm}$. Die mittleren Clustergrößen $\langle n\rangle$ sind vermerkt. Die unteren drei Spektren sind bei einer Laserpolarisation von $90^{\circ}$, das obere bei $0^{\circ}$ aufgenommen worden. Die grauen Punkte umfassen das gemessene Spektrum, die schwarze Linie zeigt den symmetrischen, die gekreuzte den asymmetrischen Teil des Spektrums. Bei sämtlichen Messungen kommt die konische Düse mit einem min. Lochdurchmesser von $60 \mu \mathrm{m}$, einem Öffnungswinkel von $30^{\circ}$ bei einer Konuslänge von $2 \mathrm{~mm}$ zum Einsatz. Der Pick-up Zellendruck für $\mathrm{HI}$ beträgt $4 \cdot 10^{-2}$ mbar. Mittlere Clustergrößen, Düsentemperaturen und -drücke: $\langle n\rangle=107,220 \mathrm{~K}, 1.0$ bar; $\langle n\rangle=341,220 \mathrm{~K}, 2.03$ bar; $\langle n\rangle=833,220 \mathrm{~K}, 3.5$ bar. 
Es kommt das kurze WMTOFMS mit einer Abzugsspannung von 4.04 V zum Einsatz, was bei den geometrischen Gegebenheiten zu einer elektrischen Feldstärke von $4.2 \mathrm{~V} / \mathrm{cm}$ führt. Der Vektor der elektrischen Feldstärke ist parallel zur Detektorachse in Richtung des Detektors orientiert. Die Beschleunigungsspannung beträgt 12.29 V.

Es liegen experimentelle Ergebnisse mit einer Laserpolarisation von $0^{\circ}$ und $90^{\circ}$ zur Detektorachse / zum Vektor der elektrischen Feldstärke vor. Die Flugzeitspektren zur Photodissoziation von $\mathrm{HI}$ auf Xe Clustern der mittleren Größen $\langle n\rangle=107,341$ und 833 sind in Abb. 4.13 dargestellt. Die unteren drei Messungen sind bei einem Polarisationswinkel der Laserstrahlung von $90^{\circ}$ (aus [Bau99a]), die obere bei $0^{\circ}$ aufgenommen worden. Die Polarisationsrichtung zeigt unter diesen Bedingungen keinen Einfluß auf die Ergebnisse.

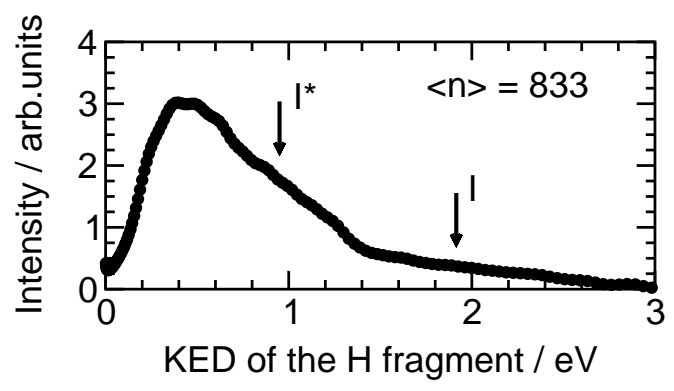

Abbildung 4.14: Energieverteilung der Wasserstofffragmente aus der Phtotodissoziation von $\mathrm{HI}$ auf Xe Clustern bei einer Dissoziationswellenlänge von $243 \mathrm{~nm}$. Die Energieverteilung stammt aus der Transformation des symmetrischen Teils des Flugzeitspektrums zur mittleren Clustergröße von $\langle n\rangle=833$ aus Abb. 4.13.

Die Spektren sind durch eine starke Asymmetrie gekennzeichnet, die für größere Cluster zunimmt. Diese Zunahme ist mit der höheren Wahrscheinlichkeit für HI-Teilchenfang in der Pick-up Zelle für größere Xe Cluster zu erklären. Dadurch befinden sich auf den größeren Xe Clustern mehrere HI Moleküle, was die Wahrscheinlichkeit zu Bildung von HXel erhöht. Die Asymmetrie kann nur von orientierten Molekülen herrühren, weil nicht orientierte Moleküle in diesem experimentellen Aufbau immer zu symmetrischen Flugzeitspektren führen [Bau97b, Bau00] (Abschnitt 3.1).

Aus diesem Grund läßt sich der asymmetrische Teil leicht aus den Flugzeitspektren extrahieren. Man zieht eine Verteilung ab, so daß das verbleibende Flugzeitspektrum symmetrisch ist. Damit lassen sich der vordere und hintere Teil des Spektrums in eine iden- 
tische Energieverteilung transformieren ${ }^{3}$. Diese Separation ist für die Flugzeitspektren in Abb. 4.13 ebenfalls zu sehen.

Die zu den Flugzeitspektren aus Abb. 4.13 korrespondierenden Energieverteilungen sind abgesehen von Intensitätsunterschieden fast identisch. Exemplarisch zeigt Abb. 4.14 die Energieverteilung des symmetrischen Teils des Flugzeitspektrums für eine Clustergröße von $\langle n\rangle=833$. Die Pfeile symbolisieren die kinetischen Anfangsenergien für Wasserstofffragmente aus der Photodissoziation von $\mathrm{HI}$ bei $243 \mathrm{~nm}$ mit I bzw. I* als Partnerfragment. Die Verteilung zeichnet sich durch eine Dominanz von teilweise abgebremsten Wasserstofffragmenten aus. Durch die Größe der Xe Atome im Verhältnis zu Ar oder $\mathrm{Ne}$ sind Stöße zwischen dem $\mathrm{H}$ Atom und einem Xe Atom deutlich wahrscheinlicher, allerdings ist der Energieverlust pro Stoß aufgrund der Impulsbilanz geringer. Dies führt zu einem Maximum der Verteilung bei einer kinetischen Energie von $0.4 \mathrm{eV}$ anstatt $0 \mathrm{eV}$.

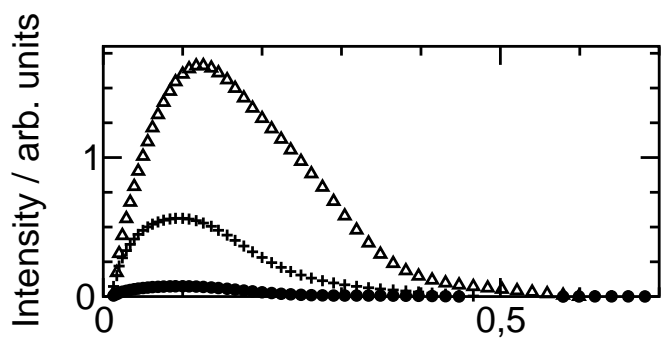

KED of the $\mathrm{H}$ fragment / eV

Abbildung 4.15: Wasserstofffragment-Energieverteilung aus der Dissoziation von orientiertem HXel. Die zugehörigen Xe Clustergrößen: $\langle n\rangle=833(\triangle), 341(+), 107$ $(\bullet)$.

Die Energieverteilungen für den asymmetrischen Teil der Flugzeitspektren sind in Abb. 4.15 dargestellt. Die vollständige Information steckt im vorderen Teil des Flugzeitspektrums, jedoch geht das Simulationsprogramm immer von symmetrischen Spektren aus. Daher wird der hintere Teil des Flugzeitspektrums für die langsamen Wasserstofffragmente künstlich erzeugt, und die symmetrische Verteilung anschließend in den Energieraum transformiert. Diese Ergänzung führt zu keiner Veränderung des Transformationsergebnisses.

\footnotetext{
${ }^{3}$ Symmetrie heißt in diesem Fall nicht, daß man das Spektrum in der Mitte bei Wasserstofffragmenten mit einer kinetischen Anfangsenergie von $0 \mathrm{eV}$ spiegeln kann. Der vordere und der hintere Teil des Spektrums unterscheiden sich leicht aufgrund der unterschiedlichen elektrischen Felder, die die Wasserstofffragmente mit verschiedener Anfangsrichtung sehen. Im hinteren Teil des Spektrums ist die Energie immer etwas niedriger und das Spektrum ist auf der Zeitachse komprimiert $\left(E_{\text {kin }} \propto \sqrt{t}\right)$.
} 
Die Energieverteilung derjenigen Wasserstofffragmente, die bevorzugt in Detektionsrichtung orientiert sind, erstreckt sich über einen Bereich von $0-0.4 \mathrm{eV}$ mit einem Maximum zwischen 100 und $120 \mathrm{meV}$.
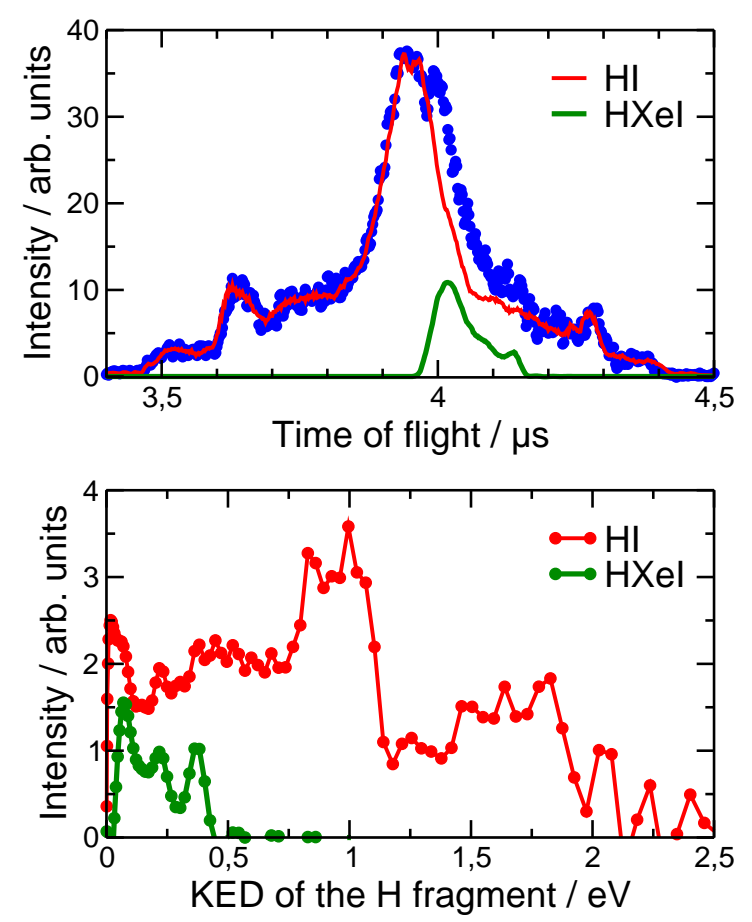

\begin{abstract}
Abbildung 4.16: Nachweis von HXel bei umgekehrter Feldrichtung im WM-TOFMS. Gemessenes $(\bullet)$ und simuliertes (-) Flugzeitspektrum (Strahldaten: Expansion 4 bar, $-40^{\circ} \mathrm{C}, \mathrm{Xe}_{\mathrm{n}},\langle n\rangle=835 ; 4 \cdot 10^{-2}$ mbar HI pick-up) im oberen Teil und die dazugehörige Energieverteilung im unteren Teil. Es liegt eine asymmetrische Verteilung vor, die sowohl im Flugzeit- als auch Energieraum separiert ist (jeweils die Kurve mit niedriger Intensität). Aufgrund der umgekehrten Feldrichtung ist im Vergleich zu Abb. 4.13 der asymmetrische Teil in den späten Teil des Flugzeitspektrums gewandert. Näheres zum geschalteten Abzugsfeld findet sich in Abschnitt 2.3.2
\end{abstract}

Ein weiteres Experiment zur Photodissoziation von $\mathrm{HI}$ auf $\mathrm{Xe}$ Clustern wurde durchgeführt, um die Existenz und Orientierung von HXel zu beweisen. Dafür kommt das in Abschnitt 2.3.2 beschriebene WMTOFMS in kurzer Ausführung aber mit geschaltetem Abzugsfeld zum Einsatz.

Die Idee dieses Experimentes ist folgende: Das Molekül wird durch den Laserpuls ausgerichtet, d. h. die Moleküle sind bei einer Laserpolarisation von $0^{\circ}$ parallel zur Detektorachse 
ausgerichtet. Ohne äußeres, statisches elektrisches Feld zeigen die Moleküle entweder mit dem Wasserstoffatom zum Detektor oder davon weg. Das elektrostatische Abzugsfeld im WMTOFMS sorgt in den oben beschriebenen Experimenten für eine Orientierung der Moleküle, so daß die Wasserstoffatome zum Detektor zeigen. Diese Orientierung ist durch das Dipolmoment des Moleküls und die elektrische Feldrichtung bedingt.

Wenn man nun das Abzugsfeld mit umgedrehter Feldrichtung anlegt, muß dies zu einer Orientierung der HXel Moleküle führen, bei der die Wasserstoffatome vom Detektor weg zeigen. Die Wasserstoffionen werden demnach im Anschluß an Dissoziation und lonisation entgegengesetzt der Detektorrichtung abgezogen werden. Sie würden, wenn die Beschaffenheit des elektrischen Feldes weiterhin so bestünde, auf die unterste Platte im WMTOFMS prallen.

Die Detektion der durch das umgedrehte Abzugsfeld vom Detektor weg fliegenden Wasserstoffionen ist dennoch möglich. Das Abzugsfeld mit umgedrehter Feldrichtung ist nur zur Orientierung der HXel Moleküle in der Zeitdauer des Laserpulses nötig. Anschließend wird die Richtung des Abzugsfeldes wieder in Richtung Detektor umgeschaltet. Ungefähr 50 ns nach dem Laserpuls herrscht ein stabiles statisches Abzugsfeld in Detektorrichtung, so daß alle Wasserstoffionen nachgewiesen werden können (s. Abb. 2.11).

Die ersten 50 ns nach der Dissoziation/Ionisation sorgen dafür, daß sich die Wasserstoffionen vom Startort (TOF-Zentrum) wegbewegen. Der Schwerpunkt ihrer räumlichen Verteilung bewegt sich dabei um $47 \mu \mathrm{m}$ vom Detektor weg. Die Teilchen mit einer maximalen Energie von $2.0 \mathrm{eV}$ legen in dieser Zeit feldfrei eine Strecke von $680 \mu \mathrm{m}$ zurück. Wenn das Feld umgeschaltet wird, befinden sich die Teilchen nicht mehr in dem relativ kleinen Anfangsvolumen, das durch den Strahlquerschnitts des Lasers von $14 \mu \mathrm{m}$ gegeben ist, sondern in einem Volumen, dessen Ausdehnung über einen Millimeter beträgt. Die Schwerpunktbewegung muß im Simulationsprogramm zur Transformation in eine Energieverteilung als veränderter Startort berücksichtigt werden. Das größere Anfangsvolumen führt praktisch zu keiner Änderung im Spektrum, weil das WMTOFMS unter Ortsfokussierungsbedingungen arbeitet.

Der vom Detektor weg verschobene Startort führt dazu, daß die Wasserstofffragmente den Detektor später erreichen. Das gesamte Flugzeitspektrum ist also zeitlich nach hinten verschoben. Ein direkter zeitlicher Vergleich mit den oben aufgeführten Messungen ist allerdings aufgrund unterschiedlicher TOF-Spannungen und des undefinierten elektrischen Feldes während der Umschaltzeit von 10 ns nicht möglich.

Die wesentliche Veränderung findet für Fragmente mit einer Vorzugsrichtung, die 
aus einer Orientierung resultiert, statt. Diese bekommen aufgrund des zuerst umgedrehten Abzugs-/Orientierungsfeldes eine Anfangsgeschwindigkeitskomponente entgegengesetzt zum Detektor. Dies bedeutet nach Umschalten des Abzugsfeldes, daß sie nun im hinteren Teil des Flugzeitspektrums nachgewiesen werden.

Resultiert die Asymmetrie bei den oben aufgeführten Experimenten tatsächlich aus der Bildung, Orientierung und Dissozation von HXel Molekülen, so muß sich diese Asymmetrie unter den gerade beschriebenen experimentellen Bedingungen in den hinteren Teil der Flugzeitspektren verlagern. Das Ergebnis dieser Messung ist in Abb. 4.16 zu sehen.

Die Form des Flugzeitspektrums (oberer Teil der Abb.) bestätigt das Vorhandensein einer Quelle von Wasserstofffragmenten mit einer Vorzugsrichtung. Auch hier sind die Meßdaten sowie der separierte asymmetrische und der symmetrische Teil des Spektrums zu sehen. Im unteren Teil der Abbildung sind die zugehörigen Energieverteilungen dargestellt.

Für den asymmetrischen Teil treffen ähnliche Eigenschaften wie für die vorher erwähnten Messungen zu. Das Maximum der Verteilung liegt bei $90 \mathrm{meV}$ und sie endet bei $0.4 \mathrm{eV}$. Allerdings ist eine deutliche Struktur zu erkennen, die auf Schwingungsanregungen des Moleküls HXel zurückzuführen sind [Lun00b].

Auch die deutlich besser aufgelöste Struktur in dem symmetrischen Teil des Spektrums, wo man bei 1.0 und $2.0 \mathrm{eV}$ den direct exit der Wasserstofffragmente beobachten kann, wirft Fragen zu den oben gezeigten Messungen auf. Ein weiterer Unterschied zu diesen ist die geringere Gesamt-Signalintensität und der kleinere asymmetrische Anteil.

Eine mögliche Erklärung ist der Unterschied in der Laserintensität. Diese ist bei den erst gezeigten Messungen um 30\% höher gewesen, was für die Orientierung von $\mathrm{HXel}$ eine wesentliche Rolle spielt. Das Lasersystem ist aufgrund seines Alters nicht mehr in der Lage, Leistung in diesem Bereich zu erzeugen, so daß die Messungen momentan nicht reproduziert werden können. Es gibt noch weitere Messungen zur Dissoziation von $\mathrm{HI}$ auf $\mathrm{Xe}$ Clustern bei Polarisationen von $0^{\circ}$ und $90^{\circ}$, die alle eine Asymmetrie im Flugzeitspektrum der Wasserstofffragmente zeigen, allerdings nicht so ausgeprägt. Bei allen ist auch die Struktur des Spektrums, die aus der Photodissoziation von $\mathrm{HI}$ resultiert, besser aufgelöst als in Abb. 4.13. Die schlechte Auf|lösung in den oben genannten Messungen ist vermutlich auf eine Dominanz der Dissoziation der orientierten HXel Moleküle zurückzuführen. Die Asymmetrie im Flugzeitspektrum taucht auch bei zuerst umgekehrtem Abzugsfeld sowohl bei einer Laserpolarisation von $0^{\circ}$ als auch von $90^{\circ}$ auf. 


\subsubsection{Energieverteilung der Dissoziation von $\mathrm{HXeCl}$ in Abhängigkeit vom Polarisationswinkel $\alpha$}

Nicht nur das Molekül HXel ist stabil und besitzt eine hohe Polarisierbarkeit, sondern auch die anderen $\mathrm{Xe}$ Verbindungen $\mathrm{HXeBr}$ und $\mathrm{HXeCl}$ zeichnen sich durch Stabilität und hohe Polarisierbarkeiten aus. Die Bindungsenergie nimmt von $\mathrm{HXel} z u \mathrm{HXeCl}$ zu. Mit den Polarisierbarkeiten verhält es sich umgekehrt (s. Tab. 1.4). Für den Nachweis von $\mathrm{HXeCl}$ bedeutet dies, daß es in unseren Experimenten auch gebildet werden kann. Es ist aber fraglich, ob es sich mit seiner geringeren Polarisierbarkeit in den kombinierten Feldern orientieren läßt, was Voraussetzung für einen erfolgreichen Nachweis ist.
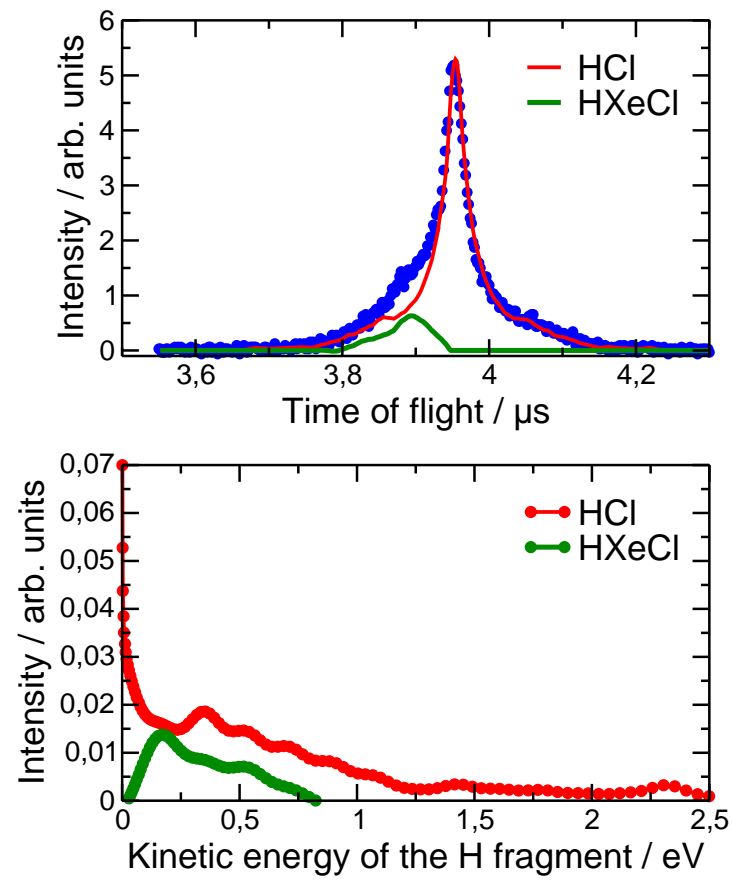

Abbildung 4.17: Photodissoziation von $\mathrm{HCl}$ auf $\mathrm{Xe}_{\mathrm{n}}$ : Polarisation $0^{\circ}$. Der obere Teil zeigt das gemessene $(\bullet)$ Flugzeitspektrum aus der Photodissoziation von $\mathrm{HCl}$ auf $\mathrm{Xe}_{\mathrm{n}}$ Clustern $(\langle n\rangle=1000)$ bei einer Dissoziationswellenlänge von $193 \mathrm{~nm}$, einer Pulsenergie von $12 \mathrm{~mJ}$ und einer Polarisation von $0^{\circ}$ zur Detektorachse. In der Simulation (oberer Teil) wird das Spektrum in einen symmetrischen Teil, der der Photodissoziation von $\mathrm{HCl}$ zugeordnet wird, und einen asymmetrischen Teil, der der Dissoziation von $\mathrm{HXeCl}$ zugeordnet wird, aufgeteilt. Entsprechend finden sich im unteren Teil des Bildes die zugehörigen Energieverteilungen. 
Bei den experimentellen Parametern gibt es Parallelen aber auch wesentliche Unterschiede zu den Versuchen mit $\mathrm{HI}$ und $\mathrm{Xe}$. Auch hier werden mittels Pick-up Technik $\mathrm{HCl}$ Moleküle an Xe Cluster aus der Düsenstrahlexpansion angelagert, der Dissoziationsprozeß und die Detektion weisen aber einige Unterschiede auf. So kommt zur Photodissoziation der $\mathrm{HCl}$ Moleküle der ArF Excimerlaser mit einer Wellenlänge von $193 \mathrm{~nm}$ und wahlweise einer Polarisation von $0^{\circ}$ oder $90^{\circ}$ zur Detektorachse zum Einsatz. Dieser weist zwar eine höhere Pulsenergie als die $243 \mathrm{~nm}$ Strahlung auf, die Leistungsdichte ist allerdings aufgrund der längeren Pulsdauer nahezu identisch. Im Idealfall erreicht man bei einer Pulsenergie von $12 \mathrm{~mJ}$ im TOF-Zentrum, einer Pulsdauer von $20 \mathrm{~ns}$ und einer Strahltaille im Fokus von $10 \mu \mathrm{m}$ eine Leistungsdichte, die bei $1.9 \cdot 10^{11} \mathrm{~W} / \mathrm{cm}^{2}$ liegt (bei $243 \mathrm{~nm}$ : $2.2 \cdot 10^{11} \mathrm{~W} / \mathrm{cm}^{2}$ ). Auch bei diesen Messungen wird die $243 \mathrm{~nm}$ Strahlung zur lonisation der Wasserstofffragmente benötigt. Sie ist parallel zur Detektorachse polarisiert $\left(0^{\circ}\right)$. Die Durchführung der Experimente geschieht mit dem neuen WMTOFMS mit längerer Driftstrecke. Dabei wird eine Abzugsspannung von $8.6 \mathrm{~V}$ und eine Beschleunigungsspannung von $41.4 \mathrm{~V}$ benutzt. Damit sind die Teilchen im Verhältnis zu den früheren Experimenten einer höheren elektrischen Feldstärke ausgesetzt $(7.8 \mathrm{~V} / \mathrm{cm}$ vs. $4.2 \mathrm{~V} / \mathrm{cm})$.

Diese Experimente liegen nur für eine Xe Clustergröße vor. Xenon Cluster mit einer mittleren Clustergröße von $\langle n\rangle=1000$ werden mit der im vorigen Abschnitt beschriebenen Clusterquelle bei einem Expansionsdruck von 5 bar und einer Düsentemperatur von $-30^{\circ} \mathrm{C}$ erzeugt. Der Pick-up Zellendruck für $\mathrm{HCl}$ beträgt $4 \cdot 10^{-2} \mathrm{mbar}$. Dies führt dazu, daß sich weniger $\mathrm{HCl}$ Moleküle (ein oder zwei) auf dem Xe Cluster befinden, als es für $\mathrm{HI}$ der Fall ist.

In Abb. 4.17 ist das Flugzeitspektrum der Wasserstofffragmente aus der Photodissoziation von $\mathrm{HCl}$ auf Xe Clustern bei einer Polarisation des Dissoziationslasers parallel zur Detektorachse zu sehen. Die Messung mit senkrechter Polarisation ist in Abb. 4.18 dargestellt. Die transformierten Energieverteilungen sind in den unteren Teilen der Abbildungen ebenfalls enthalten.

Es ist deutlich zu erkennen, daß das Spektrum bei einer Polarisation von $0^{\circ}$ eine Asymmetrie im vorderen Teil enthält, währenddessen das Flugzeitspektrum unter einem Polarisationswinkel von $90^{\circ}$ vollständig symmetrisch ist. Im ersten Fall wird der asymmetrische Teil separiert und sowohl im Flugzeitraum wie im Energieraum gesondert dargestellt. Sein Erscheinen wird der Bildung und Orientierung von $\mathrm{HXeCl}$ zugeschrieben.

Wenn man davon ausgeht, daß sich bei einer gemeinsamen Polarisation von $0^{\circ}$ die Felder von Dissoziations- und lonisationslaser addieren, liegt dort sogar eine größere Leistungs- 
dichte als bei den früheren Experimenten vor. Bei einer Polarisation des Dissoziationslasers von $90^{\circ}$ stehen die Laserfelder allerdings senkrecht aufeinander. Welchen Effekt dies genau hat, ist theoretisch noch nicht untersucht worden. Es ist eine wechselseitige, destruktive Beeinflussung der Felder zu erwarten, so daß keine Ausrichtung zustande kommt, die die Voraussetzung für die Orientierung im elektrostatischen Feld des WMTOFMS wäre.
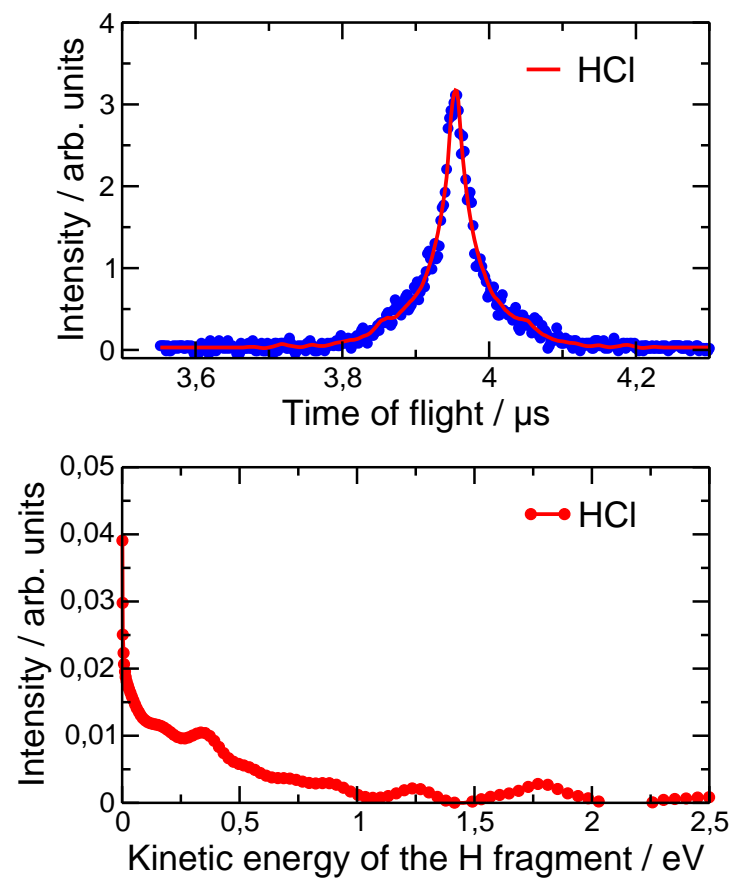

Abbildung 4.18: Photodissoziation von $\mathrm{HCl}$ auf $\mathrm{Xe}_{\mathrm{n}}$ : Polarisation $90^{\circ}$. Bedingungen identisch zu Abb. 4.17, abgesehen von der Polarisation des Dissoziationslasers $(193 \mathrm{~nm})$. Diese ist senkrecht zur Detektorachse $\left(90^{\circ}\right)$. Es ist keine Asymmetrie im Flugzeitspektrum zu erkennen. Eine Ausrichtung und Orientierung von $\mathrm{HXeCl}$ parallel zur Detektorachse findet nicht statt.

Welche Moleküle sich unter welchen Voraussetzungen in dieser Apparatur ausrichten und orientieren lassen, wird intensiv in Abschnitt 5.3 diskutiert. Dabei wird auch auf das Molekül $\mathrm{HXeBr}$ eingegangen, welches bezüglich seiner Eigenschaften zwischen $\mathrm{HXel}$ und $\mathrm{HXeCl}$ liegt. 


\section{Diskussion}

Die experimentellen Ergebnisse aus dem letzten Kapitel werden diskutiert und sowohl qualitativ als auch quantitativ mit theoretischen Untersuchungen verglichen. Dabei kommt der Position und möglichen Orientierung des Halogenwasserstoffmoleküls in der Clusterumgebung eine zentrale Rolle zu. Die Photodissoziation des freien Moleküls, im Gegensatz zum im Cluster eingebetteten, beleuchtet neue Aspekte der Anregungs- und Kopplungsmechanismen. Der Abschnitt über HRgX Moleküle zeigt, unter welchen Voraussetzungen Moleküle dieser Spezies gebildet und orientiert werden können. Die Unterschiede und Parallelen zu den vielfältigen Experimenten in Matrizen finden ebenfalls Eingang in die Betrachtungen.

\subsection{Position der HX Moleküle in der Edelgasclusterumgebung}

Der Käfigeffekt hat bei der Dissoziation von Molekülen in Clusterumgebungen Auswirkungen, die in der kondensierten Phase nicht zu beobachten sind. Im Cluster eröffnet sich den Dissoziationsfragmenten prinzipiell die Möglichkeit, den Cluster zu verlassen. In diesen Experimenten sind dies die Wasserstofffragmente aus der Photodissoziation von Halogenwasserstoffmolekülen. Ob diese Fragmente den Cluster verlassen können und wie hoch ihr Energieverlust durch Wechselwirkung mit dem Clusterkäfig ist, hängt zum einen von der Position des Moleküls in der Clusterumgebung ab, zum anderen aber auch von der Orientierung des Moleküls. Dabei ist zu beachten, ob überhaupt eine Vorzugsorientierung existiert und wenn ja, wie viele Clusterschalen das Wasserstofffragment bei einer bestimmten Orientierung bis zum Austritt durchqueren muß.

Die unterschiedlichen Möglichkeiten der Position und Orientierung des Moleküls in einem Cluster soll an Hand der Abb. 5.1 erklärt werden. Als Modellsystem dient ein Ar Cluster mit 147 Atomen, was drei vollständig abgeschlossenen Ikosaederschalen entspricht. 
Das Halogenwasserstoffmolekül ist größer als die Ar Atome aber auch punktförmig dargestellt, um keine Vorzugsorientierung zu suggerieren.

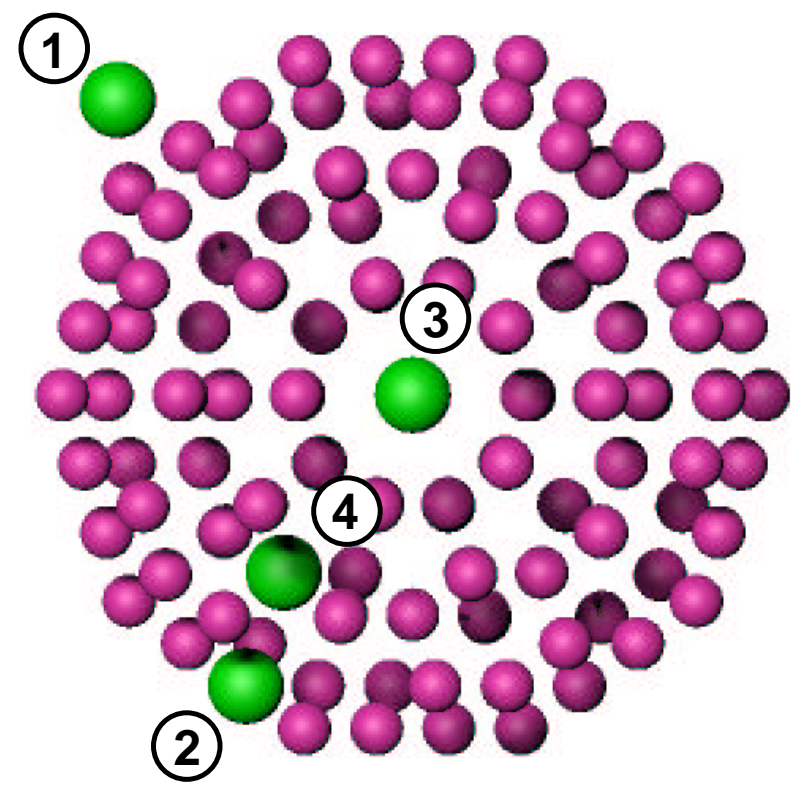

Abbildung 5.1: Mögliche Positionen eines Wasserstoffhalogenids in einem Edelgascluster

(1) Das Molekül lagert sich im Pick-up Prozeß an die Oberfläche des Clusters an. Für die Wechselwirkung zwischen Halogenwasserstoffmolekülen und Edelgasclustern führt dies für Clustergrößen $n \geq 5$ dazu, daß das Wasserstoffatom auf den Cluster zeigt. Die direkte Wechselwirkung erstreckt sich auf ein einzelnes Ar Atom im Cluster, so daß man einen Komplex X-H . . Ar betrachtet. Dabei kann das HX Molekül auf der Oberfläche verhinderte Rotationen (Libration) ausführen, die dazu führen, daß das Wasserstoffatom zeitweise parallel zur Clusteroberfläche zeigt [Sla01]. Für die Photodissoziation eröffnet dies den Wasserstofffragmenten die Möglichkeit, ohne jegliche Wechselwirkung die Clusterumgebung zu verlassen. Ein Wasserstoffatom, das während der Dissoziation auf den Cluster zeigt, muß diesen vollständig durchqueren, was normalerweise zum totalen Energieverlust führt. Die Energieverteilung der Wasserstofffragmente aus der Photodissoziation dieser Moleküle wird vom freien Austritt (direct exit) dominiert. Der Anteil abgebremster Fragmente ist gering. 
(2) Das HX Molekül ist beim Pick-up Verfahren in die Oberfläche des Ar Clusters eingebettet. Es nimmt in der ersten Schale die Position eines Clusteratoms ein. Das Molekül befindet sich in einer Zwangsanordnung. Bewegungen wie die oben genannten Librationen sind hier kleiner. Das Wasserstoffatom zeigt zum Zentrum des Clusters, was die Wechselwirkung mit dem Käfig deutlich erhöht. Fast alle Wasserstofffragmente aus der Dissoziation eines derartigen Moleküls werden vollständig abgebremst.

(3) Die Position des HX Moleküls im Zentrum des Ar Clusters resultiert aus der Koexpansion von $\mathrm{HBr}$ mit Ar. Anordnungen dieser Art lassen sich auch mittels Pick-up erreichen, wenn der Cluster anstatt fest flüssig ist. Dies führt zu nahezu ungehindertem Eindringen des Moleküls in den Cluster. Das Molekül nimmt die Minimumskonfiguration im Zentrum des Clusters ein [Niv99, Niv97]. Eine Vorzugsorientierung des Moleküls im Cluster existiert aufgrund der Symmetrie nicht. Das Wasserstofffragment muß zum Verlassen des Clusters immer dieselbe Anzahl von Schalen durchqueren. Dies trifft idealisiert nur für die magischen Clustergrößen und Isomere zu (s. Abb. 1.6 und Gl. 1.29). In diesen Experimenten werden Größen- und Isomerenverteilungen von Clustern erzeugt, die dazu führen, daß das Wasserstofffragment bei einer Clustergröße von $n=100$ je nach Orientierung zwei oder drei Clusterschalen sieht. In Abhängigkeit von der kinetischen Anfangsenergie können ab einer bestimmten Anzahl von Clusterschalen alle Wasserstofffragmente vollständig abgebremst werden. Diese Konfiguration führt zu Energieverteilungen, die von vollständig abgebremsten Fragmenten dominiert werden.

(4) Je nach Wechselwirkung zwischen Molekül und Clusteratomen sowie der Beschaffenheit der Clustergröße, kann ein Molekül beim Pick-up Prozeß auch tiefer in die Oberfläche des Clusters eindringen. In der zweiten Schale ist das Molekül vollständig von Clusteratomen umgeben. Die Wechselwirkung des Moleküls mit allen umgebenden Ar Atomen ist praktisch identisch. Es gibt also auch in diesem Fall keine Vorzugsorientierung. Wenn man sich die möglichen Orientierungen veranschaulicht, gibt es einen Winkelbereich, der ca. $20 \%$ des Raumes überdeckt, in dem das Wasserstofffragment aus der Dissoziation nur eine Clusterschale durchdringen muß, um den Käfig zu verlassen. Dies verursacht einen Anteil von direct exit Fragmenten im Spektrum. Ein weiterer Anteil von $20 \%$ von umgekehrt orientierten Molekülen muß fast den ganzen Cluster (vier Schalen) durchqueren, was zu vollständig abgebrem- 
sten Fragmenten führt. Die verbleibenden $60 \%$ müssen bei linearer Betrachtung zwei bis drei Clusterschalen hinter sich lassen, um den Käfig zu verlassen. Diese Fragmente werden nur teilweise abgebremst. Die Dissoziation der Moleküle, die in die zweite Schale des Clusters eingebaut sind, wird von teilweise abgebremsten Wasserstofffragmenten dominiert.

Der Moleküleinfang und die Einlagerung in den Cluster kann theoretisch berechnet werden. In einer Kooperation mit unserer Gruppe hat H. Vach Molekulardynamik Simulationen mit paarweisen 6-12 Lennard-Jones Potentialen ( $\mathrm{HX} \cdots \mathrm{Rg}$ und $\mathrm{XH} \cdots \mathrm{Rg}$ ) zum Pick-up Prozeß von $\mathrm{HI}$ und $\mathrm{HBr}$ an Ar Clustern durchgeführt. Die Ergebnisse sind in Abb. 5.2 aufgeführt [Vac02, Vac00, Vac99].
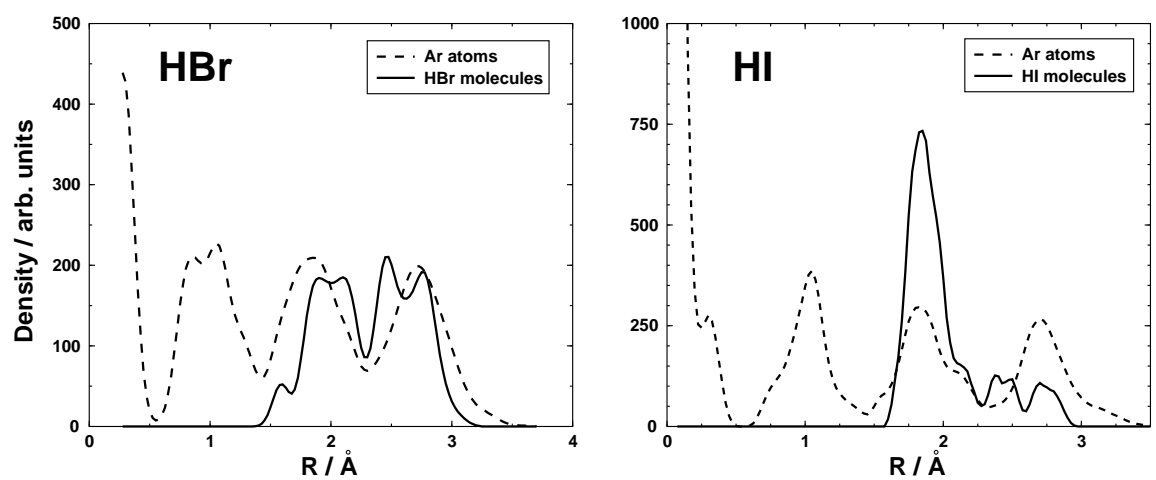

Abbildung 5.2: Aufenthaltswahrscheinlichkeit der Moleküle $\mathrm{HBr}$ und $\mathrm{HI}$ in $\mathrm{Ar}$ Clustern nach dem Einfang durch Pick-up. Angegeben ist in x-Richtung der Abstand vom Zentrum des Clusters. Es sind sowohl die Dichten für die Ar Atome als auch für die $\mathrm{HBr}$ und $\mathrm{HI}$ Moleküle angegeben [Vac02].

Aus beiden Rechnungen ist deutlich zu erkennen, daß keine $\mathrm{HBr}$ oder $\mathrm{HI}$ Moleküle direkt auf der Oberfläche adsorbiert werden. Im Mittel ist die Eindringtiefe für HI höher als für $\mathrm{HBr}$, was auf die unterschiedliche Wechselwirkung mit Ar zurückzuführen ist. Die Aufenthaltswahrscheinlichkeit für $\mathrm{HBr}$ ist nahezu gleichverteilt in der ersten und zweiten Clusterschale $^{1}$. Die HI Moleküle befinden sich zu ca. $80 \%$ in der zweiten Schale und nur zu $20 \%$ in der ersten Schale.

P. Slavíček aus der Gruppe von P. Jungwirth hat auf Basis dieser Ergebnisse die Energieverteilungen der Wasserstofffragmente aus der Photodissoziation von $\mathrm{HBr}$ und $\mathrm{HI}$ in

\footnotetext{
${ }^{1}$ Die Clusterschalen werden von außen nach innen gezählt.
} 
Ar Clustern berechnet. $\mathrm{Zu}$ den Anfangsbedingungen der Rechnungen zählt sowohl die Position und Orientierung des Moleküls im Cluster als auch der Zustand des Moleküls selbst. Im Grundzustand wird das HX-Ar Potential (3-body potential) benutzt. Für die Photoanregung werden die wichtigsten beteiligten Potentiale (nicht alle zwölf) nach der DIM-Methode (diatomics in molecules) berücksichtigt. Die Päparation des Grundzustandes geschieht vollständig quantenmechanisch mit Beteiligung der Libration. Die Wechselwirkung zwischen dem Wasserstofffragment nach der Dissoziation und den Ar Atomen wird mit ab initio Rechnungen behandelt.

\subsubsection{HBr Molekül in der Ar Clusteroberfläche}

Für $\mathrm{HBr}$ liegen Rechnungen getrennt nach den Anfangspositionen in der ersten und zweiten Schale vor [Sla02b]. In Abb. 5.3 ist der Vergleich zwischen Experiment und Theorie für die Photodissoziation von $\mathrm{HBr}$ Molekülen in einer Ar Clusterumgebung dargestellt. Die experimentellen Ergebnisse stammen aus dieser Arbeit und aus einer früheren Arbeit unserer Gruppe [Bau00].

Die Unterschiede in den experimentellen Ergebnissen konzentrieren sich auf den Bereich der teilweise abgebremsten Fragmente verursacht durch verschiedene Präparationsparameter (Temperatur, Druck) der Ar Cluster. Bei dem Experiment von 1999 war die Düsenund damit die Clustertemperatur höher, was die Aufenthaltswahrscheinlichkeit für das $\mathrm{HBr}$ Molekül in der zweiten Schale vergrößert (größere Eindringtiefe). Dies wiederum verursacht einen größeren Anteil von teilweise abgebremsten Wasserstofffragmenten.

Die Berechnungen mit unterschiedlichen Anfangspositionen des Moleküls, ermöglichen einen quantitativen Vergleich mit den experimentellen Ergebnissen. Für Moleküle in der ersten Schale gibt es wie vorhergesagt keinen direkten Käfigaustritt, für die in der zweiten Schale ist dagegen der Anteil ungehindert austretender Fragmente sehr groß. Eine Addition der beiden Rechnungen mit unterschiedlichen Anfangspostionen im Verhältnis 1:1 nach den Berechnungen von H. Vach (s. Abb. 5.2) zeigt quantitativ sehr gute Übereinstimmung mit den Meßergebnissen.

Unter diesem Aspekt sind auch die Messungen zur Photodissoziation von HBr Molekülen adsorbiert an Ar Cluster unterschiedlicher Temperatur aus dieser Arbeit zu betrachten (Abschnitt 4.2.1). Die Variation der Düsentemperatur ist nur schwach mit der Clustertemperatur korreliert. Trotzdem führt eine Erhöhung der Düsentemperatur zur Energiedissipation in den Cluster. Die Adsorption des $\mathrm{HBr}$ Moleküls an den Cluster ist mit einem 


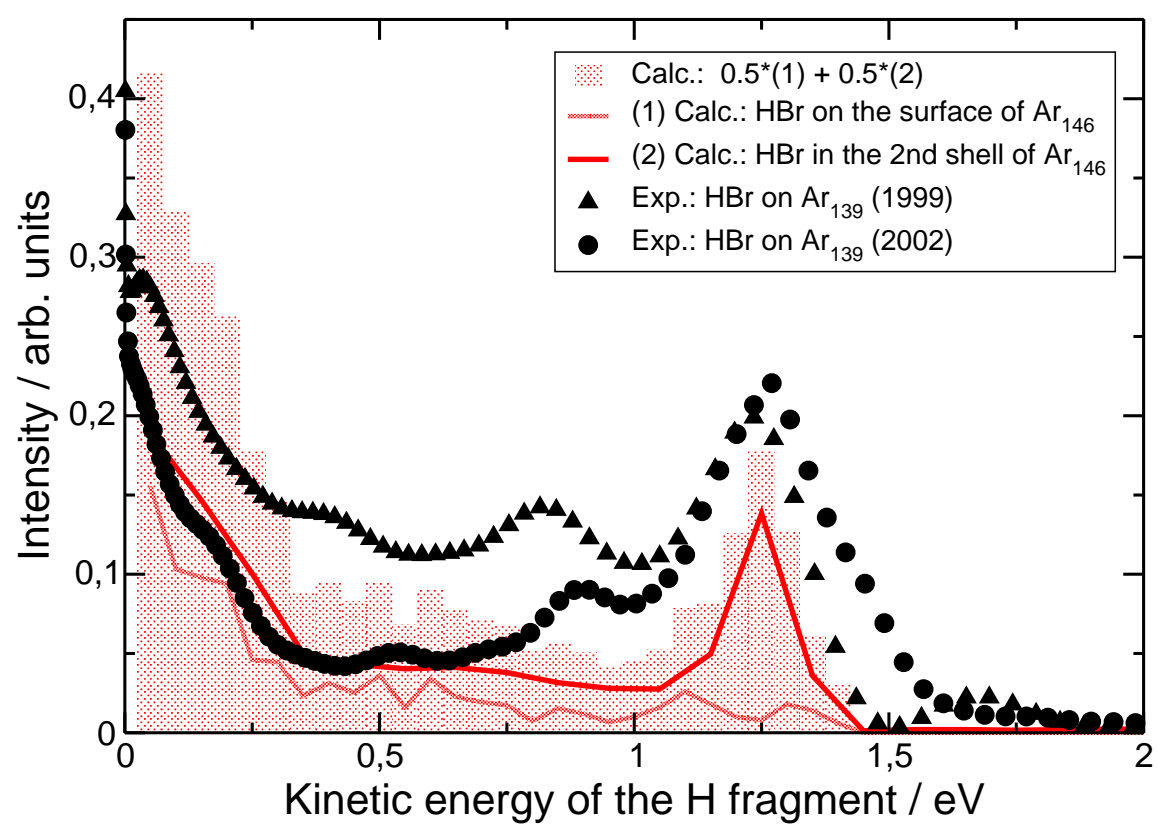

Abbildung 5.3: Die Abbildung zeigt Messungen und Rechnungen zur Photodissoziation von $\mathrm{HBr}$ auf $\mathrm{Ar}$ Clustern, $\langle n\rangle \approx 140$. Die Messung aus dem Jahr 1999 stammt aus [Bau00], die aus dem Jahre 2002 aus dieser Arbeit. Die Rechnungen gehen auf Petr Slavíček (2002) zurück [Sla02b]. HBr - $\mathrm{Ar}_{146}$ sollte nahezu eine Ikosaederstruktur mit drei Schalen und einem Ar Atom in der Mitte besitzen. Die Schalen werden von außen nach innen gezählt (Abb. 5.1 und Abb. 1.6). In Rechnung (1) wird als Anfangsposition des HBr Moleküls die äußere Schale des Ar Clusters, in (2) die zweite Schale des Clusters angenommen. Rechnungen von Holger Vach [Vac02] sagen voraus, daß etwa die Hälfte der $\mathrm{HBr}$ Moleküle in der Oberfläche und die Hälfte durch Solvatisierungseffekte in der zweiten Schale des Ar Clusters sitzen. Dies wird durch das Balkendiagramm in der Abbildung verdeutlicht, das sich zu je $50 \%$ aus Rechnung (1) und (2) zusammensetzt.

weiteren Energieübertrag korreliert. Für wärmere Ar Cluster kann dies bedeuten, daß sich aufgrund niedrigerer Bindungsenergie der $\mathrm{Ar}$ Atome die Eindringtiefe für $\mathrm{HBr}$ Moleküle erhöht. Die Adsorption des $\mathrm{HBr}$ Moleküls kann sogar ein lokales Schmelzen des Clusters verursachen. Dadurch dringt das Molekül in den Cluster ein, eventuell werden einige $\mathrm{Ar}$ Atome abgedampft und der Cluster rekristallisiert. In wärmeren Clustern erhöht sich die Mobilität der Atome, was einen direkten Käfigaustritt ebenfalls unwahrscheinlicher macht.

Experimentell wird eine Verstärkung des Käfigeffekts mit Erhöhung der Düsentemperatur beobachtet, die für ein tieferes Eindringen des $\mathrm{HBr}$ Moleküls in den Cluster spricht, 
teilweise bis in die dritte Schale. Der Vergleich mit den Messungen für die Photodissoziation von $\mathrm{HBr}$ im Zentrum des $\mathrm{Ar}$ Clusters zeigt, daß die Eindringtiefe nicht stark ansteigt.

\subsubsection{HI Molekül in der Ar Clusteroberfläche}

Der Vergleich zwischen Experiment und Theorie für die Photodissoziation von $\mathrm{HI}$ adsorbiert an Ar Clustern wird im Abschnitt 5.2 behandelt. Hier kann aufgrund der Anregungsund Kopplungsmechanismen, die in den Berechnungen nicht berücksichtigt werden, keine quantitative Übereinstimmung erzielt werden. Der sehr hohe Anteil von teilweise abgebremsten Fragmenten in den Experimenten bestätigt die Positionierung des HI Moleküls in der zweiten Schale des Clusters.

\subsection{3 $\mathrm{HBr}$ und $\mathrm{HCl}$ Moleküle in flüssigen Ne Clustern}

Auch zur Photodissoziation von $\mathrm{HBr}$ Molekülen in Ne Clusterumgebungen gibt es Rechnungen von P. Slavíček [Sla02a]. Als Anfangsposition wurde sowohl die Oberflächenposition als auch das Zentrum des Ne Clusters gewählt. Der Vergleich dieser Rechnungen mit der entsprechenden Messung in Abb. 5.4 zeigt sehr gute Übereinstimmung mit der Anfangsposition des Moleküls im Zentrum des Clusters.

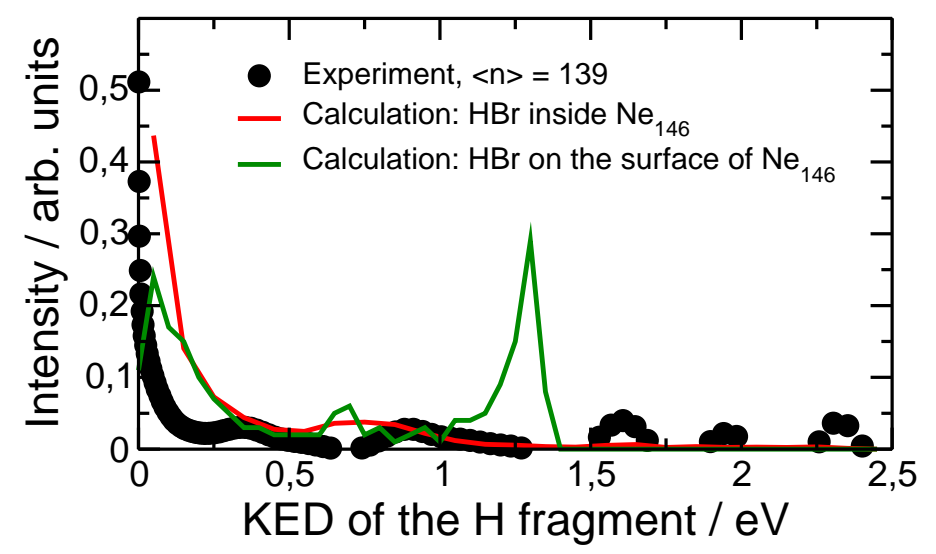

\footnotetext{
Abbildung 5.4: Vergleich zwischen Experiment und Theorie zur Photodissoziation von $\mathrm{HBr}$ Molekülen in Ne Clustern. Angeführt sind die theoretischen Berechnungen zur Photodissoziation von $\mathrm{HBr}$ Molekülen an der Oberfläche und im Zentrum von Ne Clustern [Sla02a]. Der Vergleich mit den experimentellen Ergebnissen zeigt, daß sich das $\mathrm{HBr}$ Molekül im Zentrum des Ne Clusters befindet. In der Clusterpräparation mittels Pick-up ist das nur bei flüssigen Clustern möglich.
} 
Die Berechnungen von $\mathrm{H}$. Vach zur Einlagerung von $\mathrm{HBr}$ Molekülen in die Oberfläche eines Ar Clusters zeigen, daß bei einem Cluster mit fester Schalenstruktur jede dieser Schalen eine Potentialbarriere für das eindringende Molekül darstellt. Aufgrund der zunehmenden Dichte zum Zentrum hin wird die Potentialbarriere in diese Richtung von Schale zu Schale höher. Berechnungen aus der Gruppe von R.B. Gerber haben gezeigt, daß es zwei Minima für den Aufenthaltsort des Moleküls in der Clusterumgebung gibt. Das ist zum einen die Oberflächenposition und zum anderen der Aufenthalt im Zentrum des Clusters. Energetisch sind die beiden Positionen nahezu identisch [Bau00]. Bei Clustern mit fester Strukur ist die Potentialbarriere der Schalen so hoch, daß es einem Molekül im Pick-up Prozeß nicht gelingt, diese Barriere zu überwinden. Es verbleibt in der Oberflächenumgebung.

Ein tieferes Eindringen des Moleküls in den Cluster in Folge des Pick-up Prozesses setzt einen Cluster in flüssiger Phase voraus. Beim Eindringen in den Cluster wird das Molekül zwar von den Ne Clusteratomen abgebremst, diese stellen aber aufgrund ihrer guten Mobilität nur eine geringe Potentialbarriere dar. Auch hier stellt die Position im Zentrum das absolute Minimum dar, wobei der Potentialtopf nicht so tief wie bei einem festen Cluster ist.

Für die größeren Cluster, für die hier Messungen durchgeführt worden sind (bis $\langle n\rangle=$ 1600), gibt es keine weiteren Berechnungen. Die Spektren zeigen bei einer Erhöhung der Clustergröße keine Veränderung. Das spricht dafür, daß auch die größeren Ne Cluster flüssig sind. Allerdings muß man berücksichtigen, daß schon bei Clustern der mittleren Größe von $\langle n\rangle=139$ die drei Schalen ausreichen, um so gut wie alle Wasserstofffragmente vollständig abzubremsen. Wenn man dieses Ergebnis auf die großen Cluster überträgt, bedeutet das, daß die $\mathrm{HBr}$ Moleküle bei diesen mindestens in die dritte Schale eindringen. Eine tiefere Position ( $\langle n\rangle=1600$ entspricht acht Ikosaederschalen) ist auch denkbar, läßt sich aber mit dieser Meßmethode nicht verifizieren.

Schon die Gruppe um T. Möller hat experimentell die flüssige Phase für kleine $\mathrm{Ne}$ Cluster bestätigt. Allerdings sehen sie im Clustergrößenbereich von $n=300$ einen Phasenübergang zu festen Strukturen [Pie97]. Ein Phasenübergang ist in diesen Experimenten nicht erkennbar. Es gibt keinen Hinweis dafür, daß große Ne Cluster fest sind. Für $\mathrm{HCl}$ zeigen die experimentellen Ergebnisse dasselbe Verhalten. Rechnungen zur Photodissoziation von $\mathrm{HCl}$ in großen $\mathrm{Ne}$ Clustern existieren nicht. 


\subsubsection{HBr Molekül im Zentrum des Ar Clusters}

Bei der Koexpansion von $\mathrm{HBr}$ mit Ar werden Ar Cluster mit einem $\mathrm{HBr}$ Molekül im Zentrum des Clusters gebildet. Die experimentellen Ergebnisse zeigen, daß der Käfigeffekt für größere Cluster immer mehr an Bedeutung gewinnt. Bei einer mittleren Clustergröße von $\langle n\rangle=$ 450 gelingt es den Wasserstofffragmenten nicht mehr, ungehindert den Cluster zu verlassen.

Die Erzeugung der Ar Cluster in einer Düsenstrahlexpansion führt zu Clustergrößen, die einer Log-Normalverteilung gehorchen. Interessant sind unter diesem Aspekt die Unter- und Obergrenze der Größenverteilung. Die volle Halbwertsbreite der Verteilung liefert jeweils für die obere und untere Grenze eine zugehörige Clustergröße. Cluster innerhalb dieses Größenbereichs liefern wesentliche Beiträge zu den Flugzeitspektren. Somit findet sich in Tab. 5.1 eine Gegenüberstellung zwischen der mittleren $\langle n\rangle$ und der kleinsten sowie größten nennenswerten Clustergröße $\left(n_{l}\right.$ und $\left.n_{u}\right)$ [Ste02].

Auch zu diesem Themenkomplex sind in der Gruppe von P. Jungwirth Energieverteilungen für die Wasserstofffragmente berechnet worden [Sla00]. Die Berechnungen sind in Abb. 5.5 dargestellt. Mit der Zunahme der Clustergröße ist eine Abnahme des freien Austritts aus dem Käfig verknüpft. Für Cluster mit mehr als 146 Ar Atomen, was drei vollen Ikosaederschalen entspricht, wird kein freier Austritt berechnet. Die Energieverteilung wird von vollständig abgebremsten Fragmenten beherrscht. Die beiden weiteren Rechnungen sind für zwei volle Ikosaederschalen $(n=54)$ und eine weitere halb gefüllte Schale ( $n=97$ ) durchgeführt worden.

Der Vergleich zwischen Experiment und Theorie hinsichtlich des Käfigeffekts ist in Tab. 5.1 dargelegt. Dabei geben $R_{\exp }$ und $R_{\text {calc }}$ das Verhältnis zwischen völlig abgebremsten und frei austretenden Wasserstofffragmenten im Experiment und den Rechnungen an.

Die kleinste gemessene Größe $(\langle n\rangle=51)$ läßt sich dabei quantitativ mit der zugehörigen berechneten Größe von $n=54$ vergleichen. Der experimentelle Wert für $R$ ist hier etwas größer als der aus der Rechnung. Dies läßt sich unter Berücksichtigung der Clusterschalen diskutieren. Eine Anzahl von 54 Atomen (plus ein Chromophor) ergibt bei Edelgasclustern den Abschluß der zweiten Ikosaederschale (s. Abschnitt 1.2.4). Bei der GröBernverteilung im Experiment im Bereich von $n_{l}=21$ bis $n_{u}=71$ besitzen die meisten Cluster eine zweischalige Struktur, die der in der Rechnung entspricht. Allerdings gibt es auch einige Cluster, bei denen schon die dritte Schale teilweise gefüllt ist $(54<n<71)$. Diese sind in der Lage, die Wasserstofffragmente besser abzubremsen, wodurch $R_{\exp }$ gegenüber der Rechnung erhöht wird. 


\begin{tabular}{|cccccc|}
\hline \multicolumn{3}{c}{ Experiment } & \multicolumn{3}{c|}{ Rechnungen } \\
$\langle n\rangle$ & $n_{l}$ & $n_{u}$ & $R_{\text {exp }}$ & $R_{\text {calc }}$ & $n$ \\
\hline \hline 51 & 21 & 71 & 1.3 & 0.8 & 54 \\
80 & 32 & 112 & 3.8 & & \\
139 & 56 & 195 & 5.8 & 7.0 & 97 \\
230 & 93 & 322 & 12.5 & & \\
290 & 117 & 406 & 15.4 & & \\
450 & 182 & 630 & 58.6 & $\infty$ & 146 \\
\hline
\end{tabular}

Tabelle 5.1: Die Ar Clustergröße wird unter Analyse der Log-Normalverteilung charakterisiert. $\langle n\rangle$ bezeichnet die mittlere Clustergröße der Messungen, $n_{l}$ die Clustergröße, die mit der unteren Grenze der vollen Halbwertsbreite der Verteilung korrliert ist, $n_{u}$ die obere. Der Vergleich zwischen Experiment und Theorie ist über die berechneten Clustergrößen $n$ verknüpft. Von Interesse sind die Verhältnisse zwischen perfect caging und direct exit $\left(R_{\exp }\right.$ und $\left.R_{\text {calc }}\right)$. Die experimentellen Daten für $\langle n\rangle=51$ und 80 stammen von Messungen aus [Bau99a].

Die Rechnung für $n=97$ beschreibt einen Cluster, dessen zweite Schale vollständig und dessen dritte Schale halb gefüllt ist. Der experimentelle Wert für $\langle n\rangle=80$ läßt sich in diesem Bereich einordnen. Obwohl sich die Messung mit einer mittleren Größe von $\langle n\rangle=139$ ziemlich nah am Abschluß der dritten Clusterschale befindet $(n=146)$ wird der Anteil von Clustern mit drei vollständigen Schalen nicht allzu groß sein. Dies ist auf eine Isomerenverteilung zurückzuführen, die der Größenverteilung überlagert ist. Cluster mit mehr als 147 Atomen haben unter Umständen keine drei vollständigen Schalen, weil die Füllung der vierten Schale schon vor Abschluß der dritten Schale beginnen kann [Har86]. Unter diesem Aspekt macht es Sinn, die Rechnung für eine Clustergröße von $n=97$ (zweieinhalb Schalen) ebenfalls für diesen Vergleich heranzuziehen. Dieser Wert für die Ausprägung des Käfigeffekts von $R_{\text {calc }}=7.0$ ist nur leicht gegenüber dem experimentellen Wert erhöht.

Im Bereich von zweieinhalb bis drei Clusterschalen liegen die Messungen mit $\langle n\rangle=$ $230, n_{l}=93$ und $\langle n\rangle=290, n_{l}=117$. Dies erklärt den höheren Anteil von perfect caging, als der in der Rechnung für $n=97$ ermittelte. Der in der Rechnung für drei und mehr Schalen vorausgesagte verhinderte Ausgang führt in den Messungen zu stark betontem Käfigeffekt.

Bei einer mittleren Clustergröße von $\langle n\rangle=450$ und damit einer unteren Grenze von $n_{l}=182$ besitzen alle Cluster mindestens drei Schalen. Im Mittel sind es drei bis 


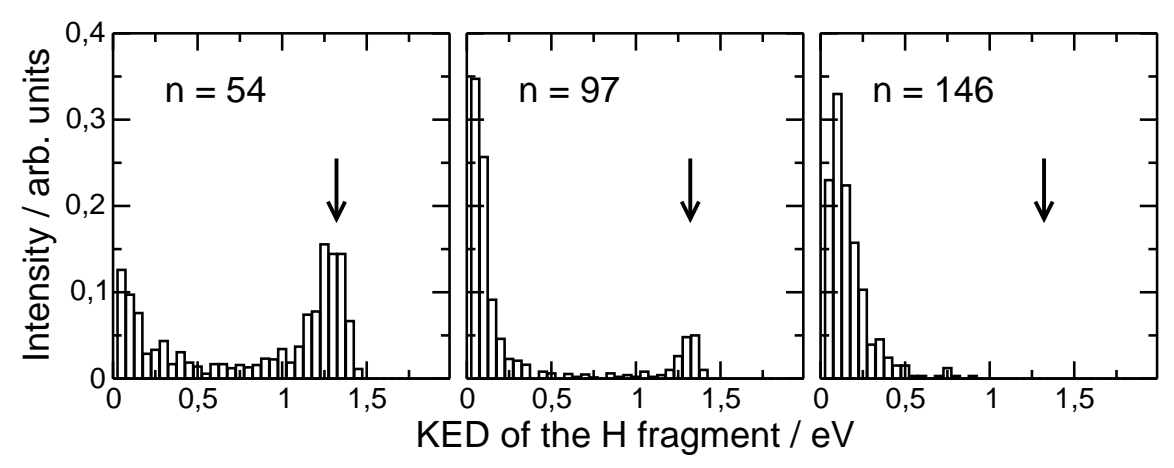

\begin{abstract}
Abbildung 5.5: Gezeigt sind theoretische Berechnungen zur Phtotodissoziation von $\mathrm{HBr}$ Molekülen, die sich im Zentrum von Ar Clustern befinden. Die Rechnungen stammen aus der Gruppe von P. Jungwirth [Sla00]. Die Ar Clustergröße $n$ ist in den Bildern vermerkt. Die Pfeile markieren die Energie für den freien Ausgang von Wasserstofffragmenten.
\end{abstract}

vier. Dabei sollte es vollständige Übereinstimmung zwischen dem experimentellen Ergebnis und der Rechnung für $n=146$ geben. Ein Vergleich des Käfigeffekts in diesem Fall erfordert eine tiefergehende Erklärung des Experiments. Das Verhältnis $R_{\text {exp }}=58.6$ bezieht sich auf den Energieraum. Die zugehörige Energieverteilung stammt aber aus der Transformation des gemessenen Flugzeitspektrums. Im Flugzeitraum beträgt das Verhältnis $R_{T O F}=108$. Wenn man weiterhin das Signal-zu-Rausch-Verhältnis von $R_{S / N}=30$ berücksichtigt erhält man folgende Fehlergrenzen im Flugzeitraum: Für die Flugzeit von $4.026 \mu \mathrm{s}\left(E_{\text {kin }}=0 \mathrm{eV}\right)$ beträgt die Intensität $I(4.026 \mu \mathrm{s})=271 \pm 9$. Für die Intensität bei $3.612 \mu \mathrm{s}\left(E_{k i n}=1.3 \mathrm{eV}\right)$ bedeutet dies unter Berücksichtigung des elektronischen Grundrauschens des digitalen Speicheroszilloskps $I(3.612 \mu \mathrm{s})=2.5 \pm 3$. Damit ergibt sich für das Käfigverhältnis im Flugzeitraum der mögliche Bereich $48<R_{T O F}<\infty$. Wenn man die im Vergleich zur Messung schlechte Statistik der Rechnung, die auf der geringen Anzahl der berechneten Trajektorien beruht mit einbezieht, könnte sich das Verhältnis $R_{\text {calc }}$ deutlich ändern. Schon ein einziges registriertes Ereignis bei der Energie des freien Austritts $\left(E=1.3 \mathrm{eV}\right.$ würde zu $R_{\text {calc }}=98$ führen. Im Bereich der Meß- und statistischen Fehler führen Experiment und Theorie hier zum gleichen Ergebnis.

Sowohl im Experiment als auch den Rechnungen kann in quantitativer Übereinstimmung gezeigt werden, daß Wasserstofffragmente aus der Photodissoziation von $\mathrm{HBr}$ den Ar Cluster nicht verlassen können, wenn er mehr als drei Schalen besitzt. 


\subsection{5 $\mathrm{HCl}$ Molekül in der Oberfläche von Edelgasclustern}

Die Photodissoziationsmessungen von $\mathrm{HCl}$ Molekülen adsorbiert auf $\mathrm{Ne}, \mathrm{Ar}, \mathrm{Kr}$ und $\mathrm{Xe}$ Clustern liefern folgende experimentelle Ergebnisse:

1. Direkter Käfigaustritt (direct exit) wird nicht beobachtet.

2. Alle Energieverteilungen werden von vollständig abgebremsten Wasserstofffragmenten dominiert (perfect caging).

3. Die gemessenen Wasserstofffragment-Energieverteilungen sind unabhängig von der Größe des Edelgasclusters.

4. Teilweise abgebremste Fragmente(delayed exit) werden nur bei der Adsorption auf großen Xe Clustern beobachtet.

Um diese Ergebnisse interpretieren zu können, muß zuerst die zentrale Frage dieses Abschnitts geklärt werden: Wo befindet sich das $\mathrm{HCl}$ Molekül in der Clusterumgebung?

Im Gegensatz zu den oben aufgeführten Rechnungen zu den Positionen von $\mathrm{HBr}$ und $\mathrm{HI}$ in $\mathrm{Ar}$ Clustern, existieren solche Rechnungen für $\mathrm{HCl}$ nicht. Wenn man die erwähnten und weitere Rechnungen von $\mathrm{H}$. Vach zum Moleküleinfang heranzieht [Vac00, Vac99] und die im Gegensatz zu $\mathrm{HBr}$ und $\mathrm{HI}$ schwächere Ar- $\mathrm{HCl}$ Wechselwirkung berücksichtigt, so ist davon auszugehen, daß sich das $\mathrm{HCl}$ Molekül direkt in der Oberfläche bzw. äußeren Schale des Clusters befindet. Auch eine Adsorption direkt auf der Oberfläche wäre denkbar.

In der Gruppe von R.B. Gerber sind Berechnungen zu Strukturen von HCI Molekülen auf Ar Clustern durchgeführt worden. Auch ein intensives Studium der Photodissoziationsdynamik im Käfig wurde betrieben. Aus diesen Studien stammen mit diesen Messungen qualitativ zu vergleichende Energieverteilungen der Wasserstofffragmente [Niv99, Niv97, Bau00]. Für diese Rechnungen wurden die $\mathrm{HCl}$ Moleküle an verschiedenen Positionen auf und in einer $\mathrm{Ar}_{54 / 55}$ Clusteroberfläche platziert. Die Strukturen und aus der Photodissoziation des $\mathrm{HCl}$ Moleküls stammenden Energieverteilungen sind in Abb. 5.6 dargestellt.

Die Berechnung der Energieverteilungen unterscheidet sich dahingehend von der $\mathrm{Me}-$ thode aus der Gruppe von P. Jungwirth, daß in der Dissoziation alle zwölf Potentiale berücksichtigt werden. Dieser quantenmechanischen Beschreibung des Dissoziationsprozesses steht eine semi-klassische Präparation des Anfangszustandes entgegen, die keine Librationen berücksichtigt. Für die $\mathrm{H}$-Ar Wechselwirkung wird anstelle der ab initio Po- 
tentiale ein Modell herangezogen. Die Kopplung aller Potentialflächen ermöglicht auch die Berechnung von Rekombinationen und Schwingungsanregungen [Bau00].
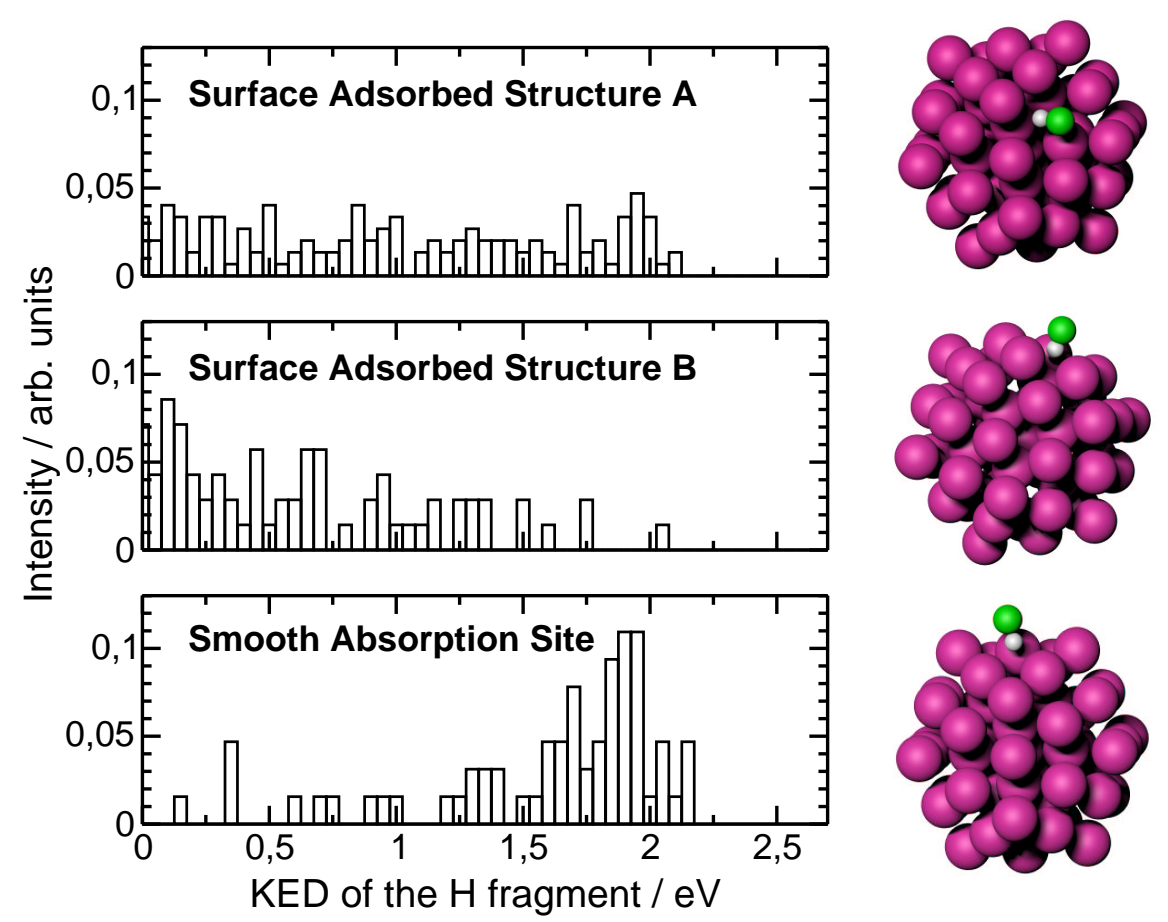

\begin{abstract}
Abbildung 5.6: Die möglichen Positionen und Orientierungen eines $\mathrm{HCl}$ Moleküls auf einem Ar Cluster und die Konsequenzen für die Energieverteilung der Wasserstofffragmente werden theoretisch behandelt. Sowohl die Strukturen als auch die berechneten Energieverteilungen aus der Photodissoziation von $\mathrm{HCl}$ auf $\mathrm{Ar}_{54 / 55}$ stammen aus der Gruppe von R.B. Gerber [Bau00].
\end{abstract}

Die drei Positionen, für die die Rechnungen voliegen sind reine Oberflächenpositionen. Die mit Smooth Absorption Site bezeichnete Konfiguration entspricht in der Beschreibung zu Anfang dieses Kapitels der Position (1). Das Molekül ist an der Oberfläche angelagert und dringt nicht in diese ein. Dies führt in diesen Berechnungen wie schon oben beschrieben zu deutlichem Käfigausgang, womit diese Konfiguration für die Experimente ausscheidet. Die beiden mit Surface Adsorbed Structure A/B gekennzeichneten Konfiguration entsprechen dabei Position (2). Sie unterscheiden sich dahingehend, daß bei Struktur A das Wasserstoffatom zwischen die erste und zweite Schale zeigt, währenddessen es bei Struktur B in Richtung Clusterzentrum zeigt. Insbesondere Struktur B, wo das Molekül in der Oberfläche 
steckt, schränkt durch die umgebenden Ar Atome sämtlichen Bewegungsfreiheitsgrade des Moleküls ein (constrained geometry). Dies führt dazu, das das Wasserstoffatom nach der Dissoziation den ganzen Cluster durchqueren muß, was eine starke bis vollständige $A b$ bremsung erzeugt. In Struktur A können die Wasserstofffragmente den Cluster seitlich mit geringer Wechselwirkung verlassen, was zu einer geringeren Ausprägung des Käfigeffekts führt.

Ein qualitativer Vergleich mit den Experimenten kann nur für Struktur B sprechen, bei der das $\mathrm{HCl}$ Molekül in der äußeren Schale des Ar Clusters steckt. Damit lassen sich die experimentellen Ergebnisse folgendermaßen erklären:

1. Bei dieser Konfiguration zeigen alle Wasserstofffragmente zum Zentrum des Clusters. Damit ist es ihnen unmöglich ohne Wechselwirkung mit den Clusteratomen den Käfig zu verlassen, was einem direkten Austritt entsprechen würde.

2. Die Orientierung der $\mathrm{HCl}$ Moleküle in der Clusteroberfläche bewirkt, daß die Wasserstofffragmente nach der Dissoziation den gesamten Cluster durchqueren müssen. Das bedeutet schon für die hier gemessenen Ar Cluster mit $\langle n\rangle=140$ Atomen und damit zwei bis drei Ikosaederschalen, daß das Wasserstofffragment mit linearer Trajektorie ungefähr fünf Schalen durchqueren muß. Wenn man dies mit der Photodissoziation von $\mathrm{HBr}$ Molekülen in Ar Clustern vergleicht, wo schon das Durchqueren von drei Schalen genügt, um die Wasserstofffragmente vollständig abzubremsen, so ist verständlich warum diese Verteilungen trotz höherer kinetischer Anfangsenergie $(2.0 \mathrm{eV}$ anstatt $1.3 \mathrm{eV})$ von perfect caging dominiert werden.

3. Die Größenunabhängigkeit geht aus der Erklärung zu Punkt 2 hervor. Wenn schon bei kleinen Clustern fünf Schalen zum vollständigen Abbremsen ausreichen, so verursacht ein Cluster mit $\langle n\rangle=1000$, bei dem von der Oberfläche ausgehend zwölf Schalen durchquert werden müssen, erst recht perfect caging.

4. Wenn man für die Photodissoziation von $\mathrm{HCl}$ in Xe Clustern Vergleiche mit früheren Messungen unserer Gruppe zu $\mathrm{HBr}$ und $\mathrm{HI}$ in dieser Umgebung anstellt [Bau01a], so sieht man, $\mathrm{da} B$ in $\mathrm{Xe}$ Clustern für $\mathrm{HBr}$ und $\mathrm{HI}$ der delayed exit die Verteilungen dominiert. Das ist auf das ungleiche Massenverhältnis zurückzuführen. Wenn man für die Abbremsung der Wasserstofffragmente im Cluster das einfachste Modell von zentralen Stößen mit den Clusteratomen annimmt, so veliert das Wasserstoffatom im Stoß mit einem Ne Atom $18 \%$ und mit Xe 3\% seiner kinetischen Energie. 
Damit ist es in Xe Clusterumgebungen deutlich schwieriger, leichte Fragmente wie Wasserstoffatome vollständig abzubremsen.

\title{
5.1.6 $\mathrm{HCl}$ Molekül in Ar und $\mathrm{HCl}$ Clustern
}

Die Photodissoziation kleiner $\mathrm{HCl}$ Cluster in großen Ar Clustern entspricht weitestgehend der von $\mathrm{HBr}$ Molekülen in Ar Clustern. Eine ausführliche Diskussion dazu findet sich in Abschnitt 5.1.4. Ein Unterschied kann sich in gegenseitigen Schwingungsanregungen der $\mathrm{HCl}$ Moleküle in der Clusterumgebung ergeben [Bau01c].

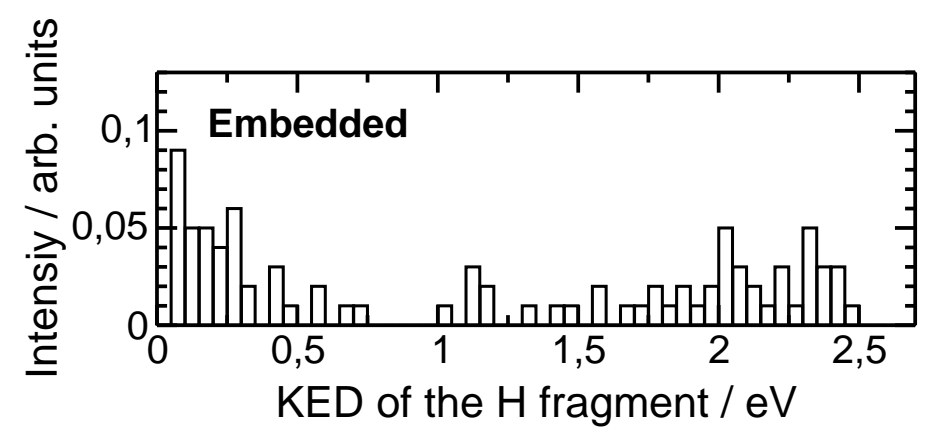

\begin{abstract}
Abbildung 5.7: Aus der Gruppe von R.B. Gerber stammt die berechnete Energieverteilung der Wasserstofffragmete aus der Photodissoziation von $\mathrm{HCl}$ eingebettet in einen $\mathrm{Ar}_{54}$ Cluster. Die angenommene Photonenenergie beträgt $7.0 \mathrm{eV}$, so daß ein quantitativer Vergleich mit dem Experiment (6.43 eV) nicht möglich ist [Niv97].
\end{abstract}

Dieses für kleine $\mathrm{HBr}$ Cluster in Ar Clustern beobachtete Phänomen, was sich in kinetischen Energien äußert, die größer als die des freien Austritts ist (Anregung), tritt für kleine $\mathrm{HCl}$ Cluster nicht auf. Ein weiterer Effekt, der sich in Energien jenseits der Anfangsenergie der freien Fragmente zeigt, die Rekombination, kann hier auch nicht beobachtet werden. Für $\mathrm{HBr}$ ist bei einer Wellenlänge von $243 \mathrm{~nm}$ die Wahrscheinlichkeit zur Dissoziation eines Moleküls in $v=1$ (erste Schwingungsanregung) gegenüber der für $v=0$ um einen Faktor zehn überhöht [Pou98, Bau99b]. Schon bei einer Dissoziationswellenlänge von $193 \mathrm{~nm}$ konnte dies bei $\mathrm{HBr}$ nicht mehr beobachtet werden $(v=1$ sieben mal kleiner als $v=0$ ) [Nah98, Bau99b]. Für schwingungsangeregtes $\mathrm{HCl}$ ist der Dissoziationsquerschnitt um einen Faktor zwei kleiner als für $\mathrm{HCl}$ im Grundzustand [Reg00, Che02], so daß sich eine Schwingungsanregung hier nicht nachweisen läßt.

Der wesentliche Unterschied zwischen $\mathrm{HBr}$ und $\mathrm{HCl}$ liegt in der kinetischen Anfangs- 
energie der Wasserstofffragmente. Bei der Photodissoziation von $\mathrm{HBr}$ beträgt sie $1.3 \mathrm{eV}$ (Laser: $243 \mathrm{~nm}$ ) und bei $\mathrm{HCl} 2.0 \mathrm{eV}$ (Laser: $193 \mathrm{~nm}$ ). Dies in Kombination mit den im Vergleich zu $\mathrm{HBr}$ kleineren mittleren Ar Clustergrößen verursacht den höheren Anteil an direct exit.

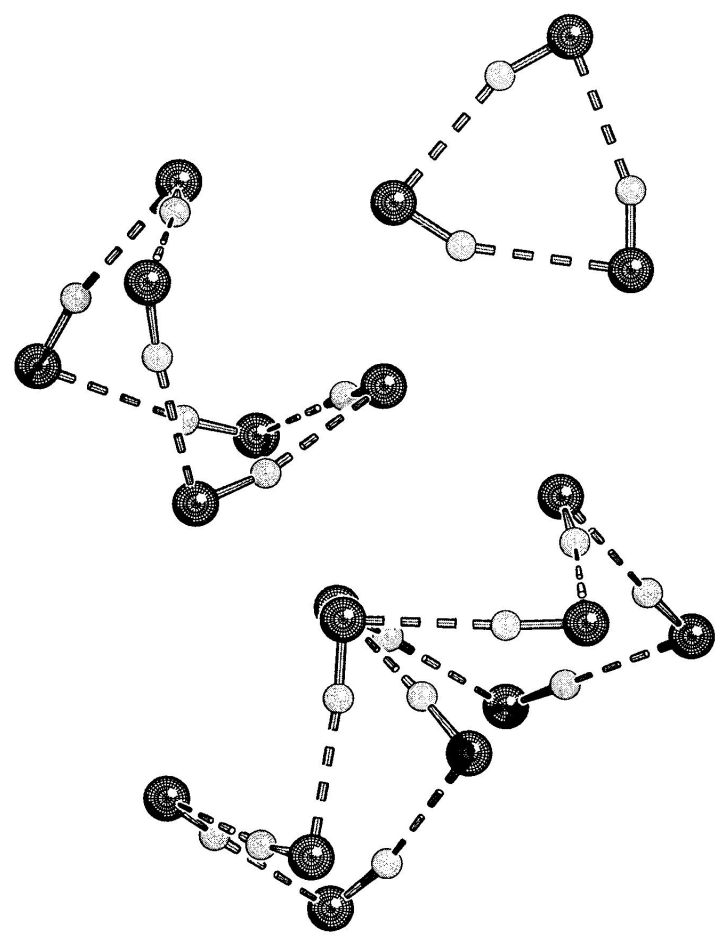

Abbildung 5.8: Minimumskonfiguration von $\mathrm{HCl}_{\mathrm{n}}, \mathrm{n}=3,6$ und 10 von J.G. Siebers [Sie97] berechnet mit einem intermolekularen $\mathrm{HCl}-\mathrm{HCl}$ Potential [Vot83].

Auch hier gibt es wie für die Adsorption von $\mathrm{HCl}$ auf der Oberfläche von $\mathrm{Ar}$ Clustern eine berechnete Energieverteilung aus der Gruppe von R.B. Gerber, die in Abb. 5.7 dargestellt ist. Der qualitative Vergleich zeigt gute Übereinstimmung mit den Messungen. In beiden Fällen werden die Verteilungen von direct exit und perfect caging beherrscht. Teilweise abgebremste Fragment (delayed exit) spielen nur eine untergeordnete Rolle.

Die Photodissoziation von $\mathrm{HCl}$ Molekülen in kleinen $\mathrm{HCl}$ Clustern $(\langle n\rangle=6)$ ist mit früheren Messungen unserer Gruppe zur Photodissoziation von kleinen $\mathrm{HBr}$ Clustern $(\langle n\rangle=8)$ bei einer Wellenlänge von $193 \mathrm{~nm}$ vergleichbar [Nah98, Bau99b]. Kleine $\mathrm{HCl}$ und $\mathrm{HBr}$ Cluster besitzen eine relativ offene Struktur die zu einer hohen Wahrscheinlich- 
keit für direkten Austritt oder Austritt nach einem Stoß führt. Die Strukturen für $(\mathrm{HCl})_{n}$ $(n=3,610)$ sind in Abb. 5.8 dargestellt [Vot83, Sie97].

\subsubsection{Zusammenfassung}

Abschließend zeigt die Photodissoziation von $\mathrm{HCl}, \mathrm{HBr}$ und $\mathrm{HI}$ Molekülen in Edelgasclusteroberflächen, daß die Eindringtiefe in den Cluster von $\mathrm{HI}$ zu $\mathrm{HCl}$ abnimmt und in gleicher Richtung der Anteil vollständig abgebremster Wasserstofffragmente ansteigt. Letzteres ist auf die starrere Geometrie des Moleküls in der Oberfläche zurückzuführen. Auf Energieverteilungen, die aus der Dissoziation von Oberflächenmolekülen stammen, hat die Clustergröße keinen Einfluß. Aufgrund der Masse der Edelgasatome nimmt der Anteil teilweise abgebremster Fragmente in Richtung der Xe Cluster zu. Hier zeigen auch Experimente und zugehörige Rechnungen zur Photodissoziation von $\mathrm{HCl}$ und $\mathrm{HBr}$ in Edelgasmatrizen ( $\mathrm{Ar}$, $\mathrm{Kr}, \mathrm{Xe})$, denen Betrachtungen der Mobilität $(10-40 \AA)$ und Thermalisierungszeit (0.4$1.1 \mathrm{ps)}$ von Wasserstofffragmenten zu Grunde liegen, daß der Transfer der kinetischen Energie mit steigender Masse des Edelgasatoms abnimmt [Elo99, Vas99].

Für die Photodissoziation eines Halogenwasserstoffmoleküls im Inneren eines Edelgasclusters ist eine deutliche Clustergrößenabhängigkeit festzustellen. Mit Erhöhung der Clustergröße und damit der Schalenanzahl nimmt der direct exit zu Gunsten des perfect caging bis auf Null ab.

Eine Überblick über frühere Messungen unserer Gruppe auf diesem Gebiet liefert der Übersichtsartikel von U. Buck [Buc02].

\subsection{Winkelverteilung in den Produktkanälen}

Im letzten Abschnitt stand als asymptotische Eigenschaft der Photodissoziation die Energieverteilung der Fragmente im Mittelpunkt der Betrachtungen. Hier soll nun die Bevölkerung der Produktkanäle unter Einfluß der Clusterumgebung diskutiert werden. Zum Vergleich werden dazu eigene Messungen sowie Rechnungen und Messungen zur Photodissoziation der freien Moleküle herangezogen.

\subsubsection{HI: Laserpolarisation $\alpha$}

Für die Photodissoziation von freien HI Molekülen mit einer Wellenlänge von $243 \mathrm{~nm}$ gibt es für die Anregung und die resultierenden Produktkanäle experimentell und theoretisch 
eindeutig bewiesene Eigenschaften [Roy02]. An der Photodissoziation sind mehrere Übergänge beteiligt, es gibt aber keine Kopplungen, die zu Umverteilungen in den Produktkanälen führen. Der zu etwa $50 \%$ an der Dissoziation beteiligte parallele Übergang in den ${ }^{3} \Pi_{0}^{+}$ Zustand führt in den Kanal I $\left(\beta^{*}=2.00\right)$, währenddessen die senkrechten Übergänge ${ }^{1} \Pi_{1}$ und ${ }^{3} \Pi_{1}$ zu je $25 \%$ den Grundzustand bevölkern $(\beta=-1.00)$. Die Besetzung der beiden Produktkanäle ist nahezu gleichverteilt $(R=0.93)$.
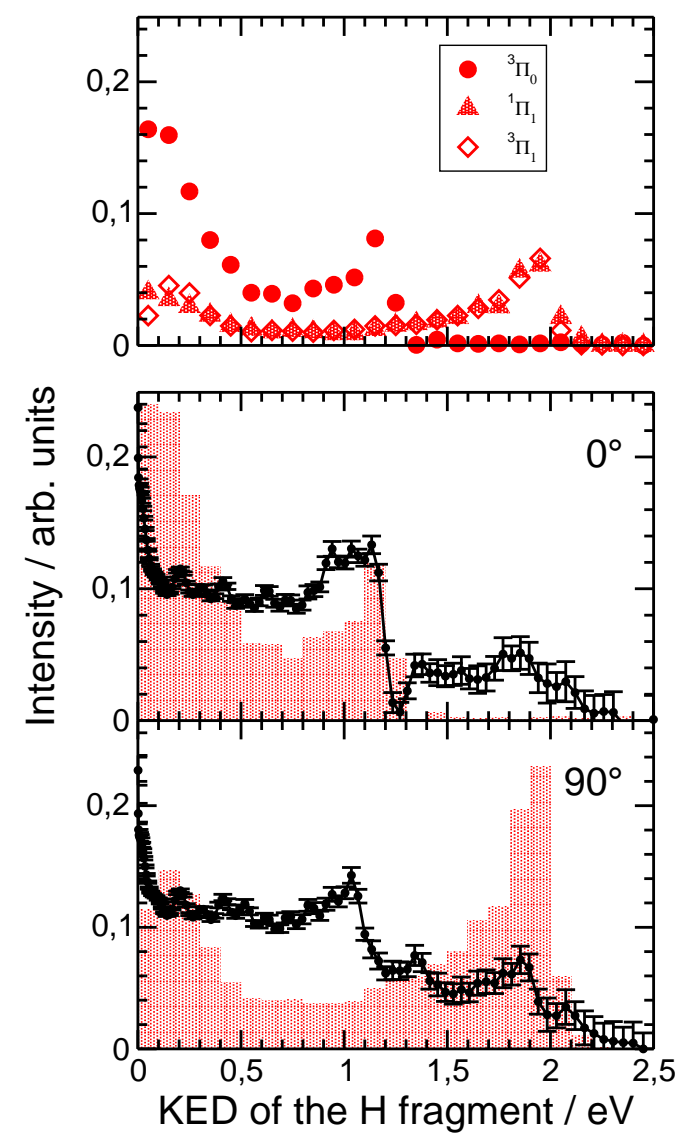

Abbildung 5.9: Der Vergleich zwischen Berechnungen zur Photodissoziation von $\mathrm{HI}$ Molekülen auf Ar Clustern [Sla02b] mit den hier vorliegenden Experimenten zeigt deutliche Abweichungen. Die Berechnungen liegen für die drei Übergänge aus dem Grundzustand $X^{1} \Sigma_{0}^{+}$in ${ }^{1} \Pi_{1},{ }^{3} \Pi_{1}$ und ${ }^{3} \Pi_{0}^{+}$vor. Dabei werden keine Kopplungen in der Anregung oder der Dissoziation berücksichtigt.

Die Situation bei der Photodissoziation von $\mathrm{HI}$ in einer Ar Clusteroberfläche stellt sich 
im Vergleich zum freien Molekül vollständig anders dar. Das Interesse gilt den Wasserstofffragmenten, die im direkten Austritt den Cluster verlassen. Energetisch weisen sie das gleiche Verhalten wie die Fragmente aus der Dissoziation der freien Moleküle auf. Unterschiede ergeben sich in der Winkelverteilung der Fragmente, die im folgenden in Abhägigkeit von der Clusterumgebung diskutiert werden.

P. Slavíček hat für die drei oben genannten Übergänge die Photodissoziation von $\mathrm{HI}$ Molekülen in der zweiten Schale eines $\operatorname{Ar}_{146}$ Clusters berechnet. Die daraus resultierenden Energieverteilungen der Wasserstofffragmente sind gewichtet mit dem Absorptionsquerschnitt der Photodissoziation (s. Abb. 1.4) im oberen Bild von Abb. 5.9 dargestellt.

Ein Vergleich mit der Messung zur Photodissoziation von $\mathrm{HI}$ in einer $\operatorname{Ar}_{139}$ Clusteroberfläche mit Laserpolarisationen von $0^{\circ}$ und $90^{\circ}$ ist im unteren Teil der Abbildung zu sehen. Dabei wird die Rechnung zum parallelen Übergang in den ${ }^{3} \Pi_{0}^{+}$Zustand mit der Messung unter einer Laserpolarisation von $0^{\circ}$ verglichen. Gleiches gilt für die Zustände $X^{1} \Sigma_{0}^{+}$und ${ }^{1} \Pi_{1},{ }^{3} \Pi_{1}$ (Gewichtung 1:1) und die Laserpolarisation senkrecht zur Detektorachse $\left(90^{\circ}\right)$. Die Skalierung bezüglich der Intensitäten bezieht sich auf die vollständig und teilweise abgebremsten Fragmente.

Wie läßt sich der deutliche qualitative Unterschied zwischen Experiment und Rechnung erklären? Im folgenden soll diese Frage unter Berücksichtigung unseres Meßprinzips und der Methode der Rechnungen beantwortet werden.

In diesem Zusammenhang ist eine prinzipielle Eigenschaft der Dissoziation in Lösungen zu beachten. Den ungehinderten Austritt (direct exit) aus dem Cluster gibt es in dem Sinne nicht. Hier muß zwischen den beiden Meßgrößen Winkelverteilung und Energieverteilung unterschieden werden. Die kinetische Energie eines Fragments wird durch einen „streifenden“ Stoß oder durch Streuung kaum verändert, so daß eine einzige derartige Wechselwirkung mit einem Käfigtom immer noch als direct exit bezeichnet werden kann. Für die Winkelverteilung der Fragmente sieht das völlig anders aus. Eine einzige Streuung an einem Käfigatom führt zwar zu keinem Energieverlust, kann aber je nach Stoßparameter die Richtung der Fragmenttrajektorie völlig ändern. Wie sich dies auf die Flugzeitspektren aus unseren Messungen auswirkt ist in Abb. 5.10 illustriert.

Hier ist nur der Fall der Laserpolarisation senkrecht zur Detektorachse dargestellt. Das Prinzip läßt sich auf parallele Ausrichtung übertragen. Der Laser dissoziiert sowohl Moleküle mit senkrechtem Übergangsdipolmoment (rotes Molekül) als auch mit einem Übergangsdipolmoment parallel zur Molekülachse (schwarzes Molekül). Um das Beispiel mit der Photodissoziation von $\mathrm{HI}$ in der Oberfläche von Ar Clustern vergleichen zu können, 


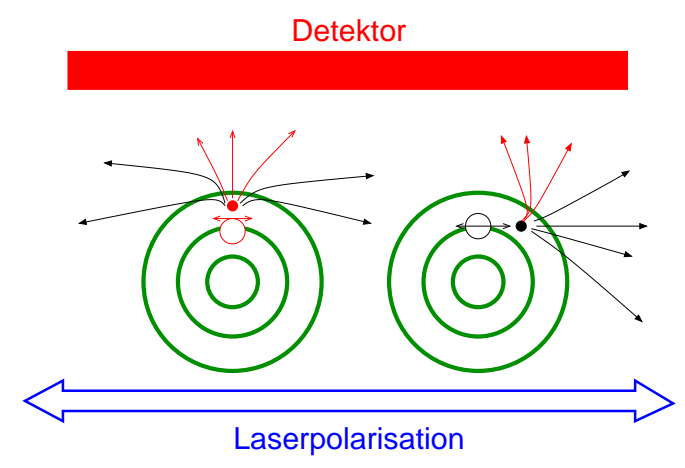

Abbildung 5.10: Einfluß der Clusterumgebung auf die Winkelverteilung der Fragmente. Dieses Bild erklärt die Photodissoziation eines Moleküls im Cluster analog zur Photodissoziation eines freien Moleküls in Abb. 3.2. Die Clusterschalen sind grün gekennzeichnet. Die Fragmenttrajektorien werden im Cluster durch Stöße und Streuung in ihrer Richtung verändert. Nur die mit roten Pfeilen markierten Trajektorien werden detektiert. Insgesamt werden die Winkelverteilungen isotroper.

sind die Moleküle hier auch in die zweite Clusterschale eingebaut (grüne Ringe). Bei der Dissoziation eines freien Moleküls entfernt sich das Wasserstofffragment auf einer Flugbahn parallel zur Molekülachse vom Dissoziationsort. Dies würde in dieser Illustration bedeuten, daß nur das Wasserstofffragment aus dem roten Molekül den Detektor erreichen könnte. Die Clusterumgebung führt hier jedoch zu einer dramatischen Änderung. Die Ar Atome der äußeren Schale lenken die Wasserstofffragmente durch Streuung und Stöße derart ab, daß eine deutliche Richtungsänderungen der Trajektorien auftritt. Dies führt dazu, daß Fragmente aus der Dissoziation des roten Moleküls vom Detektor weggestreut werden und Fragemente aus dem schwarzen Molekül auf diesen umgelenkt werden. Die Fragmente verlieren die Information über ihre ursprüngliche Flugrichtung und die Winkelverteilung wird isotroper.

Für die Photodissoziation von $\mathrm{HI}$ in der zweiten Schale eines $\operatorname{Ar}_{139}$ Clusters hat das folgende Konsequenzen: Im Laserpuls werden immer Moleküle über die senkrechten $\left(X^{1} \Sigma_{0}^{+}\right.$ und ${ }^{1} \Pi_{1},{ }^{3} \Pi_{1}$ in den Grundzustand) und den parallelen Übergang $\left({ }^{3} \Pi_{0}^{+}\right.$in den angeregten Zustand) dissoziiert. Streuung und Stöße in der Clusterumgebung führen zu nahezu isotropen Winkelverteilungen. Dies bedeutet, daß man sowohl unter einer Laserpolarisation von $0^{\circ}$ als auch von $90^{\circ}$ beide Produktkanäle nahezu gleichverteilt beobachtet.

Die qualitativ schlechte Übereinstimmung zwischen Experiment und Rechnungen ist auf unterschiedliche Detektionsverfahren zurückzuführen. In unseren Experimenten werden die 
Wasserstofffragmente an einem nahezu punktförmigen Detektor nachgewiesen, währenddessen in den MD Simulationen alle Fragmente, die den Cluster verlassen, unabhängig von der Richtung ihrer Trajektorien nachgewiesen werden. Die Rechnungen besitzen einen Kugeldetektor, der den Cluster umgibt, das Experiment betrachtet einen sehr schmalen Kegelförmigen Ausschnitt dieser Kugel in einer Richtung. Die Anistotropie in der Rechnung wird nicht über die Detektion der Fragmente sondern über die Präparation der Moleküle in unterschiedliche angeregte Zustände erreicht. Für einen aussagefähigen Vergleich zwischen Experiment und Rechnung müßten in den MD Simulationen die Winkelverteilungen der aus dem Cluster austretenden Fragmente ermittelt werden. Andere Rechnungen aus der Gruppe von R.B. Gerber bestätigen, daß die Dissoziation eines Fragments, das von einer Clusterschale umgeben ist, deutlich isotroper abläuft als die des freien Moleküls (s. Abb. 5.11 für $\mathrm{HCl}$ in $\mathrm{Ar}_{12}$ im Vergleich zu freiem $\mathrm{HCl}$ ).

Zwei weitere Möglichkeiten zu Umverteilungen in den Produktkanälen, die mit unserer Apparatur experimentell nicht studiert werden können, sollen im folgenden kurz erläutert werden.

Die Clusterumgebung verändert das Übergangsdipolmoment $\vec{\mu}$ und verursacht damit eine Kopplung in der Anregung. Eine clusterinduzierte Kopplung hängt von der Stärke der Rg-HX Wechselwirkung ab und müßte sich deswegen mit der Wahl des Edelgases ändern.

Eine Kreuzung der auseinanderlaufenden Potentiale führt zu einer Mischung der Produktkanäle. Es müßte für $\mathrm{HI}$ eine clusterinduzierte Kreuzung zwischen den ${ }^{1,3} \Pi_{1}$ und ${ }^{3} \Pi_{0}^{+}$ entstehen, die zu einer Umverteilung mit der Wahrscheinlichkeit $\mathrm{p}$ führt:

$$
{ }^{1,3} \Pi_{1} \underset{(1-p)}{\stackrel{p}{\rightleftarrows}}{ }^{3} \Pi_{0}^{+}
$$

Diese Umverteilung in beide Richtungen kann ein Erklärungsansatz für die fast vollständige Isotropie der beiden Kanäle sein.

\subsection{2 $\mathrm{HCl}$ : Laserpolarisation $\alpha$}

Bei der Photodissoziation von $\mathrm{HCl}$ eingebettet in einen $\mathrm{Ar}_{100}$ Cluster müssen die Wasserstofffragmente zwei bis drei Schalen durchqueren. Die experimentellen Ergebnisse (s. Abschnitt 4.3.3) zeigen hier nur vollständig abgebremste Fragmente. Vollständige Abbremsung bedeutet mindestens 15 zentrale Stöße Energieverlunst pro Stoß: $10 \%$ ), wobei jeder Stoß eine Änderung der Flugrichtung verursacht. Dies führt zu vollständigem Verlust der 
Information über die Anfangsflugrichtung der Wasserstofffragmente. Die Winkelverteilung der abgebremsten Fragmente ist völlig isotrop.

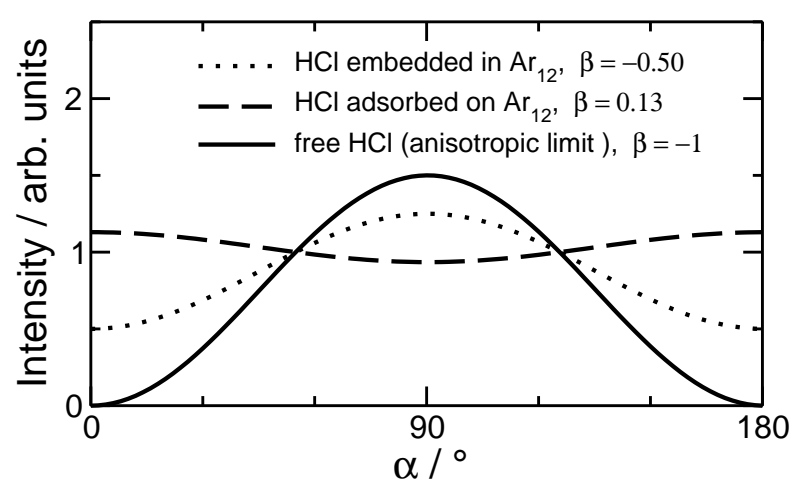

Abbildung 5.11: Winkelverteilung der Wasserstofffragmente aus der Photodissoziation von $\mathrm{HCl}-\mathrm{Ar}_{12}$. Der Winkel $\alpha$ gibt den Winkel der Laserpolarisation zur Detektorachse an und entspricht damit der Projektion des Übergansdipolmoments $\vec{\mu}$ auf die Detektroachse. Die Berechnung stammt aus der Gruppe von R.B. Gerber [Niv99].

In der Gruppe von R.B. Gerber ist dieses Verhalten durch Rechnungen bestätigt worden [Niv99]. In Abb. 5.11 sind zum Vergleich die Winkelverteilungen für die Dissoziation von freien $\mathrm{HCl}$ Molekülen, $\mathrm{HCl}$ in einem $\mathrm{Ar}_{12}$ Cluster und auf der Oberfläche eines solchen Clusters dargestellt. Aus der Innenposition heraus müssen die Wasserstofffragmente eine Clusterschale durchqueren. Dies führt zu einer Veränderung des Anisotropieparameters von $\beta=-1$ zu $\beta=-0.5$. Wenn sich das Molekül auf der Clusteroberfläche befindet (Wasserstoffatom zeigt nach innen) muß das Wasserstofffragment den ganzen Cluster durchqueren, was zwei Schalen entspricht. Hier beträgt der Anisotropieparameters $\beta=$ 0.13 , was nahezu einer isotropen Verteilung entspricht. Diese Rechnung ist direkt mit unseren Experimenten vergleichbar, weil auch hier das Wasserstofffragment - zwar aus dem inneren des Clusters - zwei Schalen durchqueren muß.

\subsubsection{Zusammenfassung}

Die Photodissoziation eines Moleküls in einer Clusterumgebung führt durch Mechanismen des Käfigeffekts immer zu einer Beeinflussung der Winkelverteilung der Fragmente. Wenn die Fragmente nur eine Schale durchqueren müssen, wird die Winkelverteilung durch Streuung und Stöße isotroper. In einem Cluster mit zwei oder mehr Schalen ist die Anzahl der 
Stöße so hoch, daß die Information über die Anfangsflugrichtung der Fragmente vollständig verlorengeht. Die Winkelverteilung ist isotrop.

\subsection{Dissoziation von $\mathrm{HXel}, \mathrm{HXeBr}$ und $\mathrm{HXeCl}$}

In disem Abschnitt soll geklärt werden, warum wir die Asymmetrie in den Flugzeitspektren aus der Photodissoziation von $\mathrm{HI}$ und $\mathrm{HCl}$ in Xe Clusteroberflächen der Existenz von $\mathrm{HXel}$ und $\mathrm{HXeCl}$ zuschreiben.

\subsubsection{Bildung}

Die Moleküle $\mathrm{HXel}$ und $\mathrm{HXeCl}$ können unter den hier gegebenen experimentellen $\mathrm{Be}$ dingungen gebildet werden. Das ist sowohl durch eine Vielzahl von Matrixexperimenten [Pet95, Pet99, Khr00, Pet97] als auch für Rechnungen in Clustern [Coh01] gezeigt worden. Der Bildungsprozeß ist in Abschnitt 1.4.1 erklärt. Nach der Photodissoziation von $H X$ verläßt das Wasserstofffragment die nächstgelegene Käfigumgebung, wird von einem Edelgasatom zurückgestoßen und bildet mit dem zurückgebliebenenm Molekül $\mathrm{X}^{-} \mathrm{Rg}^{+}$das Molekül HRgX.

\begin{tabular}{|lr|}
\hline Molekül & $\boldsymbol{D}_{\mathbf{0}}[\mathbf{e V}]$ \\
\hline \hline $\mathrm{HArF}$ & $0.35^{1}$ \\
$\mathrm{HKrF}$ & $0.7^{2}$ \\
$\mathrm{HKrCl}$ & $0.4^{2}$ \\
$\mathrm{HXeCl}$ & $0.9^{3}$ \\
$\mathrm{HXeBr}$ & $0.6^{2}$ \\
$\mathrm{HXel}$ & $0.42^{4}$ \\
\hline
\end{tabular}

Tabelle 5.2: Dissoziationsenergien von HRgX Molekülen. Referenzen: ${ }^{1}[\mathrm{Khr00}],{ }^{2}$ [Pet02a], ${ }^{3}[\mathrm{Joh} 99]$, ${ }^{4}[$ Pet99].

Die aus Matrixexperimenten bekannten Dissoziationsenergien der bisher beobachteten HRgX Spezies sind in Tab. 5.2 aufgeführt. Die Stabilität der Moleküle wächst mit kleiner werdender Masse des Halogenatoms. Für die leichteren Systeme dominiert die stärkere Coulomb Wechselwirkung die Bindung. 
Prinzipiell sollte die Bildung all dieser Spezies mit unserer experimentellen Methode möglich sein, wobei Photodissoziationsexperimente mit HF nicht durchgeführt wurden. Zur Nachweismöglichkeit der verbleibenden Spezies wird weiter unten Stellung genommen.

$\mathrm{Zu}$ einer erhöhten Bildungsrate von $\mathrm{HRgX}$ kann es insbesondere in Xe Clustern durch eine höhere Konzentration von Wasserstoffatomen kommen. Diese kann aus der Photodissoziation von adsorbierten Kohlenwasserstoffen stammen, wobei die großen Xe Cluster einen besonders großen Einfangquerschnitt für Kohlenwasserstoffmoleküle aus dem Untergrund haben. Untersuchungen hierzu ließen sich anstellen, wenn man $\mathrm{HI} / \mathrm{HCl}$ durch die deuterierten Moleküle ersetzen würde.

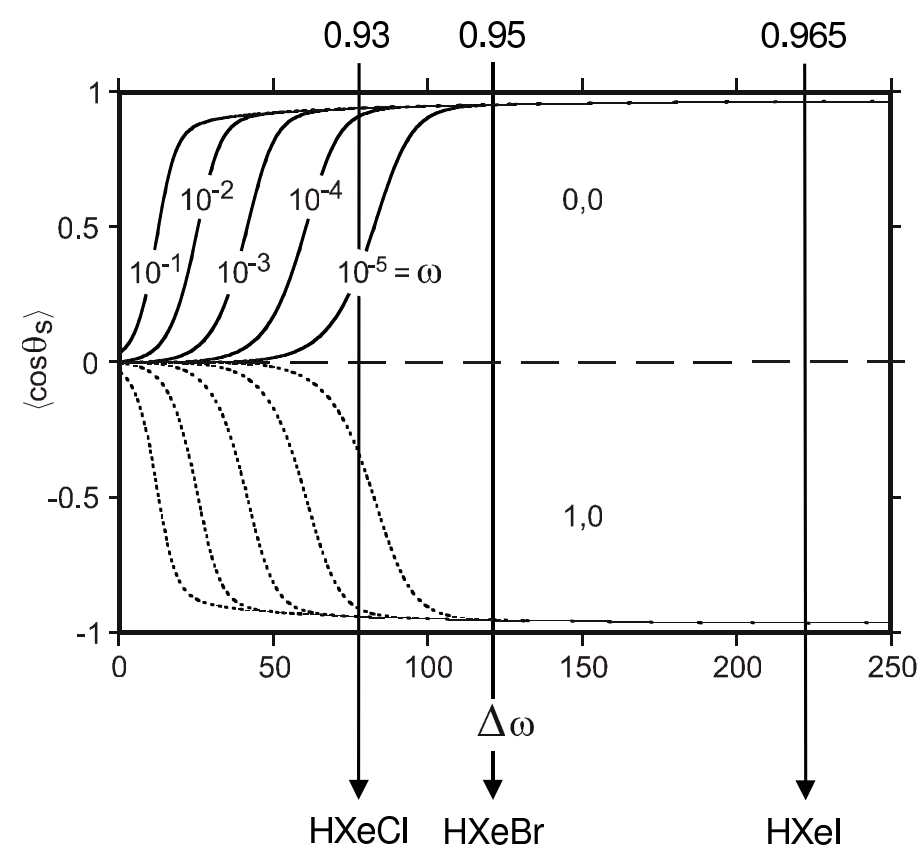

Abbildung 5.12: Nach Berechnungen von B. Friedrich kann der Oientierungskosinus $\left\langle\cos \theta_{S}\right\rangle$, der den Orientierungsgrad spezifiziert, für die Experimente dieser Arbeit angegeben werden [Nah02]. Mit $\left\langle\cos \theta_{S}\right\rangle>0.8$ kann man von einer Orientierung sprechen. Mit $\omega \approx 10^{-2}$ ist dies für $\Delta \omega>30$ erreichbar. Die hier verwendeten Moleküle sind mit den Werten für $\left\langle\cos \theta_{S}\right\rangle$ in der Abbildung vermerkt. Im oberen Teil ist der Orientierungsgrad für den Grundzustand $|\tilde{J} M\rangle=0,0$ gezeigt, im unteren für den ersten Pendelzustand $|\tilde{J} M\rangle=1,0$.

Ein Molekül, das bei der Anwesenheit von mehreren Wasserstoffatomen in einer Edelgasumgebung immer gebildet wird ist $\mathrm{HXeH}$ [Pet99]. Unter diesen Bedingungen ist davon 


\begin{tabular}{|lcccccccc|}
\hline Molekül & $\begin{array}{c}\Delta \alpha \\
{\left[\AA^{3}\right]}\end{array}$ & $\begin{array}{c}B \\
{\left[\mathrm{~cm}^{-1}\right]}\end{array}$ & $\begin{array}{c}\mu \\
{[\mathrm{D}]}\end{array}$ & $\begin{array}{c}E_{S} \\
{[\mathrm{kV} / \mathrm{cm}]}\end{array}$ & $\begin{array}{c}I_{L} \\
{\left[\mathrm{~W} / \mathrm{cm}^{2}\right]}\end{array}$ & $\Delta \omega$ & $\left\langle\cos \theta_{S}\right\rangle$ \\
\hline \hline $\mathbf{H X e l}$ & 5.5 & 0.0268 & 6.4 & 0.0042 & 0.017 & $1.1 \cdot 10^{11}$ & 225 & 0.965 \\
$\mathbf{H X e B r}$ & 4.5 & 0.0420 & 7.3 & 0.0042 & 0.012 & $1.1 \cdot 10^{11}$ & 117 & 0.95 \\
$\mathbf{H X e C l}$ & 3.9 & 0.0843 & 7.2 & 0.0078 & 0.011 & $1.7 \cdot 10^{11}$ & 80 & 0.93 \\
\hline $\mathbf{H I}$ & 0.43 & 6.551 & 0.44 & 0.0042 & 0.005 & $1.1 \cdot 10^{11}$ & 0.072 & 0.002 \\
\hline
\end{tabular}

Tabelle 5.3: Orientierungsgrad $\left\langle\cos \theta_{S}\right\rangle$ der Moleküle $\mathrm{HXel}, \mathrm{HXeBr}, \mathrm{HXeCl}$ und $\mathrm{HI}$ nach den experimentellen Parametern aus Abschnitt 4.4, den Mölekülkonstanten aus Tab. 1.4, mit den Formeln aus GI. 1.47 und den Rechnungen von B. Friedrich (s. Abb. 5.12).

auszugehen, daß es auch in unseren Clusterexperimenten gebildet wird. Allerdings handelt es sich hier im Gegensatz zu HRgX um ein spiegelsymmetrisches Molekül. Es besitzt keine ausgezeichnete Richtung und kann damit nicht orientiert werden. Dies führt in den Flugzeitspektren zu einer symmetrischen Verteilung, die sich aus dem ansonsten symmetrischen Flugzeitspektrum der Photodissoziation von $\mathrm{HI} / \mathrm{HCl}$ nicht separieren läßt.

\subsubsection{Orientierung und Laserpolarisation}

Um gebildete $\mathrm{HRgX}$ Moleküle in der hier verwendeten Apparatur nachweisen zu können, spielt die Anisotropie der Polarisierbarkeit des Moleküls, sein Dipolmoment, die Leistungsdichte des Laserfeldes und die Feldstärke des elektrostatischen Abzugsfeldes im WMTOFMS eine Rolle. Diese Parameter legen den Grad der Orientierung des Moleküls fest. Diese Orientierung wiederum verursacht die Asymmetrie in den Flugzeitspektren, die zur Identifikation dieser Moleküle ausgenutzt wird.

Den Orientierungsgrad gibt der sogenannte Richtungskosinus $\left\langle\cos \theta_{S}\right\rangle$ im elektrostatischen Feld $E_{S}$ an. Die Berechnung der Kerngrößen $\omega$ und $\Delta \omega$ geht aus Gl. 1.47 hervor. Die zugehörigen Moleküldaten sind in Tab. 5.3 und 1.4 aufgeführt. Die experimentellen Bedingungen wie statische elektrische Feldstärke und Leistungsdichte des Laserfeldes sind im Abschnitt 4.4 beschrieben. Daraus läßt sich mit Hilfe der Rechnungen von B. Friedrich der Grad der Orientierung bestimmen [Nah02]. Die graphische Ermittlung geht aus Abb. 5.12 hervor und die Ergebnisse sind in Tab. 5.3 zusammengefaßt. Von einer Orientierung in eine Richtung spricht man, wenn gilt $\left\langle\cos \theta_{S}\right\rangle>0.8$ [Fri99b].

Die Molekül- und experimentellen Parameter reichen für eine Orientierung der drei hier diskutierten Systeme aus $\left(\left\langle\cos \theta_{S}\right\rangle \geq 0.93\right)$. Zwei Fragen sind allerdings noch unbeantwor- 
tet:

1. Welchen Einfluß hat der Winkel zwischen Laserfeld und elektrostatischem Feld auf den Orientierungsgrad?

2. Der erste angeregte Pendelzustand $|\tilde{J} M\rangle=1,0$ führt zur genau entgegengesetzten Orientierung der Moleküle wie der Grundzustand $|\tilde{J} M\rangle=0,0$. Warum wird der erste angeregte Pendelzustand nicht bevölkert, der energetisch nur durch einige $\mathrm{mK}$ vom Grundzustand getrennt ist?

Die erste Frage stellt sich, weil es bisher nur Berechnungen zur Orientierung in kombinierten Feldern gibt, wenn die Laserpolarisation parallel zum Vektor der elektrischen Feldstärke ausgerichtet ist $\left(E_{L} \| E_{S}\right)$. Experimentell ist aber die Orientierung bei $\mathrm{HXel}$ sowohl für $E_{L} \| E_{S}$ als auch $E_{L} \perp E_{S}$ beobachtet worden. Dagegen hat bei der Photodissoziation von $\mathrm{HCl}$ auf Xe Clustern eine Feldausrichtung $E_{L} \perp E_{S}$ keine Asymmetrie in den Flugzeitspektren verursacht. Für die Photodissoziation von $\mathrm{HBr}$ auf $\mathrm{Xe}$, wo bisher nur eine Messung für $E_{L} \perp E_{S}$ existiert, ist eine leichte, asymmetrische Intensitätserhöhung im vorderen Teil des Flugzeitspektrums zu erkennen, was als Indiz für die Bildung, Orientierung und den Nachweis von $\mathrm{HXeBr}$ aufgefaßt wird. Weil es zu diesen unterschiedlichen Feldausrichtungen noch keine Rechnungen gibt, kann diese Fragestellung im folgenden nur qualitativ erörtert werden.

HXel erreicht aufgrund seiner sehr hohen Anisotropie in der Polarisierbarkeit gepaart mit der sehr kleinen Rotationskonstante den höchsten Ausrichtungsgrad im Laserfeld. Diese stark ausgerichteten Moleküle könnten durch eine kleine äußere Störung in Form eines schwachen elektrostatischen Feldes umgeklappt und in diesem Feld orientiert werden. Dieser Effekt wäre nach den Messungen genauso stark wie bei parallelen Feldern. Bei $\mathrm{HXeBr}$ liegen andere Bedingungen vor. Man erreicht hier eine schwächere Ausrichtung. Dadurch gelingt es anscheinend nur noch, einen Teil der Moleküle umzuklappen und im elektrostatischen Feld zu orientieren. $\mathrm{HXeCl}$ mit seiner deutlich geringeren Ausrichtung im Laserfeld $(\Delta \omega(\mathrm{HXeI})=225$ vs. $\Delta \omega(\mathrm{HXeCl})=80)$ kann bei zueinander senkrechten Feldern nicht mehr umgeklappt und orientiert werden. In diesem Rahmen ist unbedingt die Messung zur Photodissoziation von $\mathrm{HBr}$ auf Xe Clustern mit $\left(E_{L} \| E_{S}\right)$ nachzuholen, um Klarheit über die Eigenschaften von $\mathrm{HXeBr}$ zu erlangen.

Die zweite Frage nach der Bevölkerung der Pendelzustände führt zur Diskussion über den Einfluß der Clusterumgebung nicht nur auf die Bildung sondern auch auf die Orientierung der HRgX Moleküle. 


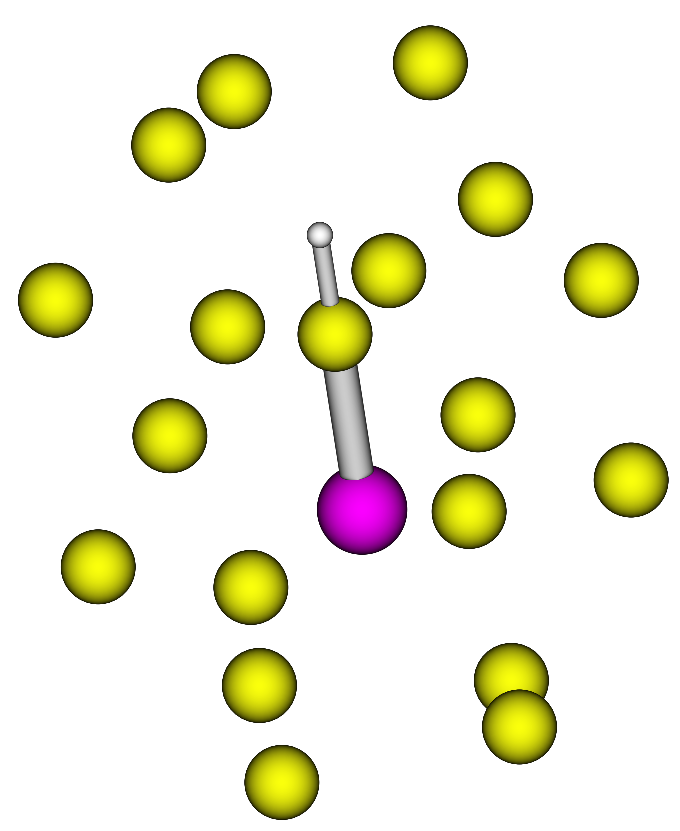

Abbildung 5.13: Von Z. Bihary berechnete Struktur zur geometrischen Anordnung von $\mathrm{HXel}$ in einem $\mathrm{Xe}_{18}$ Cluster [Nah02].

Rechnungen zur Konfiguration von $\mathrm{HXel}$ in einer Xe Matrix haben gezeigt, daß das HXel Molekül in einem sehr starren Käfig sitzt [Bih02, Pet02b]. In einer Kooperation zwischen unserer Gruppe und der Gruppe von R.B. Gerber hat Z. Bihary Rechnungen zur Struktur und Rotation von $\mathrm{HXel}$ in $\mathrm{Xe}_{18}$ Clustern durchgeführt [Nah02]. Die Untersuchung der lokalen Schwingungen des Moleküls im Xe Cluster führt zu dem Ergebnis, daß die Biegeschwingung eine Amplitude von $10^{\circ}$ und die Kippschwingung (Libration) eine von weniger als $2^{\circ}$ besitzt. Für eine Rotationsanregung spielt einzig die Kippschwingung eine Rolle, die aber bei dieser Amplitude zeigt, daß das HXel Molekül starr in dem kalten Cluster sitzt. Wenn das Molekül den Cluster verläßt, bzw. der Cluster aufgrund der frei werdenden Dissoziationsenergie und Solvatisierungsenergie (HXel im Xe Cluster: $1.2 \mathrm{eV}$ ) fragmentiert, sind Rotationszustände nur mit einer sehr geringen Wahrscheinlichkeit besetzt. Wegen der Größe der Xe Cluster ist es durchaus denkbar, daß sich noch ein Xe Atom am HXel Molekül befindet. Für diesen Cluster HXel-Xe wurden von Z. Bihary die Trägheitsmomente in Abhängigkeit von der Konfiguration berechnet. Daraus resultieren die Rotationskonstanten $0.026 \mathrm{~cm}^{-1}, 0.011 \mathrm{~cm}^{-1}$ und $0.008 \mathrm{~cm}^{-1}\left(\mathrm{HXel}: 0.026 \mathrm{~cm}^{-1}\right)$. Moleküle mit kleinerer Rotationskonstante lassen sich in elektrischen Feldern besser orientieren. Wie 
sich dies letztendlich auf den Grad der Orientierung auswirkt, kann nur die Berechnung der Anisotropie in der Polariserbarkeit von HXel-Xe zeigen.

\subsubsection{Potentiale, Schwingungen und Dissoziation}

Die Bildung und Orientierung der Spezies HRgX ist theoretisch sowie experimentell gut erforscht. In den Experimenten dieser Arbeit gelingt der Nachweis dieser Moleküle über die Detektion der Wasserstofffragmente aus der Dissoziation von HRgX. Damit stellt sich die Frage nach den Dissoziationsmechanismen.

- Befinden sich die Moleküle HRgX nach der Bildung im Grundzustand oder in einem schwingunsangeregten Zustand?

- Zerfällt das Molekül durch Dissoziation hervorgerufen durch eine Potentialkreuzung zwischen schwingungsangeregtem und repulsivem Zustand?

- Gelangt das Molekül durch Absorption eines Photons in eine elektronisch angeregten Zustand $(\mathrm{HRgX})^{*}$ aus dem es in $\mathrm{H}+(\operatorname{Rg} X)^{*}$ dissoziiert?

Aus Ermangelung an Rechnungen und Pump-Probe Messungen zur Dissoziation der Moleküle kann diese Diskussion nur qualitativ das für und wider der obigen Thesen beleuchten.

Aufschluß über den Zustand, in dem sich die Moleküle nach ihrer Bildung befinden, kann die gemessene Energieverteilung der Wasserstofffragmente geben. Die Bindungsenergien der elektronischen Grundzustände von $\mathrm{HXel}$ und $\mathrm{HXeCl}$ betragen $0.42 \mathrm{eV}$ bzw. $0.9 \mathrm{eV}$. Die Energieverteilung aus der Dissoziation von $\mathrm{HXel}$ erstreckt sich über den Bereich 0 $0.4 \mathrm{eV}\left(0-3300 \mathrm{~cm}^{-1}\right)$ mit einem Maximum zwischen 100 und $120 \mathrm{meV}\left(830-1000 \mathrm{~cm}^{-1}\right)$. Bei $\mathrm{HXeCl}$ deckt die Verteilung den Energiebereich $0-0.7 \mathrm{eV}\left(0-5800 \mathrm{~cm}^{-1}\right)$ ab. Das Maximum befindet sich bei $180 \mathrm{meV}\left(1500 \mathrm{~cm}^{-1}\right)$. Die Moleküle sind nach der Orientierung und während der Dissoziation nicht mehr von dem Xe Cluster umgeben. Dieser ist auf dieser Zeitskala bereits fragmentiert. Die Wasserstofffragente stammen somit aus der Dissoziation eines freien Moleküls und sind unbeeinflußt vom Käfigeffekt. Daraus folgt, daß eine breite energetische Verteilung der Wasserstofffragmente nur aus einer Verteilung der Moleküle in unterschiedliche Anfangszustände resultieren kann. Hierzu kommen aufgrund der oben diskutierten verhinderten Rotationen nur Schwingungsanregungen in Frage. Gerechnete und aus Matrixexperimenten bekannte Schingungsanregungen sind in Tab. 5.4 aufgeführt. 


\begin{tabular}{|lrcc|}
\hline Schwingung & & $\mathbf{H X e C l}$ & HXel \\
\hline \hline Xe-X Streck & $\nu_{1}$ & $263^{1}$ & $146^{2}$ \\
& $2 \nu_{1}$ & & $283^{2}$ \\
& $3 \nu_{1}$ & & $424^{2}$ \\
H-Xe-X Biege & $\nu_{2}$ & $547^{1}$ & $450^{2}$ \\
& $2 \nu_{2}$ & $\approx 1050-1200$ & $896-1000^{2}$ \\
Xe-H Streck & $\nu_{3}$ & $1649^{1}$ & $1193^{2}$ \\
\hline
\end{tabular}

Tabelle 5.4: Schwingungsfrequenzen in $\mathrm{HXel}$ und $\mathrm{HXeCl}$ in $\mathrm{cm}^{-1}$. Referenzen: ${ }^{1}$ [Joh99], ${ }^{2}[$ Lun00b].

Es spricht viel dafür, wenn man die Maxima der Energieverteilungen mit den Schwingungsfrequenzen vergleicht, daß in den Molekülen die $\mathrm{H}-\mathrm{Xe}-\mathrm{X}$ Biegeschwingung $\left(2 \nu_{2}\right)$ oder die $\mathrm{Xe}-\mathrm{H}$ Streckschwingung $\left(\nu_{3}\right)$ angeregt ist. Höhere Schwingungsfrequenzen sind durch Überlagerungen der aufgeführten Anregungen bzw. durch deren Obertöne möglich.

Die Energieverteilungen zur Dissoziation von $\mathrm{HXeX}$ aus dieser Arbeit unter Berücksichtigung der Matrixexperimente und -rechnungen sprechen dafür, daß die Moleküle nach ihrer Bildung schwingungsangeregt sind und eine repulsive Potentialkreuzung zur Dissoziation in $\mathrm{H}+\mathrm{XeX}$ führt.

Allerdings sollte man den Aspekt der Photodissoziation dieser Moleküle nicht außer acht lassen. Eine direkte Photoanregung aus dem Grundzustand heraus in das Dissoziationskontinuum findet nicht statt. Dies würde zu Wasserstofffragmenten mit Energien zwischen 4.5 und $6.2 \mathrm{eV}$ führen, die in den Messungen nicht beobachtet werden. Rechnungen zu den $\mathrm{HXeCl}$ Potentialflächen und elektronischen Anregungen zeigen einen möglichen Übergang in einen gebundenen $(\mathrm{HXeCl})^{*}$ Zustand, der durch $5.4 \mathrm{eV}$ vom Grundzustand getrennt ist [Joh99]. Seine Bindungsenergie beträgt ungefähr $1.4 \mathrm{eV}$, so daß er sich mit der Laserstrahlung in unserem Experimnt $(193 \mathrm{~nm}, 6.43 \mathrm{eV})$ bevölkern läßt. Aufgrund der Überschußenergie und der Besetzung von Schwingungszuständen im elektronischen Grundzustand führt dies zur Anregung unterschiedlicher Schwingungszustände im elektronisch angeregten Zustand. Dies hat nach dem oben beschriebenen Mechanisumus eine Dissoziation in $\mathrm{H}+(\mathrm{XeCl})^{*}$ zur Folge. 



\section{Zusammenfassung und Ausblick}

Im Zentrum der hier vorgestellten Experimente steht die Photodissoziation von Chlor-, Brom- und lodwasserstoffmolekülen in Edelgasclusterumgebungen. Die Moleküle befinden sich durch Koexpansion im Cluster oder durch Pick-up in der Nähe der Oberfläche des Clusters. Die weit fortgeschrittenen theoretischen Berechnungen auf diesem Gebiet werden zu qualitativen und quantitativen Vergleichen mit den experimentellen Ergebnissen herangezogen.

\section{Käfigeffekt}

Die Photodissoziation von mittels Pick-up an Ar Cluster angelagerten Halogenwasserstoffen zeigt, daß die Position in der Umgebung der Oberfläche eine entscheidende Auswirkung auf die Energieverteilung der Wasserstofffragmente hat.

Das HI Molekül sitzt überwiegend in der zweiten Schale des Ar Clusters, wo es beliebig orientiert sein kann. Die beiden Extremrichtungen lassen das Wasserstoffatom zum Zentrum des Clusters oder nach außen zeigen. Der erste Fall führt zu vollständig abgebremsten Wasserstofffragmenten, der zweite zu erhöhtem Käfigausgang. Die zur Seite fliegenden Wasserstofffragmente werden teilweise abgebremst.

Dagegen sitzen $\mathrm{HBr}$ Moleküle zu gleichen Teilen in der äußeren und der zweiten Schale des Clusters. Das Flugzeitspektrum wird von vollständig abgebremsten und frei austretenden Wasserstofffragmenten dominiert. Erstere resultieren aus einer Orientierung des Wasserstoffatoms in Richtung des Clusterzentrums, die nach der Dissoziation den gesamten Cluster durchqueren müssen. Letztere resultieren aus der umgekehrten Orientierung, was dazu führt, daß nur eine schwache Wechselwirkung mit dem Käfig eingegangen wird. Die Variation der Ar Clustertemperatur kann zu einer leichten Veränderung der Eindringtiefe der $\mathrm{HBr}$ Moleküle im Pick-up Prozeß führen.

Chlorwasserstoff ist derart in die Oberfläche des Ar Clusters eingebettet, daß eine Bewegung des Moleküls kaum möglich ist. Das Wasserstoffatom zeigt zum Clusterzentrum 
und muß nach der Dissoziation den gesamten Cluster durchqueren. Das Flugzeitspektrum wird von vollständig abgebremsten Wasserstofffragmenten dominiert.

Die Experimente zeigen, daß dieses Verhalten unabhängig von der Clustergröße ist. Die Wahl des Edelgasclusters hat allerdings einen deutlichen Einfluß auf den Käfigeffekt. Während Pick-up von Halogenwasserstoffen an Ar Cluster dazu führt, daß sich das Molekül in der Nähe der Oberfläche befindet, dringt es bei Ne Clustern unabhängig von der Größe vollständig in diesen ein. Experimentell ist dies im Größenbereich $100<\langle n\rangle<1600$ für $\mathrm{HBr}$ und $\mathrm{HCl}$ gezeigt worden.

In der Koexpansion von $\mathrm{HBr}$ mit Ar wird das $\mathrm{HBr}$ Molekül in das Zentrum des Ar Clusters eingelagert. In diesem Fall ist der Käfigeffekt stark von der Clustergröße abhängig. Für größere Ar Cluster $(\langle n\rangle=450)$ gibt es keine Wasserstofffragmente, die den Cluster direkt verlassen. Der Vergleich mit Rechnungen, die dies für eine Größe von $n=146$ voraussagen, zeigt unter Berücksichtigung der Größenverteilung im Experiment auch quantitativ gute Übereinstimmung.

Im Gegensatz zu $\mathrm{HBr}$ Molekülen in Ar Clustern zeigt die Photodissoziation von kleinen $\mathrm{HCl}$ Clustern $(\langle n\rangle=7)$ eingebettet in Ar Clustern $(23<\langle n\rangle<185)$ deutlichen Käfigausgang. Dies ist ein kinematischer Effekt: Weil die Überschußenergie des Wasserstofffragments aus der Dissoziation von $\mathrm{HCl}$ um $55 \%$ höher ist als bei $\mathrm{HBr}$, besteht eine höhere Wahrscheinlichkeit für den freien Käfigausgang. Für kleiner werdende Ar Clustergrößen nimmt der direkte Austritt zu. Bei reinen $\mathrm{HCl}$ Clustern $(\langle n\rangle=6)$ führt die Kombination der hohen kinetischen Energie der Wasserstofffragmente gepaart mit der offenen Clusterstruktur zu $100 \%$ Käfigausgang.

\section{Winkelverteilung der Fragmente}

In diesen Experimenten konnte gezeigt werden, daß die Clusterumgebung einen signifikanten Einfluß auf die Fragmentwinkelverteilung aus der Photodissoziation von Halogenwasserstoff-Molekülen besitzt.

Während die Dissoziation des freien HI Moleküls eine klare Anisotropie der Produktkanäle im Grund- und angeregten Zustand ( $\beta=-1$ und $\left.\beta^{*}=2\right)$ zeigt, wird die Winkelverteilung der Wasserstofffragmente aus der Dissoziation von $\mathrm{HI}$ in der zweiten Schale von Ar Clustern durch Streuung und Stöße in der Umgebung nahezu isotrop ( $\beta=0$ und $\left.\beta^{*}=0.3\right)$.

Das freie $\mathrm{HCl}$ Molekül kann nur über eine senkrechte Anregung photodissoziiert werden. Die Ergebnisse dieser Arbeit zeigen eine vollständige Isotropie in der Winkelverteilung der 
abgebremsten Wasserstofffragmente aus der Photodissoziation von $\mathrm{HCl}$ eingebettet in $\mathrm{Ar}$ Cluster. Die Ar Atome verändern die Fragmenttrajektorien durch eine Vielzahl an Stößen derart, daß jegliche Information über die Startrichtung der Wasserstofffragmente verloren geht.

\section{$\mathrm{HXel}$ und $\mathrm{HXeCl}$}

In dieser Arbeit konnte bewiesen werden, daß sich Moleküle mit einer hohen Polarisierbarkeit in kombinierten starken gepulsten Laserfeldern und schwachen elektrostatischen Feldern orientieren lassen. Dies wird benutzt, um die Bildung der ionisch gebundenen, stabilen Moleküle $\mathrm{HXel}$ und $\mathrm{HXeCl}$ im Anschluß an die Photodissoziation von $\mathrm{HI}$ und $\mathrm{HCl}$ in einer Xe Clusterumgebung nachzuweisen. Die Präparation in den Pendel-Grundzustand, der energetisch nur um $10 \mathrm{mK}$ vom ersten angeregten Zustand mit umgekehrter Orientierung getrennt ist, gelingt durch Verhinderung von Rotationen im Xe Cluster. Der Beweis hierfür konnte durch Umkehrung der elektrischen Feldrichtung im Wiley-McLaren Flugzeitmassenspektrometer geführt werden.

Erste Messungen sprechen auch für die Bildung und Orientierung von $\mathrm{HXeBr}$. Dies soll in weiteren Experimenten verifiziert werden. Ein weiteres Ziel ist der Nachweis von $\mathrm{HKrCl}$. Die Moleküle $\mathrm{HKrl}, \mathrm{HKrBr}$ und $\mathrm{HArCl}$ konnten bisher weder in der Gasphase noch in Matrizen gebildet werden.

\section{Ausblick}

Die experimentelle Methode dieser Arbeit ist nicht auf Halogenwasserstoffe und Edelgascluster beschränkt. In diesem Zusammenhang ist die Photodissoziation von Wasser in Edelgasund Wasserclustern sowie die Photodissoziation von $\mathrm{HCl}$ und $\mathrm{HBr}$ in Wasserclustern sehr interessant. Bei letzteren kann insbesondere die lonenpaarbildung $\mathrm{H}_{3} \mathrm{O}^{+}+\mathrm{Cl}^{-} / \mathrm{Br}^{-}$in endlichen Systemen untersucht werden [AH02]. Es sind aber auch ähnliche Versuche mit weiteren Molekülen denkbar, die eine zentrale Rolle in der Atmosphärenchemie spielen $\left(\mathrm{HOCl}, \mathrm{OClO}, \mathrm{ClONO}_{2}\right)$. Dabei steht die Frage nach den Reaktionskanälen, insbesondere der Produktion von $\mathrm{Cl}$ im Vordergrund [Kre99].

Experimentelle Veränderungen sind hier bezüglich der Dissoziations- und Nachweiswellenlänge vorzunehmen. Der vorhandene Excimerlaser läßt sich sowohl bei $193 \mathrm{~nm}$ als auch bei $157 \mathrm{~nm}$ betreiben. Die Ionisation von Fragmenten wie $\mathrm{Cl}$ und $\mathrm{OCl}$ geschieht idealerweise mit VUV Laserstrahlung zwischen $70 \mathrm{~nm}$ und $90 \mathrm{~nm}$. Licht dieser Wellenlänge kann mit resonanter Summenfrequenzmischung an einem Xe Düsenstrahl erzeugt werden. Die 
Trennung des Lichts unterschiedlicher Wellenlängen geschieht an hochreflektiven Spiegeln für VUV Licht [Sof87].

Im weiteren sind zeitaufgelöste Experimente zur Photodissoziation von $\mathrm{HOCl}, \mathrm{OCIO}$ und $\mathrm{ClONO}_{2}$ in großen, neutralen Clustern geplant. Dies ermöglicht Vergleiche der nichtredundanten, komplementären Meßgrößen in der Zeit- und Frequenzdomäne. Die Untersuchung der Produktkanäle aus der Photodissoziation erfolgt durch Femtosekunden PumpProbe Spektroskopie des Muttermoleküls und der Fragmente. Diese Experimente sollen in einer Kooperation mit der Gruppe von B. Abel durchgeführt werden [Cha01, AB02].

Nicht nur auf dem Gebiet der Atmosphärenchemie können mit der experimentellen Methode dieser Arbeit Fortschritte erzielt werden. Auch die Einwirkung von Licht auf biologische Moleküle in Wasserclustern oder anderen Lösungsmitteln kann untersucht werden. 


\section{Abbildungsverzeichnis}

1.1 Winkelverteilung bei der Photodissoziation zweiatomiger Moleküle . . . 9 9

1.2 Winkelverteilung der Fragmente bei der Photodissoziation . . . . . . . . . 11

1.3 Potentialkurven für Halogenwasserstoffe . . . . . . . . . . . . . . . 13

1.4 Photonen-Absorptionsquerschnitte der Halogenwasserstoffe . . . . . . . . 15

1.5 Schematische Darstellung des Käfig-Effekts . . . . . . . . . . . . . . . 19

1.6 Ikosaeder-Strukturen von Edelgasclustern . . . . . . . . . . . . . . . . . 29

1.7 Einfluß der turn-around-Zeit auf Flugzeitspektren . . . . . . . . . . . 30

1.8 Wiley-McLaren Flugzeitmassenspektrometer . . . . . . . . . . . . . . 31

1.9 Photoionisation von Wasserstoffatomen . . . . . . . . . . . . . 38

1.10 Bildung von $\mathrm{HRgX}$ in der Photodissoziation von $\mathrm{HX} \ldots \mathrm{Rg} \ldots 40$

1.11 Ausrichtung und Orientierung von Molekülen in äußeren Feldern . . . . . 42

2.1 Schematischer Aufbau des Experiments . . . . . . . . . . . . . . . . 48

2.2 Molekularstrahlapparatur . . . . . . . . . . . . . . . . . . . . . . . . 49

2.3 Quelle zur Erzeugung großer Ne Cluster . . . . . . . . . . . . . . . . 52

2.4 Lasersystem zur Erzeugung von $243 \mathrm{~nm} \ldots \ldots$. . . . . . . . . . . 57

2.5 Gespühlter/evakuierter Strahlengang für $193 \mathrm{~nm} \ldots \ldots$. . . . . . . . . . 59

2.6 Einkopplung der Polarisationen $0^{\circ}$ und $90^{\circ} \ldots \ldots$. . . . . . . . . 61

2.7 Polarisationsdreher für $193 \mathrm{~nm} \ldots \ldots \ldots 6$

2.8 Aufbau Flugzeitmassenspektrometer kurz . . . . . . . . . . . . . . . 64

2.9 Aufbau des lonendetektors (MCP) mit Signalauskopplung . . . . . . . . . 65

2.10 Beschaltung der elektischen Felder im WMTOFMS . . . . . . . . . . . 66

2.11 Zeitliche Pulsfolge des Dissoziationslasers und des elektrischen Feldes im WMTOFMS ........................... 67

2.12 Aufbau Flugzeitmassenspektrometer lang . . . . . . . . . . . . . . . . . 69 
2.13 Aufbau des lonendetektors (MSP) mit Signalauskopplung . . . . . . . . 70

2.14 Signalverstärkung durch MSP-Detektor . . . . . . . . . . . . . . . . 70

2.15 Massenspektrum im Hochfeld-Modus mit $193 \mathrm{~nm}$ als Dissoziations- und lonisationswellenlänge . . . . . . . . . . . . . . . . . . 71

3.1 Detektionswahrscheinlichkeit im WMTOFMS . . . . . . . . . . . . 74

3.2 Meßprinzip zur Dissoziation . . . . . . . . . . . . . . . . . . 76

3.3 Transformation eines Flugzeitspektrums in den Energieraum . . . . . . . 79

3.4 Ablaufplan zum Programmpaket der Trajektoriensimulation . . . . . . . . 81

3.5 Monoenergetisches Flugzeitspektrum für die Photodissoziation von $\mathrm{HBr}$ ermittelt mit dem MC-Trajektorien-Simulationsprogramm . . . . . . . . 83

3.6 Monoenergetisches Flugzeitspektrum für die Photodissoziation von $\mathrm{HCl}$ ermittelt mit dem MC-Trajektorien-Simulationsprogramm . . . . . . . . . 84

3.7 Photodissoziation von $\mathrm{HI}$ Molekülen bei 243nm . . . . . . . . . . . . 84

4.1 Photodissoziation von $\mathrm{HI}$ auf $\operatorname{Ar}_{139} \ldots \ldots . \ldots 9 . \ldots . \ldots 9$

4.2 Photodissoziation von HI: Vergleich Molekül - Ar Clusterumgebung . . . 91

4.3 Libration von $\mathrm{HBr}$ in $\mathrm{Ar}_{n} \ldots \ldots . \ldots . \ldots 93$

4.4 Flugzeitspektrum von $\mathrm{HBr}$ auf geheizten Ar Clustern konstanter Größe . . 94

4.5 Käfig-Effekt von $\mathrm{HBr}$ auf $\mathrm{Ar}$ Clustern in Abhängigkeit von der Clustertemperatur . . . . . . . . . . . . . . . . . . 96

4.6 Photodissoziation von $\mathrm{HBr}$ in großen Ar Clustern . . . . . . . . . . . 97

4.7 Photodissoziation von einem $\mathrm{HBr}$ Molekül auf Ne Clustern unterschiedlicher Größe . . . . . . . . . . . . . . . . . . . . . . . . . 101

4.8 Energieverteilungen der Messungen $\mathrm{HCl}$ auf Edelgasclustern . . . . . . . . 103

4.9 Größenabhängigkeit der Photodissoziation von $\mathrm{HCl}$ auf $\mathrm{Ne}_{\mathrm{n}}$ Clustern . . . 105

4.10 Größenabhängigkeit der Photodissoziation von $\mathrm{HCl}$ auf $\mathrm{Ar}_{\mathrm{n}}$ Clustern . . . 106

4.11 Photodissoziation von $(\mathrm{HCl})_{x}-\operatorname{Ar}_{n},\langle x\rangle \approx 8,\langle n\rangle=0-185 \ldots . . . .109$

4.12 Photodissoziation von $(\mathrm{HCl})_{x}$ in $\mathrm{Ar}_{\mathrm{n}}$ in Abhängigkeit von der Laserpolarisation 110

4.13 Flugzeitspektren zur Photodissoziation von HI auf Xe Clustern . . . . . . 112

4.14 Energieverteilung der Wasserstofffragmente aus der Phtotodissoziation von $\mathrm{HI}$ auf $\mathrm{Xe}$ Clustern . . . . . . . . . . . . . . . . . . . . . . 113

4.15 Wasserstofffragment-Energieverteilung aus der Dissoziation von orientier-

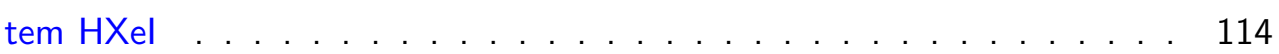

4.16 Nachweis von HXel bei umgekehrter Feldrichtung im WM-TOFMS . . . . 115 
4.17 Photodissoziation von $\mathrm{HCl}$ auf $\mathrm{Xe}_{\mathrm{n}}$ : Polarisation $0^{\circ}$. . . . . . . . . . . . 118

4.18 Photodissoziation von $\mathrm{HCl}$ auf $\mathrm{Xe}_{\mathrm{n}}$ : Polarisation $90^{\circ}$. . . . . . . . . . . . 120

5.1 Mögliche Positionen eines Wasserstoffhalogenids in einem Edelgascluster . 122

5.2 Aufenthaltsort von $\mathrm{HBr}$ und $\mathrm{HI}$ Molekülen in Ar Clustern . . . . . . . . . . . 124

5.3 Photodissoziation von $\mathrm{HBr}$ auf Ar Clustern: Vergleich zwischen Experiment und Rechnung . . . . . . . . . . . . . . . . . 126

5.4 Photodissoziation von HBr Molekülen in Ne Clustern: Experiment und Theorie127

5.5 Berechnungen zur Photodissoziation von $\mathrm{HBr}$ in Ar Clustern . . . . . . . 131

5.6 Rechnungen zu HCl Molekülen auf $\mathrm{Ar}_{54}$ Clustern an unterschiedlichen Positionen ........................... 133

5.7 Berechnete Energieverteilung zur Photodissoziation von $\mathrm{HCl}$ in $\mathrm{Ar}_{54}$ Clustern135

5.8 Minimumskonfiguration von $\mathrm{HCl}_{\mathrm{n}}, \mathrm{n}=3,6$ und 10 . . . . . . . . . . . 136

5.9 Photodissoziation von $\mathrm{HI}$ auf Ar Clustern: Experiment und Theorie . . . . 138

5.10 Einfluß der Clusterumgebung auf die Winkelverteilung der Fragmente . . 140

5.11 Anisotropie in der Photodissoziation von $\mathrm{HCl}$ in einem $\mathrm{Ar}_{12}$ Cluster . . . . 142

5.12 Orientierungsgrad in kombinierten Laser- und elektrostatischen Feldern . . 144

5.13 Struktur von $\mathrm{HXel}-\mathrm{Xe}_{18}$. . . . . . . . . . . . . . . . . . . . . . . . . . 147 



\section{Tabellenverzeichnis}

1.1 Anisotropie und Verzweigungsverhältnis von $\mathrm{HCl}, \mathrm{HBr}$ und $\mathrm{HI} \ldots \ldots$

1.2 Charakteristische Größen der verwendeten Edelgase . . . . . . . . . . . 25

1.3 lonisierungsenergien und -wellenlängen von Atomen und Molekülen . . . . 35

1.4 Moleküldaten von $\mathrm{HXeX}, \mathrm{X}=\mathrm{I}, \mathrm{Br}, \mathrm{Cl}$; und $\mathrm{HI} \ldots \ldots . . . . . .39$

2.1 Vakuumerzeugung an der Molekularstrahlapparatur . . . . . . . . . . 50

2.2 Kenndaten der Lasersysteme . . . . . . . . . . . . . . . . 56

3.1 Daten zur Photodissoziation der Wasserstoffhalogenide . . . . . . . . . 82

4.1 Daten zur Photodissoziation von $\mathrm{HI}$ auf Ar Clustern . . . . . . . . . . . 89

4.2 Koexpansion von $\mathrm{HBr}$ in Argon . . . . . . . . . . . . . . . . . . 98

4.3 Daten zur Photodissoziation von $\mathrm{HBr}$ adsobiert an $\mathrm{Ne}$ Cluster . . . . . . 99

4.4 Strahldaten zur Erzeugung der Edelgascluster für Pick-up Experimente mit

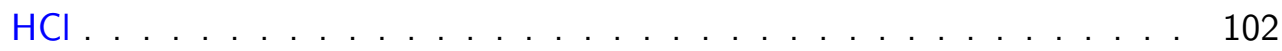

4.5 Experimentelle Parameter zur Photodissoziation von $\mathrm{HCl}$ angelagert an $\mathrm{Ne}$ Cluster unterschiedlicher Größe . . . . . . . . . . . . . . . . . . 104

4.6 Experimentelle Parameter zur Photodissoziation von $\mathrm{HCl}$ angelagert an $\mathrm{Ar}$ Cluster unterschiedlicher Größe . . . . . . . . . . . . . . . . . 105

4.7 Daten zur $(\mathrm{HCl})_{x} \mathrm{Ar}_{\mathrm{n}}$ Clustererzeugung . . . . . . . . . . . . . . 108

4.8 Käfigeffekt in der Photodissoziation von $(\mathrm{HCl})_{x}-\mathrm{Ar}_{\mathrm{n}} \ldots \ldots . . . . .110$

5.1 Charakterisierung der Ar Clustergröße und Vergleich von Experiment und Theorie . . . . . . . . . . . . . . . . . . . . . 130

5.2 Dissoziationsenergien von $\mathrm{HRgX}$ Molekülen . . . . . . . . . . . . . 143

5.3 Daten zur Orientierungsgrad von $\mathrm{HXel}, \mathrm{HXeBr}, \mathrm{HXeCl}$ und $\mathrm{HI} \ldots 145$

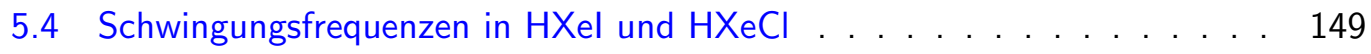





\section{Literaturverzeichnis}

[AH02] A. Al-Halabi, R. Bianco und J.T. Hynes. Acid Dissociation of $\mathrm{HBr}$ on a Model Ice Surface. J. Phys. Chem. A 106 (2002) 7639.

[Aho00] J. Ahokas, K. Vaskonen, J. Eloranta und H. Kunttu. Electronic Absorption Spectra of HXeCl, HXeBr, HXel, and HXeCN in Xe Matrix. J. Phys. Chem. A 104 (2000) 9506

[Ale88] M.L. Alexander, N.E. Levinger, M.A. Johnson, D. Ray und W.C. Lineberger. Recombination of $\mathrm{Br}_{2}^{-}$photodissociated within mass selected ionic clusters. J. Chem. Phys. 88 (1988) 6200.

[Ale93] M.H. Alexander, B. Pouilly und T. Duhoo. Spin-Orbit Branching in the Photofragmentation of HCl. J. Chem. Phys. 99 (1993) 1752.

[Ale98] M.H. Alexander, X. Li, R. Liyanage und R.J. Gordon. Spin-orbit branching in the predissociation of the $\mathrm{C}^{1} \Pi$ state of $\mathrm{HCl}$ and $\mathrm{DCl}$ : a manifestation of quantum interference. Chem. Phys. 231 (1998) 331.

[Ale00] A.B. Alekseyev, H.-P. Liebermann, D.B. Kokh und R.J. Buenker. On the ultraviolet photofragmentation of hydrogen iodide. J. Chem. Phys. 113 (2000) 6174.

[Apk99] V.A. Apkarian und N. Schwentner. Molecular Photodynamics in Rare Gas Solids. Chemical Reviews 99 (1999) 1481.

[AB02] J. ABmann, A. Charvat, D. Schwarzer, C. Kappel, K. Luther und B. Abel. Real-Time Observation of Intra- and Intermolecular Vibrational Energy Flow of Selectively Excited Alkyl lodides in Solution: The Effect of Chemical Substitution. J. Phys. Chem. A 106 (2002) 5197.

[Asc99] D. Ascenzi, P.M. Regan und A.J. Orr-Ewing. The ultraviolet photodissociation of DCl: $\mathrm{H} / \mathrm{D}$ isotope effects on the $\mathrm{Cl}\left({ }^{2} \mathrm{P}\right)$ atom spin-orbit branching. Chem. Phys. Lett. 310 (1999) 477.

[Ash96] M.N.R. Ashfold, D.H. Mordaunt und S.H.S. Wilson. Photodissciation Dynamics of Hydride Molecules: H Atom Photofragment Translational Spectroscopy. In D.C. 
Neckers, D.H. Volman und G. von Bünau, Hg., Advances in Photochemistry, Bd. 21 (John Wiley \& Sons, New York, 1996) (Seiten 217-295).

[Bal01] N. Balakrishnan, A.B. Alekseyev und R.J. Buenker. Ab inition quantum mechanical investigation of the photodissociation of HI and DI. Chem. Phys. Lett. 341 (2001) 594.

[Bas75] S. Bashkin und J. O. Stoner. Atomic Energy Levels and Grotrian Diagrams (North-Holland Publishing Company, 1975).

[Bau95] R. Baumfalk. UV-Photodissoziation von Molekülclustern bekannter Größenverteilung. Diplomarbeit, Universität Göttingen (1995).

[Bau97a] R. Baumfalk, U. Buck, C. Frischkorn, S.R. Gandhi und C. Lauenstein. Photodissociation and Size Analysis of $\mathbf{( H B r})_{n}$ Clusters. Ber. Bunsenges. Phys. Chem. 101 (1997) 606.

[Bau97b] R. Baumfalk, U. Buck, C. Frischkorn, S.R. Gandhi und C. Lauenstein. UV Photolysis of $(\mathbf{H B r})_{n}$ Clusters with Known Size Distribution. Chem. Phys. Lett. 269 (1997) 321.

[Bau99a] R. Baumfalk. Photodissoziation von Halogenwasserstoffen in und auf Edelgasclustern. Dissertation, Bericht 12/1999, MPI für Strömungsforschung, Göttingen (1999).

[Bau99b] R. Baumfalk, U. Buck, C. Frischkorn, L. Hüwel und N.H. Nahler. Photodissoziation of $\mathrm{HBr}$ molecules and clusters: Anisotropy parameters, branching ratios, and kinetic energy distributions. J. Chem. Phys. 111 (1999) 2595.

[Bau00] R. Baumfalk, N.H. Nahler, U.Buck, M.Y. Niv und R.B. Gerber. Photodissociation of $\mathrm{HBr}$ adsorbed on the surface and embedded in large $\mathbf{A r}_{\mathrm{n}}$ clusters. J. Chem. Phys. $113(2000) 329$.

[Bau01a] R. Baumfalk, N.H. Nahler und U. Buck. Photodissociation and caging of $\mathrm{HBr}$ and HI molecules on the surface of large rare gas clusters. Faraday Discuss. 118 (2001) 247.

[Bau01b] R. Baumfalk, N.H. Nahler und U. Buck. Photodissociation of oriented HXel molecules in the gas phase. J. Chem. Phys. 114 (2001) 4755.

[Bau01c] R. Baumfalk, N.H. Nahler und U. Buck. Vibrational excitation and caging following the photodissociation of small $\mathrm{HBr}$ clusters in and on large Ar clusters. Phys. Chem. Chem. Phys. 3 (2001) 2372.

[Ber69] R. Bersohn und S.H. Lin. Orientation of Targets by Beam Excitation. Advan. Chem. Phys. 16 (1969) 67.

[Bet57] H.A. Bethe und E.E. Salpeter. Quantum Mechanics of One and Two-Electron Atoms (Springer, Berlin, 1957). 
[Bet81] D.S. Bethune. Dye Cell Design for High-Power Low-Divergence Excimer-Pumped Dye Lasers. Appl. Opt. 20 (1981) 1897.

[Bih02] Z. Bihary, G.M. Chaban und R.B. Gerber. Vibrational spectroscopy and matrix-site geometry of $\mathrm{HArF}, \mathrm{HKrF}, \mathrm{HXeCl}$ and $\mathrm{HXel}$ in rare-gas solids. J. Chem. Phys. 116 (2002) 5521.

[Blo92] P.A. Block, E.J. Bohac und R.E. Miller. Spectroscopy of Pendular States: The Use of Molecular Complexes in Achieving Orientation. Phys. Rev. Lett. 68 (1992) 1303.

[Boe92] U. Boesl, R. Weinkauf und E.W. Schlag. Reflectron Time-of-Flight Mass Spectrometry and Laser Excitation for the Analysis of Neutrals, lonized Molecules and Secondary Fragments. Int. J. Mass Spectrom. Ion Proc. 112 (1992) 121.

[Boo96] J.P. Booth, J. Derouard, M. Fadlallah, L. Cabaret und J. Pinard. Electric field measuremnts in discharges by Doppler-free two-photon laser Stark spetroscopy of atomic hydrogen. Opt. Comm. 132 (1996) 363.

[Bor98] I.B. Borges, G. Jalbert und C.E. Bielschowsky. Photon and electron-impact dissociation cross sections of HCl. J. Phys. B: At. Mol. Opt. Phys. 31 (1998) 3703.

[Bro74] M. Brown, J.T. Hougen, K.-P. Huber, J.W.C. Johns, I. Knopp, H. Lefebvre-Brion, A.J. Merer, D.A. Ramsey, J. Rostas und R.N. Zare. The Labeling of Parity Doublet Levels in Linear Molecules. J. Mol. Spec. 55 (1974) 500.

[Buc84] U. Buck und H. Meyer. Scattering Analysis of Cluster Beams: Formation and Fragmentation of Small Ar-Clusters. Phys. Rev. Lett. 52 (1984) 109.

[Buc96] U. Buck und R. Krohne. Cluster size determination from diffractive He atom scattering. J. Chem. Phys. 105 (1996) 5408.

[Buc02] U. Buck. Photodissociation of hydrogen halide molecules in different cluster environments. J. Phys. Chem. (2002). Eingereicht.

[Bus72] G.E. Busch und K.R. Wilson. Triatomic Photofragment Spectra. II. Angular Distributions from $\mathrm{NO}_{2}$ Photodissociation. J. Chem. Phys. 56 (1972) 3638.

[Cai01] L. Cai, J. Marango und B. Friedrich. Time-Dependent Alignment and Orientation of Molecules in Combined Electrostatic and Pulsed Nonresonant Laser Fields. Phys. Rev. Lett. 86 (2001) 775.

[Cha87] D.A. Chapman, K. Balasubramanian und S.H. Lin. A Theoretical Study of Spectroscopic Properties and Transition Moments of HBr. Chem. Phys. 118 (1987) 333.

[Cha01] A. Charvat, J. Aßmann und B. Abel. Real-Time Probing of Intramolecular Vibrational Energy Redistribution and Intermolecular Vibrational Energy Transfer of Selectively Excited $\mathrm{CH}_{2} \mathbf{I}_{2}$ Molecules in Solution. J. Phys. Chem. A 105 (2001) 5071. 
[Che02] B.-M. Cheng, C.-Y. Chung, M. Bahou, Y.-P. Lee und L.C. Lee. Quantitative spectral analysis of $\mathrm{HCl}$ and $\mathrm{DCl}$ in $\mathbf{1 2 0 - 2 2 0} \mathrm{nm}$ : Effects of singlet-triplet mixing. J. Chem. Phys. 117 (2002) 4293.

[Coh01] A. Cohen, M.Y. Niv und R.B. Gerber. Formation of novel rare-gas-containing molecules by molecular photodissociation in clusters. Faraday Discuss. 118 (2001) 269.

[Cox91] J.A. Coxon und P.G. Hajigeorgiu. Isotopic dependence of Born-Oppenheimer breakdown effects in diatomic hydrides: the $X^{1} \Sigma^{+}$states of $\mathrm{HI} / \mathrm{DI}$ and $\mathrm{HBr} / \mathrm{DBr}$. J. Mol. Spectrosc. 150 (1991) 1.

[Des94] S.R. Desai, C.S. Feigerle und J.C. Miller. Laser ionization mass spectrometry of homogenous and binary molecular clusters of nitric oxide. J. Chem. Phys. 101 (1994) 4526.

[Duh95] T. Duhoo und B. Pouilly. Analytical semiclassical calculation of photodissociation of the HCl molecule. J. Chem. Phys. 103 (1995) 182.

[Elo99] J. Eloranta, K. Vaskonen und H. Kunttu. Photogeneration of atomic hydrogen in rare gas matrices. J. Chem. Phys. 110 (1999) 7917.

[Fou91] O. Foucher, F. Aguillon und R. Campargue. Third harmonic generation of extreme ultraviolet radiation in a nitrogen continuous free jet zone of silence. J. Chem. Phys. 94 (1991) 4141.

[Fra34] J. Franck und E. Rabinowitsch. Some Remarks about Free Radicals and the Photochemistry of Solutions. Trans. Faraday Soc. 30 (1934) 120.

[Fri91] B. Friedrich und D. Herschbach. On the possibility of orienting rotationally cooled polar molecules in an electric field. Z. Phys. D-Atoms, Molecules and Clusters 18 (1991) 153.

[Fri95a] B. Friedrich und D.R. Herschbach. Alignment and Trapping of Molecules in Intense Laser Fields. Phys. Rev. Lett. 74 (1995) 4623.

[Fri95b] B. Friedrich und D.R. Herschbach. Polarization of Molecules Induced by Intense Nonresonant Laser Fields. J. Phys. Chem. 99 (1995) 15686.

[Fri97] C. Frischkorn. Photodissoziation von HBr-Molekülen und HBr-Clustern. Dissertation, Bericht 2/1998, MPI für Strömungsforschung, Göttingen (1997).

[Fri99a] B. Friedrich und D.R. Herschbach. Enhanced orientation of polar molecules by combined electrostatic and nonresonant induced dipole forces. J. Chem. Phys. 111 (1999) 6157.

[Fri9gb] B. Friedrich und D.R. Herschbach. Manipulating Molecules via Combined Static and Laser Fields. J. Phys. Chem. A 103 (1999) 10280. 
[Gar94] A. García-Vela, R. B. Gerber und U. Buck. Photolysis of $\mathbf{H C l}$ in the $\mathbf{A r}_{2}-\mathbf{H C l}$ and Ar-HCl Clusters: The Cluster Size Effect. J. Phys. Chem. 98 (1994) 3518.

[Gen98] D.J. Gendron und J.W. Hepburn. Dynamics of HI photodissoziation an the A band absorption via $\mathbf{H}$-atom doppler spectroscopy. J. Chem. Phys. 109 (1998) 7205.

[Ger93] I.H. Gersonde und H. Gabriel. Molecular dynamics of photodissociation in matrices including nonadiabatic processes. J. Chem. Phys. 98 (1993) 2094.

[Ger94a] R.B. Gerber, A.B. McCoy und A. García-Vela. Photochemical reactions in weakly bound clusters. Ann. Rev. Phys. Chem. 45 (1994) 275.

[Ger94b] I.H. Gersonde, S. Hennig und H. Gabriel. Nonadiabatic transitions in the photodissociation of HCl. J. Chem. Phys. 101 (1994) 9558.

[Gö96] K.H. Gödderz, N. Schwentner und M. Chergui. Cage exit probability versus excess energy in the photodissociation of matrix-isolated HCl. J. Chem. Phys. 105 (1996) 451.

[Gou85] T.E. Gough, M. Mengel, P.A. Rowntree und G. Scoles. Infrared spectroscopy at the surface of clusters: SF $_{6}$ on Ar. J. Chem. Phys. 83 (1985) 4958.

[Gre97] R.J. Green, J. Xie, R.N. Zare, A.A. Viggiano und R.A. Morris. Rate Constants and Products for the Reaction of $\mathrm{HBr}^{+}$with $\mathrm{HBr}$ and $\mathrm{DBr}$. Chem. Phys. Lett. 277 (1997) 1.

[Hab94] H. Haberland (Hg.). Clusters of Atoms and Molecules (Springer, Berlin, 1994).

[Hag81] Otto F. Hagena. Nucleation and Growth of Clusters in Expanding Nozzle Flows. Surf. Sci. 106 (1981) 101.

[Hag87] Otto F. Hagena. Condensation in Free Jets: Comparison of Rare Gases and Metals. Z. Phy. D 4 (1987) 291.

[Hag92] Otto F. Hagena. Cluster ion sources. Rev. Sci. Instrum. 63 (1992) 2374.

[Har86] I.A. Harris, K.A. Norman, R.V. Mulkern und J.A. Northby. Icosahedral structure of large charged argon clusters. Chem. Phys. Lett. 130 (1986) 316.

[Har88] A.L. Harris, J.K. Brown und C.B. Harris. The Nature of Simple Photodissociation Reactions in Liquids on Ulrafast Time Scales. Ann. Rev. Phys. Chem. 39 (1988) 341.

[Har94] M. Hartmann. Untersuchung unimolekularer Zerfälle im Molekularstrahl mittels Multiphotonenionisation. Diplomarbeit, Universität Göttingen (1994).

[Her91] G. Herzberg. Molecular Spectra and Molecular Structure: Spectra of Diatomic Molecules, Bd. 1 (Krieger, Malabar, 1991), 2. Aufl. 
[Hig98] J. Higgins, C. Callegari, J. Reho, F. Stienkemeier, W.E. Ernst, M. Gutowski und G. Scoles. Helium Cluster Isolation Spectroscopy of Alkali Dimers in the Triplet Manifold. jpca 102 (1998) 4952.

[Hil81] R. Hilbig und R. Wallenstein. Enhanced Production of Tunable VUV Radiation by Phase-Matched Frequency Tripling in Kypton and Xenon. IEEE Journal of Quantum Electronics 17 (1981) 1566.

[Hob94] M. Hobein. Photodissoziation und Photoionisation von Molekülclustern. Dissertation, Bericht 20/1994, MPI für Strömungsforschung, Göttingen (1994).

[Hui93] F. Huisken und M. Stemmler. Infrared spectroscopy of methanol clusters adsorbed on large $\operatorname{Ar}_{x}$ host clusters. J. Chem. Phys. 98 (1993) 7680.

[JM00] J.C. Juanes-Marcos und A. García-Vela. An energy-resolved study of the partial fragmentation dynamics of $\mathrm{Ar}-\mathrm{HCl}$ into $\mathrm{H}+\mathrm{Ar}-\mathrm{Cl}$ after ultraviolet photodissociation. J. Chem. Phys. 112 (2000) 4983.

[Joh81] P.M. Johnson und C.E. Otis. Molecular Multiphoton Spectroscopy with Ionization Detection. Ann. Rev. Phys. Chem. 32 (1981) 139.

[Joh99] M. Johansson, M. Hotokka, M. Pettersson und M. Räsänen. Quantum chemical potential energy surfaces for $\mathbf{H X e C l}$. Chem. Phys. 244 (1999) 25.

[Kak97] S. Kakar, O. Björneholm, J.O. Löfken, F. Federmann, A.V. Soldatov und T. Möller. Sizedependent core-level spectroscopy of free neutral clusters. Z. Phys. D 40 (1997) 84.

[Kar72] V.I. Karatev, B.A. Mamyrin und D.V. Shmikk. New method for focussing ion bunches in time-of-flight mass spectrometers. Sov. Phys. Tech. Phys. 16 (1972) 1177.

[Khr00] L. Khriachtchev, M. Pettersson, N. Runeberg, J. Lundell und M. Räsänen. A stable argon compound. Nature 406 (2000) 874.

[Kin92] T. Kinugawa und T. Arikawa. Three-dimensional Velocity Analysis Combining Ion Imaging with Doppler Spectroscopy: Application to Photodissociation of $\mathrm{HBr}$ at 243 nm. J. Phys. Chem. 96 (1992) 4801.

[Kra65] K.H. Kramer und R.B. Bernstein. Focusing and Orientation of Symmetric-Top Molecules with the Electric Six-Pole-Field. J. Chem. Phys. 42 (1965) 767.

[Kre99] C.J. Kreher, R.T. Carter und J.R. Huber. Photodissociation of OCIO and Ar/OCIO and $\mathrm{H}_{2} \mathrm{O} / \mathrm{OCIO}$ clusters studied by the resonance multiphoton ionization-time of flight method. J. Chem. Phys. 110 (1999) 3309. 
[Kro93] R. Krohne. He-Atom-Streuung zur Größenbestimmung und Schwingungsanregung großer Cluster. Dissertation, Bericht 18/1993, MPI für Strömungsforschung, Göttingen (1993).

[Kry97] A.I. Krylov und R.B. Gerber. Photodissociation Dynamics of $\mathbf{H C l}$ in solid Ar: Cage Exit, Nonadiabatic Transitions, and Recombination. J. Chem. Phys. 106 (1997) 6574.

[Kun94] H.M. Kunttu und J.A. Seetula. Photogeneration of ionic species in Ar, Kr and Xe matrices doped with $\mathbf{H C l}, \mathbf{H B r}$ and HI. Chem. Phys. 189 (1994) 273.

[Lam98] H.M. Lambert, P.-J. Dagdigian und M.H. Alexander. Spin-Orbit Branching in the Photofragmentation of $\mathbf{H C l}$ at Long Wavelength. J. Chem. Phys. 108 (1998) 4460.

[Lan98] S.R. Langford, P.M. Regan, A.J. Orr-Ewing und M.N.R. Ashfold. On the UV photodissociation dynamics of hydrogen iodide. Chem. Phys. 231 (1998) 245.

[Lar99] J.L. Larsen, H. Sakai, C.P. Salvan, I. Wendt-Larsen und H. Stapelfeldt. Aligning molecules with intense nonresonant laser fields. J. Chem. Phys. 111 (1999) 7774.

[Lat97] Z. Latajka und S. Scheiner. Structure, energetics and vibrational spectra of dimers, trimers, and tetramers of $\mathbf{H X}(\mathbf{X}=\mathbf{C l}, \mathbf{B r}, \mathbf{I})$. Chem. Phys. 216 (1997) 37.

[Law88] W. Lawrence, F. Okada und V.A. Apkarian. Experimental Studies on Photodissociation of HI in crystalline Xenon. Chem. Phys. Lett. 150 (1988) 339.

[Let87] V.S. Letokhov. Laser Photoionisation Spectroscopy (Academic Press, New York, 1987).

[Lev85] J.S. Levine (Hg.). Photochemistry of Atmospheres (Academic Press, New York, 1985).

[Lid96] David R. Lide, Hg. Handbook of chemistry and physics (CRC Press, 1996), 76 Aufl.

[Lig02] A. Lignell, L. Khriachtchev, M. Pettersson und M. Räsänen. Large blueshift of the $\mathrm{H}-\mathrm{Kr}$ stretching frequency of $\mathrm{HKrCl}$ upon complexation with $\mathrm{N}_{2}$. J. Chem. Phys. 117 (2002) 961.

[Lin84] S.H. Lin, Y. Fujimura, H.J. Neusser und E.W. Schlag. Multiphoton Spectroscopy of Molecules (Academic Press, New York, 1984).

[Lin02] J. J. Lin, Y. Chen, Y.Y. Lee, Y.T. Lee und X. Yang. Photodissociation dynamics

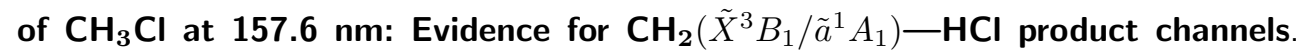
Chem. Phys. Lett. 361 (2002) 374.

[Liu93] Q. Liu, J.-K. Wang und A.H. Zewail. Femtosecond Dynamics of Dissociation and Recombination in Solvent Cages. Nature 364 (1993) 427.

[Liu99] K. Liu, A. Kolessov, J.W. Partin, I. Bezel und C. Wittig. Probing the $\mathrm{Cl}-\mathrm{HCl}$ complex via bond-specific photodissociation of $(\mathrm{HCl})_{2}$. Chem. Phys. Lett. 299 (1999) 374. 
[Liy95] R. Liyanage, Yung an Yang, S. Hashimoto, R.J. Gordon und R.W. Field. Electronic control of the spin-orbit branching ration in the photodissociation and predissociation of HCl. J. Chem. Phys. 103 (1995) 6811.

[Loh93] P. Lohbrandt. Erzeugung und Fragmentierung größenselektierter Cluster. Diplomarbeit, Universität Göttingen (1993).

[Loh01] P. Lohbrandt, R. Galonska, H.-J. Kim, M. Schmidt, C. Lauenstein und U. Buck. Electron Impact Fragmentation of Size Selected $\operatorname{Ar}_{n}(n=4$ to 9) Clusters. In R. Campargue, Hg., Atomic and Molecular Beams (Springer, 2001) (Seiten 623-636).

[Lor00] M. Lorenz, D. Kraus, M. Räsänen und E. Bondybey. Photodissociation of hydogen halides in rare gas matrices, and the effect of hydrogen bonding. J. Chem. Phys. $112(2000) 3803$.

[Lun00a] J. Lundell, G.M. Chaban und R.B. Gerber. Anharmonic Vibrational Spectroscopy Calculations for Novel Rare-Gas-Containing Compounds: $\mathrm{HXel}, \mathrm{HXeCl}, \mathrm{HXeBr}$ and HXeOH. J. Phys. Chem. A 104 (2000) 7944.

[Lun00b] J. Lundell, M. Pettersson, L. Khriachtchev, M. Räsänen, G.M. Chaban und R.B. Gerber. Infrared spectrum of HXel revisited: anharmonic vibrational calculations and matrix isolation experiments. Chem. Phys. Lett. 322 (2000) 389.

[Lun00c] J. Lundell, M. Pettersson und M. Räsänen. Computer experiments on xenoncontaining molecules. Computer and Chemistry 24 (2000) 325.

[Mah79] R. Mahon, T. J. Mcllrath, V. P. Myerscough und D. W. Koopman. Third-Harmonic Generation in Argon, Krypton and Xenon: Bandwidth Limitations in the Vicinity of Lyman- $\alpha$. IEEE Journal of Quantum Electronics 15 (1979) 444.

[Mar00] G. Maroulis. Is the dipole polarizability of hydrogen iodide accurately known? Chem. Phys. Lett. 318 (2000) 181.

[Mat90] Y. Matsumi, K. Tonokura, M. Kawasaki und T. Ibuki. Photodissociation of Hydrogen Chloride and Hydrogen Bromide. J. Chem. Phys. 93 (1990) 7981.

[Mat92] Y. Matsumi, P.K. Das und M. Kawasaki. Doppler spectroscopy of chlorine atoms generated from photodissociation of hydrogen chloride and methyl chloride at 157 and 193 nm. J. Chem. Phys. 97 (1992) 5261.

[McC93] A.B. McCoy, Y. Hurwitz und R.B. Gerber. Dynamics of Photoinduced Reactions in Hydrogen-Bonded Clusters: Classical Studies of the Photodissociation of $\mathbf{H C l}_{2}$. J. Phys. Chem. 97 (1993) 12516.

[Mil88] D.R. Miller. Free Jet Sources. In G. Scoles, Hg., Atomic and Molecular Beam Methods, Bd. 1 (Oxford, New York, 1988) (Seiten 14-53). 
[Moo52] C.E. Moore. Atomic Energy Levels, Bd. 1 - 3 (National Bureau of Standards, Washington - DC, USA, 1952). Circular 467.

[Mul37] R.S. Mulliken. Low Electronic States of Simple Heteropolar Diatomic Molecules: III. Hydrogen and Univalent Metal Halides. Phys. Rev. 51 (1937) 310.

[Mul40] R.S. Mulliken. Intensities in Molecular Electronic Spectra. X. Calculations on MixedHalogen, Hydogen Halide, Alkyl Halide, and Hydroxyl Spectra. J. Chem. Phys. 8 (1940) 382.

[Mül90] R. Müller. Massenanalyse neutraler Van-der-Waals Cluster mit einem Time-offlight Massenspektrometer. Diplomarbeit, Universität Hamburg (1990).

[Nah98] N. H. Nahler. Nachweis von Photodissoziationsprodukten durch Mehrphotonenionisation. Diplomarbeit, Universität Göttingen (1998).

[Nah02] N.H. Nahler, R. Baumfalk, U. Buck, Z. Bihary, R.B. Gerber und B. Friedrich. Photodissociation of oriented $\mathrm{HXel}$ molecules generated from $\mathrm{HI}-\mathrm{Xe}_{\mathrm{n}}$ clusters. J. Chem. Phys. in Vorbereitung (2002) xxx.

[Nee86] J. B. Nee, M. Suto und L. C. Lee. Quantitative Spectroscopy Study of $\mathbf{H B r}$ in the 105-235 nm Region. J. Chem. Phys. 85 (1986) 4919.

[Niv97] M.Y. Niv, A.I. Krylov und R.B. Gerber. Photodissociation, Electronic Relaxation and Recombination of $\mathrm{HCl}$ in $\mathrm{Ar}_{n}(\mathrm{HCl})$ Clusters: Nonadiabatic Molecular Dynamics Simulations. Faraday Discussions 108 (1997) 243.

[Niv99] M.Y. Niv, A.I. Krylov, R.B. Gerber und U. Buck. Photodissociation of $\mathbf{H C l}$ absorbed on the surface of an $\mathrm{Ar}_{12}$ cluster: Nonadiabatic molecular dynamics simulations. J. Chem. Phys. 110 (1999) 11047.

[OE97] A.J. Orr-Ewing, W.R. Simpson, T.P. Rakitzis, S.A. Kandel und R.N. Zare. ScatteringAngle Resolved Product Rotational Alignment for the Reaction of $\mathrm{Cl}$ with Vibrationally Excited Methane. J. Chem. Phys. 106 (1997) 5961.

[Pap91] J.M. Papanikolas, J.R. Gord, N.E. Levinger, D. Ray, V. Vorsa und W.C. Lineberger. Photodissociation and Geminate Recombination Dynamics of $\mathrm{I}_{2}^{-}$in Mass-Selected $\mathbf{I}_{2}^{-}\left(\mathrm{CO}_{2}\right)_{n}$ Cluster lons. J. Phys. Chem. 95 (1991) 8028.

[Pap93] J.M. Papanikolas, V. Vorsa, M.E. Nadal, P.J. Campagnola, H.K. Buchenau und W.C. Lineberger. $\mathrm{I}_{2}^{-}$photodissociation and recombination dynamics in size-selected $\mathrm{I}_{2}^{-}\left(\mathrm{C}_{2} \mathrm{O}\right)_{n}$ cluster ions. J. Chem. Phys. 99 (1993) 8733.

[Peo97] G. Peoux, M. Monnerville, T. Duhoo und B. Pouilly. Spin-Orbit Branching in the Photodissociation of HBr: Time-Independent, Time-Dependent and Semiclassical Calculations. J. Chem. Phys. 107 (1997) 70. 
[Pet95] M. Pettersson, J. Lundell und M. Räsänen. Neutral rare-gas containing charge-transfer molecules in solid matrices. I. $\mathrm{HXeCl}, \mathrm{HXeBr}, \mathrm{HXel}$, and $\mathrm{HKrCl}$ in $\mathrm{Kr}$ and $\mathrm{Xe}$. J. Chem. Phys. 102 (1995) 6423.

[Pet97] M. Pettersson, J. Nieminen, L. Khriachtchev und M. Räsänen. The mechanism of formation and infrared-induced decomposition of $\mathrm{HXel}$ in solid Xe. J. Chem. Phys. 107 (1997) 8423.

[Pet99] M. Pettersson, J. Lundell und M. Räsänen. New Rare-Gas-Containing Neutral Molecules. Eur. J. Inorg. Chem. 5 (1999) 729.

[Pet02a] M. Pettersson. Private Mitteilungen zu Dissoziationsenergien von $\mathbf{H K r F , ~} \mathbf{H K r C l}$ und $\mathrm{HXeBr}$ (2002).

[Pet02b] M. Pettersson, L. Khriachtchev, A. Lignell, M. Räsänen, Z. Bihary und R.B. Gerber. HKrF in solid krypton. J. Chem. Phys. 116 (2002) 2508.

[Pic01] C.A. Picconatto, H. Ni, A. Srivastava und J.J. Valentini. Quantum state distributions of $\mathbf{H C l}$ from the ultraviolet photodissociation of $\mathbf{H C l}$ dimer. J. Chem. Phys. 114 (2001) 7073.

[Pie97] R. von Pietrowski, M. Rutzen, K. von Haeften, S. Kakar und T. Möller. Fluorescence excitation spectroscopy of Xenon doped Neon clusters: size and site effects, and cluster melting. Z. Phys. D 40 (1997) 22.

[Póc91] I. Pócsik. Lognormal distribution as the natural statistics of cluster systems. Z. Phys.. D - Atoms, Molecules and Clusters 20 (1991) 395.

[Pou98] B. Pouilly und M. Monnerville. New investigation of the photodissociation of the $\mathrm{HBr}$ molecule: total cross-section, anisotropy parameter and dependence of the spin-orbit branching on the ground state vibrational level. Chem. Phys. 238 (1998) 437.

[Reg99a] P.M. Regan, D. Ascenzi, C. Clementi, M.N.R. Ashfold und A.J. Orr-Ewing. The UV photodissociation of $\mathrm{HI}$ revisited: REMPI measurements of $\mathrm{I}\left({ }^{2} \mathrm{P}\right)$ atom spin-orbit branching fractions. Chem. Phys. Lett. 315 (1999) 187.

[Reg99b] P.M. Regan, S.R. Langfold, A.J. Orr-Ewing und M.N.R. Ashfold. The ultraviolett photodissociation dynamics of hydrogen bromide. J. Chem. Phys. 110 (1999) 281.

[Reg00] P.M. Regan, D. Ascenzi, A. Brown, G.G. Balint-Kurti und A.J. Orr-Ewing. Ultraviolet photodissociation of $\mathrm{HCl}$ in selected rovibrational states: Experiment and theory. J. Chem. Phys. 112 (2000) 10259.

[Ros92] J.M. Rost, J.C. Griffin, B. Friedrich und D.R. Herschbach. Pendular States and Spectra of Oriented Linear Molecules. Phys. Rev. Lett. 68 (1992) 1299. 
[Row91] F.S. Rowland. Stratospheric Ozone Depletion. Ann. Rev. Phys. Chem. 42 (1991) 731.

[Roy02] R.J. Le Roy, G.T. Kraemer und S. Manzhos. 1 Potential, 2 Potentials, 3 Potentials 4: Untagling the UV Photodissociation Spectra of HI and DI. J. Chem. Phys. in press (2002).

[Sat01] Hiroyasu Sato. Photodissociation of Simple Molecules in the Gas Phase. Chem. Rev. 101 (2001) 2687.

[Sch82] R. Schmiedl, H. Dugan, W. Meier und K.H. Welge. Laser Doppler Spectroscopy of Atomic Hydrogen in the Photodissociation of HI. Z. Phys. A 304 (1982) 137.

[Sch87] J. Schroeder und J. Troe. Elemetary Reactions in the Gas-Liquid Transition Range. Ann. Rev. Phys. Chem. 38 (1987) 163.

[Sch93] R. Schinke. Photodissociation Dynamics (Cambridge University Press, Cambridge, 1993).

[Sch95] T. Schröder, R. Schinke, S. Liu, Z. Bacic und J.W. Moskowitz. Photodissoziation of HF in $\operatorname{Ar}_{n}$ HF $(n=1-14,54)$ Van der Waals Clusters: Effects of the Solvent Cluster Size on the Solute Fragmentation Dynamics. J. Chem. Phys. 103 (1995) 9228.

[Sch96] S. Schütte. Mehrphotonen- und Elektronenstoßprozesse in Clustern. Dissertation, Bericht 7/1997, MPI für Strömungsforschung, Göttingen (1996).

[Sch98] M. Schmidt, R. Kusche, B.v. Issendorf und H. Haberland. Irregular variations of the melting point of size-selected clusters. Nature 393 (1998) 238.

[Sei95] T. Seideman. Rotational excitation and molecular alignment in intense laser fields. J. Chem. Phys. 103 (1995) 7887.

[Sie97] J.G. Siebers. Private Mitteilungen über Minimumskonfigurationen von $\mathbf{H C l}_{n}$ (1997).

[Sla00] P. Slavíček, P. Žd'ánská, P. Jungwirth, R. Baumfalk und U. Buck. Size Effects on Photodissociation and Caging of Hydrogen Bromide Inside or on the Surface of Large Inert Clusters: From One to Three Icosahedral Layers. J. Phys. Chem. 104 (2000) 7793.

[Sla01] P. Slavíček, M. Roselová, P. Jungwirth und B. Schmidt. Preference of cluster isomers as a result of quantum delocalization: Potential energy surfaces and intermolecular vibrational states of $\mathrm{Ne} \cdots \mathrm{HBr}, \mathrm{Ne} \cdots \mathrm{HI}$, and $\mathrm{HI}(\mathrm{Ar})_{\mathrm{n}}(\mathbf{n}=\mathbf{1 - 6})$. J. Chem. Phys. 114 (2001) 1539.

[Sla02a] P. Slavíček und P. Jungwirth. Private Mitteilungen zur $\mathbf{H B r}-\mathrm{Ne}_{n}$ Wechselwirkung (2002)

[Sla02b] P. Slavičcek und P. Jungwirth. Private Mitteilungen zur Photodissoziation von $\mathbf{H B r}$ und HI Molekülen in Ar Clusterumgebungen (2002). 
[Sof87] T.P. Softley, W.E. Ernst, L.M. Taschiro und R.N. Zare. A general purpose XUV laser spectrometer: Some applications to $\mathbf{N}_{2}, \mathbf{O}_{2}$ amd $\mathbf{C O}_{2}$. Chem. Phys. 116 (1987) 299.

[Sta89] J. Stapelfeldt. CLULU: Ein neues Experiment für Fluoreszenzuntersuchungen an Edelgas-Clustern vom Dimer bis zum Mikrokristall. Dissertation, Universität Hamburg (1989).

[Ste01] C. Steinbach. Streuung und Spektroskopie von Wasserstoffbrückensystemen. Dissertation, Bericht 5/2001, MPI für Strömungsforschung, Göttingen (2001).

[Ste02] C. Steinbach. Private Mitteilungen zur Größenverteilung von Ar Clustern (2002).

[Sya96] J.A. Syage. Photofragment Imaging by Sections for Measuring State-Resolved Angle-Velocity Differential Cross Sections. J. Chem. Phys. 105 (1996) 1007.

[Tar93] E.I. Tarasova, A.M. Ratner, V.M. Stepanenko, I.Ya. Fugol, M. Chergui, R. Schriever und N. Schwentner. Photodissociation of water in rare gas matrices: Cage effekt and local heating of the lattice. J. Chem. Phys. 98 (1993) 7786.

[Ton92] K. Tonokura, Y. Matsumi, M. Kawasaki, S. Tasaki und R. Bersohn. Photodissociation of Hydrogen Chloride at 157 and 193 nm: Angular Distributions of Hydrogen Atoms and Fine-Structure Branching Ratios of Chlorine Atoms in the ${ }^{2} P_{j}$ Levels. J. Chem. Phys. 97 (1992) 8210.

[Vac99] H. Vach. Impurity dynamics in binary van der Waals clusters created by pick-up. J. Chem. Phys. 111 (1999) 3536.

[Vac00] H. Vach. Solvation effects in large binary van der Waals clusters with high doping rates. J. Chem. Phys. 113 (2000) 1097.

[Vac02] H. Vach. Private Mitteilungen zum Eindringen von $\mathrm{HBr}$ und $\mathrm{HI}$ in Ar Cluster (2002).

[Van83] G.N.A. Van Veen, K.A. Mohamed, T. Baller und A.E. De Vries. Photofragmentation of HI in the First Continuum. Chem. Phys. 80 (1983) 113.

[Vas99] K. Vaskonen, J. Eloranta, T. Kiljunen und H. Kunttu. Thermal mobility of atomic hydrogen in solid argon and krypron matrices. J. Chem. Phys. 110 (1999) 2122.

[Vor97] V. Vorsa, S. Nandi, P.J. Campagnola, M. Larson und W.C. Lineberger. Recombination Dynamics of Photodissociated $\mathrm{I}_{2}^{-}$in Size Selected Ar and $\mathrm{CO}_{2}$ Clusters. J. Chem. Phys. 106 (1997) 1402.

[Vot83] C. Votava, R. Ahlrichs und A. Geiger. The HCl-HCl Interaction: From Quantum Mechanical Calculation to Properties of the Liquid. J. Phys. Chem. 78 (1983) 6841. 
[Wan95] J.-K. Wang, Q. Liu und A.H. Zewail. Solvation Ultrafast Dynamics of Reactions. 9. Femtosecond Studies of Dissociation and Recombination of lodine in Argon Clusters. J. Phys. Chem. 99 (1995) 11309.

[Wil55] W.C. Wiley und I.H. McLaren. Time-of-Flight Mass Spectrometer with Improved Resolution. Rev. Sci. Instrum. 26 (1955) 1150.

[Wri94] S.A. Wright und J.D. McDonald. Photoionization of an Autoionizing Level of Atomic Oxygen. J. Chem. Phys. 101 (1994) 238.

[Xu88] Z. Xu, B. Koplitz und C. Wittig. Determining Reaction Pathways and Spin-Orbit Populations in the Photodissociation of $\mathrm{HBr}$ and $\mathrm{HI}$ Using Velocity-Aligned Doppler Spectroscopy. J. Phys. Chem. 92 (1988) 5518.

[Zar72] R.N. Zare. Photoejection Dynamics. Mol. Photochem. 4 (1972) 1.

[Zar88] R.N. Zare. Angular Momentum - Understanding Spatial Aspects in Chemistry and Physics (John Wiley \& Sons, New York, 1988).

[Žd'á99] P. Žd'ánská, B. Schmidt und P. Jungwirth. Photolysis of hydrogen chloride embedded in the first argon solvation shell: Rotational control and quantum dynamics of photofragments. J. Chem. Phys. 110 (1999) 6246.

[Žd'á00] P. Žd'ánská, P. Slavíček und P. Jungwirth. $\mathbf{H C l}$ photodissociation on argon clusters: Effects of sequential solvation and librational preexcitation. J. Chem. Phys. 112 (2000) 10761.

[Zew92] A.H. Zewail, M. Dantus, R.M. Bowman und A. Mokhtari. Femtochemistry: recent advances and extension to high pressures. J. Photochem. Photobiol. A: Chem. 62 (1992) 301.

[Zew93] A.H. Zewail. Femtochemistry. J. Phys. Chem. 97 (1993) 12427.

[Zha96] J. Zhang, C.W. Riehn, M. Dulligan und C. Wittig. An experimental study of HF photodissociation: Spin-orbit branching ratio and infrared alignment. J. Chem. Phys. 104 (1996) 7027.

[Zha97] J. Zhang, M. Dulligan und C. Wittig. Photodissociation of $\mathbf{H C l}$ at 193.3 nm: SpinOrbit Branching Ratio. J. Chem. Phys. 107 (1997) 1403. 



\section{Danksagung}

Die vorliegende Arbeit entstand am Max-Planck-Institut für Strömungsforschung und wurde gefördert im Rahmen des Sonderforschungsbereichs 357 „Molekulare Mechanismen unimolekularer Prozesse".

Mein besonderer Dank gilt Herrn Prof. Dr. U. Buck für die wissenschaftliche Betreuung und die zahlreichen Diskussionen während dieser Arbeit. Insbesondere möchte ich ihm hier auch für die Geduld in der experimentellen Phase und für die engagierte Interpretation der Ergebnisse danken. Vielen Dank auch für die beiden Forschungsaufenthalte an der University of California Irvine.

Auf die Unterstützung durch und die Zusammenarbeit in der Arbeitsgruppe konnte ich mich immer verlassen. Vielen Dank an Dr. Patrik Andersson, Dr. Jesko Brudermann, Dr. Michal Fárník und Dr. Christof Steinbach. Ganz besonders möchte ich mich hier bei Dr. Reinhard Baumfalk bedanken, sowohl für die schöne Zeit an der P6 als auch die unzähligen experimentellen und wissenschaftlichen Diskussionen über die gesamte Dauer meiner Arbeit.

Eine experimentelle Arbeit läßt sich ohne die Hilfe vieler sonst ungenannter nicht durchführen. Hier möchte ich stellvertretend für den Service und die Werkstätten des Instituts Rainer Krohn, Wolfgang Stieler und Wolf Keiderling danken.

Für die Fehlerreduzierung des Manuskripts geht ein herzlicher Dank an Christof, Michal, Katja, Carola und Iska.

Mein besonderer Dank gilt meinen Eltern und meiner Schwester, die mich über die ganze Zeit meines Studiums in vielerlei Weise unterstützt haben.

Abschließend danke ich meiner Freundin Iska ganz außerordentlich für die Hilfe und Liebe nicht nur in der Endphase meiner Arbeit. Danke für die vielen Aufmunterungen und dein unermüdliches Verständnis.

Vielen Dank 



\section{Lebenslauf}

\section{Persönliche Daten}

\section{Name}

geboren

Nationalität

Familienstand

\section{Schulbildung}

$06 / 1993$

\section{Studium}

10/1993 - 09/1995

Wintersemester 1995

$10 / 1995$ - 11/1998

$10 / 1996-06 / 1998$

Wintersemester 1998

seit $12 / 1998$

seit 4/1999

\section{Anstellungen}

$4 / 1997-11 / 1998$

seit $12 / 1998$
Nils Hendrik Nahler

15. April 1974 in Kassel

deutsch

unverheiratet

Erwerb der Allgemeinen Hochschulreife

an der König-Heinrich-Schule in Fritzlar/Hessen

Studium Physik/Diplom an der Universität Konstanz Vordiplomprüfungen

Studium Physik/Diplom an der Universität Göttingen

Diplomarbeit am Max-Planck-Institut

für Strömungsforschung in Göttingen mit dem Thema:

„Nachweis von Photodissoziationsprodukten

durch Mehrphotonenionisation"

Diplomprüfungen

Dissertation unter der Betreuung von Prof. Dr. U. Buck mit dem Thema „Photodissoziation von Halogenwasserstoffund orientierten Wasserstoff-Edelgas-Halogen-

Molekülen in Clusterumgebungen"

Aufbaustudiengang Physik an der Universität Göttingen

Studentische Hilfskraft am MPI für Strömungsforschung

Wissenschaftl. Angestellter am MPI für Strömungsforschung 